\title{
Translational approach for new therapeutic targets to prevent severe neonatal morbidities
}

Citation for published version (APA):

Cetinkaya, M. (2019). Translational approach for new therapeutic targets to prevent severe neonatal morbidities: from bench to bedside. [Doctoral Thesis, Maastricht University]. ProefschriftMaken Maastricht. https://doi.org/10.26481/dis.20190917mc

Document status and date:

Published: 01/01/2019

DOI:

10.26481/dis.20190917mc

Document Version:

Publisher's PDF, also known as Version of record

\section{Please check the document version of this publication:}

- A submitted manuscript is the version of the article upon submission and before peer-review. There can be important differences between the submitted version and the official published version of record.

People interested in the research are advised to contact the author for the final version of the publication, or visit the DOI to the publisher's website.

- The final author version and the galley proof are versions of the publication after peer review.

- The final published version features the final layout of the paper including the volume, issue and page numbers.

Link to publication

\footnotetext{
General rights rights.

- You may freely distribute the URL identifying the publication in the public portal. please follow below link for the End User Agreement:

www.umlib.nl/taverne-license

Take down policy

If you believe that this document breaches copyright please contact us at:

repository@maastrichtuniversity.nl

providing details and we will investigate your claim.
}

Copyright and moral rights for the publications made accessible in the public portal are retained by the authors and/or other copyright owners and it is a condition of accessing publications that users recognise and abide by the legal requirements associated with these

- Users may download and print one copy of any publication from the public portal for the purpose of private study or research.

- You may not further distribute the material or use it for any profit-making activity or commercial gain

If the publication is distributed under the terms of Article $25 \mathrm{fa}$ of the Dutch Copyright Act, indicated by the "Taverne" license above, 


\section{TRANSLATIONAL APPROACH}

FORNEW THERAPEUTIC TARGETS

TO PREVENT

SEVERE NEONATAL MORBIDITIES :

\section{- FROM BENCH TO BEDSIDE}

\section{MERIH CETINKAYA}

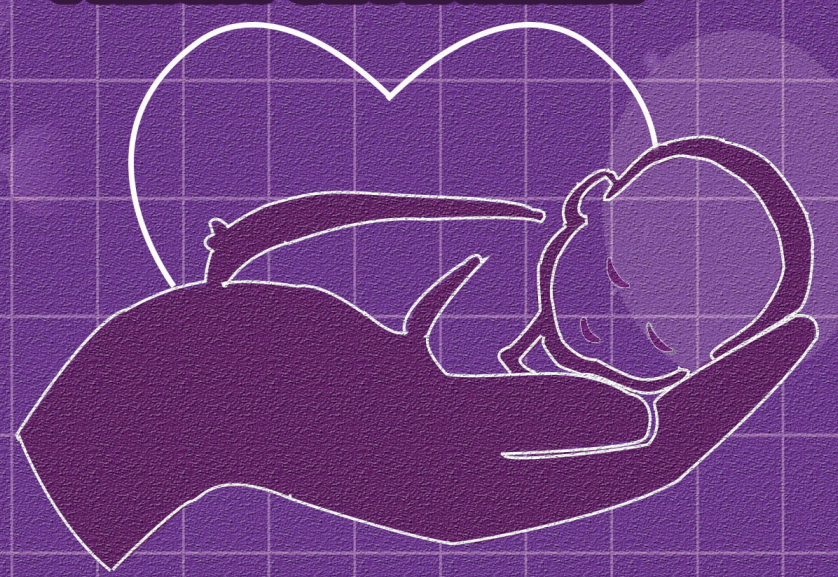




\section{Translational Approach for New Therapeutic Targets to Prevent Severe Neonatal Morbidities:}

From Bench to Bedside 
(C) copyright Merih Cetinkaya, Maastricht 2019

Printing: ProefschriftMaken || www.proefschriftmaken.nl

ISBN 9789463804844

All rights reserved. No part of this publication may be reproduced, stored in a retrieval system or transmitted, in any form or by any means, electronic, mechanical, photocopying, recording or otherwise, without prior permission of the author or the copyright-owning journals for previous published chapters. 


\title{
Translational Approach for New Therapeutic Targets to Prevent Severe Neonatal Morbidities: From Bench to Bedside
}

\author{
DISSERTATION \\ to obtain the degree of Doctor at the Maastricht University, \\ on the authority of the Rector Magnificus Prof. dr. Rianne M. Letschert, \\ in accordance with the decision of the Board of Deans, \\ to be defended in public \\ on Tuesday September 17, 2019 at 14:00 hours \\ by \\ Merih Cetinkaya, \\ born September 16, 1975 \\ Diyarbakır, Turkey
}




\section{Supervisors}

Prof. dr. B.W.W. Kramer

Prof. dr. T. Delhaas

\section{Co-supervisor}

Dr. D. Gavilanes

\section{Assessment Committee}

Prof. dr. L.J.I. Zimmermann (chairman)

Dr. P. Andriessen (Maximá Medisch Centrum, Veldhoven, The Netherlands)

Prof. dr. T.W. Orlikowsky (University Children's Hospital, Aachen, Germany)

Prof. dr. M.E.A. Spaanderman

Prof. dr. Y. Temel 


\section{CONTENTS}

\section{Chapter 1}

Introduction

\section{Chapter 2}

Protective effects of valproic acid, a histone deacetylase inhibitor, against hyperoxic lung injury in a neonatal rat model

\section{Chapter 3}

Cytidine 5'-diphosphocholine ameliorates hyperoxic lung injury in a neonatal rat model

\section{Chapter 4}

CDP-choline reduces severity of intestinal injury in a neonatal rat model of necrotizing enterocolitis

\section{Chapter 5}

Evaluation of melatonin and prostaglandin E1 combination on necrotizing enterocolitis model in neonatal rats

\section{Chapter 6}

Neuroprotective effects of uridine in a rat model of neonatal hypoxic-ischemic encephalopathy

\section{Chapter 7}

Possible neuroprotective effects of magnesium sulphate and melatonin as both preand post-treatment in a neonatal hypoxic-ischemic rat model

\section{Chapter 8}

Neuroprotective effects of melatonin administered alone or in combination with topiramate in neonatal hypoxic-ischemic rat model

\section{Chapter 9}

Discussion and conclusions

\section{Chapter 10}

Valorization

\section{Chapter 11}

Curriculum vitae 

Chapter

Introduction, Aim and Outline 



\section{Need for new therapies for severe neonatal morbidities}

Most of childhood deaths occur during the neonatal period, which extends from birth to the first 28 days of life (Pathirana et al., 2016). The 2.9 million annual neonatal deaths worldwide have been reported to be associated with prematurity complications, intrapartum related brain injury and infections (Lawn et al. 2014). As all these morbidities have high fatality rates, preventive measures are therefore needed either to reduce the development or to treat and prevent their progression into more severe forms (Lawn et al. 2014).

\section{Causes of preterm birth}

Preterm birth, defined by the World Health Organization as delivery before 37 weeks of gestation are completed (WHO, 1977), occurs in 1 of 10 pregnancies resulting in approximately 15 million premature deliveries worldwide (Blencowe et al., 2012). Twothirds of preterm birth occur after spontaneous onset of preterm labor. These cases have been mostly associated with several conditions such as preterm premature rupture of membranes (PPROM), intrauterine infection/inflammation, cervical insufficiency, uterine anomalies and distension (Romero et al., 2006). Morbidity and mortality after preterm birth are inversely associated with gestational age and birth weight. Preterm birth is not only associated with higher mortality rates, but also with increased morbidity severity, neurodevelopmental impairment, familial effects and significant costs (Frey et al., 2016). Preterm infants are also vulnerable to both short and long term complications including respiratory distress syndrome (RDS), chronic lung disease, injury to intestines, infections associated with compromised immune system, cardiovascular disorders, hearing and vision problems, and neurological impairment. All these preterm birth-related complications occur due to the immaturity of all organ systems; they are not yet prepared for extrauterine life (Bohlin, 2016). This thesis deals with three of the most common complications of preterm birth that will be introduced in the following sections.

\section{Complications of preterm birth}

Among the many complications after preterm birth that may have a long term impact on health, growth and development in preterm infants (Carter and Msell, 2017), bronchopulmonary dysplasia (BPD) is the most common complication/adverse outcome. BPD can be defined as a form of chronic lung disease as a result of impaired lung development. BPD was first described in 1967 (Northway et al., 1967). They reported this entity in babies of 32 weeks of gestation that had respiratory treatment for hyaline membrane disease. Because treatment options for preterm babies improved considerably in the past 50 years, BPD nowadays also occurs in extremely preterm infants (around 26 weeks of gestation) who are born during the late canalicular/early saccular stage of lung development. This form of BPD is associated with disrupted lung 
development (Jobe and Bancalari, 2001). Currently, BPD is diagnosed as the need for assisted ventilation and/or supplemental oxygen at 36' weeks postmenstrual age (Jobe and Bancalari, 2001). BPD has been diagnosed to develop in approximately $50 \%$ of extremely low birth weight (ELBW) preterm infants (Stoll et al., 2010). It is associated with higher mortality rates, and infants with BPD are prone to both respiratory and cardiovascular impairment with pulmonary hypertension, growth failure and neurodevelopmental delay (Carraro et al., 2013; Jensen et al., 2015; McEvoy et al., 2014; Stoll et al.,2010). As there is an improved survival for ELBW infants due to advances in perinatal and neonatal care, the incidence of BPD has not changed in recent years (Stoll et al, 2015). The etiology of BPD is multifactorial and includes mainly prematurity, antenatal/ postnatal infection and inflammation, exposure to mechanical ventilation and oxygen toxicity, all of which were suggested to contribute to impaired alveolar development and associated abnormal vascular growth (Bhandari and Bhandari, 2009). In recent years, both genetic and epigenetic mechanisms were reported to be associated with BPD development (Merritt et al., 2009). As BPD has a multifactorial pathogenesis, no definitive treatment strategy has been shown to be effective to reduce BPD incidence (Poets and Lorenz, 2018). Treatment modalities including antenatal steroids, surfactant, gentle ventilation strategies, targeted oxygen saturation goals, and optimization of nutrition just led to a modest improvement in BPD outcomes. Therefore, strategies aiming to prevent the development of BPD seems to be the key issue nowadays (Gien and Kinsella, 2011; McEvoy et al., 2014). In a recent review, postnatal steroids, caffeine, azithromycin and vitamin $A$ were reported as the only pharmacological therapies for prevention of BPD in preterm infants (Jensen et al., 2015). The authors also suggested dexamethasone therapy as an effective option for prevention of BPD which bears however the risk to induce adverse neurodevelopmental outcome (Jensen et al., 2015). Therefore, new therapies are needed to prevent development of BPD.

Necrotizing enterocolitis (NEC), defined as a multifactorial syndrome of acute intestinal ischemic necrosis, represents the most common neonatal gastrointestinal emergency, especially in preterm infants (Morgan et al., 2011). Prematurity is the most important risk factor and an inverse relationship was reported between gestational age and NEC development. It is less common in late preterm and term infants. Prematurity-associated intestinal immaturity, aggressive feeding strategies, and inflammatory response to interactions between intestinal epithelium and luminal microorganisms were suggested as the key contributors for NEC development (Sharma and Hudak, 2013). Intestinal and systemic perturbations commonly constitute the clinical presentation of NEC. Early systemic signs may be indistinguishable from neonatal sepsis (Cetinkaya et al., 2011). NEC may also progress to multiorgan system dysfunction and shock just within hours in a fulminant manner (Sharma and Hudak, 2013). Increased gastric residuals, abdominal distension, and emesis represent the most common initial clinical signs of NEC. Systemic signs usually include lethargy, hypotension, episodes of apnea/bradycardia, temperature 
instability, tachycardia and worsening respiratory status (Neu and Walker, 2011). In addition to clinical and laboratory findings, radiographic features including pneumatosis intestinalis and portal venous gas constitute the mainstay tools for diagnosis of NEC (Kasivajjula and Maheshwari, 2014). As no single treatment option has been shown to be effective after NEC diagnosis, preventive strategies focusing on the pathogenesis of NEC have gained importance in recent years. Preventive strategies comprise antenatal steroids, breast milk, microbiome interventions and standardized feeding regimens (Kasivajjula and Maheshwari, 2014).

Global hypoxia-ischemia is a birth-related complication that occurs in both preterm and term babies. Though global hypoxia-ischemia affects the whole body, organs like brain, kidney and the heart are most vulnerable. Brain injury inflicted by hypoxia-ischemia, called hypoxic- ischemic encephalopathy (HIE), is an important neonatal morbidity that, contrary to many other birth-related complications, mainly affects full term infants (Kurinczuk et al., 2011). The incidence of HIE ranges from 1 to 8 per 1000 live births in developed countries and is suggested to be higher in the developing countries (DouglasEscobar and Weiss, 2015). Hypoxic-ischemic insult during prenatal, intrapartum or postnatal period prevents adequate blood flow to the infant's brain. This impaired cerebral blood flow and oxygen delivery to the brain results in primary and secondary energy failure that triggers several pathways as an evolving process leading to cell death (Davidson et al., 2015; Johnston et al., 2011; Kimberly and Brandon, 2011). Some 40-60\% of affected infants die by 2 years of age or have severe disabilities such as cerebral palsy, mental retardation and epilepsy (Juul and Ferriero, 2014). Recent clinical evidence suggests that therapeutic hypothermia-induced reduced metabolic activity significantly improves survival and disability in term infants with mild to moderate HIE (Jacobs et al., 2013). As the brain damage occurs via multiple pathways and repair occurs over a period of days, combination therapies acting on the different pathways at different time points including excitotoxicity, inflammation and apoptosis, thereby providing more long-lasting neuroprotection, were suggested to be more effective for treatment of HIE (Jacobs et al., 2013; Juul and Ferriero, 2014). As therapeutic hypothermia is the standard effective treatment, this purpose can be achieved by adding a neuroprotective agent to hypothermia or by any combination of two different neuroprotective therapies. 


\section{Aim and outline of the thesis}

Before outlined approaches and ideas to improve the neonatal outcome can be applied in babies, solid supporting experimental data and understanding are needed. Translational models of neonatal morbidities should therefore be used to identify the possible underlying mechanisms and evaluate the effect of a therapy. Findings from these studies can also be used to establish a scientific interchange from experimental point to clinical usage in humans (Ward et al., 2006). The objective of this thesis was therefore to perform new experimental studies in rat models on a) hyperoxic lung injury, b) necrotizing enterocolitis, and c) hypoxic-ischemic brain injury in order to understand molecular and cellular mechanisms of these diseases and to investigate their therapeutic potentials. The therapeutic agents in these studies were selected in terms of their potential utility in neonates. Therefore, we evaluated the efficacy of several novel agents for prevention and treatment of important morbidities with multifactorial pathogenesis and limited definite treatment options.

\section{General Outline}

In Chapter 2, we tested the hypothesis that epigenetic mechanisms including histone acetylation-deacetylation process might play an important role in development of BPD. To this end, we used a neonatal rat model of hyperoxic lung injury in which we evaluated the efficacy of valproic acid, a histone-deacetylase inhibitor, for prevention of BPD.

Since surfactant deficiency was suggested to play a role in evolving BPD in infants, we tested in Chapter 3 the hypothesis that exogenous administration of CDP-choline, an endogenous intermediate in the synthesis of the major surfactant component phosphatidylcholine, is protective against hyperoxic lung injury in a neonatal rat model. In addition to the replacement of major surfactant component, the anti-inflammatory effects of this intermediate was investigated for the first time in an experimental setting.

In Chapter 4, we tested the hypothesis that major membrane phospholipid supplementation might ameliorate intestinal injury as the integrity of gut phospholipid barrier may confer benefit against intestinal tissue damage. Since intestinal membrane disruption, including the loss of membrane integrity and intestinal surfactant develops in the setting of NEC (Claud, 2009), we evaluated the effect of CDP-choline treatment in a neonatal NEC rat model.

Since NEC has a multifactorial pathogenesis including inflammation and necrosis, we tested in Chapter 5 the hypothesis that a combination therapy of anti-inflammatory and cytoprotective agents leads to prevention of NEC in a neonatal rat model of NEC.

Because epigenetic mechanisms are involved in the pathogenesis of $\mathrm{HIE}$, we investigated in Chapter 6 the neuroprotective effect of exogenously-administered uridine, as well as 
its possible mechanism of action with regard to modulating histone acetylation/deacetylation in a neonatal rat model of HIE. Uridine is the principal circulating pyrimidine nucleoside and a precursor for membrane phospholipid synthesis.

In Chapters 7 and 8, we tested in a neonatal rat model of HIE the hypothesis that the combination of agents acting at different stages of $\mathrm{HI}$ brain damage might provide superior neuroprotection compared to single use of each agent.

The thesis concludes with a General Discussion and Summary in Chapter 9. In this part, the results are put into broader perspective and the potential benefits of our studies in Neonatal Intensive Care Unit (NICU) practice and subsequent clinical studies are discussed. 


\section{References}

1. Bhandari, A., and Bhandari, V. (2009). Pitfalls, problems and progress in bronchopulmonary dysplasia. Pediatrics 123: 1562-1573.

2. Blencowe, H., Cousens, S., Oestergaard, M.Z., Chou, D., Moller, A.B., Narwal, R., Adler, A., Vera Garcia, C., Rohde, S., Say, L., and Lawn, J.E. (2012). National, regional, and worldwide estimates of preterm birth rates in the year 2010 with time trends since 1990 for selected countries: a systematic analysis and implications. Lancet 379: 2162-2172.

3. Bohlin, K. (2016). Cell-based strategies to reconstitute vital functions in preterm infants with organ failure. Best practice \&research. Clinical obstetrics \& gynaecology 31:99-111.

4. Carraro, S., Flippone, M., Da Dalt, L., Ferraro, V., Maretti, M., Bressan, S., El Mazloum, D., and Baraldi, E. (2013). Bronchopulmonary dysplasia: the earliest perhaps the longest lasting obstructive lung disease in humans. Early human development 89: S3-S5.

5. Carter, F.A., and Msell, M.E. (2017). Health disparities and child development after prematurity. Pediatric annals 46, e360-e364.

6. Cetinkaya, M., Ozkan, H., Koksal, N., Akacı, O., and Ozgur, T. (2011). Comparison of the efficacy of serum amyloid A, C-reactive protein, and procalcitonin in the diagnosis and follow-up of necrotizing enterocolitis in premature infants. Journal of pediatric surgery 46: 1482-1489.

7. Claud, E.C. (2009). Neonatal necrotizing enterocolitis-inflammation and intestinal immaturity. Antiinflammatory \& antiallergy agents in medicine chemistry 8: 248-259.

8. Davidson, J.O., Wassink, G., von den Heuij, L.G., Bennet, L., and Gunn A.J. (2015). Therapeutic hypothermia for neonatal hypoxic-ischemic encephalopathy-where to from here? Frontiers in neurology 6:198.

9. Douglas-Escobar, M., and Weiss, M.D. (2015). Hypoxic-ischemic encephalopathy: a review for the clinician. JAMA pediatrics 169: 397-403.

10. Frey, H.A., and Klebanoff, M.A. (2016). The epidemiology, etiology and costs of preterm birth. Seminars in fetal and neonatal medicine 21: 68-73.

11. Gien, J., and Kinsella, J.P. (2011). Pathogenesis and treatment of bronchopulmonary dysplasia. Current opinion in pediatrics 23: 305-313.

12. Jacobs, S.E., Berg, M., Hunt, R., Tarnow-Mordi, W.O., Inder, T.E., and Davis P.G. (2013). Cooling for newborns with hypoxic ischemic encephalopathy. Cochrane database syst rev 1: CD003311.

13. Jensen, E.A., Foglia, E.E., and Schmidt, B. (2015). Evidence-based pharmacological therapies for prevention of bronchopulmonary dysplasia. Clinics in perinatology 42: 775-779.

14. Jobe, A.H., and Bancalari, E. (2001). Bronchopulmonary dysplasia. American journal of respiratory and critical care medicine 163: 1723-1729.

15. Johnston, M.V., Fatemi, A., Wilson, M.A., and Northington, F. (2011). Treatment advances in neonatal neuroprotection and neurointensive care. Lancet neurology 10: 372-382.

16. Juul, S.E., and Ferriero, D.M. (2014). Pharmacological neuroprotective strategies in neonatal brain injury. Clinics in perinatology 41: 119-131.

17. Kasivajjula, H., and Maheshwari, A. (2014). Pathophysiology and current management of necrotizing enterocolitis. Indian journal of pediatrics 81: 489-497.

18. Kimberly, A.A., and Brandon, D.H. (2011). Hypoxic ischemic encephalopathy: pathophysiology and experimental therapies. Newborn and infant nursing reviews 11: 125-133.

19. Kurinczuk, J.J., White-Koning, M., and Badawi, N. (2011). Epidemiology of neonatal encephalopathy and hypoxic-ischemic encephalopathy. Early human development 86: 329-338.

20. Lawn, J.E., Blencowe, H., Oza, S., You, D., Lee, A.C., Waiswa, P., Lalli, M., Bhutta, Z., Barros, A.J., Christian, P., Mathers, C., Cousens, S.N., and Lancet Every Newborn Study Group. (2014). Every newborn: progress, priorities and potential beyond survival. Lancet 384: 189-205.

21. McEvoy, C.T., Jain, L., Schmidt, B., Abman, S., Bancalari, E., and Aschner, J.L.I. (2014) Bronchopulmonary dysplasia: NHLBI workshop on the primary prevention of chronic lung diseases. Annals of the american thoracic society 11 Suppl 3: S146-S153. 
22. Merritt, T.A., Deming, T.T., and Boynton, B.R. (2009). The new bronchopulmonary dysplasia: challenges and commentary. Seminars in Fetal Neonatal Medicine 14: 345-357.

23. Morgan, J.A., Young, L., and McGuire, W. (2011). Pathogenesis and prevention of necrotizing enterocolitis. Current opinion in infectious disease 24: 183-189.

24. Neu, J., and Walker, W.A. (2011). Necrotizing enterocolitis. New england journal of medicine 364: 255-264.

25. Northway, W.H. Jr., Rosan, R.C., and Porter, D.Y. (1967). Pulmonary disease following respiratory treatment of hyaline-membrane disease. Bronchopulmonary dysplasia. New england journal of medicine 276, 357368.

26. Pathirana, J., Munoz, F.M., Abbing-Karahagopian, V., Bhat, N., Harris, T., Kapoor, A., Keene, D.L., Mangili, A., Padula, M.A., Pande, S.L., Pool, V., Pourmalek, F., Varricchio F., Kochar, S., Cutland, C.L., and Brighton Collaboration Neonatal Death Working Group. (2016). Neonatal death: case definition \& guidelines for data collection, analysis, and presentation of immunization safety data. Vaccine 34: 6027-6037.

27. Poets, C.F., and Lorenz, L. (2018). Prevention of bronchopulmonary dysplasia in extremely low gestational age neonates: current evidence. Archieves of disease in childhood. Fetal and neonatal edition 103: F285F291.

28. Romero, R., Espinazo, J., Kusanovic, J.P., Gotsch, F., Hassan, S., Erez, O., Chaiworapongsa, T., and Mazor, P. (2006). The preterm parturition syndrome. BJOG: an international journal of obstetrics and gynecology 113 Suppl 3, 17-42.

29. Sharma, R., and Hudak, M.L. (2013). A clinical perspective of necrotizing enterocolitis. Past, present and future. Clinics in perinatology 40:27-51.

30. Stoll, B.J., Hansen, N.I., Bell, E.F., Shankaran, S., Laptook, A.R., Walsh, M.C., Hale, E.C., Newman, N.S., Schibler, K., Carlo, W.A., Kennedy, K.A., Poindexter, B.B., Finer, N.N., Ehrenkranz, R.A., Duara, S., Sánchez, P.J., O'Shea, T.M., Goldberg, R.N., Van Meurs, K.P., Faix, R.G., Phelps, D.L., Frantz, I.D 3rd., Watterberg, K.L., Saha, S., Das, A., Higgins, R.D., and Eunice Kennedy Shriver National Institute of Child Health and Human Development Neonatal Research Network. (2010). Neonatal outcomes of extremely preterm infants from the NIHCD Neonatal Research Network. Pediatrics 126, 443-456.

31. Stoll, B.J., Hansen, N.I., Bell, E.F., Walsh, M.C., Carlo, W.A., Shankaran, S., Laptook, A.R., Sánchez, P.J., Van Meurs, K.P., Wyckoff, M., Das, A., Hale, E.C., Ball, M.B., Newman, N.S., Schibler, K., Poindexter, B.B., Kennedy, K.A., Cotten, C.M., Watterberg, K.L., D’Angio, C.T., Demauro S.B., Truog, W.E., Devaskar, U., Higgins, R.D., and Eunice Kennedy Shriver National Institute of Child Health and Human Development Neonatal Research Network. (2015). Trends in care practices, morbidity and mortality of extremely preterm neonates, 1993-2012. JAMA 314: 1039-1051

32. Ward, R.M., Lane, R.H., and Albertine, K.H. (2006). Basic and translational research in neonatal pharmacology. Journal of perinatology 26: S8-S12.

33. WHO. (1977). WHO: recommended definitions, terminology and format for statistical tables related to the perinatal period and use of a new certificate for cause of perinatal deaths. Modifications recommended by FIGO as amended October 14, 1976. Acta obstetricia et gynecologica Scandinavica 56, 247-253. 



\title{
Chapter
}

\author{
Protective effects of valproic acid, a histone \\ deacetylase inhibitor, against hyperoxic lung \\ injury in a neonatal rat model
}

Published as: Cetinkaya M, Cansev M, Cekmez F, Tayman C, Canpolat FE, Kafa IM, Yaylagul EO, Kramer BW, Sarıcı SU. Protective effects of valproic acid, a histone deacetylase inhibitor, against hyperoxic lung injury in a neonatal rat model.

PLoS One 2015; 10(5): e0126028. 


\section{Abstract}

\section{Objective}

Histone acetylation and deacetylation may play a role in the pathogenesis of inflammatory lung diseases. We evaluated the preventive effect of valproic acid (VPA), a histone deacetylase (HDAC) inhibitor, on neonatal hyperoxic lung injury.

\section{Methods}

Forty newborn rat pups were randomized in normoxia, normoxia+VPA, hyperoxia and hyperoxia+VPA groups. Pups in the normoxia and normoxia+VPA groups were kept in room air and received daily saline and VPA (30 mg/ $\mathrm{kg}$ ) injections, respectively, while those in hyperoxia and hyperoxia+VPA groups were exposed to $95 \% \mathrm{O}_{2}$ and received daily saline and VPA (30 mg/kg) injections for 10 days, respectively. Growth, histopathological, biochemical and molecular biological indicators of lung injury, apoptosis, inflammation, fibrosis and histone acetylation were evaluated.

\section{Results}

VPA treatment during hyperoxia significantly improved weight gain, histopathologic grade, radial alveolar count and lamellar body membrane protein expression, while it decreased number of TUNEL (+) cells and active Caspase-3 expression. Expressions of TGF 33 and phospho-SMAD2 proteins and levels of tissue proinflammatory cytokines as well as lipid peroxidation biomarkers were reduced, while anti-oxidative enzyme activities were enhanced by VPA treatment. VPA administration also reduced HDAC activity while increasing acetylated $\mathrm{H} 3$ and $\mathrm{H} 4$ protein expressions.

\section{Conclusions}

The present study shows for the first time that VPA treatment ameliorates lung damage in a neonatal rat model of hyperoxic lung injury. The preventive effect of VPA involves HDAC inhibition. 


\section{Introduction}

Bronchopulmonary dysplasia (BPD) is a chronic lung disease developing especially in premature infants with significant morbidity and mortality [1]. The pathogenesis of BPD is multifactorial resulting in chronic inflammation of the airways that results in a simplified lung structure [2]. To date, despite antenatal steroids, vitamin A, and caffeine being suggested as beneficial agents for BPD prevention, BPD is still the most common adverse outcome after preterm birth and the use of postnatal steroids as potent antiinflammatory drugs is still under study $[1,3,4]$. Recent evidence suggests a net benefit of postnatal glucocorticoid therapy when administered shortly after the first week of life to premature infants with early and persistent pulmonary dysfunction [5]. Similarly, in a recent Cochrane review, late steroid therapy after 7 days of life for chronic lung disease was suggested to reduce neonatal mortality without significantly increasing the risk of adverse long-term neurodevelopmental outcomes [6]. Therefore, it is necessary to develop new strategies for the prevention of BPD.

Chronic inflammation can be maintained by different triggers which promote transcription of pro-inflammatory cytokines. Transcription factors target gene transcription after activation and recruit transcriptional co-activators and chromatinremodeling enzymes that allows subsequent inflammatory gene expression [7]. The transcription of genes is mainly made possible by rearrangement of the chromatin structure by histone methylation and deacetylation, which are all epigenetic modification [8]. Epigenetic regulation of inflammation has therefore several components. Changes in histone acetylation are involved in induction of pro-inflammatory genes in human lung cells. Histone acetyltransferases (HATs) are expressed and activated in an abnormal fashion in inflammatory diseases and, histone deacetylase (HDAC) inhibitors show antiinflammatory effects [9]. Differentially-regulated chromatin remodeling pathways were reported in umbilical cord samples of infants who developed BPD by altering HDAC ratio, resulting in histone hypoacetylation. This suggests the use of HDAC inhibitors for the prevention of BPD development [10]. However, current therapies of BPD do not modulate the epigenetic compound of chronic inflammation.

A candidate drug for this purpose is Valproic acid (VPA), an HDAC inhibitor that is clinically used as an anti-epileptic and mood-stabilizing agent. VPA is an HDAC inhibitor of class I, as well as class II histone deacetylases [11] which results in anti-inflammatory properties by reducing transcription of pro-inflammatory cytokines $[12,13]$. This effect was shown in a lipopolysaccharide (LPS)-induced septic shock model where VPA attenuated multiple organ dysfunctions by ameliorating histopathological lung injury, preventing pulmonary inflammation by reversing the reduction in histone $\mathrm{H} 3$ acetylation in lung tissue [14].

However, to the best of our knowledge, no experimental study has investigated VPA's effectiveness in neonatal hyperoxic lung injury. Therefore, the aim of this study was to evaluate the possible preventive effect of VPA in a neonatal rat model of hyperoxic lung 
injury and the involvement of HDAC inhibition in VPA's effect. We used a rat model in which neonatal exposure to hyperoxia induced lung simplification resembling the lung structure of patients with BPD.

\section{Material and Methods}

\section{Animals and experimental design}

The study was approved by Experimental Animal Ethics Committee of Gulhane Military Medical Academy (Ankara, Turkey, Permit number: 2011-6) and the experiments conformed to the National Institutes of Health Guide for the Care and Use of Laboratory Animals (NIH Publications No. 80-23) revised 1996 and EC Directive 86/609/EEC.

Sprague-Dawley rats with dated pregnancies were housed in individual cages with free access to water and food. Pups born with spontaneous delivery to Sprague-Dawley pregnant rats were pooled, randomized, and delivered back to nursing dams. The offsprings from one dam were randomly introduced into all four study groups. A total of 40 pups born to 4 dams were divided into 4 groups from each dam as follows: normoxia group (subjected to room air containing $21 \% \mathrm{O}_{2}$ and received saline), normoxia+VPA group (subjected to room air containing $21 \% \mathrm{O}_{2}$ and received VPA), hyperoxia group (subjected to $95 \% \mathrm{O}_{2}$ and received saline), and hyperoxia+VPA group (subjected to $95 \%$ $\mathrm{O}_{2}$ and received VPA).

Experiments began immediately after birth and continued throughout P10 as described previously [15]. Nursing dams were rotated between hyperoxia and room airexposed pups every $24 \mathrm{~h}$ to prevent oxygen toxicity. In pups subjected to hyperoxia, continuous $95 \% \mathrm{O}_{2}$ exposure was achieved in a Plexiglas chamber $(70 \times 60 \times 30 \mathrm{~cm})$ by a flow-through system. The oxygen level inside the Plexiglas chamber was monitored continuously with a Ceramatec (MAXO2) oxygen analyzer. Carbondioxide $\left(\mathrm{CO}_{2}\right)$ concentration was kept below $0.5 \%$ using a gas monitor (Apex, BW Technologies, Lincolnshire, IL). Temperature and humidity were maintained at $22^{\circ} \mathrm{C}-25^{\circ} \mathrm{C}$ and $60 \%-70 \%$, respectively.

Intraperitoneal (i.p.) injections of saline $(4 \mathrm{ml} / \mathrm{kg})$ in normoxia and hyperoxia groups and those of VPA (30 mg/kg; Sigma-Aldrich, St. Louis, MO) in hyperoxia+VPA group were performed daily from the 1st day of life (P1) throughout P10. Pups in each group were weighed daily and weights were recorded. 


\section{Lung Tissue Preparation}

Pups were sacrificed at P10 under deep anesthesia and all efforts were performed to minimize suffering. Right lungs of rat pups were excised and snap frozen for analyzing tissue cytokines; HDAC activity; expressions of acetylated H3 and H4, TGF 33 , phosphoSMAD2 and active Caspase-3; as well as oxidant/antioxidant enzyme activities and MDA content, while left lungs were perfused for histopathologic and immunohistochemical evaluation after ligation of the right main bronchus.

\section{Histopathologic and Immunohistochemical Evaluation of the Lungs}

Left lungs were fixed by perfusion with $0.1 \mathrm{M}$ phosphate buffered saline (PBS; $\mathrm{pH} 7.4$ ) containing 4\% paraformaldehyde (PFA). Trachea was ligated with a surgical suture, and lungs were incubated in fresh 4\% PFA-PBS solution on ice for 4-5 h. The lungs were paraffin-embedded for obtaining $5 \mu \mathrm{m}$ sections which were then mounted onto poly-Llysine-coated slides (Paul Marienfeld GmbH\&Co., Lauda-Konigshofen, Germany), stained with standard haematoxylin-eosin and Masson's trichrome techniques for histopathologic evaluations and with $A B C$ technique for lamellar body membrane protein (LBMP) expression as described previously [15-17].

Apoptosis was evaluated by Terminal Deoxynucleotidyl Transferase dUTP Nick End Labeling (TUNEL) technique using in situ cell death detection POD kit (Roche Molecular Biochemicals, Mannheim, Germany) as described previously [15].

\section{Western Blot Analyses}

TGF $\beta$ isoforms $(\beta 1, \beta 2, \beta 3)$ play a role in normal tissue repair following lung injury [18]. The intracellular signaling pathway of TGF $\beta$ receptors is mediated by a family of transcription factors, called SMAD proteins. The receptor-regulated SMAD2 and/or SMAD3, in combination with SMAD4 positively regulate the effects of TGF $\beta$ [18]. Therefore, we also evaluated the effect of VPA treatment on TGF $\beta$ pathway and SMAD protein expression in this hyperoxic lung injury model. In addition, members of $\mathrm{Bcl}-2$ gene family are known as the key regulators of cell survival, apoptosis and necrosis and bcl-2 represents an anti-apoptotic protein [19]. Right lungs were homogenized in ice-cold PBS and aliquoted homogenates were used for total protein analysis by bicinchoninic acid (BCA) assay (Thermo Fisher Scientific, Rockford, IL). Aliquots of homogenates were used for determining specific proteins using antibodies against active Caspase-3 (Abcam, Cambridge, MA), bcl-2 (Cell Signaling Technology, Danvers, MA), TGF 1 (Thermo Scientific, Rockford, IL), TGFB3 (Thermo Scientific, Rockford, IL), phospho-SMAD2 (pSer465/467; Thermo Scientific, Rockford, IL), acetyl-histone H3 (Cell Signaling Technology, Danvers, MA) and acetyl-histone H4 (Millipore, Billerica, MA) as described previously [15]. Equal protein loading was confirmed by incubating the stripped membranes with structural protein $\beta$-actin (Abcam, Cambridge, MA). 
HDAC Activity

HDAC activity was assayed using a colorimetric detection kit (Upstate, Temecula, CA) according to the manufacturer's instructions. Absorbances were detected at $405 \mathrm{~nm}$ and results were expressed as the percentage of Normoxia group.

\section{Biochemical analyses}

Cell-free supernatants of lung tissue homogenates were used for determinig lung tissue pro-inflammatory cytokine (TNF- $\alpha$, IL- 6 and IL-1 $\beta$ ) contents by specific enzyme-linked immunosorbent assay (ELISA) kits (R\&D Systems, Minneapolis, MN) [17].

Activities of superoxide dismutase (SOD), glutathione peroxidase (GSH-Px) and myeloperoxidase (MPO), as well as malondialdehyde (MDA) content of lung tissues were measured by spectrophotometric (UV-1700, Shimadzu, Japan) analyses [15].

\section{Statistics}

Statistical analyses were performed using SPSS 16.0 software (IBM Corporation, Armonk, NY). Data were expressed as mean \pm standard error of means (SEM). Normal distributions of data were graphically examined with Shapiro-Wilk test. Categorical variables were compared with Chi square test. Immunohistochemical scores were assessed by Kruskal Wallis test. Treatment groups were compared using One-Way ANOVA and significance was determined using post-hoc Tukey test or Bonferroni's correction for multiple comparisons where applicable. $p<0.05$ was considered statistically significant.

\section{Results}

We started the experiment with 10 rat pups in each group. No pups died in Normoxia or Normoxia+VPA groups throughout the study period. On the other hand, one pup in each Hyperoxia or Hyperoxia+VPA group died on the $2^{\text {nd }}$ or the $3^{\text {rd }}$ postnatal days, respectively. No significant difference was found in terms of survival rate among experimental groups ( $p>0.05)$ and our analyses were not affected by the number of surviving pups. A survival curve has been presented in Fig 1. 


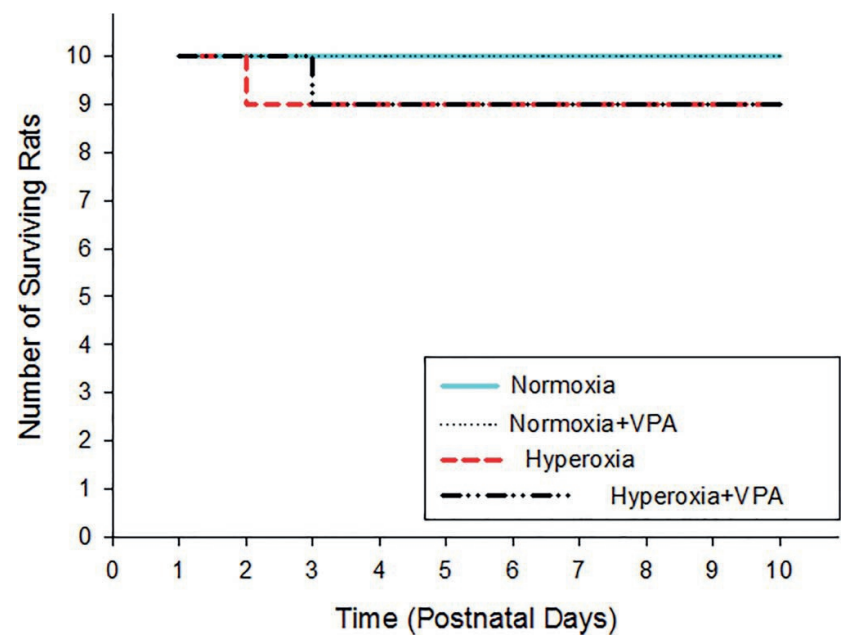

Fig 1. Number of surviving rat pups in experimental groups. doi:10.1371/journal.pone.0126028.g001

The well-being of the rat pups was, in part, assessed with the weight gain during the experiment. Mean birth weights of pups in Normoxia, Normoxia+VPA, Hyperoxia and

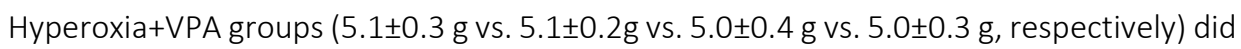
not differ significantly. At the end of the experiment, the mean body weight of pups in Hyperoxia group $(12.4 \pm 1.2 \mathrm{~g})$ was significantly $(p<0.05)$ lower than that of pups in

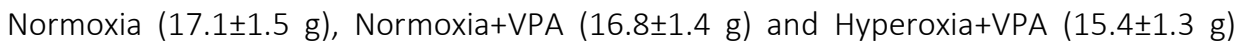
groups (Fig 2). The weight of the Hyperoxia+VPA group was not different to the Normoxia and Normoxia+VPA group.

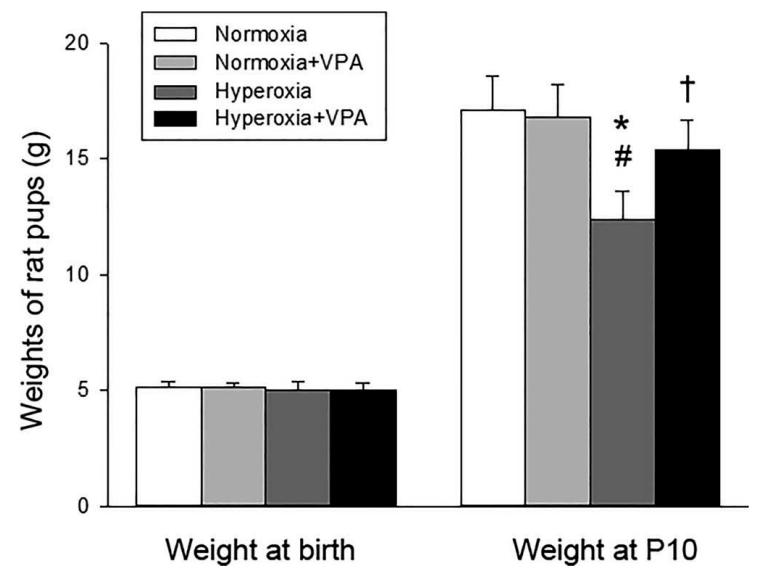

Fig 2. Bar graph depicting body weights of rat pups at birth and $P 10 .{ }^{*} p<0.05$ compared to Normoxia group; $\# p<0.05$ compared to Normoxia+VPA group; and $+p<0.05$ compared to Hyperoxia group using One-Way ANOVA followed by post-hoc Tukey test. doi:10.1371/journal.pone.0126028.g002 
The structure of the lung was assessed by histological examination. The mean histopathologic grade of lung injury in pups in Hyperoxia group was significantly $(p<0.001)$ greater than that in Normoxia and Normoxia+VPA groups; VPA treatment significantly improved histological grading of lung injury compared with Hyperoxia group (Figs $3 \& 4 A$ ). Thickening of the alveolar septi or cell infiltration was not observed in Normoxia, Normoxia+ VPA or Hyperoxia+VPA groups but in the Hyperoxia group (Fig 3). Masson's trichrome-stained sections showed cell infiltration, edema and fibrosis in the Hyperoxia group but not in Normoxia, Normoxia+VPA and Hyperoxia+VPA groups (Fig 3). Mean radial alveolar count, reflecting the number of intact alveoli, was significantly $(p<0.001)$ decreased in Hyperoxia group compared with Normoxia and Normoxia+VPA groups, while it was significantly greater in Hyperoxia+VPA group $(p<0.05)$ compared to Hyperoxia group (Figs 3 \& 4B). Similarly, the decrease in mean LBMP expression $(p<0.001)$ in Hyperoxia group was significantly recovered by VPA treatment ( $p<0.001)$ (Figs $3 \& 4 C$ ). The remodeling of the injured lung was assessed by evaluation of apoptosis including the number of apoptotic cells as well as expressions of active caspase- 3 and bcl-2. When compared with the Normoxia and Normoxia+VPA groups, number of TUNEL (+) cells (Figs $3 \& 4 D$ ) and active Caspase-3 expression (Fig 5A) were significantly increased in Hyperoxia group ( $p<0.001$ and $p<0.001$, respectively) while VPA treatment significantly decreased number of TUNEL $(+)$ cells $(p<0.001)$ (Figs $3 \& 4 D$ ) and active Caspase-3 expression $(p<0.05)$ (Fig 5A). In contrast to active Caspase-3, levels of bcl-2, an anti-apoptotic protein, were significantly decreased in Hyperoxia group $(p<0.001)$ compared with the Normoxia and Normoxia+VPA groups, while bcl-2 was significantly increased in Hyperoxia+ VPA group in comparison with Hyperoxia group ( $p<0.05)$ (Fig 5B).

Inflammation was assessed by levels of IL-1 $\beta, I L-6$, TNF- $\alpha$ and by MPO activity as a marker of neutrophil invasion. Levels of IL-1 $\beta, I L-6$ and TNF- $\alpha$ in the lung tissues were increased in Hyperoxia group compared with the Normoxia and Normoxia+VPA groups $(p<0.05$ for all), whereas VPA treatment significantly reduced hyperoxia-induced elevations of IL-1 $\beta, I L-6$ and TNF- $\alpha$ ( $p<0.05$ for all) (Table 1 ). MPO activity was found to be increased by hyperoxia, whereas VPA treatment decreased it in hyperoxia (Table 1). Expressions of TGF $\beta 1$ and TGF 33 (Fig 6A \& 6B) and phospho-SMAD2 (Fig 6C) in Hyperoxia group were significantly $(p<0.05)$ greater compared with Normoxia and Normoxia+VPA groups, whereas expressions of both proteins were reduced by VPA treatment $(p<0.05)$. 


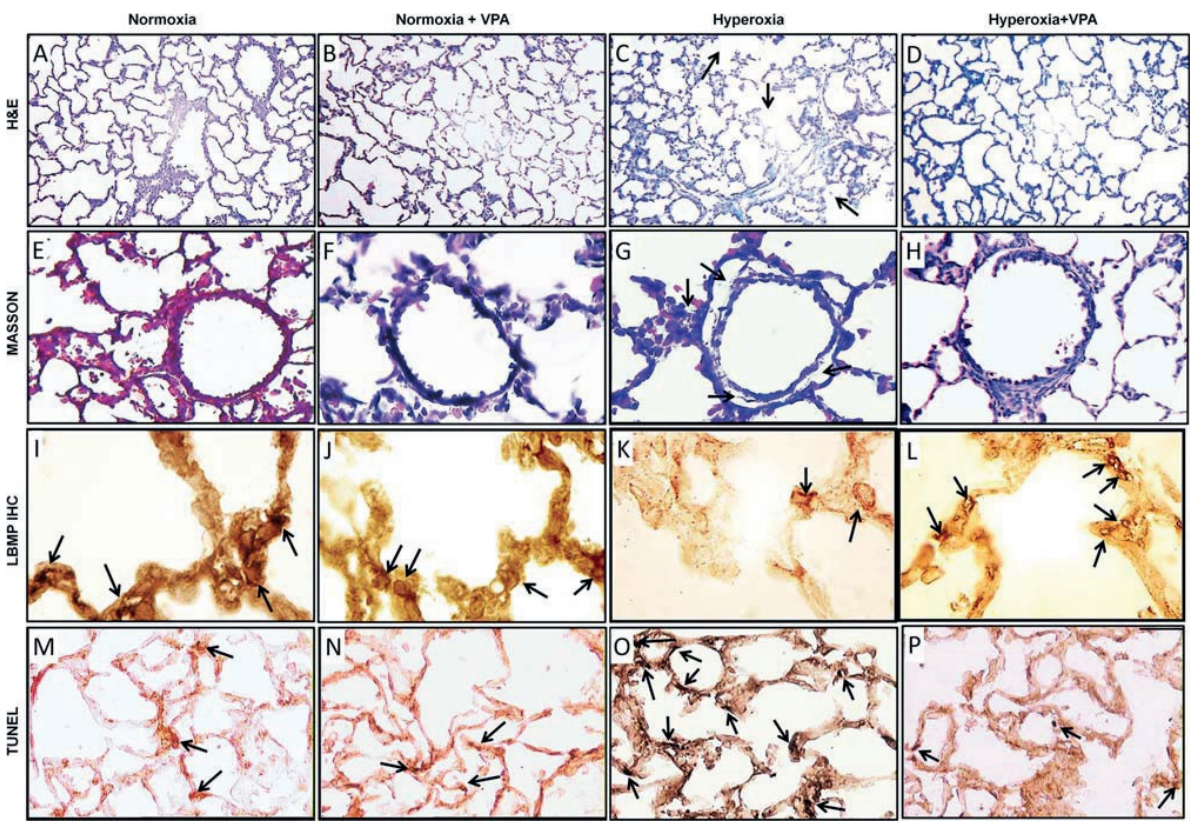

Fig 3. Histological examination of lung tissues by Haematoxylin-eosin (A-D, 100X magnification), Masson's trichrome (E-H, 400X magnification), lamellar body membrane protein (LBMP) (I-L, 1000X magnification), and TUNEL-DAB stainings (M-P, 200X magnification) for Normoxia, Normoxia+VPA, Hyperoxia and Hyperoxia+VPA groups. Representative images show severe alveolar damage (panel C), cell infiltration and edema (panel G, arrow) in Hyperoxia group. Thickening of the alveolar septi or cell infiltration was not observed in Normoxia, Normoxia+VPA and Hyperoxia+VPA groups and panels A, B, D, E, F and $\mathrm{H}$ shows healthier and intact lung parenchymal appearance compared to hyperoxia group. Black arrows indicate positive immunoreactivity for LBMP and TUNEL (+) cells in panels I-P.
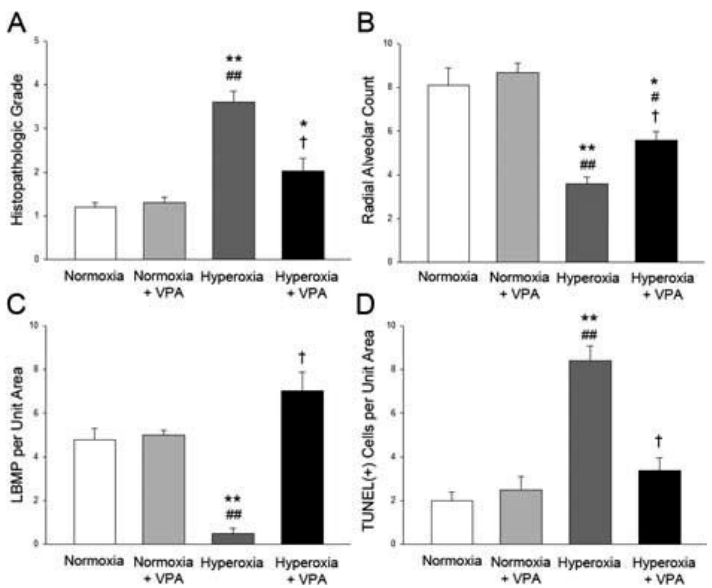

Fig 4. Bar graphs depicting Histopathologic Grade (A), Radial Alveolar Count (B), LBMP per Unit Area (C) and TUNEL (+) Cells per Unit Area (D) in lung tissues of rat pups. ${ }^{*} p<0.05$ and ${ }^{* *} p<0.001$ compared to Normoxia group; \#p<0.05 and \#\#p<0.001 compared to Normoxia+VPA group; and $+p<0.05$ and $++p<0.001$ compared to Hyperoxia group using One-Way ANOVA followed by post-hoc Tukey test. 
A

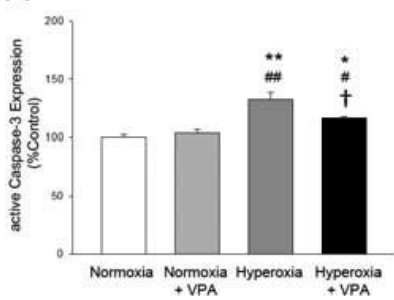

B

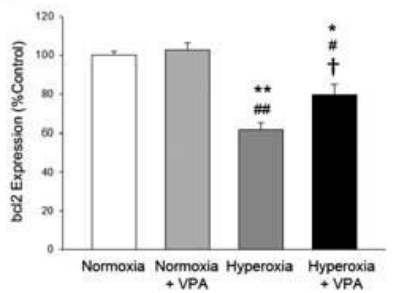

C

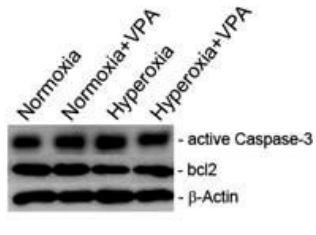

Fig 5. Bar graphs depicting active Caspase-3 (A) and bcl2 (B) expressions in lung tissues of rat pups. Panel C depicts representative bands for each protein, including $\beta$-Actin, the protein which was used as a loading control for western blotting. ${ }^{*} p<0.05$ and ${ }^{* *} p<0.001$ compared to Normoxia group;

$\# p<0.05$ and \#\#p<0.001 compared to Normoxia+VPA group; and $+p<0.05$ compared to Hyperoxia group using One-Way ANOVA followed by post-hoc Tukey test.

A

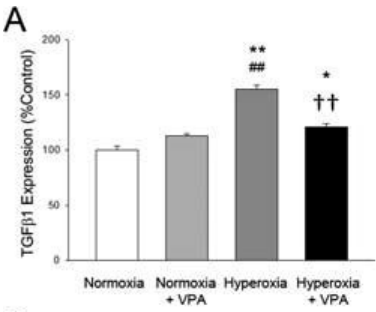

C

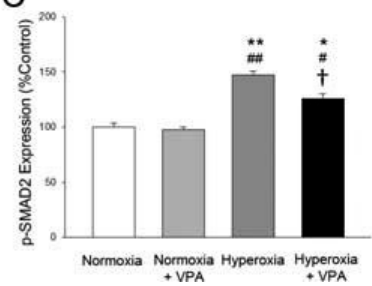

B

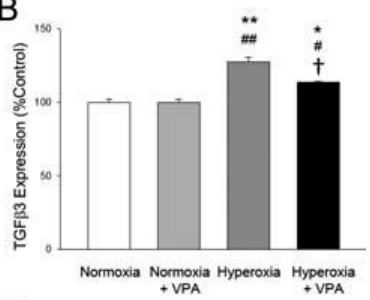

D

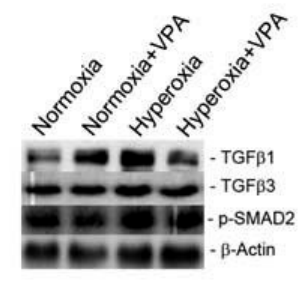

Fig 6. Bar graphs depicting TGF $\beta 1$ (A), TGFB3 (B) and phospho-SMAD2 (C) expressions in lung tissues of rat pups. Panel $D$ depicts representative bands for each protein, including $\beta$-Actin, the protein which was used as a loading control for western blotting. ${ }^{*} p<0.05$ and ${ }^{* *} p<0.001$ compared to Normoxia group; \#p<0.05 and $\# \# p<0.001$ compared to Normoxia+VPA group; and $\uparrow p<0.05$ and $+\uparrow p<0.001$ compared to Hyperoxia group using One-Way ANOVA followed by post-hoc Tukey test. 
The maturity of the antioxidant enzyme systems was assessed by oxidant and antioxidant enzyme activities. The decreases in lung tissue GSH-Px and SOD activities in Hyperoxia group were prevented by VPA in the Hyperoxia+VPA group (Table 1). Similarly, the increases by hyperoxia of lung tissue MDA levels were significantly reduced in Hyperoxia+VPA group (Table 1).

HDAC activity was analyzed in order to test our hypothesis that VPA administration affected epigenetic regulation of pulmonary inflammation in this hyperoxic lung injury model. HDAC activity in lung tissues was found to be significantly enhanced $(p<0.05)$ in Hyperoxia group compared to Normoxia and Normoxia+VPA groups. VPA treatment reduced the increased HDAC activity significantly ( $p<0.05$ ) (Fig 7A). Consistently, acetylhistone $\mathrm{H} 3$ and $\mathrm{H} 4$ protein expressions in Hyperoxia+VPA group were significantly $(p<0.001)$ increased compared with Hyperoxia group (Fig 7B \& 7C, respectively).

Table 1. Comparison of lung tissue pro-inflammatory cytokine levels and superoxide dismutase (SOD), glutathione peroxidase (GSH-Px) and myeloperoxdase (MPO) activities and malonedialdehyde (MDA) content in all groups.

\begin{tabular}{lllll}
\hline $\begin{array}{l}\text { Cytokine Levels } \\
(\mathrm{pg} / \mathrm{ml})\end{array}$ & $\begin{array}{l}\text { Normoxia } \\
\text { Group }\end{array}$ & $\begin{array}{l}\text { Normoxia+VPA } \\
\text { Group }\end{array}$ & $\begin{array}{l}\text { Hyperoxia } \\
\text { Group }\end{array}$ & $\begin{array}{l}\text { Hyperoxia+VPA } \\
\text { Group }\end{array}$ \\
\hline IL-1 $\beta$ & $86.2 \pm 6.4$ & $89.4 \pm 7.6$ & $129.2 \pm 10.1^{*}$ & $98.6 \pm 7.5^{\dagger}$ \\
IL-6 & $36.1 \pm 5.7$ & $37.3 \pm 5.4$ & $58.4 \pm 5.1^{*}$ & $42.5 \pm 4.7^{\dagger}$ \\
TNF- $\alpha$ & $101.6 \pm 18.4$ & $106.7 \pm 12.6$ & $242.5 \pm 32.9^{*}$ & $141.7 \pm 19.2^{\dagger}$ \\
Biochemical Analyses & & & & \\
GSH-Px (U/mg protein) & $13.2 \pm 1.4$ & $12.4 \pm 1.2$ & $4.1 \pm 1.4^{*}$ & $9.5 \pm 1.3^{\dagger}$ \\
SOD (U/mg protein) & $115.8 \pm 10.6$ & $118.6 \pm 9.1$ & $60.5 \pm 3.1^{* *}$ & $90.8 \pm 8.4 \ddagger$ \\
MPO (U/g protein) & $25.7 \pm 3.6$ & $31.7 \pm 4.4$ & $82.7 \pm 4.3^{* *}$ & $56.5 \pm 5.2 \ddagger$ \\
MDA (nmol/g protein) & $24.6 \pm$ & $26.8 \pm 4.1$ & $80.4 \pm 4.7^{* *}$ & $47.6 \pm 4.1^{\dagger}$ \\
\hline
\end{tabular}

$* p<0,05$ and

** $p<0,01$ compared to Normoxia group; and

$+p<0,05$ and

$\ddagger p<0,01$ compared to Hyperoxia group using One-Way ANOVA followed by post-hoc Tukey test. 

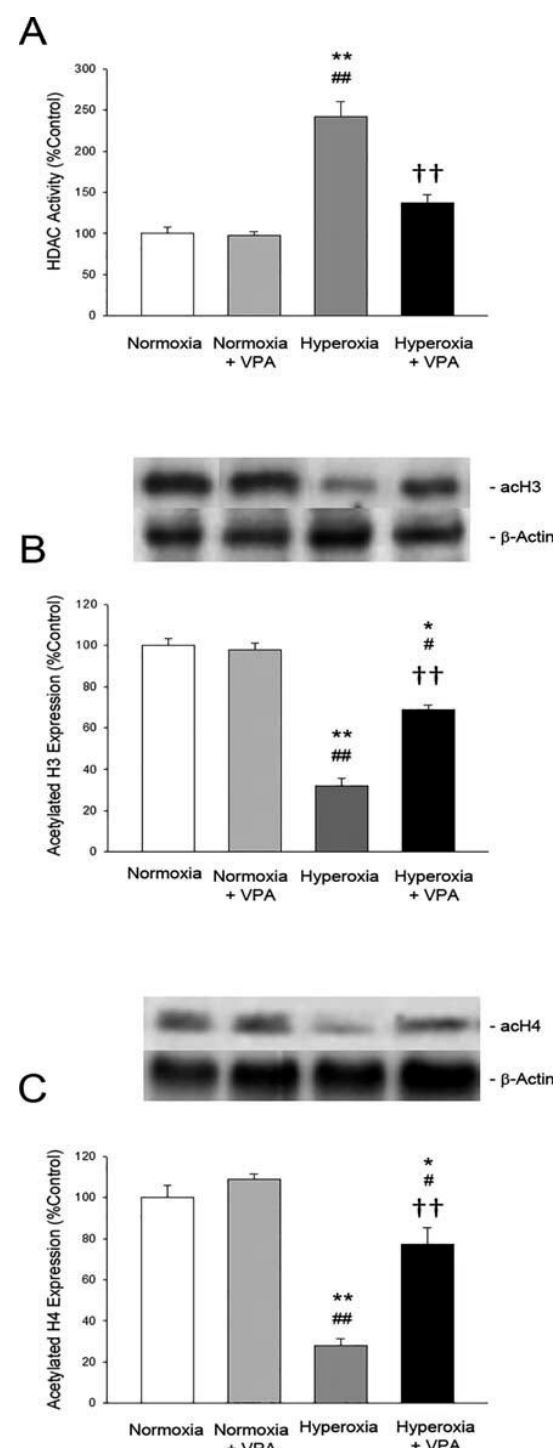

Fig 7. Bar graphs depicting histone deacetylase (HDAC) activity (A) as well as acetylated histone $\mathrm{H3}$ and acetylated histone $\mathrm{H} 4$ (C) protein expressions in lung tissues of rat pups. ${ }^{*} \mathrm{p}<0.05$ and ** $<<0.001$ compared to Normoxia group; \#p<0.05 and \#\#p<0.001 compared to Normoxia+VPA group; and $++p<0.001$ compared to Hyperoxia group using One-Way ANOVA followed by post-hoc Tukey test. doi:10.1371/journal.pone.0126028.g007 


\section{Discussion}

The present data showed that systemic administration of VPA to rat pups under hyperoxic conditions provided significant protection against lung damage in a neonatal rat model of hyperoxic lung injury and preserved body growth. The effect of VPA was in part mediated by epigenetic modulation of inflammation. We conclude that VPA treatment exhibits anti-inflammatory, anti-apoptotic and anti-fibrotic effects in hyperoxic lung injury model in neonatal rats.

Chronic inflammation is the hallmark of hyperoxic lung damage and BPD, which were shown to alter HAT/HDAC ratio, resulting in histone hypoacetylation [9]. Given the problems associated with the use of postnatal steroids for the prevention of BPD with respect to long term neurodevelopmental outcome, we successfully tested a different approach of epigenetic modulation for postnatal chronic pulmonary inflammation. Recent data established that VPA might lead to hyperacetylation of histones by directly inhibiting HDACs. Since histone acetylation and deacetylation might play an important role in the pathogenesis of inflammatory lung diseases $[9,20]$, HDAC inhibitors were already suggested to provide protection against lung fibrosis to altered gene expression after hyperacetylation $[7,21]$. Therefore, we used VPA for this purpose and showed that VPA affected acetylation process in the lung, prevented apoptosis, and decreased inflammation.

VPA attenuated hyperoxic lung injury by improving the histopathological score and preserving alveolarization in the present study. Our data are in accordance with a previous report which showed that VPA improved histopathological injury in lung, liver and kidney, and ameliorated multiple organ dysfunction caused by LPS induced septic shock [14]. In addition, we showed that VPA significantly increased lamellar membrane protein expression and mean radial alveolar count as an evidence for the preservation of alveolarization in hyperoxic lung injury. The structural changes were associated with elevated expressions of TGFB3 and SMAD2 proteins in lung tissue samples of rat pups exposed to hyperoxia and these elevations were restored by VPA treatment which is in line with clinical data where increased levels of TGF $\beta$ isoforms in bronchoalveolar lavage fluid of preterm infants were associated with the severity of BPD [22]. In addition, our data showed that VPA decreased apoptosis by reducing both the number of TUNEL (+) cell count and active caspase-3 expression in lung tissue. Exposure of neonatal rat lung to hyperoxia resulted in apoptosis which was associated with the early rise in proapoptotic proteins that overcome the anti-apoptotic activity of $\mathrm{Bcl}-2$ [23]. Although the protective effects of HDAC inhibitors in experimental models of inflammation were found to be associated with increased expression of bcl-2 protein family, the precise mechanism of this up-regulation has not been defined [24]. We had similar findings in our study in which VPA administration increased bcl-2 expression in neonatal rats subjected to hyperoxia. To the best of our knowledge, this is the first study to present data on the effect of VPA on bcl-2 expression in a neonatal rat model of hyperoxia. The mechanism 
by which VPA administration increased bcl-2 expression in neonatal rats under hyperoxic conditions is yet to be determined. On the other hand, the effect may involve a regulation by $\mathrm{p53}$. It was shown in cancer cells that the tumor suppressor p53 induces apoptosis by inhibiting the anti-apoptotic bcl-2 [25] as well as the pro-apoptotic bax protein [26]. In addition, recent studies report a direct effect of HDAC inhibitors on p53, again in cancer cells [27-29]. Accordingly, HDAC inhibitors induce p53 expression, which in turn inhibits the expression of bcl-2 to promote apoptotic death in certain cancer types. However, we found in our study that VPA administration increased the expression of the anti-apoptotic bcl-2 while decreasing the expression of the pro-apoptotic Caspase-3 protein. Therefore, it is reasonable to speculate that the regulation of bcl-2 in hyperoxic lung may be different from that in cancer cells. Nevertheless, our study was not designed to unravel the mechanism of bcl-2 regulation by VPA which must be investigated by future studies. All these results suggest that protective effect of VPA on fibrotic tissue remodeling involves reduction of TGF $\beta / S M A D$ expression and also apoptosis. The positive impact of VPA on alveolar lung development and apoptotic mechanisms must be evaluated in future studies.

The structural benefit was associated with preserved GSH-Px and SOD activities and reduced tissue MDA content, MPO activity and levels of pro-inflammatory cytokines suggesting that one of the mechanisms by which VPA prevents hyperoxic lung injury is enhancement of antioxidant activity and reduction of lipid peroxidation and inflammation. Similarly, VPA showed protection from lung inflammation by decreasing serum IL-6 and TNF- $\alpha$ levels, as well as MPO activity significantly in pulmonary tissue by amelioration of lung damage in a rat model of septic shock [14] or acute lung injury [30]. The systemic effects on pro-inflammatory cytokine concentrations may have resulted in one of the most interesting effects of VPA. VPA promoted growth in the rat pups that were exposed to hyperoxia. Since malnutrition is known to worsen BPD by compromising lung growth [31], we consider VPA as an interesting candidate for the prevention of BPD. However, it is difficult to translate experimental studies into clinical setting [32]. First of all, our study is limited with the lack of data on antenatal steroids, the effects of which on epigenetic regulation, lung growth, inflammation and TGF- $\beta$ signaling have not been taken into account in our model. Although the effect of antenatal steroids on epigenetic mechanisms in the lung is not known, intrauterine inflammation induced TGF- $\beta$ signaling was shown to be inhibited by antenatal steroids [33]. It was also reported that the order of exposure to inflammation or maternal steroids had large and different effects on fetal lung maturation [34]. Therefore, future studies are warranted that aim to investigate the role of antenatal steroids on epigenetic changes in lung development. Second of all, VPA is a potent drug which has been shown to induce cognitive deficits and the risk of autism after in utero exposure $[32,35]$. In addition, VPA in neonates may lead to progressive hyperammonemia, cerebral edema, and diminished level of consciousness and these potential adverse effects hamper the clinical usage of VPA in this high risk group [36]. However, on the other hand, VPA has been used in neonates with refractory convulsions 
[37]. Therefore, experimental and clinical data regarding the safety of VPA usage in neonates are required before its clinical applicability. In addition, postnatal VPA usage in clinical care warrants long term neurodevelopmental outcome studies in neonates.

In conclusion, VPA treatment improved alveolarization by preserving histopathological structure, radial alveolar count and lamellar body membrane protein expression in the alveoli as well as it reduced fibrosis, TUNEL (+) cell counts, active Caspase-3 expression and inflammation, and enhanced antioxidant activity while decreasing lipid peroxidation in the hyperoxic lung. In addition, VPA reduced HDAC activity and enhanced acetyl-histone $\mathrm{H} 4$ protein expression in the injured lung tissue suggesting the involvement of the inhibitory action of VPA on HDAC activity. To the best of our knowledge, this is the first study that showed preventive effects of VPA, a HDAC inhibitor, in a neonatal rat model of hyperoxic lung injury and reduced body growth. Our data suggest that VPA may be beneficial in treatment of hyperoxic lung injury in preterm infants in case its effectiveness and safety is proven in human studies.

\section{Author Contributions}

Conceived and designed the experiments: M. Cetinkaya M. Cansev FC CT FEC BWK SUS. Performed the experiments: M. Cansev FC CT FEC. Analyzed the data: M. Cansev IMK EOY. Contributed reagents/materials/analysis tools: M. Cansev IMK EOY. Wrote the paper: M. Cetinkaya M. Cansev BWK. 


\section{References}

1. Gien J, Kinsella JP. Pathogenesis and treatment of bronchopulmonary dysplasia. Curr Opin Pediatr. 2011; 23: 305-313. doi: 10.1097/MOP.0b013e328346577f PMID: 21494147

2. Madurga A, Mizikova I, Ruiz-Camp J, Morty RE. Recent advances in lung development and the pathogenesis of bronchopulmonary dysplasia. Am J Physiol Lung Cell Mol Physiol. 2013; 35: L893-905. doi:10.1152/ajplung.00267.2013 PMID: 24213917

3. Pfister RH, Soll RF. Pulmonary care and adjunctive therapies for prevention and amelioration of bronchopulmonary dysplasia. Neoreviews 2011; 12: e635.

4. Doyle DW, Ehrenkranz RA, Halliday HL. Early (<8 days) postnatal corticosteroids for preventing chron- ic lung disease in preterm infants. Cochrane Database Syst Rev. 2014; 5: CD001146. doi: 10.1002/ 14651858.CD001146.pub4 PMID: 24825456

5. Malaeb SN, Stonestreet BS. Steroids and injury to the developing brain: net harm or net benefit? Clin Perinatol. 2014; 41: 191-208. doi: 10.1016/j.clp.2013.09.006 PMID: 24524455

6. Doyle DW, Ehrenkranz RA, Halliday HL. Late (>7 days) postnatal corticosteroids for chronic lung dis- ease in preterm infants. Cochrane Database Syst Rev. 2014; 13: CD001145).

7. Tratter KW, Archer TK. Nuclear receptors and chromatin remodeling machinery. Mol Cell Endocrinol. 2007; 265-266: 162-167.

8. Shanmugam MK, Sethi G. Role of epigenetics in-inflammation associated diseases. Subcell Biochem. 2013; 61: 627-657. doi: 10.1007/978-94-007-4525-4_27 PMID: 23150270

9. Barnes PJ, Adcock IM, Ito K. Histone acetylation and deacetylation: importance in inflammatory lung diseases. Eur Respir J. 2005; 25: 552-563. PMID: 15738302

10. Cohen J, Van Marter L, Sun Y, Allred E, Leviton A, Kohane IS. Perturbation of altered gene expression of the chromatin remodeling pathway in premature infants at risk for bronchopulmonary dysplasia. Ge- nome Biol. 2007; 8: R210. PMID: 17916252

11. Göttlicher M, Minucci S, Zhu P, Krämer OH, Schimpf A, Giavara S, et al. Valproic acid defines a novel class of HDAC inhibitors inducing differentiation of transformed cells. EMBO J. 2001; 20: 6969-6978. PMID: 11742974

12. Kim HJ, Rowe M, Ren M, Hong JS, Chen PS, Chuang DM. Histone deacetylase inhibitors exhibit antiinflammatory and neuroprotective effects in a rat permanent ischemic model of stroke: multiple mechanisms of action. J Pharmacol Exp Ther. 2007; 321: 892-901. PMID: 17371805

13. Glauben R, Batra A, Fedke I, Zeitz M, Lehr HA, Leoni F, et al. Histone hyperacetylation is associated with amelioration of experimental colitis in mice. J Immunol. 2006; 176: 5015-5022. PMID: 16585598

14. Shang Y, Jiang YX, Ding ZJ, Shen AL, Xu SP, Yuan SY, et al. Valproic acid attenuates the multiple- organ dysfunction in a rat model of septic shock. Chin Med J. (Eng) 2010; 123: 2682-2687. PMID: 21034653

15. Cetinkaya M, Cansev M, Kafa IM, Tayman C, Cekmez F, Canpolat FE, et al. Cytidine 5'-diphosphocho- line (CDP-choline) ameliorates hyperoxic lung injury in a neonatal rat model. Pediatr Res. 2013; 74: 26-33. doi: 10.1038/pr.2013.68 PMID: 23598810

16. Ozdulger A, Cinel I, Koksel O, Cinel L, Avlan D, Unlu A, et al. The protective effect of N-acetylcysteine on apoptotic lung injury in cecal ligation and puncture-induced sepsis model. Shock 2003; 19: 366-372. PMID: 12688549

17. Askenazi SS, Perlman M. Pulmonary hypoplasia: lung weight and radial alveolar count as criteria of diagnosis. Arch Dis Child 1979; 54: 614-618. PMID: 507916

18. Massague J, Seoane J, Wotton D. Smad transcription factors. Genes Dev. 2005; 19: 2783-2810. PMID: 16322555

19. O'Reilly MA, Staversky RJ, Huyck HL, Watkins RH, LoMonaco MB, D'Angio CT, et al. Bcl-2 family gene expression during severe hyperoxia induced lung injury. Lab Invest 2000; 80: 1845-1854. PMID:11140697

20. Adcock IM, Tsaprouni L, Bhavsar P, Ito K. Epigenetic regulation of airway inflammation. Curr Opin Immunol. 2007; 19: 694-700. PMID: 17720468 
21. Albertine K, Amundsen S, Metcalfe D, Wint A. Histone acetylation in the lung is affected by ventilation mode in preterm lambs. Pediatric Academic Societies' Annual Meeting, Honolulu 2008; 3060.2.

22. Kotecha S, Wangoo A, Silverman M, Shaw RJ. Increase in the concentration of transforming growth factor beta-1 in bronchoalveolar lavage fluid before development of chronic lung disease of prematurity. J Pediatr. 1996; 128: 464-469. PMID: 8618178

23. Husari AW, Dbaibo GS, Bitar H, Khayat A, Panjarian S, Nasser M, et al. Apoptosis and the activity of ceramide, $\mathrm{Bax}$ and $\mathrm{Bcl}-2$ in the lungs of neonatal rats exposed to limited and prolonged hyperoxia. Resp Res. 2006; 7:100

24. Shein NA, Shohami E. Histone deacetylase inhibitors as therapeutic agents for acute central nervous system injuries. Mol Med. 2011; 17: 448-456. doi: 10.2119/molmed.2011.00038 PMID: 21274503

25. Miyashita T, Harigai M, Hanada M, Reed JC. Identification of a p53-dependent negative response ele- ment in the bcl-2 gene. Cancer Res. 1994; 54: 3131-3135. PMID: 8205530

26. Miyashita T, Reed JC. Tumor suppressor p53 is a direct transcriptional activator of the human bax gene. Cell 1995; 80: 293-299. PMID: 7834749

27. Hemann MT, Lowe SW. The p53-Bcl-2 connection. Cell Death Differ. 2006; 13: 1256-1259. PMID: 16710363

28. Feng D, Wu J, Tian Y, Zhou H, Zhou Y, Hu W, et al. Targeting of histone deacetylases to reactivate tu- mour suppressor genes and its therapeutic potential in a human cervical cancer xenograft model. PLoS One 2013; 8: e80657. doi: 10.1371/journal.pone.0080657 PMID: 24260446

29. Sonnemann J, Marx C, Becker S, Wittig S, Palani CD, Krämer OH, et al. p53-dependent and p53-independent anticancer effects of different histone deacetylase inhibitors. Br J Cancer 2014; 110: 656-667. doi: 10.1038/bjc.2013.742 PMID: 24281001

30. Kim K, Li Y, Jin G, Chong W, Liu B, Lu J, et al. Effect of valproic acid on acute lung injury in a rodent model of intestinal ischemia reperfusion. Resuscitation 2012; 83: 243-248. doi: 10.1016/j. resuscitation.2011.07.029 PMID: 21824465

31. Biniwale MA, Ehrenkranz RA. The role of nutrition in the prevention and management of bronchopulmonary dysplasia. Semin Perinatol. 2006; 30: 200-208. PMID: 16860160

32. Roullet FI, Lai JK, Foster JA. In utero exposure to valproic acid and autism-a current review of clinical and animal studies. Neurotoxicol Teratol. 2013; 36: 47-56. doi: 10.1016/j.ntt.2013.01.004 PMID:23395807

33. Collins JJ, Kunzmann S, Kuypers E, Kemp MW, Speer CP, Newnham JP, et al. Antenatal glucocorti- coids counteract LPS changes in TGF- $\beta$ pathway and caveolin-1 in ovine fetal lung. Am J Physiol Lung Cell Mol Physiol. 2013; 304: L438-444. doi: 10.1152/ajplung.00251.2012 PMID: 23333802

34. Kuypers E, Collins JJ, Kramer BW, Ofman G, Nitsos I, Pillow JJ. Intra-amniotic LPS and antenatal betamethasone: inflammation and maturation in preterm lamb lungs. Am J Physiol Lung Cell Mol Physiol. 2012; 302: L380-389. doi: 10.1152/ajplung.00338.2011 PMID: 22160306

35. Roullet FI, Wollaston L, Decatanzaro D, Foster JA. Behavioral and molecular changes in the mouse in response to prenatal exposure to the anti-epileptic drug valproic acid. Neuroscience 2010; 170: 514- 522. doi: 10.1016/j.neuroscience.2010.06.069 PMID: 20603192

36. Unal E, Kaya U, Aydin K. Fatal valproate overdose in a newborn baby. Hum Exp Toxicol. 2007; 26: 453-456. PMID: 17623771

37. Tulloch JK, Carr RR, Ensom MH. A systematic review of the pharmacokinetics of antiepileptic drugs in neonates with refractory seizures. J Pediatr Pharmacol Ther. 2012; 17: 31-44. doi: 10.5863/1551- 677617.1.31 PMID: 23118657 



\section{Chapter}

\section{Cytidine 5'-diphosphocholine ameliorates hyperoxic lung injury in a neonatal rat model}

Published as: Cetinkaya M, Cansev M, Kafa IM, Tayman C, Cekmez F, Canpolat FE, Tunc T, Sarici SU. Cytidine 5'-diphosphocholine ameliorates hyperoxic lung injury in a neonatal rat model. Pediatr Res 2013; 74: 26-33. 


\section{Abstract}

\section{Background:}

Bronchopulmonary dysplasia (BPD) is an important cause of morbidity. The aim of this study was to evaluate the preventive effect of cytidine 5 '-diphosphocholine (CDP-choline) treatment on hyperoxic lung injury in a neonatal rat model.

\section{Methods:}

A total of 30 newborn pups were divided into control, hyperoxia, and hyperoxia + CDPcholine groups. After birth, pups in the control group were kept in room air and received saline injections, whereas those in hyperoxia and hyperoxia + CDP-choline groups were exposed to $95 \% \mathrm{O}_{2}$ and received daily injections of saline and CDP-choline throughout postnatal day 10, respectively. Histopathological scoring, radial alveolar count, lamellar body membrane protein expression, fibrosis, proinflammatory cytokine levels, lung tissue and bronchoalveolar lavage (BAL) fluid phospholipid content, and apoptosis were evaluated.

\section{Results:}

Hyperoxia-induced severe lung damage was reduced signify by CDP-choline treatment. Radial alveolar count and lamellar body membrane protein expression were significantly recovered, and the number of terminal deoxynucleotidyl transferase-mediated deoxyuridine triphosphate nick-end labelling-positive cells, active caspase-3 expression, and tissue proinflammatory cytokine levels were decreased by CDP-choline administration. Lung tissue and BAL phospholipid contents showed significant increases after CDP-choline administration.

\section{Conclusion:}

These data show that CDP-choline ameliorates hyperoxic lung injury in a neonatal rat model. It may therefore be suggested that CDP-choline may be a novel therapeutic option for the prevention of BPD. 
Bronchopulmonary dysplasia (BPD) is an important cause of morbidity in preterm infants that results in prolonged hospitalization, discharge from hospital with oxygen therapy, frequent readmission to the hospital with respiratory problems, and increased neurodevelopmental risk (1). Although BPD has a multifactorial etiology, major risk factors for BPD development include preterm birth, lower gestational age and birth weight, need for supplemental oxygen and ventilatory support, oxygen toxicity, decreased host antioxidant defenses, patent ductus arteriosus, and pre- and postnatal infections (2). Therefore, treatment of evolving BPD may be challenging due to the complex balance between these contributing risk factors. Efficacy of parenteral administration of vitamin A or caffeine, the two successful treatments developed to prevent BPD, was supported by evidence from randomized controlled studies (3), whereas many other treatments aiming at preventing BPD have failed to demonstrate a reduction in BPD rates (2). Therefore, newer strategies and pharmacological approaches to prevent BPD are required in order to decrease BPD incidence and severity.

Cytidine 5'-diphosphocholine (CDP-choline), which is composed of cytidine and choline linked by a diphosphate bridge, is an endogenous intermediate in the synthesis of the major membrane phospholipid, phosphatidylcholine (PC) (4). PC is quantitatively the most important phospholipid, accounting for $70-85 \%$ of the total surfactant phospholipids, which are major constituents of surfactant lipids (5). The primary function of surfactant is to decrease surface tension at the air-liquid surface in the alveoli and distal bronchioli, to promote lung expansion during inspiration in order to prevent atelectasis at end expiration. It also plays an important role in pulmonary host defense and local immunomodulation by enhancing the stability of the film that floats on the alveolar linings, facilitating mucociliary transport, exhibiting antioxidant activity with antibacterial/antiviral properties (5). Due to persisting surfactant abnormalities including an ongoing quantitative deficiency in surfactant components, decreased function of endogenous surfactant, and increased surfactant turnover in ventilated preterms with evolving BPD, surfactant therapy was suggested to show benefits in prevention of BPD (6). In addition, the beneficial effects of additional surfactant therapy for prevention of BPD were also evaluated (7).

In addition, in a few studies with conflicting outcomes, CDP-choline was administered parenterally at a dose range of $100-300 \mathrm{mg} / \mathrm{kg} / \mathrm{d}$ to preterm infants to increase the synthesis of lung phospholipids for alleviating the symptoms of respiratory distress syndrome $(8,9)$. However, to the best of our knowledge, no study evaluated the efficacy of CDP-choline for preventing BPD. Therefore, the aim of this experimental study was to investigate the possible protective effect of CDP-choline against hyperoxic lung injury in a neonatal rat model. 


\section{RESULTS}

No significant difference was detected between mean birth weights of pups in the control, hyperoxia, and hyperoxia + CDP-choline groups (5.0 \pm 0.4 vs. $4.9 \pm 0.2$ vs. $5.0 \pm$ $0.3 \mathrm{~g}$, respectively) $(\mathrm{P}>0.05)$. However, the mean body weight of pups in the hyperoxia + CDP-choline group (15.2 $\pm 1.2 \mathrm{~g}$ ) was significantly greater than that of pups in the hyperoxia group (12.2 $\pm 0.9 \mathrm{~g})(\mathrm{P}=0.02)$, but not in the control group (17.4 $\pm 1.6 \mathrm{~g}$; $\mathrm{P}>$ 0.05 ) at end of the study (postnatal day (P)10). During the experimental procedures, two pups in the hyperoxia group and one pup in the hyperoxia + CDP-choline group died. However, no significant difference was found between these two groups in terms of survival $(P>0.05)$.

Severity of lung damage was evaluated between grades 1 and 4 by histopathologic examination (Figures $1 \mathrm{a}-\mathrm{c}$ and 2 ). Thickening of the alveolar septi or cell infitration was not observed in the control and hyperoxia + CDP-choline groups. CDP-choline treatment significantly improved histological grading of lung injury as compared with saline treatment in the hyperoxia group ( $P=0.01$ ) (Figure 1a-c). Masson's trichrome stained sections also showed cell infiltration, edema, and fibrosis in the hyperoxia group, which were not noticeably observed in the control and hyperoxia + CDP-choline groups (Figure $1 d-f)$. Radial alveolar count, reflected by the number of intact alveoli, was also significantly greater in the hyperoxia + CDP-choline group as compared with the hyperoxia group $(\mathrm{P}<0.05)$ (Figure 2$)$.

Although expression of P180 lamellar body membrane protein was decreased in the hyperoxia group, it was recovered by CDP- choline treatment (Figures 1g-i and 2). By contrast, the number of terminal deoxynucleotidyl transferase-mediated deoxyuridine triphosphate nick-end labeling (TUNEL)-positive cells per unit area (Figure 3 ) and active caspase-3 expression ( $209.2 \pm 13.7 \%$ in the hyperoxia group vs. $127.9 \pm 5.6 \%$ in the hyperoxia + CDP-choline group) (Figure 4) were lower in the hyperoxia + CDP-choline group as compared with those in the hyperoxia group $(P<0.05)$. Levels of $\beta$-actin, the protein that was used as a protein- loading control for gel electrophoresis, did not change between the groups.

To further investigate the possible mechanism of action of CDP-choline treatment, lung tissue inflammatory cytokine levels were also analyzed. In the hyperoxia group, levels of interleukin (IL)-1 $\beta$, IL-6, and tumor necrosis factor- $\alpha$ (TNF- $\alpha$ ) were found to be increased as compared with those of the control group $(P<0.05)$, whereas CDP-choline treatment significantly reduced hyperoxia-associated elevations of IL-1 $\beta$ (from $148.9 \pm$ 12.4 to $112.1 \pm 9.4 \mathrm{pg} / \mathrm{ml}$ ), IL-6 (from $43.7 \pm 4.5$ to $28.6 \pm 4.1 \mathrm{pg} / \mathrm{ml}$ ), and TNF- $\alpha$ (from $246.7 \pm 30.9$ to $146.2 \pm 20.7 \mathrm{pg} / \mathrm{ml})(P<0.05)$ (Table 1$)$, suggesting an anti-inflammatory effect of CDP-choline treatment.

To investigate the effect of supplemented CDP-choline on surfactant components, a phospolipid assay was performed in bronchoalveolar lavage (BAL) fluid and lung tissue homogenates. As shown in Table 2, the content of total phospholipids and phospholipid 
classes including PC, phosphatidylglycerol, and disaturated PC (DSPC) significantly decreased in both BAL and lung tissue samples of rat pups exposed to hyperoxia as compared with those in control pups, whereas CDP-choline treatment significantly increased the contents of the measured phospholipid classes (Table 2).

In addition, biochemical analyses revealed that subjection to hyperoxia significantly decreased tissue superoxide dismutase (SOD) and glutathione peroxidase (GSH-Px) activities, whereas it increased tissue malondialdehyde (MDA) content and myeloperoxidase (MPO) activity (Table 3). By contrast, mean tissue SOD and GSH-PX activities were significantly higher in the hyperoxia + CDP-choline group as compared with the hyperoxia group ( $95.6 \pm 6.1$ vs. $63.7 \pm 2.5 \mathrm{U} / \mathrm{mg}$ protein, $\mathrm{P}=0.001$ and $11.5 \pm 1.5$ vs. $5.6 \pm 1.8 \mathrm{U} / \mathrm{g}$ protein, $\mathrm{P}=0.01$, respectively), indicating the efficacy of CDP-choline on decreasing oxidative stress. Mean tissue MDA content and MPO activity (52.9 \pm 3.2 vs. $68.8 \pm 7.4 \mathrm{nmol} / \mathrm{g}$ protein, $\mathrm{P}=0.001$ and $55.5 \pm 6.8 \mathrm{vs} .76 .2 \pm 2.1 \mathrm{U} / \mathrm{g}$ protein, $\mathrm{P}=0.001$, respectively) were significantly lower in the hyperoxia + CDP-choline group as compared with those in the hyperoxia group, suggesting a CDP-choline effect on decreasing lipid peroxidation (Table 3). 


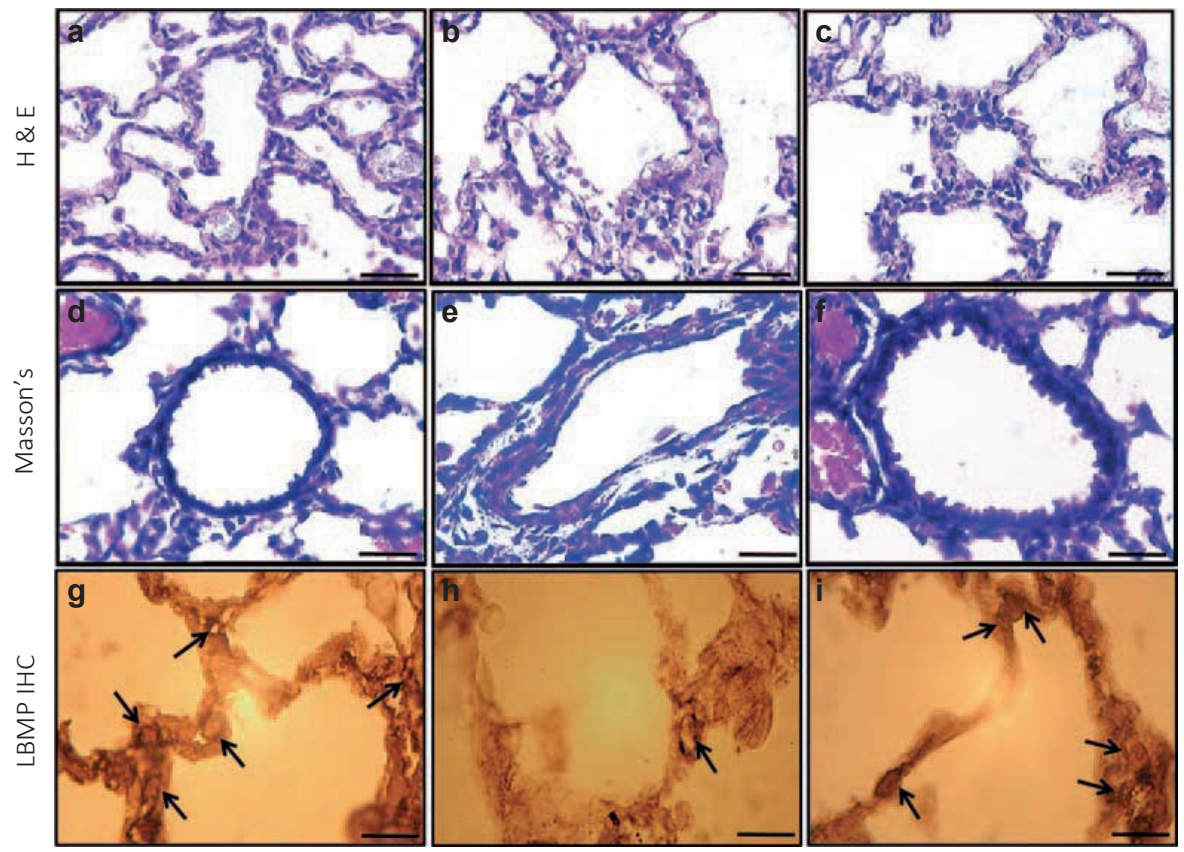

Fig. 1. Representative images of (a-c) hematoxylin-eosin staining, (d-f) Masson's trichrome staining, and (g-i) immunohistochemistry for lamellar body membrane protein. Histological appearance of the lungs in the hyperoxia group is characterized by partial destruction of the lung tissue (in b), cell infiltration, edema, and fibrosis (in b and e), which are not notably observed in the control (in a and d) and hyperoxia + CDP-choline (in $\mathrm{c}$ and f) groups. (g-i) Abundant positive immunohistochemistry for the lamellar body membrane protein for control and hyperoxia + CDP-choline groups (black arrows, DAB-positive lamellar body membrane protein immunoreactivities). Bars, $50 \mu \mathrm{m}$ (in d-f); $25 \mu \mathrm{m}$ (in a-c and g-i). CDP-choline, cytidine 5'-diphosphocholine; DAB, 3,3'-diaminobenzidine; H\&E, hematoxylin-eosin staining; LBMP IHC, lamellar body membrane protein immunohistochemistry.
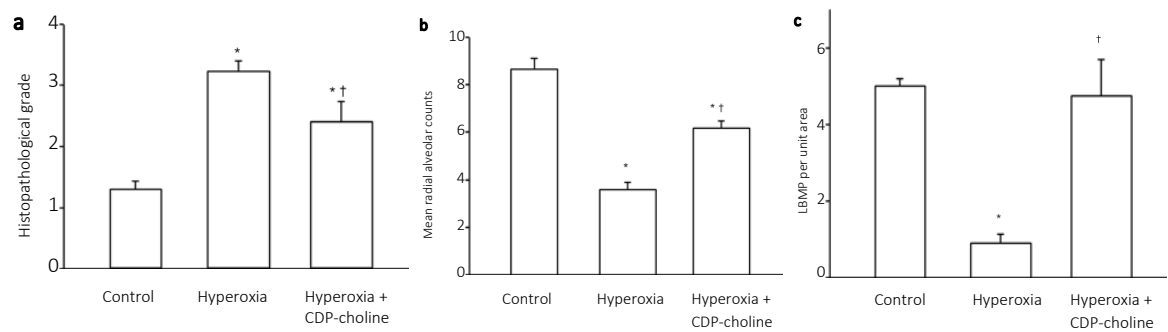

Fig. 2. Bar graphs for (a) histopathologic evaluation, (b) radial alveolar count, and (c) lamellar body membrane protein expression. ${ }^{*} P<0.05$, signifi- cantly different from control group; $+P<0.05$, significantly different from hyperoxia group using one-way ANOVA followed by post hoc Holm-Sidak test. CDP-choline, cytidine 5'diphosphocholine; LBMP, lamellar body membrane protein. 
a

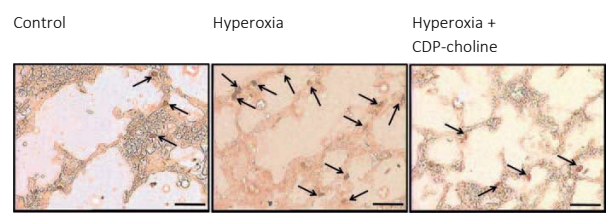

b

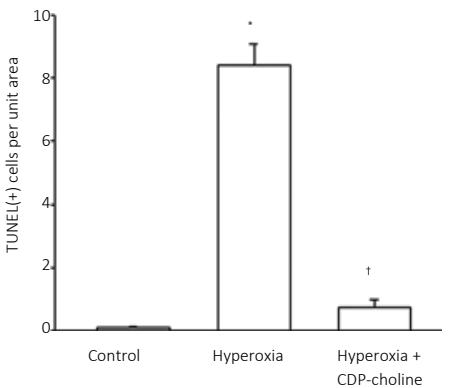

Fig. 3. TUNEL-positive cell counts. (a) Immunohistochemistry images and (b) bar graph for TUNEL-positive cell counts. Arrows indicate TUNEL-positive cells. ${ }^{*} P<0.05$, significantly different from control group; $+P<0.05$, significantly different from hyperoxia group using one-way ANOVA followed by post hoc Holm-Sidak test. Bar, $25 \mu \mathrm{m}$. CDP-choline, cytidine 5'-diphosphocholine; TUNEL, terminal deoxynucleotidyl transferase-mediated deoxyuridine triphosphate nick-end labeling.

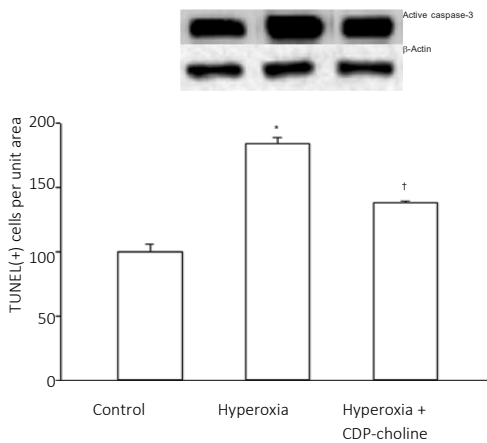

Fig. 4. Bar graph showing the effect of CDP-choline treatment on active caspase-3 expression in hyperoxic lung injury. ${ }^{*} P<0.05$, significantly different from control group; $+P<0.05$, significantly different from hyperoxia group using one-way ANOVA followed by post hoc Holm-Sidak test. $\beta$-Actin was used as a protein-loading control. CDP-choline, cytidine 5'-diphosphocholine. 


\section{Chapter 3}

Table 1. Levels of cytokines in lung homogenates

\begin{tabular}{llll}
\hline & & & Hyperoxia + \\
Cytokine levels, $\mathrm{pg} / \mathrm{ml}$ & Control group & Hyperoxia group & CDP-choline group \\
\hline IL-1 $\beta$ & $91.2 \pm 8.7$ & $148.9 \pm 12.4^{* *}$ & $112.1 \pm 9.4^{* * *}$ \\
IL-6 & $24.4 \pm 3.2$ & $43.7 \pm 4.5^{*}$ & $28.6 \pm 4.1^{* * *}$ \\
TNF- $\alpha$ & $104.0 \pm 20.3$ & $246.7 \pm 30.9^{* *}$ & $146.2 \pm 20.7^{* * *}$ \\
\hline
\end{tabular}

CDP-choline, cytidine 5'-diphosphocholine; IL, interleukin; TNF, tumor necrosis factor.

${ }^{*} P<0.05 ;{ }^{* * P}<0.01$ compared with control group; ***P $<0.05$ compared with hyperoxia group using one-way ANOVA followed by post hoc Holm-Sidak test.

Table 2. Lung tissue and bronchoalveolar lavage fluid phospholipid levels in the study groups

\begin{tabular}{|c|c|c|c|}
\hline & Control group & Hyperoxia group & $\begin{array}{l}\text { Hyperoxia }+ \\
\text { CDP-choline group }\end{array}$ \\
\hline \multicolumn{4}{|c|}{ Lung tissue phospholipids (nmol/mg protein) } \\
\hline Total phospholipids & $298.5 \pm 6.4$ & $220.8 \pm 5.4^{* *}$ & $271.8 \pm 1.5^{+}$ \\
\hline $\mathrm{PC}$ & $146 \pm 2.7$ & $107.5 \pm 3.2^{* *}$ & $130 \pm 1^{+}$ \\
\hline DSPC & $47.5 \pm 1.2$ & $35.5 \pm 1 * *$ & $42.5 \pm 1^{+}$ \\
\hline PG & $9.5 \pm 0.2$ & $7.1 \pm 0.2^{*}$ & $8.1 \pm 0.2^{* * *}$ \\
\hline \multicolumn{4}{|c|}{ BAL fluid phospholipids (nmol/ml) } \\
\hline Total phospholipids & $31 \pm 1.6$ & $20.5 \pm 0.6^{* *}$ & $28.5 \pm 0.7^{+}$ \\
\hline $\mathrm{PC}$ & $24.3 \pm 0.5$ & $17.9 \pm 0.5^{* *}$ & $21.7 \pm 0.2^{+}$ \\
\hline DSPC & $15.8 \pm 0.4$ & $11.8 \pm 0.3^{* *}$ & $14.2 \pm 0.1$ \\
\hline$P G$ & $3.2 \pm 0.1$ & $2.4 \pm 0.1^{*}$ & $2.7 \pm 0.1^{* * *}$ \\
\hline
\end{tabular}

BAL, bronchoalveolar lavage; DSPC, disaturated phosphatidylcholine; PC, phosphatidylcholine; PG, phosphatidylglycerol.

$* P<0.05 ; * *<0.01$ compared with control group; ${ }^{* * *} P<0.05 ;+P<0.01$ compared with hyperoxia group using one-way ANOVA followed by post hoc Holm-Sidak test.

Table 3. Lung tissue malondialdehyde (MDA) content and superoxide dismutase (SOD), glutathione peroxidase (GSH-Px), and myeloperoxidase (MPO) activities

\begin{tabular}{llll}
\hline & $\begin{array}{l}\text { Control } \\
\text { group, } \mathrm{n}=10\end{array}$ & $\begin{array}{l}\text { Hyperoxia } \\
\text { group, } \mathrm{n}=10\end{array}$ & $\begin{array}{l}\text { Hyperoxia }+ \\
\text { CDP-choline group } \\
\mathrm{n}=10\end{array}$ \\
\hline Biochemical analyses & & & \\
MDA (nmol/g protein) & $31.1 \pm 3.7$ & $68.8 \pm 7.4^{* *}$ & $52.9 \pm 3.2^{* * *}$ \\
SOD (U/mg protein) & $114.4 \pm 9.2$ & $63.7 \pm 2.5^{* *}$ & $95.6 \pm 6.1^{+}$ \\
GSH-Px (U/mg protein) & $12.5 \pm 1.4$ & $5.6 \pm 1.8^{*}$ & $11.5 \pm 1.5^{* * *}$ \\
MPO (U/g protein) & $36.5 \pm 2.3$ & $76.2 \pm 2.1^{* *}$ & $55.5 \pm 6.8^{\dagger}$ \\
\hline
\end{tabular}

CDP-choline, cytidine $5^{\prime}$-diphosphocholine.

$* P<0.05 ; * * P<0.01$ compared with control group; ${ }^{* * *} P<0.05 ;+P<0.01$ compared with hyperoxia group using one-way ANOVA followed by post hoc Holm-Sidak test. 


\section{DISCUSSION}

These data show that CDP-choline treatment improves histopathological score, decreases fibrosis, improves alveolarization by preserving radial alveolar count and lamellar body protein expression in the alveoli, reduces TUNEL-positive cell counts and active caspase-3 expression, increases antioxidant activity, and decreases lipid peroxidation and inflammation in the injured lung tissues of neonatal rats. In addition, both lung tissue and BAL fluid phospholipid contents were significantly increased by CDPcholine therapy. To the best of our knowledge, this is the first experimental study that reports amelioration of hyperoxic lung injury by CDP-choline treatment in a neonatal rat model.

Preterm infants and their lungs are susceptible to injury as a result of resuscitation, mechanical ventilation, oxygen therapy, and surfactant deficiency (10). BPD is characterized by decreased alveolar and vascular lung development resulting in enlarged alveoli and is complicated by inflammation, abnormal coagulation, fibrinolysis, oxidative stress, and, at later stages, pulmonary hypertension (10). Neonatal exposure to hyperoxia in animals was reported to result in progressive lung disease that strongly resembles BPD in premature infants (11). Therefore, we used this experimental BPD model, which induces lung injury by exposure to hyperoxia.

Hyperoxic injury caused both apoptotic and necrotic cell death in an adult murine model, and the number of apoptotic cells in the lung exposed to hyperoxia correlated with the degree of the injury (12). High concentrations of oxygen also resulted in apoptosis in the lungs in a neonatal mouse model in which the degree of apoptosis was found to be associated with the duration of hyperoxia and the degree of lung injury (13). Therefore, the authors suggested that apoptosis due to hyperoxia during the crucial period of lung development might adversely affect future lung growth. In accordance with these studies, our data showed that CDP-choline significantly decreased the number of TUNEL-positive cells and active caspase-3 expression in hyperoxic lung injury.

Although BPD has a multifactorial etiology, pre- and postnatal infection and inflammation are the major contributors to its pathogenesis (2). Several studies showed increased protein levels and high mRNA expression of proinflammatory cytokines in airway secretions and bronchoalveolar cells of premature infants who developed BPD $(14,15)$. We also observed in the current study that proinflammatory cytokines TNF- $\alpha$, IL6 , and IL-1 $\beta$ were increased due to exposure to hyperoxia in the lungs of neonatal rats. However, CDP-choline treatment significantly reduced the levels of these proinflammatory cytokines. CDP-choline is a choline donor because part of exogenously administered CDP-choline is metabolized to choline in the circulation (16). Choline is an essential nutrient and a precursor of the neurotransmitter acetylcholine (ACh) as well as various membrane phospholipids (17). Choline may have anti-inflammatory effects by activating $\alpha-7$ nicotinic ACh receptors (18). In agreement, choline administration was shown to reduce the elevation of circulating TNF- $\alpha$ levels and attenuate endotoxin- 
induced multiple organ injury (19), whereas choline deficiency results in increased levels of proinflammatory cytokines such as TNF- $\alpha$ (20). Because exogenously administered CDP-choline is a source of choline (16), our observation that CDP-choline reduces tissue levels of proinflammatory cytokines might have resulted from the anti-inflammatory action of choline (through stimulation of $\alpha-7$ nicotinic ACh receptors) that derived from CDP-choline metabolism.

In addition, because choline is a precursor for the synthesis of ACh both in the brain and periphery, including nonneuronal cells in the respiratory tract (21), choline metabolized from CDP-choline might have enhanced ACh synthesis and cholinergic activity in our study. Recent findings suggest that through the cholinergic antiinflammatory pathway (22), ACh regulates additional functions in the airways, including inflammation and remodeling during inflammatory airway diseases $(23,24)$. Therefore, because CDP-choline administration has been shown to exhibit body responses of a cholinergic nature (25), the anti-inflammatory effects of CDP-choline might have resulted from enhanced ACh synthesis and cholinergic activity. Another mechanism by which CDPcholine prevented BPD in our study might include counteraction of cytokine effects on cellular membranes by CDP-choline. Exogenously administered CDP-choline enhances membrane phospholipid levels in the brain and lungs (8). Therefore, as a building block of PC, the major constituent of surfactant, CDP-choline might have shown benefit by enhancing surfactant synthesis in our study. Surfactant therapy was suggested to confer benefit in the prevention of BPD in previous studies that demonstrated persisting surfactant abnormalities including an ongoing quantitative deficiency in surfactant components (especially surfactant proteins), decreased function of endogenous surfactant for lowering surface tension, and increased surfactant turnover in ventilated preterms evolving BPD (6). In addition, expression of surfactant proteins A, B, and C were found to be altered in animal models of chronic exposure to high oxygen concentrations (26). The relative deficiencies of surfactant proteins were reported to be indicators of BPD for lower lung volume and infection in evolving BPD in a baboon model of BPD (27). In another study, surfactant function and contents of surfactant proteins were shown to be altered in mechanically ventilated preterm infants (6). In accordance with these data, temporary improvement in pulmonary function of preterm infants who required prolonged mechanical ventilation was achieved with exogenous surfactant supplementation (28), suggesting surfactant's preventive effect on BPD development (7). Hence, we analyzed both lung tissue and BAL fluid phospholipid concentrations and showed that the phospholipid classes PC, phosphatidylglycerol, and DSPC reached nearcontrol levels after CDP-choline administration. Therefore, as a precursor of PC and surfactant synthesis, CDP-choline might have ameliorated the lung damage caused by BPD in our study by reducing membrane breakdown and enhancing phospholipid and, possibly, surfactant production.

Oxygen toxicity, which has been suggested as a significant contributing factor for BPD development in premature infants (2), is associated with generation of hydroxyl radicals 
and peroxidation of membrane lipids. It is well known that proinflammatory cytokines activate phospholipase $A_{2}$ and PC-phospholipase $C$ in order to hydrolize PC (4). PC hydrolysis by phospholipase A2 results in the release of lyso-PC, an inhibitor of CTPphosphocholine cytidylyltransferase enzyme, which is involved in PC synthesis, and free fatty acids including arachidonic acid, which is a significant source of reactive oxygen species. CDP-choline was previously reported to prevent membrane destruction, decrease free radical generation, and preserve the natural mechanisms against oxidative stress, particularly in hypoxia-ischemia models (4). In agreement with these observations, our data indicate that CDP-choline treatment preserves GSH-Px and SOD activities as well as reducing tissue MDA content and MPO activity, suggesting that one of the mechanisms by which CDP-choline decreases hyperoxic lung injury is enhancement of antioxidant activity and reduction of lipid peroxidation in a neonatal rat model of BPD. It may also suggest that the effect of CDP-choline on apoptosis might have resulted from attenuation of oxidative stress by restoration of antioxidant enzyme levels.

A number of previous studies evaluated surfactant kinetics in preterm infants who did or did not develop BPD. One study reported that tracheal aspirate DSPC concentration decreased significantly in infants who subsequently developed BPD (29). The loss of DSPC palmitate from the alveolar pool was significantly higher, whereas recycling of DSPC palmitate through the intracellular pool was significantly lower (30). Therefore, our findings regarding the increased BAL fluid and lung tissue phospholipid contents after CDP-choline administration might explain the possible role of CDP-choline in the attenuation of hyperoxic lung injury in our experimental setting.

To the best of our knowledge, two previous human studies evaluated CDP-choline treatment in preterm infants with respiratory distress syndrome $(8,9)$. Although parenteral CDP-choline administration showed no beneficial effects at a $100 \mathrm{mg} / \mathrm{kg}$ dosage for $7 \mathrm{~d}$ (9), it was reported to reduce the severity of respiratory distress and oxygen requirement when it was administered at a $300 \mathrm{mg} / \mathrm{kg}$ dosage for a longer duration (8). Therefore, a higher dose and a longer duration of CDP-choline administration might explain the beneficial effect of CDP-choline against hyperoxic lung injury in our study and suggests its applicability in newborns with BPD.

In conclusion, our study is the first to show the efficacy of CDP-choline treatment in hyperoxic lung injury in a neonatal rat model by several mechanisms including enhancement of phospholipid production as well as reduction of fibrosis, apoptosis, and lipid peroxidation. These findings might provide new insights into the prevention of BPD development by CDP-choline treatment in premature infants. Nevertheless, our study is limited by the lack of dose and time courses of CDP-choline effects, and future studies are warranted to further confirm its effects and reveal the mechanism of action of CDPcholine treatment in hyperoxic lung injury and BPD. 


\section{METHODS}

\section{Animals and Experimental Design}

This study was approved by the Experimental Animal Ethics Committee of Gulhane Military Medical Academy (Ankara, Turkey). In addition, the guidelines provided by the US National Institutes of Health (Bethesda, MD) for the care and use of laboratory animals were followed.

Sprague Dawley rats with dated pregnancies were housed in individual cages with free access to water and laboratory chow. Pups born by spontaneous delivery to four timemated full-term Sprague Dawley pregnant rats were pooled, randomized, and delivered back to nursing dams. A total of 30 newborn pups were divided equally into three groups as follows: control group (composed of pups subjected to room air containing $21 \% \mathrm{O}_{2}$ and receiving saline), hyperoxia group (composed of pups subjected to $95 \% \mathrm{O}_{2}$ and receiving saline), and hyperoxia + CDP-choline group (composed of pups subjected to 95\% $\mathrm{O}_{2}$ and receiving $300 \mathrm{mg} / \mathrm{kg} / \mathrm{d}$ CDP-choline).

Experiments began immediately after birth and continued throughout P10. Nursing dams were rotated between hyperoxia-exposed and room air-exposed litters every $24 \mathrm{~h}$ to prevent oxygen toxicity in the dams. In pups subjected to hyperoxia, continuous $95 \%$ $\mathrm{O}_{2}$ exposure was achieved in a Plexiglas chamber $(70 \times 60 \times 30 \mathrm{~cm})$ by a flow-through system. The oxygen level inside the Plexiglas chamber was monitored continuously with a Ceramatec oxygen analyzer (MAXO2; Ceramatec, Salt Lake City, UT) to maintain $\geq 95 \% \mathrm{O}_{2}$ saturation. $\mathrm{CO}_{2}$ was kept below $0.5 \%$ using a gas monitor (Apex; BW Technologies, Lincolnshire, IL). Temperature and humidity were maintained at $22-25^{\circ} \mathrm{C}$ and $60-70 \%$, respectively.

Intraperitoneal injections of saline $(4 \mathrm{ml} / \mathrm{kg})$ in the control and hyperoxia groups and those of CDP-choline $(300 \mathrm{mg} / \mathrm{kg})$ in the hyperoxia + CDP-choline group were performed daily from the first day of life (P1) through P10, when the study was terminated. The pups in each group were weighed daily using a sensitive scale of $0.01 \mathrm{~g}$, and their weights were recorded.

\section{Lung Tissue Procurement}

Pups were killed at P10 under deep anesthesia by intraperitoneal injection of a combination of ketamine (100 mg/kg, Ketalar; Zentiva Saglik Urunleri, Kirklareli, Turkey) and xylazine (10 mg/kg, Xylazine Bio 2\%; Bioveta, Ivanovice na Hane, Czech Republic). The thorax was opened and the lungs were exposed. Right lungs of rat pups were excised and weighed immediately for analyses of tissue cytokine levels, active caspase-3 expression, phospholipid content, and oxidant/antioxidant enzyme activities and MDA content, whereas left lungs were obtained after ligation of the right main bronchus and perfusion with a fixation solution for histopathologic and immunohistochemical evaluation. 


\section{Histopathologic and Immunohistochemical Evaluation of the Lungs}

Left lungs were perfused with $0.1 \mathrm{~mol} / \mathrm{l}$ phosphate-buffered saline (PBS) containing $4 \%$ paraformaldehyde under a constant inflation pressure of $5 \mathrm{~cm} \mathrm{H} \mathrm{H}_{2} \mathrm{O}$ maintained via a tracheal catheter. On completion of perfusion, the trachea was ligated with a surgical suture, and the lungs were incubated in fresh $4 \%$ paraformaldehyde-PBS solution on ice for 4-5 h. The perfusion solution was then replaced with two quick changes of cold PBS to remove exterior debris. The lungs were transferred to a filtered sterile PBS $/ 30 \%$ sucrose solution and stored at $4^{\circ} \mathrm{C}$ until fully equilibrated. The lungs were then paraffin embedded, and these paraffin blocks were sliced into $5-\mu m$ sections. Sections were chosen according to a systematic random sampling procedure and then mounted onto poly-I-lysine-coated slides (Histobond adhesion slides; Paul Marienfeld, LaudaKonigshofen, Germany).

Slides were stained with standard hematoxylin-eosin and Masson's trichrome techniques for histopathologic evaluations and with $A B C$ technique for the lamellar body membrane protein. For immunohistochemical detection of lamellar body membrane protein, sections were subjected to dehydration following treatment with 3\% hydrogen peroxide for $30 \mathrm{~min}$. After nonspecific blocking with goat serum for $30 \mathrm{~min}$, the sections were incubated overnight with primary antibody against P180 lamellar body protein (Abcam, Cambridge, MA) at $4^{\circ} \mathrm{C}$, followed by treatment with biotinylated antimouse secondary anti- body (1:200; Vector Laboratories, Peterborough, UK) for $30 \mathrm{~min}$ at room temperature. Following the avidin-biotin complex treatment, 3,3'-diaminobenzidine (Vector Laboratories) was used for color development. Negative control slides, omitting the primary antibody, were also included. The tissue sections were examined using a light microscope equipped with a digital camera. Positive cells per unit area were counted five times on $25 \mu \mathrm{m}$ apart sections, 10 sections for each animal. Histopathologic examination and immunohistochemical scoring were performed in a blinded manner by the same investigator. Histopathologic grading was done as follows: grade 1, normal histology; grade 2, moderate leukocytic infiltration; grade 3, leukocytic infiltration, edema, and partial destruction; and grade 4, total destruction of the tissue as described previously (31). Radial alveolar count for the assessment of alveolar development was performed on the digital images of sections. Briefly, a line was superimposed from a center of a respiratory bronchiole to the nearest connective tissue septum at right angles to the epithelium, and the number of alveolar septi crossed by this line was counted on 3-4 sections for each animal (32). Apoptosis was evaluated by TUNEL technique using an in situ cell death detection POD kit (Roche Molecular Biochemicals, Mannheim, Germany). Sections were chosen according to a systematic random sampling procedure and then mounted onto poly-l-lysine-coated slides (Histobond adhesion slides; Paul Marienfeld). Sections were heated at $60^{\circ} \mathrm{C}$ for $45 \mathrm{~min}$, followed by washing in xylene and rehydration through a graded series of alcohol (ethanol 100, 95, 80, and 70\%), and washed in distilled water. They were then incubated in $20 \mu \mathrm{g} / \mathrm{ml}$ proteinase $\mathrm{K}$ solution (Roche Applied Sciences, Penzberg, Germany) for $20 \mathrm{~min}$ at room temperature and 
immersed in $3 \% \mathrm{H}_{2} \mathrm{O}_{2}$ in methanol for 10 min. Permeabilization with Triton X (Sigma-Aldrich, St Louis, MO) for 10 min was performed on ice. The sections were then incubated with TUNEL reaction mixture at $37^{\circ} \mathrm{C}$ in a humidified chamber for $1 \mathrm{~h}$ followed by converterperoxidase treatment at $37^{\circ} \mathrm{C}$ for $30 \mathrm{~min}$. Diaminobenzidine tetrahydrochloride (diaminobenzidine/metal concentrate: peroxidase buffer; Roche Applied Sciences) was used as the chromogene, and the slides were counterstained with hematoxylin (Harris's hematoxylin; Sigma-Aldrich) and coverslipped (DPX mountant; Fluka, St Louis, MO) for light microscopy.

\section{Active Caspase-3 Expression}

Right lungs were homogenized by 50-fold dilution (wt/vol) in ice-cold PBS ( $\mathrm{pH} 7.2$ ) using a homogenizer (T18 basic Ultra-Turrax; IKA, Staufen, Germany). Aliquots of homogenates were used for total protein analysis by bicinchoninic acid assay (Thermo Fisher Scientific, Rockford, IL). Aliquots of homogenates were mixed with equal volumes of Laemmi loading buffer and boiled for 5 min before gel electrophoresis. Equal amounts of protein were loaded and separated using sodium dodecyl sulfate-polyacrylamide gel electrophoresis (4-20\%; Bio-Rad, Hercules, CA). Proteins were then transferred onto polyvinylidene fluoride membranes (Immobilon-P; Millipore, Billerica, MA). The remaining binding sites were blocked with $4 \%$ nonfat dry milk (Carnation, Glendale, CA) for 30 min in Tris-buffered saline with Tween (TBST). Membranes were then rinsed five times in TBST buffer and incubated overnight in TBST solution containing the primary antibody against active caspase-3 (Abcam) and $\beta$-actin (Abcam), which was used as a loading control for lung homogenates. Following over-night incubation and five rinses in TBST buffer, blots were incubated for $1 \mathrm{~h}$ with the appropriate peroxidase-linked secondary antibody (GE Healthcare, Waukesha, WI). Blots were then rinsed in TBST buffer five times, and proteinantibody complexes were detected and visualized using the enhanced chemiluminescence system (GE Healthcare) and developed on Kodak X-AR film (Kodak Turkiye, Istanbul, Turkey). Films were digitized using a Supervista S-12 scanner with a transparency adapter (UMAX Technologies, Freemont, CA). Immunoreactive bands were compared densitometrically using the public domain NIH Image program available on the Internet at http://rsbweb.nih.gov/ij/download.html. Areas under the absorbance curve were expressed as arbitrary units and normalized as percentages of those generated in the same blot using samples from lungs of control animals.

\section{Measurement of Lung Tissue Cytokine Content}

Aliquots of homogenates were centrifuged at $12,000 \mathrm{~g}$ for $20 \mathrm{~min}$ at $4^{\circ} \mathrm{C}$, and cell-free supernatants were used for determining lung tissue proinflammatory cytokine (TNF- $\alpha$, IL6 , and IL-1 $\beta$ ) contents by specific enzyme-linked immunosorbent assay kits (R\&D Systems, Minneapolis, MN) as described previously (33). 


\section{BAL Fluid Collection}

In separate experiments, rat pups anesthetized with intraperitoneal ketamine plus xylazine were placed in a supine position, and a cannula was inserted into the trachea. Ice-cold isotonic saline $(1 \mathrm{ml})$ was gently instilled with a syringe into the lung via the trachea and then withdrawn 10 times. Recovered BAL fluid was immediately centrifuged at 5,000 rpm for $10 \mathrm{~min}$ to remove cells, and samples from four pups from each treatment group were pooled.

\section{Phospholipid Assay}

Phospholipids were extracted from lung tissue homogenates and BAL fluid using a chloroform-methanol-water mixture (2:1:1 vol/vol/vol) as described previously (34). These extracts were used for analyzing total phospholipid content and individual phospholipids (PC, phosphatidylglycerol, and DSPC). Total phospholipid content was analyzed by measuring phosphorus (35). Part of the remaining extracts was subjected to thin-layer chromatography using silica G plates (Adsorbosil Plus-1; Alltech, Deerfield, IL) and a system consisting of chloroform/ethanol/triethylamine/water (30:34:30:8) as the mobile phase to analyze PC and phosphatidylglycerol, whereas the other part was oxidized with osmium tetroxide $\left(\mathrm{OSO}_{4}\right)$ before subjection to thin-layer chromatography to analyze DSPC, the major surfactant component, as described previously (36). The chromatography plates were sprayed with $0.1 \%$ diphenylhexatriene in petroleum ether, and phospholipid standards were used to identify the corresponding bands, which were scraped off the plates and extracted into methanol, dried under a vacuum, and assayed for phosphorus content as described previously (35).

\section{Analyses}

Activities of SOD, GSH-Px, and MPO, as well as MDA content of lung tissues, were measured using cell-free supernatants of right-lung homogenates by spectrophotometric (UV-1700; Shimadzu, Kyoto, Japan) analyses.

Tissue SOD activity was determined by a method involving spectrophotometric detection of formazan production at $550 \mathrm{~nm}$ by inhibition of nitroblue tetrazolium reduction with xanthine-xanthine oxidase used as a superoxide generator as described previously (37). Tissue GSH-Px activity was determined by the dithio-dinitrobenzoic acid method at the absorbance of $412 \mathrm{~nm}$ as described previously (38). Tissue MDA content was determined by a method that is based on the reaction of MDA with thiobarbituric acid at $95^{\circ} \mathrm{C}$ as described previously (39). Tissue MPO activity was determined by a method based on the reduction of 5-thio-2-nitrobenzoic acid to 5-5'-dithiobis (2nitrobenzoic acid) by following the decrease of absorbance at $412 \mathrm{~nm}$ as described previously (40). 


\section{Statistical Analyses}

Statistical analyses were performed using SPSS 16.0 (SPSS, Chicago, IL). A level of P $<0.05$ was chosen to represent statistical significance. Experimental group means and SEs were determined using descriptive statistics, and data were expressed as mean \pm SEM. Normal distributions of data were graphically examined with the Shapiro-Wilk test. Categorical variables were compared with the $x^{2}$ test. Immunohistochemical scores were assessed by the Kruskal-Wallis test. Treatment groups were compared using one-way ANOVA, and significance was determined using a post hoc Holm-Sidak test or Bonferroni's correction for multiple comparisons where applicable.

\section{STATEMENT OF FINANCIAL SUPPORT}

No financial assistance was received to support this work. Disclosure: The authors declare no conflict of interest. 


\section{REFERENCES}

1. Thomas W, Speer CP. Prevention and treatment of bronchopulmonary dysplasia: current status and future prospects. J Perinatol 2007;27: S26-32.

2. Gien J, Kinsella JP. Pathogenesis and treatment of bronchopulmonary dysplasia. Curr Opin Pediatr 2011;23:305-13.

3. Laughon MM, Smith PB, Bose C. Prevention of bronchopulmonary dysplasia. Semin Fetal Neonatal Med 2009;14:374-82.

4. Adibhatla RM, Hatcher JF. Cytidine 5'-diphosphocholine (CDP-choline) in stroke and other CNS disorders. Neurochem Res 2005;30: 15-23.

5. Zimmermann LJ, Janssen DJ, Tibboel D, Hamvas A, Carnielli VP. Surfac- tant metabolism in the neonate. Biol Neonate 2005;87:296-307.

6. Merrill JD, Ballard RA, Cnaan A, et al. Dysfunction of pulmonary surfac- tant in chronically ventilated premature infants. Pediatr Res 2004;56:918- 26.

7. Laughon M, Bose C, Moya F, et al.; Surfaxin Study Group. A pilot randomized, controlled trial of later treatment with a peptide-containing, synthetic surfactant for the prevention of bronchopulmonary dysplasia. Pediatrics 2009;123:89-96.

8. Colombo ML, Dogliani P, Raggi M. [Relation between citicoline and pulmonary surfactant. Recent acquisitions in the treatment of respiratory syndromes of the newborn]. Minerva Pediatr 1976;28: 2303-10.

9. Valls i Soler A, Sanjurjo P, Vazquez Cordero C. [Controlled study of the administration of CDP-choline to preterm newborn infants with respira- tory distress syndrome]. An Esp Pediatr 1988;28:493-6.

10. Jobe $A H$, Ikegami M. Mechanisms initiating lung injury in the preterm. Early Hum Dev 1998;53:81-94.

11. Warner BB, Stuart LA, Papes RA, Wispé JR. Functional and pathological effects of prolonged hyperoxia in neonatal mice. Am J Physiol 1998;275(1 Pt 1):L110-7.

12. Mantell LL, Horowitz S, Davis JM, Kazzaz JA. Hyperoxia-induced cell death in the lung-the correlation of apoptosis, necrosis, and inflamma- tion. Ann NY Acad Sci 1999;887:171-80.

13. McGrath-Morrow SA, Stahl J. Apoptosis in neonatal murine lung exposed to hyperoxia. Am J Respir Cell Mol Biol 2001;25:150-5.

14. Viscardi RM, Muhumuza CK, Rodriguez A, et al. Inflammatory markers in intrauterine and fetal blood and cerebrospinal fluid compartments are associated with adverse pulmonary and neurologic outcomes in preterm infants. Pediatr Res 2004;55:1009-17.

15. Paananen R, Husa AK, Vuolteenaho R, Herva R, Kaukola T, Hallman M. Blood cytokines during the perinatal period in very preterm infants: rela- tionship of inflammatory response and bronchopulmonary dysplasia. J Pediatr 2009;154:39-43.e3.

16. Cansev M, Yilmaz MS, Ilcol YO, Hamurtekin E, Ulus IH. Cardiovascular effects of CDP-choline and its metabolites: involvement of peripheral auto- nomic nervous system. Eur J Pharmacol 2007;577:129-42.

17. Wurtman RJ, Cansev M, Ulus IH. Choline and its products acetylcho- line and phosphatidylcholine. In: Lajtha A, ed. Neural Lipids, Handbook of Neurochemistry and Molecular Neurobiology. Berlin, Heidelberg: Springer-Verlag, 2009:443-501.

18. Parrish WR, Rosas-Ballina M, Gallowitsch-Puerta M, et al. Modulation of TNF release by choline requires alpha7 subunit nicotinic acetylcholine receptor-mediated signaling. Mol Med 2008;14:567-74.

19. Ilcol YO, Yilmaz Z, Ulus IH. Endotoxin alters serum-free choline and phospholipid-bound choline concentrations, and choline administration attenuates endotoxin-induced organ injury in dogs. Shock 2005;24:288-93.

20. Holmes-McNary MQ, Baldwin AS Jr, Zeisel SH. Opposing regulation of choline deficiency-induced apoptosis by p53 and nuclear factor kappaB. J Biol Chem 2001;276:41197-204.

21. Kolahian S, Gosens R. Cholinergic regulation of airway inflammation and remodelling. J Allergy (Cairo) 2012;2012:681258.

22. Tracey KJ. Physiology and immunology of the cholinergic antiinflamma- tory pathway. J Clin Invest 2007;117:289-96. 
23. de Jonge WJ, Ulloa L. Th alpha7 nicotinic acetylcholine receptor as a pharmacological target for infl mmation. Br J Pharmacol 2007;151: 915-29.

24. Blanchet MR, Israël-Assayag E, Cormier Y. Modulation of airway inflam- mation and resistance in mice by a nicotinic receptor agonist. Eur Respir J 2005;26:21-7.

25. Cansev M, Ilcol YO, Yilmaz MS, Hamurtekin E, Ulus IH. Choline, CDP- choline or phosphocholine increases plasma glucagon in rats: involve- ment of the peripheral autonomic nervous system. Eur J Pharmacol 2008;589:315-22.

26. Savani RC, Godinez RI, Godinez MH, et al. Respiratory distress after intra- tracheal bleomycin: selective deficiency of surfactant proteins B and C. Am J Physiol Lung Cell Mol Physiol 2001;281:L685-96.

27. Ballard PL, Gonzales LW, Godinez RI, et al. Surfactant composition and function in a primate model of infant chronic lung disease: effects of inhaled nitric oxide. Pediatr Res 2006;59:157-62.

28. Katz LA, Klein JM. Repeat surfactant therapy for postsurfactant slump. J Perinatol 2006;26:414-22.

29. Cogo PE, Zimmermann $\sqcup$, Pesavento $R$, et al. Surfactant kinetics in pre- term infants on mechanical ventilation who did and did not develop bron- chopulmonary dysplasia. Crit Care Med 2003;31:1532-8.

30. Cogo PE, Toffolo GM, Gucciardi A, Benetazzo A, Cobelli C, Carnielli VP. Surfactant disaturated phosphatidylcholine kinetics in infants with bronchopulmonary dysplasia measured with stable isotopes and a two- compartment model. J Appl Physiol 2005;99:323-9.

31. Ozdulger A, Cinel I, Koksel $\mathrm{O}$, et al. The protective effect of $\mathrm{N}$-acetylcyste- ine on apoptotic lung injury in cecal ligation and puncture-induced sepsis model. Shock 2003;19:366-72.

32. Askenazi SS, Perlman M. Pulmonary hypoplasia: lung weight and radial alveolar count as criteria of diagnosis. Arch Dis Child 1979;54:614-8.

33. Bouadma L, Schortgen F, Ricard JD, Martet G, Dreyfuss D, Saumon G. Ventilation strategy affects cytokine release after mesenteric ischemia- reperfusion in rats. Crit Care Med 2004;32:1563-9.

34. Folch J, Lees M, Sloane Stanley GH. A simple method for the isolation and purification of total lipides from animal tissues. J Biol Chem 1957;226:497- 509.

35. Svanborg A, Svennerholm L. Plasma total lipids, cholesterol, triglycerides, phospholipids and free fatty acids in a healthy Scandinavian population. Acta Med Scand 1961;169: 43-9.

36. Mason RJ, Nellenbogen J, Clements JA. Isolation of disaturated phosphati- dylcholine with osmium tetroxide. J Lipid Res 1976;17:281-4.

37. Sun Y, Oberley LW, Li Y. A simple method for clinical assay of superoxide dismutase. Clin Chem 1988;34:497-500.

38. Paglia DE, Valentine WN. Studies on the quantitative and qualitative characterization of erythrocyte glutathione peroxidase. J Lab Clin Med 1967;70:158-69.

39. Draper $\mathrm{HH}$, Hadley M. Malondialdehyde determination as index of lipid peroxidation. Meth Enzymol 1990;186:421-31.

40. Weiss SJ, Klein R, Slivka A, Wei M. Chlorination of taurine by human neutrophils. Evidence for hypochlorous acid generation. J Clin Invest 1982;70:598-607. 


\section{Chapter}

\section{CDP-choline reduces severity of intestinal injury in a neonatal rat model of necrotizing enterocolitis}

Published as: Cetinkaya M, Cansev M, Cekmez F, Tayman C, Canpolat FE, Kafa IM, Uysal $S$, Tunc T, Sarici SU. CDP-choline reduces severity of intestinal injury in a neonatal rat model of necrotizing enterocolitis. J Surg Res 2013; 183: 119-128. 


\section{Abstract}

\section{Background:}

Cytidine 5'-diphosphocholine (CDP-choline) is an endogenous intermediate in the biosynthesis of phosphatidylcholine, a contributor to the mucosal defense of the intestine. The aim of this study was to evaluate the possible cytoprotective effect of CDPcholine treatment on intestinal cell damage, membrane phospholipid content, inflammation, and apoptosis in a neonatal rat model of necrotizing enterocolitis (NEC).

\section{Methods:}

We divided a total of 30 newborn pups into three groups: control, NEC, and NEC + CDPcholine. We induced NEC by enteral formula feeding, exposure to hypoxia- hyperoxia, and cold stress. We administered CDP-choline intraperitoneally at $300 \mathrm{mg} / \mathrm{kg} / \mathrm{d}$ for $3 \mathrm{~d}$ starting from the first day of life. We evaluated apoptosis macroscopically and histopathologically in combination with proinflammatory cytokines in the gut samples. Moreover, we determined membrane phospholipid levels as well as activities of xanthine oxidase, superoxide dismutase, glutathione peroxidase, and myeloperoxidase enzymes and the malondialdehyde content of intestinal tissue.

\section{Results:}

Mean clinical sickness score, macroscopic gut assessment score, and intestinal injury score were significantly improved, whereas mean apoptosis score and caspase-3 levels were significantly reduced in pups in the NEC + CDP-choline group compared with the NEC group. Tissue proinflammatory cytokine (interleukin- $1 \beta$, interleukin-6, and tumor necrosis factor- $\alpha$ ) levels as well as tissue malondialdehyde content and myeloperoxidase activities were reduced, whereas glutathione peroxidase and superoxide dismutase activities were preserved in the NEC + CDP-choline group. In addition, NEC damage reduced intestinal tissue membrane phospholipids, whereas CDP-choline significantly enhanced total phospholipid and phosphatidylcholine levels. Long-term follow-up in additional experiments revealed increased body weight, decreased clinical sickness scores, and enhanced survival in CDP-choline-receiving versus saline-receiving pups with NEC lesions.

\section{Conclusions:}

Our study reports, for the first time, beneficial effects of CDP-choline treatment on intestinal injury in a neonatal rat model of NEC. Our data suggest that CDP-choline may be used as an effective therapeutic agent to prevent NEC. 


\section{Introduction}

Necrotizing enterocolitis (NEC) is the most common and potentially most fatal gastrointestinal (GI) disease in neonates [1]. It remains a leading cause of mortality, with rates ranging from $10 \%$ to $50 \%$ [2]. The pathogenesis of NEC is likely multifactorial and is poorly understood. To date, prematurity, intestinal immaturity, hypoxia-ischemia, low perfusion states, formula feeding, and colonization with pathogenic bacteria were reported to lead the common pathway of bowel injury, intestinal inflammation, and release of proinflammatory cytokines, resulting in intestinal tissue damage including necrosis and apoptosis in intestinal tissue [3]. In addition, immature motility, digestion, absorption, immune defenses, barrier function, and circulatory regulation were reported to increase the risk of intestinal injury [4].

Based on clinical and experimental data, all risk factors initiate the activation of proinflammatory response, which leads to bowel necrosis and, in advanced cases, multiorgan dysfunction syndrome and death [5]. Previous studies showed an intestinal mucosal injury mediated by oxygen-derived free radicals, inflammatory mediators including platelet-activating factor, leukotrienes, and tumor necrosis factor-alpha (TNF$\alpha$ ). As a result, an initial stress might trigger the release of platelet-activating factor, subsequent release of inflammatory cytokines, and migration of activated polymorphonuclear leukocytes, resulting in excess generation of reactive oxygen and nitrogen species. If the detrimental effects of these reactive species are not limited by antioxidant systems, cellular damage and subsequent cell death and necrosis might develop by several mechanisms, especially including the peroxidation of cellular membrane lipids and oxidation of cellular proteins [6,7].

Phospholipids are essential constituents of cell membranes with pivotal roles in maintenance of homeostasis, cell compartmentalization, and enzymatic activities associated with membrane systems and coupling between receptor and intracellular signaling [8]. Phospholipids also contribute to the roles of intestinal mucus in constituting a continuous layer at the luminal side of the mucus gel. Phosphatidylcholine (PC) is the major lipid of the GI mucus layer; it establishes a protective hydrophobic surface. This intestinal surfactant also has a key role in mucosal defense. The defective PC layer was suggested to contribute to the development of inflammation [9]. Necrotizing enterocolitis was also reported to result in significant disruption of the intestinal barrier, which is associated with mucosal injury [10].

Cytidine 5'-diphosphocholine (CDP-choline) is an endogenous intermediate in the biosynthesis of the major membrane phospholipid PC via the Kennedy pathway [11]. Given exogenously, part of CDP-choline is metabolized in the circulation to cytidine and choline [12-15], which are then used for phospholipid synthesis [16] via the Kennedy pathway [9].

Although previous studies reported the beneficial effects of CDP-choline treatment in several neurodegenerative conditions as well as hypoxia-ischemia and stroke $[17,18]$, to 
the best of our knowledge, no study has evaluated the effects of CDP-choline on intestinal ischemia, inflammation, and epithelial injury, a condition occurring in NEC. Therefore, the aim of this study was to investigate the possible cytoprotective effect of CDP-choline administration on inflammation, intestinal cell death, and apoptosis in a neonatal rat model of NEC.

\section{Materials and methods}

\subsection{Animals and experimental design}

We carried out this study out according to the guidelines of the Institutional Committee on the Care and Handling of Animals of the National Laboratory; it was approved by the Experimental Animal Ethics Committee at Gulhane Military Medical Academy, Ankara, Turkey. We kept pregnant rats in identical cages and gave them free access to regular laboratory chow and water ad libitum. We equally divided 30 newborn pups that were delivered spontaneously from pregnant Sprague-Dawley rats into three groups as follows: control (left with the mothers, breastfed freely and not subjected to stress), NEC (subjected to NEC and received saline intraperitoneally for $3 \mathrm{~d}$ ), and NEC + CDP-choline (subjected to NEC and received CDP-choline intraperitoneally for $3 \mathrm{~d}$ ).

We prepared the NEC model by formula feeding, hypoxia, and cold stress, as described previously [6]. Briefly, rat pups in NEC and NEC + CDP-choline groups were immediately separated from their mothers to avoid the preventive effects of mothers' milk, and were kept at $37^{\circ} \mathrm{C}$ in a humidified incubator. We used a special rodent formula (15 g Similac 60/40; Ross Pediatrics, Columbus, $\mathrm{OH}$ ) prepared with $75 \mathrm{~mL}$ puppy- canine milk (Beaphar-Bogena; BV Sedel, The Netherlands) to induce NEC. Feeding was started at $0.2 \mathrm{~mL}$ every $3 \mathrm{~h}$ and increased gradually each time with $0.1-\mathrm{mL}$ increments daily as tolerated. We also exposed these pups to $100 \% \mathrm{CO}_{2}$ inhalation for $10 \mathrm{~min}, 4^{\circ} \mathrm{C}$ cold exposure for $5 \mathrm{~min}$, and $97 \% \mathrm{O}_{2}$ for 5 min twice daily for $3 \mathrm{~d}$, for NEC development. We weighed all pups daily and recorded changes in their weight.

Pups in the NEC + CDP-choline group received CDP-choline (Sigma, St. Louis, MO) dissolved in $0.9 \%$ saline by intraperitoneal route at a dose of $300 \mathrm{mg} / \mathrm{kg} / \mathrm{d}$; those in the NEC group received $0.9 \%$ saline at a volume of $2 \mathrm{~mL} / \mathrm{kg} / \mathrm{d}$ from the first day through the third day of the study during the NEC procedure. An observer blinded to the protocol assessed the rats in terms of their behavior, using a modified neonatal rat clinical sickness score ( 0 , best; 12 , worst), including appearance, response to touch, natural activity, and color for each feeding sessions on each day, as previously described [19]. Number of deaths, approximate death time, and cause of death (owing to NEC or iatrogenic during the procedure) were recorded daily for all groups.

In a separate experiment, we tested the long-term effects in terms of weight changes and clinical sickness score, as well as survival after NEC lesion. For this purpose, we 
randomly arranged 30 rat pups into the control, NEC, and NEC + CDP-choline groups and followed the experimental procedure described above. We evaluated weight changes and clinical sickness scores in these pups on days $3,5,7,10$, and 14 postnatally and used the same time points to record the number of surviving pups.

\subsection{Macroscopic gut assessment}

We killed all pups on the fourth day of the study under deep anesthesia using ketaminexylazine (100 and $10 \mathrm{mg} / \mathrm{kg}$, respectively). After we killed the pups, we opened the abdominal cavity and dissected the GI tract from duodenum to rectum free from the surrounding tissue; we straightened out the gut and placed it on a Petri dish containing phosphate-buffered saline ( $\mathrm{pH}$ 7.2). We performed the macroscopic assessment of the gut to confirm NEC using a new scoring system based on gut color, consistency, and dilatation [19].

\subsection{Immunohistochemistry}

After macroscopic evaluation, we excised $2 \mathrm{~cm}$ of terminal ileum and fixed it with $4 \%$ paraformaldehyde in $0.1 \mathrm{~mol} / \mathrm{L}$ phosphate buffer for histopathological examination and apoptosis scoring, which were evaluated in a blinded fashion. We then excised the rest of the small intestines, washed them with physiological saline, and allocated them for biochemical analyses.

We embedded fixed intestinal tissues in paraffin and sliced the paraffin blocks into 4to 5-mm sections. We stained slides with hematoxylin-eosin for histological evaluation and with transferase-mediated dUTP nick end labeling (TUNEL) using the in situ cell death detection POD kit (Roche Molecular Biochemicals, Mannheim, Germany) to evaluate apoptosis. We chose sections according to a systematic random sampling procedure and mounted them onto poly-L-lysine-coated slides (Histobond adhesion slides; Paul Marienfeld $\mathrm{GmbH} \&$ Co., Lauda-Konigshofen, Germany). We heated these sections at $60^{\circ} \mathrm{C}$ for $45 \mathrm{~min}$ followed by washing in xylene and rehydration through a graded series of alcohol (ethanol 100\%, 95\%, 80\%, and 70\%) and then washed them in distilled water.

We then incubated the sections in $20 \mathrm{mg} / \mathrm{mL}$ proteinase $\mathrm{K}$ solution (Roche Applied Sciences, Penzberg, Germany) for $20 \mathrm{~min}$ at room temperature and immersed them in $3 \% \mathrm{H}_{2} \mathrm{O}_{2}$ in methanol for 10 min. After this procedure, we performed permeabilization with Triton X (Sigma-Aldrich, St. Louis, MO) for 10 min on ice. We then incubated the sections with the TUNEL reaction mixture at $37^{\circ} \mathrm{C}$ in a humidified chamber for $1 \mathrm{~h}$ followed by converter-POD treatment at $37^{\circ} \mathrm{C}$ for $30 \mathrm{~min}$. We analyzed immunofluorescence reaction and photographed it with an Olympus BX50 photomicroscope before the POD treatment. We used diaminobenzidine tetrahydrochloride (diaminobenzidine/metal concentrate: peroxidase buffer; Roche Applied Sciences) as the chromogene, counterstained the slides with hematoxylin (Harris' hematoxylin; Sigma-Aldrich), and coverslipped them (DPX mountant; Fluka, St. Louis, MO) 
for light microscopy. We also incubated alternate sections in the absence of a TUNEL reaction mixture for negative controls. Histological findings were graded as follows: grade 0 , normal; grade 1 , focal mild injury confined to villous tips; grade 2, partial or total loss of villi; grade 3, necrosis extending to the submucosa; and grade 4, transmural necrosis [20]. We evaluated apoptosis as follows: grade 0, normal; grade 1, apoptotic nuclei presenting at villous tips; grade 2, apoptotic nuclei covering all villous tips but crypts protected; and grade 3, transmural spread of apoptotic nuclei [21].

\subsection{Active caspase-3 expression}

We homogenized the remaining intestinal tissues that were not subjected to fixation in 50 vol ice-cold phosphate-buffered saline ( $\mathrm{pH}$ 7.2) using a homogenizer (T18 basic UltraTurrax; IKA, Staufen, Germany). We used aliquots of homogenates for total protein analysis by bicinchoninic acid assay (Thermo Fisher Scientific, Rockford, IL). Aliquots of homogenates were mixed with equal volumes of Laemmli loading buffer and boiled for 5 min before gel electrophoresis. We loaded equal amounts of protein and separated them using sodium dodecyl sulfate-polyacrylamide gel electrophoresis (4e20\%; Bio-Rad, Hercules, CA). We then transferred proteins onto polyvinylidene fluoride membranes (Immobilon-P; Millipore, Billerica, MA). The remaining binding sites were blocked with $4 \%$ nonfat dry milk (Carnation, Glendale, CA) for 30 min in Tris-buffered saline and Tween 20 (TBST). We then rinsed membranes five times in TBST buffer and incubated them overnight in TBST solution containing the primary antibody against active caspase- 3 (Abcam, Cambridge, MA) and b-actin (Abcam), which we used as a loading control for intestinal homogenates. After overnight incubation and five rinses in TBST buffer, we incubated blots for $1 \mathrm{~h}$ with the appropriate peroxidase-linked secondary antibody (GE Healthcare, Waukesha, WI). We then rinsed blots in TBST buffer five times, and detected and visualized protein-antibody complexes using the enhanced chemiluminescence system (GE Healthcare) and developed them on Kodak X-AR film (Rochester, NY). We digitized films using a Supervista S-12 scanner with a transparency adapter (UMAX Technologies, Freemont, CA). We compared immunoreactive bands densitometrically using the Public Domain NIH Image program available on the internet at http://rsbweb.nih.gov/ij/download.html. Areas under the absorbance curve are expressed as arbitrary units and normalized as percentages of those generated in the same blot using samples from intestines of control animals.

\subsection{Measurement of intestinal tissue cytokine content}

We centrifuged aliquots of homogenates at $12,000 \mathrm{~g}$ for $20 \mathrm{~min}$ at $4^{\circ} \mathrm{C}$ and used cell-free supernatants to determine intestinal tissue proinflammatory cytokine (TNF- $\alpha$, interleukin [IL]-6, and IL-1 $\beta$ ) contents by specific enzyme-linked immunosorbent assay kits (R\&D Systems, Minneapolis, MN), as described previously [22]. 


\subsection{Biochemical analyses}

We measured the activity of superoxide dismutase (SOD), glutathione peroxidase (GSH$\mathrm{Px}$ ), and myeloperoxidase (MPO), as well as the malondialdehyde (MDA) content of intestinal tissues, using cell-free supernatants of tissue homogenates by spectrophotometric analyses (UV-1700; Shimadzu, Kyoto, Japan).

We determined tissue SOD activity using a method involving spectrophotometric detection of formazan production at $550 \mathrm{~nm}$ by inhibiting nitroblue tetrazolium reduction with xanthine-xanthine oxidase used as a superoxide generator, as described previously [23]. We also determined tissue GSH-Px activity by dithiodinitrobenzoic acid method at an absorbance of $412 \mathrm{~nm}$, as described previously [24]. Tissue MDA content was determined by a method based on the reaction of MDA with thiobarbituric acid at $95^{\circ} \mathrm{C}$, as described previously [25]. We determined tissue MPO activity using a method based on the reduction of 5-thio-2-nitrobenzoic acid to 5-5-dithiobis (2-nitrobenzoic acid) by following the decrease in absorbance at $412 \mathrm{~nm}$, as described previously [26]. We extracted intestinal phospholipids and measured the according to the method of Folch et al [27]. Briefly, we mixed $1 \mathrm{~mL}$ intestinal homogenate with $3 \mathrm{~mL}$ of a chloroform/methanol mixture $(2: 1 \mathrm{v} / \mathrm{v})$ and vortexed it, followed by sequential addition of $3 \mathrm{~mL}$ chloroform/methanol mixture $(2: 1 \mathrm{v} / \mathrm{v})$ and $1 \mathrm{~mL}$ ice-cold deionized water. The mixture was allowed to stand overnight at $4^{\circ} \mathrm{C}$. Next day, we separated the organic (lower) and aqueous (upper) phases of the mixture by centrifugation ( $10 \mathrm{~min}$ at $4^{\circ} \mathrm{C} ; 1000 \mathrm{~g}$ ). Aliquots (0.1-0.4 mL) of the organic phase were dried under vacuum. Residues of $0.4-\mathrm{mL}$ aliquots of the organic phase were reconstituted in methanol and subjected to thin-layer chromatography using silica G plates (Adsorbosil Plus-1; Alltech, Nicholasville, KY) and a system consisting of chloroform/ethanol/triethylamine/water (30:34:30:8) as the mobile phase. We used phospholipid standards to identify the corresponding bands under ultraviolet light after we sprayed the plates with $0.1 \%$ diphenylhexatriene in petroleum ether. Bands for individual phospholipid classes such as PC, phosphatidylethanolamine $(P E)$, phosphatidylserine (PS), sphingomyelin (SM), and phosphatidylinositol (PI) were scraped off the plates and extracted into $1 \mathrm{~mL}$ methanol, dried under vacuum, and assayed for phosphorus content. We assayed aliquots of intestinal homogenates for protein using a bicinchoninic acid reagent (Perkin Elmer, Norwalk, CT) and present phospholipid levels as nanomoles per milligram of protein.

\subsection{Statistical analysis}

We performed statistical analyses using SPSS statistical software (version 16.0; Chicago, IL). Data are expressed as the means \pm standard error of mean. We analyzed biochemical and pathological parameters by analysis of variance, followed by appropriate post hoc tests including multiple-comparison and Holm-Sidak test. We used the Kruskal-Wallis one-way analysis of variance by ranks followed by Bonferroni correction for a simultaneous statistical test of the pathologic score for the NEC and NEC + CDP-choline 
groups. We compared the groups using the Mann-Whitney nonparametric test for independent samples. $\mathrm{P}<0.05$ was considered statistically significant.

\section{Results}

Three rat pups (two in the NEC group and one in the NEC + CDP-choline group) died during the experimental procedures. The mean birth weight of pups did not differ significantly between study groups and the control group. However, the mean weight of the pups in the control group $(8.1 \pm 0.4 \mathrm{~g})$ and NEC + CDP-choline group $(6.3 \pm 0.2 \mathrm{~g})$ were significantly greater than that of the pups in the NEC group $(5.4 \pm 0.3$ g) (both $P<0.05$ ) on the fourth day, when the study was terminated.

The mean clinical sickness score of the pups at the end of the study was significantly lower in the NEC + CDP-choline group compared with the NEC group ( $8.6 \pm 3.4$ versus 4.6 $\pm 1.2)(P=0.001)$, indicating a better clinical status in pups that received CDP-choline. Similarly, the mean macroscopic gut assessment score was significantly lower in the NEC + CDP-choline group compared with the NEC group ( $5 \pm 2$ versus $2 \pm 1$ ), indicating less intestinal tissue damage in the treatment group $(P=0.01)$. We found no evidence of macroscopic changes in tissues of rats in the control group (Table 1). We detected intestinal necrosis and/or perforation in the post-mortem evaluation of intestinal tissues of rats that died during the experimental procedures.

Intestinal injury scoring performed according to the histopathological evaluation also showed that the grade of histopathological damage was significantly greater in the NEC group compared with the NEC + CDP-choline group (mean score, $2.6 \pm 0.4$ versus $1.5 \pm$ $0.2)(P=0.003)$. We found no histological damage in tissue samples of the control group (Table 1).

The mean apoptosis scores were significantly lower in the NEC + CDP-choline group compared with those in the NEC group (mean score, $2.9 \pm 0.6$ versus $1.6 \pm 0.4)(P=0.002)$ (Table 1). Figure 1 shows immunofluorescence images of TUNEL stains, as well as light microscopic images of TUNEL-diaminobenzidine tetrahydrochloride stains for the control, NEC, and NEC + CDP-choline groups. In addition, although the expression of active caspase-3, a biomarker of apoptosis, was doubled in the NEC group compared with the control group, it was reduced by about 30\% in the NEC + CDP-choline group (Fig. 2) $(P<0.05)$. Levels of $\beta$-actin, a protein used as a protein loading control for gel electrophoresis, were not statistically different between groups.

Whereas the levels of IL-1 $\beta, I L-6$, and TNF- $\alpha$ were significantly increased in the NEC group compared with those in the control group $(P<0.05)$, CDP-choline treatment significantly reduced the inflammation-associated elevations of IL- $1 \beta$ (from $1354 \pm 88$ to $1142 \pm 65 \mathrm{pg} / \mathrm{mg}$ protein), IL-6 (from $541 \pm 28$ to $436 \pm 25 \mathrm{pg} / \mathrm{mg}$ protein), and TNF- $\alpha$ (from $567 \pm 30$ to $474 \pm 26 \mathrm{pg} / \mathrm{mg}$ protein) $(\mathrm{P}<0.05)$ (Table 2$)$, which suggests an antiinflammatory effect of CDP-choline treatment in the neonatal rat model of NEC. 
Tissue GSH-Px and SOD activities were significantly preserved in the NEC + CDPcholine group compared with those in the NEC group, which indicates the effect of CDPcholine on decreasing oxidative stress $(P<0.01$ ) (Table 3$)$. In addition, tissue MDA levels, as well as MPO and XO activities in the NEC + CDP-choline group, were significantly lower than those in the NEC group, which suggests decreased lipid peroxidation after CDPcholine treatment. However, MDA levels, as well as MPO and XO activities in intestinal tissues in the control group, were still significantly greater than those in the NEC + CDPcholine group $(P<0.01$ ) (Table 3$)$.

Levels of total phospholipids, as well as those of the individual phospholipid classes PC, PE, PS, SM, and PI were reduced significantly in intestinal tissues of pups in the NEC group compared with the control group $(\mathrm{P}<0.01$ for total phospholipids, $\mathrm{PC}$, and $\mathrm{PE} ; \mathrm{P}<$ 0.05 for PS, SM, and PI) (Table 4). In addition, CDP-choline treatment after NEC lesion significantly increased levels of total phospholipids and PC $(P<0.05$ for both). However, increases in levels of other phospholipid classes after CDP-choline treatment did not reach statistical significance (Table 4).

Table 1- Comparison of the study and control groups in terms of weight change, clinical sickness score, macroscopic assessment score, histopathological evaluation, and mortality.

\begin{tabular}{lccc}
\hline Variable & $\begin{array}{c}\text { Control group } \\
(\mathrm{n}=10)\end{array}$ & $\begin{array}{c}\text { NEC group } \\
(\mathrm{n}=10)\end{array}$ & $\begin{array}{c}\text { NEC + CDP-choline } \\
\text { group }(\mathrm{n}=10)\end{array}$ \\
\hline Birth weight (g), mean \pm SD & $5.2 \pm 0.4$ & $5.3 \pm 0.5$ & $5.2 \pm 0.5$ \\
Weight at the end of the study, mean \pm SD & $8.1 \pm 0.4$ & $5.4 \pm 0.3^{*}$ & $6.3 \pm 0.2^{\mathrm{y}}$ \\
Clinical sickness score at the end of the study, & 0 & $8.6 \pm 3.4^{z}$ & $4.6 \pm 1.2^{\mathrm{y}}$ \\
mean \pm SD & & $5 \pm 2^{z}$ & $2 \pm 1^{\mathrm{y}}$ \\
Macroscopic assessment score, mean \pm SD & 0 & $2.6 \pm 0.4^{*}$ & $1.5 \pm 0.2^{\mathrm{y}}$ \\
Intestinal injury score, mean \pm SD & 0 & $2.9 \pm 0.6^{*}$ & $1.6 \pm 0.4^{\mathrm{y}}$ \\
Apoptosis score, mean \pm SD & $0(0-1)$ & $2(20)$ & $1(10)$ \\
Mortality, $n$ (\%) & 0 & &
\end{tabular}

${ }^{*} P<0.05$ compared with the control group.

y $P<0.05$ compared with the hyperoxia group using one-way analysis of variance followed by post-hoc HolmSidak test.

${ }^{z} P<0.01$ compared with the control group. 

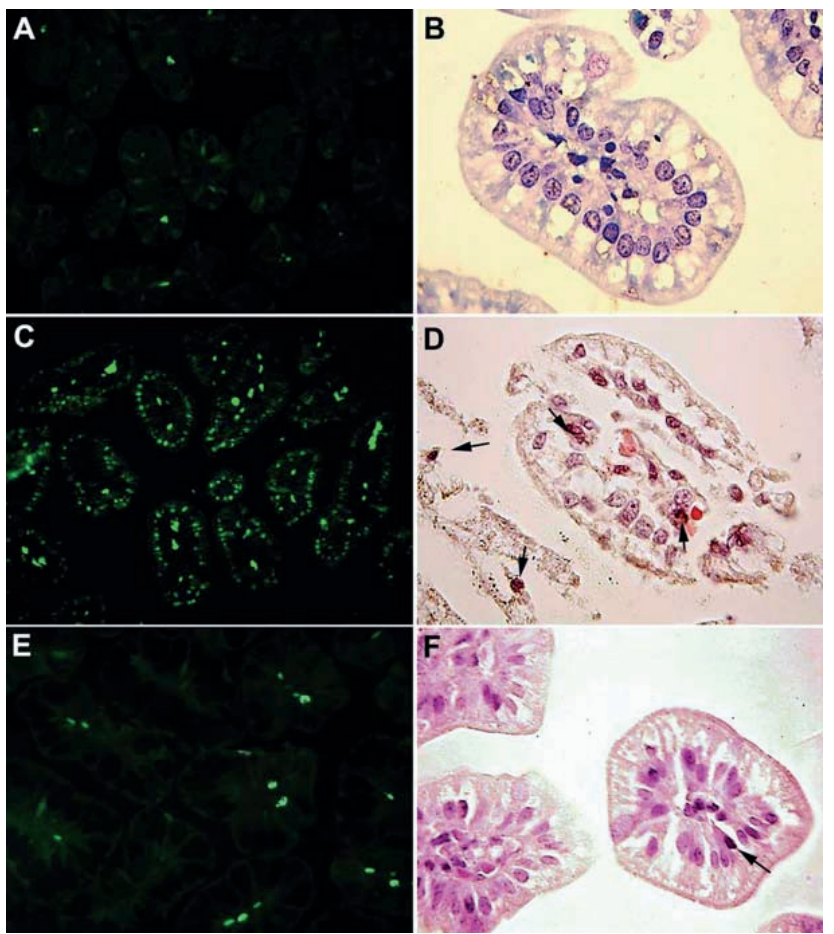

Fig. 1- Representative images of immunofluorescence stains for the control (A), NEC (C), and NEC + CDP-choline (E) groups (x40 magnification) and light microscopic images of TUNEL-diaminobenzidine tetrahydrochloride stains for the control (B), NEC (D), and NEC + CDP-choline (F) groups (x100 magnification). Arrows in (D) and (F) indicate apoptotic cells. (Color version of figure is available online.)

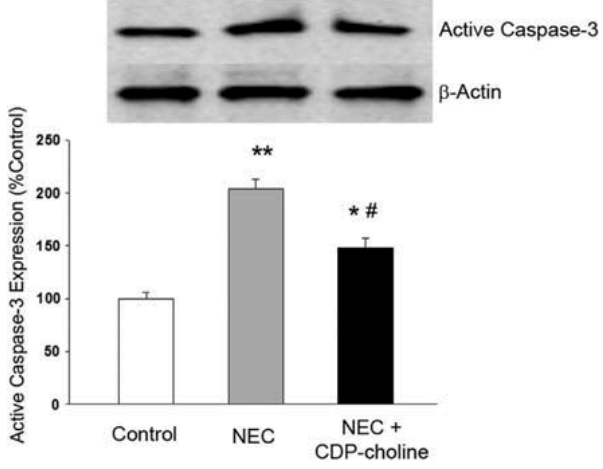

Fig. 2- Changes in active caspase-3 expression in relation to the control group in the NEC and NEC + CDP-choline groups. ${ }^{*} \mathrm{P}<0.05$ and ${ }^{* *} \mathrm{P}<0.01$ compared with the control group; $\# \mathrm{P}<0.05$ compared with the NEC group using one-way analysis of variance followed by post-hoc Holm-Sidak test. 
Table 2- Levels of cytokines in intestinal tissue homogenates.

\begin{tabular}{|c|c|c|c|}
\hline $\begin{array}{l}\text { Cytokine levels } \\
\text { (pg/mg protein) }\end{array}$ & Control group & NEC group & $\begin{array}{c}\mathrm{NEC}+ \\
\text { CDP-choline group }\end{array}$ \\
\hline IL-1 $\beta$ & $985 \pm 47$ & $1354 \pm 88^{*}$ & $1142 \pm 65^{y}$ \\
\hline IL-6 & $390 \pm 21$ & $541 \pm 28^{*}$ & $436 \pm 25^{y}$ \\
\hline TNF- $\alpha$ & $452 \pm 24$ & $567 \pm 30^{2}$ & $474 \pm 26^{v}$ \\
\hline
\end{tabular}

Table 3-Intestinal tissue MDA content and SOD, GSH-Px, MPO, and XO activities.

\begin{tabular}{lccc}
\hline $\begin{array}{l}\text { Biochemical } \\
\text { analyses }\end{array}$ & $\begin{array}{c}\text { Control group } \\
(\mathrm{n}=10)\end{array}$ & $\begin{array}{c}\text { NEC group } \\
(\mathrm{n}=10)\end{array}$ & $\begin{array}{c}\text { NEC + CDP-choline } \\
\text { group }(\mathrm{n}=10)\end{array}$ \\
\hline MDA (nmol/g protein) & $1.3 \pm 0.2$ & $1.9 \pm 0.2^{*}$ & $1.4 \pm 0.3^{\mathrm{y}}$ \\
SOD (U/mg protein) & $109.6 \pm 7.4$ & $71.0 \pm 2.8^{*}$ & $108.0 \pm 13.3^{\mathrm{y}}$ \\
GSH-Px (U/mg protein) & $12.5 \pm 1.6$ & $5.5 \pm 1.0^{*}$ & $11.0 \pm 1.2^{\mathrm{y}, z}$ \\
MPO (U/g protein) & $61.6 \pm 6.1$ & $148 \pm 6.8^{*}$ & $93 \pm 2.5^{*}, z^{*}$ \\
XO (U/mg protein). & $0.8 \pm 0.1$ & $3.2 \pm 0.2^{*}$ & $1.3 \pm 0.2^{\mathrm{y}, z}$ \\
\hline
\end{tabular}

${ }^{*} P<0.01$ compared with the control group.

v $P<0.05$ compared with the control group.

z $P<0.05$ compared with the NEC group using one-way analysis of variance followed by post-hoc Holm-Sidak test

Table 4- Levels of intestinal phospholipids.

\begin{tabular}{|c|c|c|c|c|c|c|}
\hline \multirow[t]{2}{*}{ Group } & \multicolumn{6}{|c|}{ Intestinal phospholipids (nmol/mg protein) } \\
\hline & Total PL & PC & $\mathrm{PE}$ & PS & SM & $\mathrm{Pl}$ \\
\hline Control & $198 \pm 6$ & $86 \pm 5$ & $51 \pm 2$ & $20 \pm 3$ & $11.6 \pm 0.7$ & $4.2 \pm 0.2$ \\
\hline NEC & $121 \pm 5^{*}$ & $48 \pm 3^{*}$ & $20 \pm 3^{*}$ & $13 \pm 2^{y}$ & $6.9 \pm 0.9^{y}$ & $3.6 \pm 0.1^{y}$ \\
\hline NEC + CDP-choline & $166 \pm 10^{z}$ & $70 \pm 3^{2}$ & $29 \pm 2^{y}$ & $14 \pm 2^{y}$ & $8.6 \pm 0.7^{y}$ & $3.8 \pm 0.1^{y}$ \\
\hline
\end{tabular}

$\mathrm{PL}=$ phospholipid; $\mathrm{PC}=$ phosphatidylcholine; $\mathrm{PE}=$ phosphatidylethanolamine; $\mathrm{PS}=$ phosphatidylserine; $\mathrm{SM}=$ sphingomyelin; $\mathrm{PI}=$ phosphatidylinositol.

${ }^{*} P<0.01$ compared with the control group.

v $P<0.05$ compared with the control group.

z $P<0.05$ compared with the NEC group using one-way analysis of variance followed by post-hoc Holm-Sidak test. 
In separate experiments, in which we investigated long- term consequences of NEC lesion, we found that compared with the control group, birth weights were significantly reduced and clinical sickness scores were significantly enhanced from days 3 through 7 in the NEC group, whereas CDP-choline treatment ameliorated the reduction in birth weight and the enhancement in clinical sickness scores (Table 5). We also found that no pups in the NEC group survived after day 7 in the further follow-up, whereas seven of 10 pups in the CDP-choline group survived through day 14, when the survival experiment was completed (Fig. 3).

Table 5- Comparison of weights and clinical sickness scores during 14-d follow-up.

\begin{tabular}{lccc}
\hline Variable & Control & NEC & $\begin{array}{c}\text { NEC }+ \\
\text { CDP-choline }\end{array}$ \\
\hline Birth weight (g) Weight & $5.21 \pm 0.42$ & $5.23 \pm 0.35$ & $4.97 \pm 0.20$ \\
Day 3 (g) & $6.78 \pm 0.30$ & $5.06 \pm 0.13^{*}$ & $5.68 \pm 0.14^{*}, y$ \\
Day 5 (g) & $8.68 \pm 0.81$ & $4.85 \pm 0.11^{*}$ & $7.20 \pm 0.33^{*}, y$ \\
Day 7 (g) & $11.78 \pm 1.18$ & $4.80 \pm 0.10^{*}$ & $8.44 \pm 0.44^{*}, y$ \\
Day 10 (g) & $16.81 \pm 0.65$ & & $13.84 \pm 0.86^{*}$ \\
Day 14 (g) & $21.26 \pm 1.04$ & & $19.00 \pm 1.36^{*}$ \\
Clinical sickness score & & & \\
Day 3 & $0.00 \pm 0.00$ & $5.43 \pm 0.79^{*}$ & $3.89 \pm 0.78^{*}, y$ \\
Day 5 & $0.00 \pm 0.00$ & $6.33 \pm 1.16^{*}$ & $3.44 \pm 0.53^{*}, y$ \\
Day 7 & $0.00 \pm 0.00$ & $7.67 \pm 0.58^{*}$ & $2.78 \pm 0.67^{*}, y$ \\
Day 10 & $0.00 \pm 0.00$ & & $1.78 \pm 0.67^{*}$ \\
Day 14 & $0.00 \pm 0.00$ & & $0.00 \pm 0.00$ \\
\hline
\end{tabular}

${ }^{*} \mathrm{P}<0.01$ compared with the control group.

y $P<0.01$ compared with the NEC group using one-way analysis of variance followed by post-hoc Holm-Sidak test.

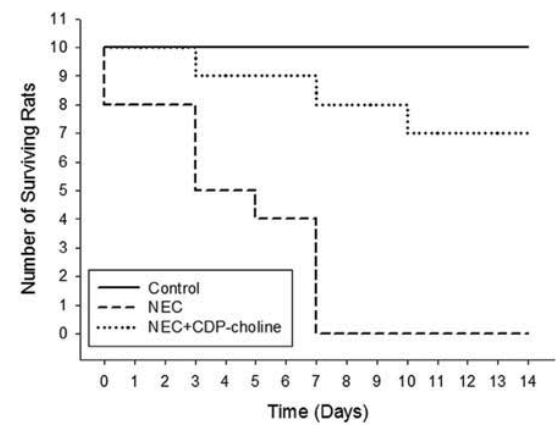

Fig. 3- Number of surviving rat pups in the control, NEC, and NEC + CDP-choline groups

\section{Discussion}

These data show that CDP-choline administration reduces the clinical sickness score, ameliorates macroscopic and histopathological intestinal injury, decreases inflammation 
and apoptosis, and increases intestinal tissue phospholipid levels in a neonatal rat model of NEC. Cytidine 5'-diphosphocholine therapy also decreases lipid peroxidation and oxidant status, while increasing antioxidant status in this model. To the best of our knowledge, this is the first experimental study that showed beneficial effects, both in the short and long term, of CDP-choline treatment on NEC development in newborn rats. Necrotizing enterocolitis is the most common Gl emergency, with a considerable rate of morbidity and mortality in neonates, particularly, in preterm infants. Despite recent improvements in neonatal intensive care, no significant advances have been achieved in the prevention, incidence, and mortality of NEC [2]. Therefore, newer therapeutic approaches are required to prevent and treat NEC [28].

Necrotizing enterocolitis is a multifactorial disease with a poorly understood complex pathophysiology. A combination of genetic predisposition, intestinal immaturity, abnormal intestinal microbial colonization, and highly immunoreactive intestinal mucosa was suggested to lead to a confluence of predisposing factors [29]. In a recent National Institute of Child Health and Human Development workshop on NEC research, NEC was suggested to arise from an uncontrolled exuberant inflammatory response to bacterial colonization in the intestine of the preterm infants [30]. It was also suggested to represent overactivation of the immune system to insults such as ischemia, infection, enteral feeding, and abnormal bacterial colonization, leading to intestinal epithelial disruption, bacterial translocation, and overreaction of immature intestinal epithelial cells. These events might activate stress pathways and lead to suppression of inhibitory pathways, finally resulting in unfettered inflammatory response with harmful effects [31]. Therefore, if the detrimental effects of these reactive species cannot be limited by antioxidant systems, cellular damage and subsequent cell death and necrosis might develop by several mechanisms, including, in particular, peroxidation of cellular membrane lipids and oxidation of cellular proteins $[6,7]$.

Phospholipids are essential constituents of cell membranes and are involved in membrane functions such as maintenance of cellular homeostasis, receptor functioning, and enzymatic activities [8]. Ischemic or hypoxic insults to tissues disrupt membrane integrity by several mechanisms including phospholipid breakdown by several phospholipases [8]. Therefore, phospholipid synthesis is crucially important under such circumstances. There is also increasing evidence that the mucus layer in the GI tract is a key factor for protection of the epithelia from luminal aggressors, microorganisms, and their noxious products [32]. Although phospholipids account for a minor part of GI mucus, they are indispensable in the maintenance of an intact barrier function [33]. Phosphatidylcholine, which represents the major phospholipid class with a ratio of $35 \%$ $72 \%$ [33], is largely responsible for the establishment of the hydrophobic surface of the GI mucosa. In addition, PC was reported to interfere with cell signaling by attenuating apoptosis, and also showed anti-inflammatory properties [34,35]. Exogenous PC inhibited mucosal damage caused by acids and other noxious agents in the GI system [36,37]. Therefore, it was suggested that luminal PC had two possible functions: to establish the 
surface hydrophobicity and to modulate the inflammatory signaling system [38]. Also, altered concentration and composition of PC were reported in patients with inflammatory bowel disease, and exogenous PC administration was shown to have a significant therapeutic effect in the treatment of ulcerative colitis $[33,39,40]$. In the present study, we found that CDP-choline treatment enhanced levels of total phospholipids and PC in intestinal tissues of rat pups subjected to NEC lesion. These data are in good accord with the abovementioned previous studies that suggest a therapeutic effect of PC in intestinal injury models. Therefore, one mechanism by which CDP-choline exerts protective effects in a neonatal rat model of NEC might be the enhancement of PC levels in intestinal tissue.

Necrotizing enterocolitis usually occurs in the small intestinal tract of preterm infants, and intestinal mucus protects the epithelial layer. As stated above, mucins are large glycoproteins that provide a physical barrier, facilitate removal of adherent bacteria, and concentrate enzymes near the epithelial surface to aid in host nutrient digestion [41]. The ileal portion of the small intestine expresses multiple mucin genes including Muc1, Muc3, Muc4, and Muc17, whereas Muc2 is the most abundant mucin in the small intestine that is secreted into the lumen [42]. Decreased Muc2-stained goblet cells was reported described in a rat model of NEC [10]; and in a recent study, mucus-containing goblet cells and Paneth cells also were significantly decreased in infants with NEC [43]. It is therefore possible that the beneficial effects of CDP-choline in our study could have resulted from enhanced PC and total phospholipid synthesis, and hence cellular membrane synthesis, which could have served to protect membrane integrity in rat pups with intestinal injury.

It is widely accepted that elevated cytokines, major contributors to the inflammatory response, are principal modulators of the release of reactive oxygen species during NEC [44]. Therefore, treatments targeting the inflammatory response might be beneficial in NEC. Recent studies have demonstrated anti-inflammatory properties of efferent vagus nerve activation, and the term "cholinergic antiinflammatory pathway" has been proposed [45]. It was also suggested that acetylcholine released from nonneuronal cells in the mucosa might target the muscarinic receptors there, and that mechanisms related to the noncholinergic system have a role in the remodeling process that occurs in the mucosa in inflammatory bowel diseases [46]. Cytidine 5'-diphosphocholine is a choline donor, because part of exogenous CDP-choline is metabolized to choline $[12,13]$. Choline is also a constituent of membranes and a precursor of the neurotransmitter acetylcholine [15]. Whereas choline deficiency results from increased levels of proinflammatory cytokines such as TNF- $\alpha$ [47], parenteral choline administration attenuates endotoxininduced multiple organ injury and the elevation in circulating TNF- $\alpha$ levels [48]. Phosphatidylcholine was also shown to inhibit TNF- $\alpha$-induced proinflammatory responses by changing the compartmentation of TNF receptors to lipid rafts, inhibiting nuclear factor-kB activation, and preventing mitogen-activated protein kinase phosphorylation and subsequent activation [49]. In addition, mucus samples of patients with ulcerative colitis, a chronic inflammatory disorder of the colon, contained reduced 
levels of PC, and the anti-inflammatory effects of PC were suggested to block TNF- $\alpha$ induced proinflammatory signaling $[35,50]$. In accordance with these findings, CDPcholine significantly reduced TNF- $\alpha$, IL1- $\beta$, and IL- 6 levels in the intestinal tissue of rat pups subjected to NEC, an effect that might well be mediated by elevated PC levels found in our study.

Pathophysiologic changes in intestinal ischemia may also involve activation of the immune system by the release of inflammatory cytokines, resulting in excess generation of reactive oxygen and nitrogen species [44]. Under such circumstances including NEC, activities of antioxidant enzymes GSH-px and SOD are reduced, whereas activities of free radical-generating enzymes $\mathrm{XO}$ and MPO as well as the MDA content are increased, which indicates enhanced oxidant stress [51,52]. In our study, CDP-choline treatment enhanced decreased GSH-Px and SOD activities, whereas it reduced tissue MDA content as well as MPO and XO activities in a neonatal rat NEC model. This suggests that CDP-choline reduced the severity of NEC by reducing lipid peroxidation. In good agreement with our findings, oral PC pretreatment decreased ischemia-reperfusion-induced methane generation and intramucosal $\mathrm{pH}$, and diminished MPO activity and the anti-inflammatory influence in the small intestine [53].

These observations suggest that CDP-choline might prevent membrane destruction, decrease free radical generation, and preserve natural mechanisms against oxidative stress [17]. Because CDP-choline is a source of choline and its administration causes bodily responses of a cholinergic nature [54,55], it could be speculated that one mechanism by which CDP-choline reduces free radical generation and the severity of NEC might be the action of choline that is released by CDP-choline breakdown on cytokine production. However, this hypothesis needs further investigation.

Besides membrane breakdown and generation of reactive species, exposure to stress may also result in increased apoptosis in gastric and intestinal epithelial cells in neonatal rats $[56,57]$. Intestinal epithelium has a high turnover rate that necessitates the removal of apoptotic cells and a greater number of TUNEL-positive nuclei within the intestinal epithelium of animals exposed to hypoxia-reoxygenation [58]. Data obtained in our study showed that TUNEL-positive cell counts increased in neonatal rats in the NEC group, a finding that confirms previous observations. Cytidine 5' -diphosphocholine may confer benefit with regard to reducing apoptotic activity in cells subjected to ischemic and/or hypoxic insults. For example, CDP-choline decreased apoptosis in the brain by preventing caspase-3 activation by down-regulating the calpain-specific procaspase-3 breakdown product in a rat model of neonatal hypoxic-ischemic encephalopathy [59]. In good agreement with previous observations in brain tissue, we observed in our NEC model that CDP-choline treatment significantly reduced the TUNEL-positive cell count as well as active caspase-3 levels, which indicates a protective effect of CDP-choline on apoptosis in the injured intestine.

Vascular pathologies may also be associated with NEC. The diving seal reflex has been traditionally invoked as the mechanism responsible for intestinal ischemic injury and 
necrosis. In addition, significant reduction in the blood flows of superior mesenteric artery and abdominal aorta in asphyxiated newborn pigs, subsequently leading to mesenteric endothelial dysfunction, was implicated in increased vascular permeability, edema formation, and NEC development [60]. Hypoxia also causes intensive selective vasoconstriction, and blood is preferentially shunted from the gut and kidney to vital organs such as heart and brain in the neonate. This reflex vasospasm also occurs in mesenteric vessels, resulting in ischemia of the bowel. Beneficial effects of CDP-choline observed in our study might have resulted from CDP-choline's action on intestinal blood flow and/or systemic blood pressure. Previous studies showed that CDP-choline administration restores blood flow of superior mesenteric and renal arteries in a doseand time-dependent manner [61]. Moreover, intraperitoneal injection of CDP-choline increases systemic blood pressure in normal rats [10] and in rats subjected to hemorrhagic hypotension [62]. Therefore, CDP-choline might also provide prevention against NEC development by enhancing systemic blood pressure and restoring superior mesenteric artery flow.

In addition to short-term effects of CDP-choline administration in the present neonatal NEC model, we demonstrated that CDP-choline treatment enhances the mean weight of pups with NEC lesion and reduces their clinical sickness scores during long-term follow-up. We also showed that 7 of 10 pups that received CDP-choline survived for $14 \mathrm{~d}$ after NEC development, a considerable rate of survival. These data suggest that CDPcholine might be beneficial in terms of survival in the long term after NEC development. This long- term beneficial effect of CDP-choline may be associated with protection of intestinal epithelium and prevention of membrane destruction and apoptosis. The antiinflammatory and antioxidant effects of CDP-choline may also be involved in long-term survival.

In conclusion, this is the first study to show the beneficial effects of intraperitoneal CDP-choline treatment in a neonatal rat model of NEC. Cytidine 5'-diphosphocholine significantly reduced the clinical sickness score, ameliorated macroscopic and histopathological intestinal injury, decreased inflammation and apoptosis, and increased intestinal tissue PC and total phospholipid content. Cytidine 5'-diphosphocholine therapy also decreased lipid peroxidation and oxidant status, whereas it improved antioxidant status. The biochemical, morphological, and molecular biological effects of CDP-choline treatment seem to be associated with long-term consequences such as decreased clinical sickness scores and greater survival. Therefore, CDP-choline may be considered a new and potentially effective therapy in the prevention and treatment of NEC. 


\section{References}

1. Kafetzis DA, Shevaki C, Costalos C. Neonatal necrotizing enterocolitis: an overview. Curr Opin Infect Dis 2003;16:349.

2. Yurdakok M. What next in necrotizing enterocolitis? Turk J Pediatr 2008;50:1.

3. Martin CR, Walker WA. Probiotics: role in pathophysiology and prevention in necrotizing enterocolitis. Semin Perinatol 2008;32:127.

4. Martin CR, Walker WA. Intestinal immune defenses and the inflammatory response in necrotizing enterocolitis. Semin Fetal Neonatal Med 2006;11:369.

5. Frost BL, Jilling T, Caplan MS. The importance of pro- inflammatory signaling in neonatal NEC. Semin Perinatol 2008;32:100.

6. Guven A, Gundogdu G, Uysal B, et al. Hyperbaric oxygen therapy reduces the severity of necrotizing enterocolitis in a neonatal rat model. J Pediatr Surg 2009;44:534.

7. Li C, Jackson RM. Reactive species mechanisms of cellular hypoxia-reoxygenation injury. Am J Physiol Cell Physiol 2002; 282:C227.

8. Secades JJ. Citicholine: pharmacological and clinical review, 2010 update. Rev Neurol 2011;52:S1.

9. Stremmel W, Hanemann A, Ehehalt R, et al.Phosphatidylcholine (lecithin) and the mucus layer: evidence of therapeutic efficacy in ulcerative colitis. Dig Dis 2010;28:490.

10. Clark JA, Doelle SM, Halpern MD, et al. Intestinal barrier failure during experimental necrotizing enterocolitis: protective effect of EGF treatment. Am J Physiol Gastrointest Liver Physiol 2006;291:938.

11. Kennedy EP, Weiss SB. The function of cytidine coenzymes in the biosynthesis of phospholipides. J Biol Chem 1956;222:193.

12. Cansev M, Yilmaz MS, Ilcol YO, et al. Cardiovascular effects of CDP-choline and its metabolites: involvement of peripheral autonomic nervous system. Eur J Pharmacol 2007;577:129.

13. Wurtman RJ, Regan M, Ulus I, et al. Effect of oral CDP-choline on plasma choline and uridine levels in humans. Biochem Pharmacol 2000;60:989.

14. Cansev M. Uridine and cytidine in the brain: their transport and utilization. Brain Res Rev 2006;52:389.

15. Wurtman RJ, Cansev M, Ulus IH. Choline and its products acetylcholine and phosphatidylcholine. In: Lajtha A, editor. Neural lipids, handbook of neurochemistry and molecular neurobiology. Berlin: Springer-Verlag; 2009. p. 443.

16. Lopez-Coviella I, Agut J, Savci V, et al. Evidence that 5' cytidinediphosphocholine can affect brain phospholipid composition by increasing choline and cytidine plasma levels. J Neurochem 1995;65:889.

17. Adibhatla RM, Hatcher JF. Cytidine 5'-diphosphocholine (CDP-choline) in stroke and other CNS disorders. Neurochem Res 2005;30:15.

18. Secades JJ, Lorenzo JL. Citicoline: pharmacological and clinical review. Methods Find Exp Clin Pharmacol 2006;28:1.

19. Zani A, Cordischi L, Cananzi M, et al. Assessment of a neonatal rat model of necrotizing enterocolitis. Eur J Pediatr Surg 2008;18:423.

20. Caplan MS, Hedlund E, Adler L, et al. Role of asphyxia and feeding in a neonatal rat model of necrotizing enterocolitis. Pediatr Pathol 1994;14:1017.

21. Jilling $\mathrm{T}$, Lu J, Jackson $\mathrm{M}$, et al. Intestinal epithelial apoptosis initiates gross bowel necrosis in an experimental rat model of neonatal necrotizing enterocolitis. Pediatr Res 2004;55:622.

22. Bouadma L, Schortgen F, Ricard JD, et al. Ventilation strategy affects cytokine release after mesenteric ischemia- reperfusion in rats. Crit Care Med 2004;32:1563.

23. Sun Y, Oberley LW, Li Y. A simple method for clinical assay of superoxide dismutase. Clin Chem 1988;34:497.

24. Paglia DE, Valentine WN. Studies on the quantitative and qualitative characterization of erythrocyte glutathione peroxidase. J Lab Clin Med 1967;70:158.

25. Draper HH, Hadley M. Malondialdehyde determination as index of lipid peroxidation. Methods Enzymol 1990;186:421. 
26. Tihan DN, Erbil Y, Seven R, et al. The effect of glutamine on oxidative damage in an experimental abdominal compartment syndrome model in rats. Ulus Travma Acil Cerrahi Derg 2011;17:1.

27. Folch J, Lees M, Sloane-Stanley GH. A simple method for the isolation and purification of total lipids from animal tissues. J Biol Chem 1957;226:497.

28. Afrazi A, Sodhi CP, Richardson W, et al. New insights into the pathogenesis and treatment of necrotizing enterocolitis: toll- like receptors and beyond. Pediatr Res 2011;69:183.

29. Neu J, Walker WA. Necrotizing enterocolitis. N Engl J Med 2011;364:255.

30. Grave GD, Nelson SA, Walker WA, et al. New therapies and preventive approaches for necrotizing enterocolitis: report of a research planning workshop. Pediatr Res 2007;62:510.

31. Berman L, Moss RL. Necrotizing enterocolitis: an update. Semin Fetal Neonatal Med 2011;16:145.

32. Allen A, Leonard AJ, Sellers LA. The mucus barrier. Its role in gastroduodenal mucosal protection. J Clin Gastroenterol 1988;10:S93.

33. Braun A, Treede I, Gotthardt D, et al. Alterations of phospholipid concentration and species composition of the intestinal mucus barrier in ulcerative colitis: a clue to pathogenesis. Inflamm Bowel Dis 2009;15:1705.

34. Lichtenberger LM. The hydrophobic barrier properties of gastrointestinal mucus. Ann Rev Physiol 1995; 57:565.

35. Treede I, Braun A, Sparla R, et al. Anti-inflammatory effects of phosphatidylcholine. J Biol Chem 2007;282:27155.

36. Eros G, Kaszaki J, Czobel M, et al. Systemic phosphatidylcholine pretreatment protects canine esophageal mucosa during acute experimental biliary reflux. World J Gastroenterol 2006;12:271.

37. Demirbilek S, Aydin G, Yucesan S, et al. Polyunsaturated phosphatidylcholine lowers collagen deposition in a rat model of corrosive esophageal burn. Eur J Pediatr Surg 2002; 12:8.

38. Ehehalt $R$, Braun $A$, Karner $M$, et al. Phosphatidylcholine as a constituent in the colonic mucosal barrierdphysiological and clinical relevance. Biochim Biophys Acta 2010;1801:983.

39. Stremmel W, Merle U, Zahn A, et al. Retarded release phosphatidylcholine benefits patients with chronic active ulcerative colitis. Gut 2005;54:966.

40. Stremmel W, Ehehalt R, Autschbach F, et al.Phosphatidylcholine for steroid-refractory chronic ulcerative colitis: a randomized trial. Ann Intern Med 2007;147:603.

41. Hecht G. Innate mechanisms of epithelial host defense: spotlight on intestine. Am J Physiol 1999;277:C351.

42. Andrianifahanana M, Moniaux N, Batra SK. Regulation of mucin expression: mechanistic aspects and implications for cancer and inflammatory diseases. Biochim Biophys Acta 2006;1765:189.

43. McElroy SJ, Prince LS, Weitkamp JH, et al. Tumor necrosis factor receptor 1-dependent depletion of mucus in immature small intestine: a potential role in neonatal necrotizing enterocolitis. Am J Physiol Gastrointest Liver Physiol 2011; 301:G656.

44. Baregamian N, Song J, Bailey CE, et al. Tumor necrosis factor-alpha and apoptosis signal-regulating kinase 1 control reactive oxygen species release, mitochondrial autophagy, and c-Jun N-terminal kinase/p38 phosphorylation during necrotizing enterocolitis. Oxid Med Cell Longev 2009;2:297.

45. Tracey KJ. Physiology and immunology of the cholinergic antiinflammatory pathway. J Clin Invest 2007;117:289.

46. Johannson M, Norrgard O, Forsgren S. Presence of a marked nonneuronal cholinergic system $\mathrm{n}$ human colon: study of normal colon and colon in ulcerative colitis. Inflamm Bowel Dis 2007;13:1347.

47. Holmes-McNary MQ, Baldwin AS Jr, Zeisel SH. Opposing regulation of choline deficiency-induced apoptosis by p53 and nuclear factor jB. J Biol Chem 2001;276:41197.

48. IIcol YO, YIlmaz Z, Ulus IH. Endotoxin alters serum-free choline and phospholipid-bound cholineconcentrations, and choline administration attenuates endotoxin-induced organ injury in dogs. Shock $2005 ; 24: 288$.

49. Schneider H, Braun A, Fullekrug J, et al. Lipid based therapy for ulcerative colitis- modulation of intestinal mucus membrane phospholipids as a tool to influence inflammation. Int J Mol Sci 2010;11:4149.

50. Treede I, Braun A, Jeliaskova P, et al. TNF-a-induced up- regulation of pro-inflammatory cytokines is reduced by phosphatidylcholine in intestinal epithelial cells. BMC Gastroenterol 2009;9:53. 
51. Miller MJ, McNeill H, Mullane KM, et al. SOD prevents damage and attenuates eicosanoid release in a rabbit model of necrotizing enterocolitis. Am J Physiol 1988;255:556.

52. Crissinger KD, Grisham MB, Granger DN. Developmental biology of oxidant-producing enzymes and antioxidants in the piglet intestine. Pediatr Res 1989;25:612.

53. Ghyczy M, Torday C, Kaszaki J, et al. Oral phosphatidylcholine pretreatment decreases ischemiareperfusion-induced methane generation and the inflammatory response in the small intestine. Shock 2008;30:596.

54. Cansev M, Ilcol YO, Yilmaz MS, et al. Choline, CDP-choline or phosphocholine increases plasma glucagon in rats: involvement of the peripheral autonomic nervous system. Eur J Pharmacol 2008;589:315.

55. Ilcol YO, Cansev M, Yilmaz MS, et al. Peripheral administration of CDP-choline and its cholinergic metabolites increases serum insulin: muscarinic and nicotinic acetylcholine receptors are both involved in their actions. Neurosci Lett 2008;431:71.

56. Guo H, Liu D, Gelbard H, et al. Activated protein C prevents neuronal apoptosis via protease activated receptors 1 and 3. Neuron 2004;41:563.

57. Joyce DE, Gelbert L, Ciaccia A, et al. Gene expression profile of antithrombotic protein c defines new mechanisms modulating inflammation and apoptosis. J Biol Chem 2001; 276:11199.

58. Kumral A, Yesilırmak DC, Tugyan K, et al. Activated protein $C$ reduces intestinal injury in an experimental model of necrotizing enterocolitis. J Ped Surg 2010;45:483.

59. Fiedorowicz M, Makarewicz D, Stanczak-Brozek KI, et al. CDP-choline (citicoline) attenuates brain damage in a rat model of birth asphyxia. Acta Neurobiol Exp 2008;68:389.

60. Gellen B, Kovacs J, Nemeth L, et al. Vascular changes play a role in the pathogenesis of necrotizing enterocolitis in asphyxiated newborn pigs. Pediatr Surg Int 2003;19:380.

61. Yılmaz MS, Yalcın M, Savcı V. Cytidine 5'-diphosphocholine restores blood flow of superior mesenteric and renal arteries and prolongs survival time in haemorrhaged anaesthetized rats. Clin Exp Pharmacol Physiol 2006;33:415.

62. Savci V, Goktalay G, Cansev M, et al. Intravenously injected CDP-choline increases blood pressure and reverses hypotension in haemorrhagic shock: effect is mediated by central cholinergic activation. Eur J Pharmacol 2003;468:129. 



\section{Chapter}

\section{Evaluation of melatonin and prostaglandin E1 combination on necrotizing enterocolitis model in neonatal rats}

Published as: Cekmez F, Cetinkaya M, Tayman C, Canpolat FE, Kafa IM, Uysal S, Tunc T, Sarici SU. Evaluation of melatonin and prostaglandin E1 combination on necrotizing enterocolitis model in neonatal rats. Regul Pept 2013; 184: 121-125 


\section{Abstract}

\section{Background:}

Necrotizing enterocolitis (NEC) is one of the most common gastrointestinal emergencies in newborn infants but up to now there is no completely effective treatment for it.

\section{Objective:}

In order to show that a combination of melatonin and prostaglandins may be useful to save lives, we use newborn rat as a model of necrotizing enterocolitis to test the hypothesis of using the combination therapy might have more potential effect on mucosal cytoprotection and healing.

\section{Patients and methods:}

A total of 60 newborn pups from 5 time-mated Sprague-Dawley pregnant rats were divided equally into 5 groups as follows: NEC (subjected to NEC), NEC + Melatonin, NEC + Prostaglandin, NEC + Prostaglandin + Melatonin and control. These animals were fed with hyperosmolar formula 3 times daily and subjected to $100 \% \mathrm{CO}_{2}$ inhalation for 10 $\min ,+4{ }^{\circ} \mathrm{C}$ cold exposure for $5 \mathrm{~min}$, and $97 \% \mathrm{O}_{2}$ for $5 \mathrm{~min}$ twice daily to induce NEC. This procedure was applied to the pups for 3 days.

\section{Results:}

The macroscopic scoring, intestinal injury scoring and apoptosis index scoring were all found to be significantly lower in NEC + Prostaglandin + Melatonin group compared with NEC group. Anti-oxidant enzyme activities were significantly higher, whereas lipid peroxidation was significantly lower in NEC + Prostaglandin + Melatonin group compared with NEC group.

\section{Conclusion:}

This combination therapy showed cytoprotective and healing effects on mucosa in the intestinal tissue of rat pups in necrotizing enterocolitis model. Therefore, this therapy might also show benefit in preterm infants with NEC. After confirmation of this data by other clinical and experimental studies, it may be a novel therapeutic option for the prevention of NEC in preterm infants. 


\section{Introduction}

Necrotizing enterocolitis (NEC) is one of the most common gastrointestinal emergencies in newborn infants. Although NEC is most commonly observed in premature infants, $10 \%$ of the affected patients are born at term. Its incidence varies between 0.3 and 2.4 infants/ 1000 live births and between 7\% and 11\% among infants of less than $1500 \mathrm{~g}$. The pathophysiology of NEC remains poorly understood [1]. Several factors appear to play either a primary or a secondary role as follows: immature gut functions, impaired intestinal barrier, disturbed gastrointestinal motility, circulatory regulation, lack of some protective enzymes of cytokine-dependent injury, and undeveloped antioxidant capacity [2-4]. Intestinal ischemia, formula feeding and bacterial infections are also suggested to be the main risk factors initiating cellular injury in premature intestine [5]. Increased proinflammatory cytokines are found in intestinal samples from NEC cases, suggesting that these mediators also play a role in NEC development [1].

Melatonin activates various physiological functions causing sleep-propensity, sleep/wake rhythm, circadian rhythm, blood pressure control, immune system activity, detoxification of free radicals, protection of gastrointestinal mucosa, control of tumor growth and many others. Melatonin is known as one of the most potential anti-oxidant substances in human body and especially synthesized in gastrointestinal tract after feeding [6]. The major mechanism of melatonin on cytoprotection effect includes free radical scavenging activity and activation of cyclooxygenase-prostaglandin enzyme system [7].

Misoprostol, a prostaglandin (PG) E1 analog, is used due to its cytoprotective activity on gastrointestinal system mucosa through inhibiting acid secretion and to stimulate bicarbonate and mucus secretion [8]. In addition to its cytoprotective activity, misoprostol has also preventive effects on bacterial invasion and reduces inflammation and tissue injury [9]. Because of this reason, we hypothesized that using the combination of melatonin and prostaglandin on NEC model in newborn rats might have more potential effect on mucosal cytoprotection and healing.

In order to show that a combination of melatonin and PG may be useful to save lives, we used newborn rats as a NEC model and we aimed to test our hypothesis.

\section{Materials and methods}

\subsection{Animals and experimental design}

The project was approved by the Experimental Animal Ethics Committee at Gulhane Military Medical Academy (Ankara, Turkey) and the National Institutes of Health Guide (Washington, D.C., USA) for the Care and Use of Laboratory Animals was followed. The pregnant rats were kept in identical cages and were fed with regular laboratory chow and 
water. 60 newborn pups from 5 time-mated Sprague-Dawley pregnant rats were divided equally into 5 groups as follows: NEC (subjected to NEC), NEC + Melatonin, NEC + Prostaglandin, NEC + Prostaglandin + Melatonin and control (left with their mother to breast feed freely). Ten of them died during this period.

\subsubsection{Necrotizing enterocolitis procedure}

In the NEC (subjected to NEC), NEC + Melatonin, NEC + Prostaglandin, and NEC + Prostaglandin + Melatonin groups, based on the preventive effect of mother milk, newborn pups were immediately separated from their mothers and kept at $37^{\circ} \mathrm{C}$ in a humidified incubator. These animals were fed $0.2 \mathrm{~mL}$ of special rodent formula (15 g Similac $60 / 40$ [Ross Pediatrics, Columbus, Ohio]) prepared with $75 \mathrm{~mL}$ of puppy-canine milk replacement (Beaphar-Bogena, BV Sedel, Netherlands) 3 times daily and subjected to $100 \% \mathrm{CO}_{2}$ inhalation for $10 \mathrm{~min},+4{ }^{\circ} \mathrm{C}$ cold exposure for $5 \mathrm{~min}$, and $97 \% \mathrm{O}_{2}$ for $5 \mathrm{~min}$ twice daily to induce NEC. This procedure was applied to the pups for 3 days. The pups were weighed daily, and their weight loss or gain was recorded.

\subsubsection{Melatonin and prostaglandin E1 therapy}

We used prostaglandin E1 (Prostavasin ${ }^{\circledR}, 100$ _g amp; Schwarz- Pharma, Mannheim, Germany; 75 _g/kg intra peritoneal), melatonin (Melatonin ${ }^{\circledR}, 1$ g flacon;N-acetyl-5methoxytryptamine; Sigma Chemicals Co.; $10 \mathrm{mg} / \mathrm{kg}$ intraperitoneal).

\subsection{Biochemical analysis}

The efficacy of melatonin and prostaglandin was assessed by the tissue level of malondialdehyde (MDA) as described by Draper et al. [10], superoxide dysmutase (SOD) as described by Sun et al. [11], and glutathione peroxidase (GSH-Px) as described by Paglia and Valentine [12].

\subsection{Macroscopic scoring, histopathologic examination and apoptosis evaluation}

A macroscopic assessment of the resected gut was performed using a scoring system based on gut color, consistency and degree of dilatation. Macroscopic scoring was graded as follows: grade 0, normal; grade 1, moderately friable, patchy discoloration, and patchy dilatation; grade 2, extremely friable (jelly like), extensive discoloration, and extensive dilatation [13]. Tissue histopathologic examination and apoptosis scoring were evaluated by the same pathologist in blinded fashion. For each tissue specimen, corresponding blocks of both hematoxylin and eosin (H\&E) and transferase mediated dUTP nick end labeling (TUNEL) staining were scored. For both light microscopy and apoptosis evaluation, the distal ileum was kept in $10 \%$ neutral buffered formalin solution. Specimens were paraffin embedded, and blocks were sliced into $5-\mu \mathrm{m}$ portions and stained with H\&E. The histopathologic evaluation was graded as follows: grade 0 , normal; grade 1 (mild), separation of villous core, without other abnormalities; grade 2 
(moderate), villous core separation, submucosal edema, and epithelial sloughing; and grade 3 (severe), denudation of epithelium with loss of villous, full thickness necrosis, or perforation [14]. For apoptosis evaluation, the sections were deparaffinized and rehydrated through graded alcohols to water. The sections were then incubated with 20 $\mathrm{mg} / \mathrm{mL}$ of proteinase K (Dako, Glostrup, Den- mark) for $10 \mathrm{~min}$ at room temperature (RT). Endogenous peroxidase activity was quenched by incubation with $3 \% \mathrm{H}_{2} \mathrm{O}_{2}$ in phosphate buffer solution (PBS) (50 mmol/L sodium phosphate, $\mathrm{pH} 7.4,200 \mathrm{mmol} / \mathrm{L} \mathrm{NaCl}$ ) for $5 \mathrm{~min}$ at RT. After washing with deionized water $\left(\mathrm{dH}_{2} \mathrm{O}\right)$, the equilibration buffer was incubated for $10 \mathrm{~s}$. Enzymatic incorporation of nucleotides labeled with Working Strength TdT (ApopTag Per- oxidase in situ Apoptosis Detection Kit, S7101, Millipore, Billerica, MA, USA) were placed at $37^{\circ} \mathrm{C}$ in a humidified chamber for $60 \mathrm{~min}$. Then the sections were treated with Working Strength Stop/Wash buffer for $10 \mathrm{~min}$ at RT. The slides were washed twice in PBS. They were incubated with antidigoxigenin peroxidase at RT in a humidified chamber for $30 \mathrm{~min}$. They were washed with PBS and then they were incubated with 3,3 9-diaminobenzidine tetrahydrochloride (DAB) $(0.5 \mathrm{mg} / \mathrm{mL})$, which was used as chromogen substrate, for $6 \mathrm{~min}$ at RT in a humidified chamber. Tissue sections were lightly counterstained with $0.5 \%$ methyl green to reveal nuclei, and the slides were examined using an Axiolab microscope (Carl Zeiss, Jena, Germany). Apoptosis scoring was graded as follows: grade 0 , normal; grade 1 , apoptotic nuclei present at villous tips; grade 2 , apoptotic nuclei covering all villous tips but crypts protected; and grade 3 , transmural spread of apoptotic nuclei [15].

\section{Results}

We showed biochemical evaluations for each group in Table 1. SOD and GSH-Px levels (1965 \pm 42 vs. $1041 \pm 102 \mathrm{U} / g$ protein, $p<0.001 ; 70 \pm 2.6$ vs. $49.7 \pm 3.5 \mathrm{U} / g$ protein, $p<$ 0.001 ) were significantly higher and MDA levels (0.86 $\pm 0.1 \mathrm{vs.} 1.6 \pm 0.2 \mathrm{nmol} / \mathrm{g}$ protein, $p<0.001$ ) were significantly lower in NEC + Prostaglandin + Melatonin group comparing to NEC group.

We showed comparison of histopathologic evaluation of groups in Table 2 and Fig. 1. We found significantly lower macroscopic scoring, intestinal injury scoring (Fig. 2), and apoptosis index scoring $(0.3 \pm 0.1$ vs $1.7 \pm 0.4, p<0.001 ; 1.5 \pm 0.8$ vs. $2.6 \pm 0.5, p<0.001$ and $0.8 \pm 0.2$ vs. $2.4 \pm 0.6, p<0.001$; respectively) in NEC + Prostaglandin + Melatonin group compared with NEC group. NEC + Prostaglandin + Melatonin group had also more beneficial results in terms of biochemical and histopathologic evaluations compared with $\mathrm{NEC}+$ Melatonin and NEC + Prostaglandin groups. 
Table 1 Biochemical evaluations for each group.

\begin{tabular}{|c|c|c|c|c|c|}
\hline & $\begin{array}{l}\text { Control } \\
(n=12)\end{array}$ & $\operatorname{NEC}(n=9)$ & $\begin{array}{c}\text { NEC + Melatonin } \\
\quad(n=10)\end{array}$ & $\begin{array}{c}\text { NEC + Prostaglandin } \\
(n=9)\end{array}$ & $\begin{array}{c}\text { NEC }+ \text { Melatonin }+ \\
\text { Prostaglandin } \\
(n=10)\end{array}$ \\
\hline $\begin{array}{l}\text { MDA (nmol/g } \\
\text { protein) }\end{array}$ & $0.5 \pm 0.08$ & $1.6 \pm 0.2$ & $1.1 \pm 0.1^{*^{+\dagger}}$ & $1.3 \pm 0.1+\infty$ & $0.86 \pm 0.1 \ddagger$ \\
\hline SOD (U/g protein) & $2486 \pm 107$ & $1041 \pm 102$ & $1878 \pm 85^{*+\dagger}$ & $1774 \pm 105+\infty$ & $1965 \pm 42$ \\
\hline GSH-Px (U/g protein) & $84.2 \pm 1.9$ & $49.7 \pm 3.5$ & $65.5 \pm 1.6^{*+\dagger}$ & $62 \pm 2.7+\infty$ & $70 \pm 2.6 \ddagger$ \\
\hline
\end{tabular}

$* p<0.001$ NEC + Melatonin vs. NEC.

$+p<0.001$ NEC + Prostaglandin vs. NEC.

$\ddagger p<0.001 \mathrm{NEC}+$ Melatonin + Prostaglandin vs. NEC.

$+\dagger p<0.05 \mathrm{NEC}+$ Melatonin + Prostaglandin vs. NEC + Melatonin.

$\infty \mathrm{p}<0.05 \mathrm{NEC}+$ Melatonin + Prostaglandin vs. NEC + Prostaglandin.

Table 2 Comparison of histopathologic evaluation.

\begin{tabular}{llll}
\hline & Macroscopic scoring & Intestinal injury scoring & Apoptosis index scoring \\
\hline Control $(n=12)$ & $0 \pm 0.0$ & $0 \pm 0.0$ & $0 \pm 0.0$ \\
NEC $(n=9)$ & $1.7 \pm 0.4$ & $2.6 \pm 0.5$ & $2.4 \pm 0.6$ \\
NEC + Melatonin $(n=10)$ & $0.5 \pm 0.4$ & $7^{*}$ & $1.3 \pm 0.4^{*}+\dagger$ \\
NEC + Prostaglandin $(n=9)$ & $0.6 \pm 0.4+$ & $2.1 \pm 1+$ & $1.7 \pm 0.3+\infty$ \\
NEC + Melatonin + & $0.3 \pm 0.1 \ddagger$ & $1.5 \pm 0.8 \ddagger$ & $0.8 \pm 0.2 \ddagger$ \\
Prostaglandin $(n=10)$ & & & \\
\hline
\end{tabular}

$* p<0.001$ NEC + Melatonin vs. NEC.

$+p<0.001$ NEC + Prostaglandin vs. NEC.

$\ddagger \mathrm{p}<0.001 \mathrm{NEC}+$ Melatonin + Prostaglandin vs. NEC.

十+ $p<0.05 \mathrm{NEC}+$ Melatonin + Prostaglandin vs. NEC + Melatonin.

$\infty \mathrm{p}<0.05 \mathrm{NEC}+$ Melatonin + Prostaglandin vs. NEC + Prostaglandin 
$H \& E$

TUNEL stained

TUNEL-fluorescent

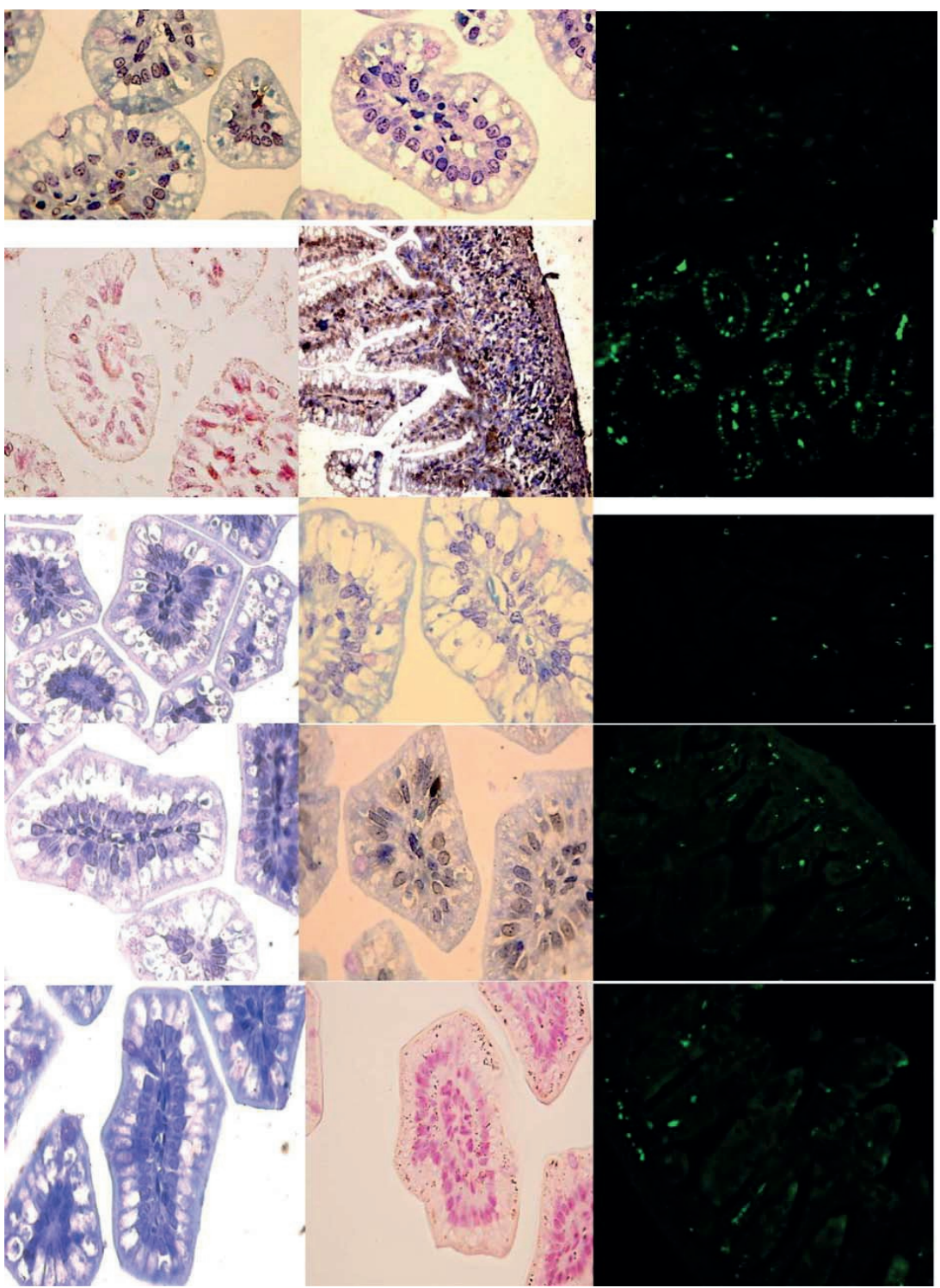

Fig. 1. Pathologic figures (first line control, second line NEC, third line melatonin, fourth line prostaglandin, fifth line combination). 


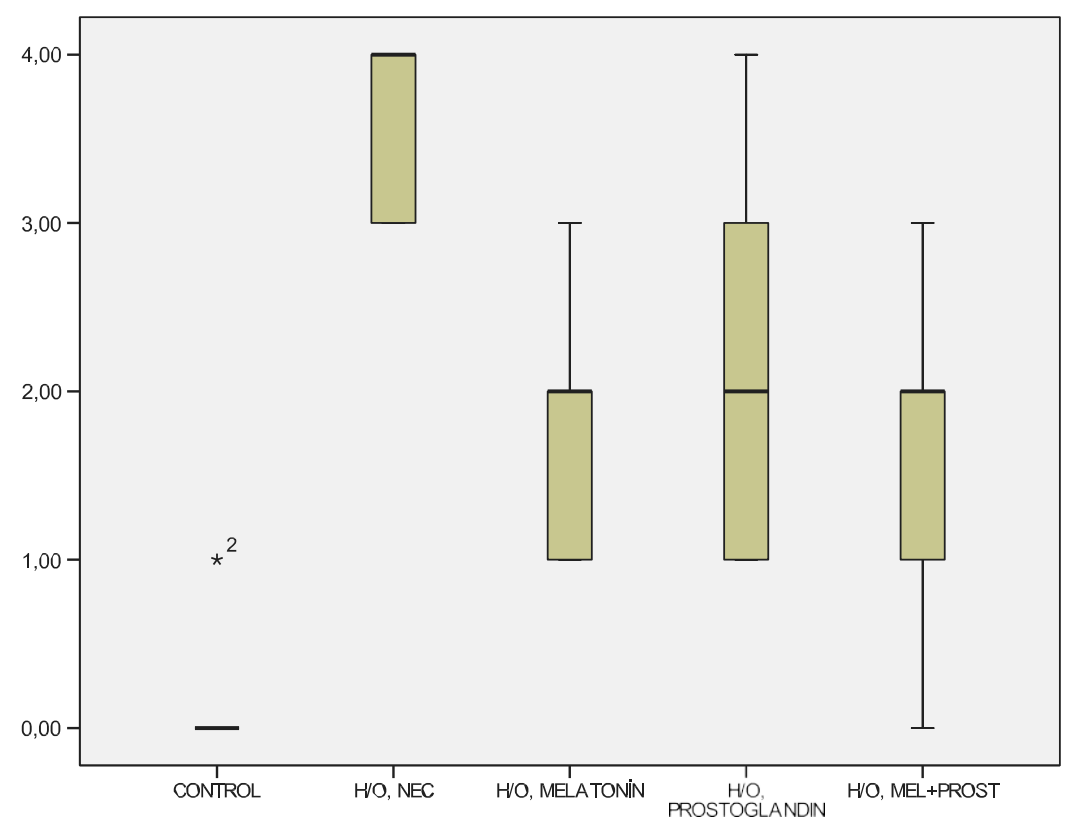

Fig. 2. Intestinal injury scores of the groups.

\section{Discussion}

In this study, we evaluated the efficacy of both single and combined therapies of melatonin and prostaglandin in a neonatal NEC rat model and we found significantly increased anti-oxidant and also decreased lipid peroxidation in combination therapy compared with the NEC, NEC + Melatonin groups and NEC + Prostaglandin groups. Macroscopic scoring, intestinal injury scoring and apoptosis index scoring were also significantly lower in combination group compared with other groups. These data suggest the use of combination therapy in preterm infants with NEC after similar experimental and clinical studies.

It is well known that besides the beneficial effect of oxygen and its derivates, they may also exert a harmful action, because of the super oxide anion radical, hydrogen peroxide and hydroxyl radicals. There are numerous endogenous and exogenous factors such as acid, pepsin, bile salts, and nonsteroidal anti-inflammatory drugs and stress factors such as ischemia, hypoxia, and infections which lead to impaired gastrointestinal mucosal barriers by acting through the production of radical oxygen species and radical nitrogen species [7,16-18].

Melatonin is known as one of the most potential antioxidant substances in human body and it is especially synthesized in gastrointestinal (GI) tract after feeding [6]. Firstly, Raikhlin and Kvetoy [19] showed that GI system produced melatonin mainly in serotonin- 
rich entero-endocrine cells (especially enterechromaffin cells). They also stated that it acted as a paracrine molecule and hormone released into the portal vein [19]. Melatonin concentration in GI system is 100-400 times higher than the blood plasma levels and this production of melatonin increases after food intake [20]. Especially in recent years, there are many studies in literature about using this antioxidant effect of melatonin in many pathological conditions. For example, Pereira reported that dietary supplementation of melatonin resulted in remarkable remission in gastroesophageal reflux disease [21]. Reiter et al. [22] showed that melatonin had an effect for scavenging oxidant substances. Kesik et al. [23] showed the healing effect of melatonin in ischemia-reperfusion model in rats [23]. In another study, Reiter et al. [22] showed that melatonin decreased the free radical formation via inhibiting the prooxidant enzymes, and activating the antioxidant enzymes; and thus it supports the antioxidant system. Melatonin sticks to the outer surface of the bi-layer lipid membrane when it comes in contact with the cell, and works as a free radical scavenger. It protects the cell and the membrane in this way $[24,25]$. In agreement with this data, we found that both melatonin and melatonin + prostaglandin increased the levels of anti-oxidant enzymes in this experimental study.

Stress factors such as ischemia, hypoxia, and infections may cause partial inactivation of cycloooxgenase-1 (COX-1) and reduce the generation of gastroprotective PGs (especially PGE1, PGE2, PGI2) that occur throughout the GI mucosa and inhibit gastric acid secretion, and increase mucosal blood flow. Expression of COX-2 may be actually increased under stress conditions due to reduction activity of COX-1, which normally exerts tonic inhibitory influence on COX-2 expression so the PG content in gastric mucosa remains low [25]. Misoprostol is an analog of prostaglandin E1. Mechanisms for the cytoprotective activity of misoprostol have still not been entirely revealed despite extensive studies. Misoprostol prevented gastric or duodenal ulcer in most of the patients using non-steroidal anti-inflammatory drugs and decreased gastrointestinal symptoms, in addition to its mucosal protective effects [26]. In our study, we found significantly higher SOD and GSH-Px levels and lower MDA levels in PG group than control and NEC groups. Macroscopic scoring, intestinal injury scoring and apoptosis index scoring were also significantly lower in PG group than in control and NEC groups. Topcu et al. [27] reported beneficial effect of misoprostol in ischemia-reperfusion induced intestinal injury in adult rats. In another study, Topsakal et al. [28] found that prostaglandin E1 and melatonin were effective for the prevention of lipid peroxidation in a spinal cord injury model in rats. Similar to these data, PG alone or especially in combination with melatonin was found as beneficial treatment options in NEC by decreasing lipid peroxidation, increasing anti-oxidant enzyme activities and ameliorating histopathological changes.

In recent years, multimodal therapeutic approach interfering at different levels of hypoxia-ischemia, inflammation and apoptosis were suggested to be more effective for preventing diseases with a multifactorial etiology such as hypoxic-ischemic encephalopathy. Cetinkaya et al. [29] reported that melatonin alone or in combination with magnesium sulfate had beneficial effect for preventing hypoxic ischemic brain injury 
in a rat model. The same group also found that both melatonin and topiramate that were administered alone or in combination were effective as neuroprotective agents [30]. Therefore, as hypoxia-ischemia is one of the major risk factors in NEC and has a similar multifactorial pathogenesis of hypoxic-ischemic brain injury, we hypothesized that the combination of two agents, melatonin, a free radical scavenger and anti-oxidant, and misoprostol, mucosal gastroprotective, would have show protective effect against NEC development and preformed this experimental study. In agreement with these studies, our study showed that the combination therapy provided significantly more beneficial effects for the prevention of NEC compared with the single administration of melatonin and misoprostol. Therefore, we suggest that the combined therapies that have different actions in the pathogenesis of NEC may be used in infants with NEC after confirmation of our data. In conclusion, to our best of knowledge, this is the first study that showed a significant protective effect of combination therapy of misoprostol and melatonin, two agents acting on different stages of NEC. These data may also be used as a reference point in the clinical treatment of preterm infants with NEC.

However, to make such a recommendation, much more experimental and clinical studies are needed.

\section{Disclosure}

The authors do not have any conflict of interest to disclose. 


\section{References}

1. Lin PW, Stoll BJ. Necrotising enterocolitis. Lancet 2006;368:1271-83.

2. Ryder RW, Shelton JD, Guinan ME. Necrotizing enterocolitis: a prospective multi- center investigation. Am J Epidemiol 1980;112:113-23.

3. Fox TP, Godavitarne C. What really causes necrotising enterocolitis? ISRN Gastroenterol 2012;2012:9. http://dx.doi.org/10.5402/2012/628317.

4. Hsueh W, Caplan MS, Qu XW, et al. Neonatal necrotizing enterocolitis: clinical considerations and pathogenetic concepts. Pediatr Dev Pathol 2003;6:6-23.

5. Covert RF, Neu J, Elliott MJ, et al. Factors associated with age of onset of necrotiz- ing enterocolitis. Am J Perinatol 1989;6:455-60.

6. Reppert SM, Klein DC. Transport of maternal[3H]melatonin to suckling rats and the fate of [3H]melatonin in the neonatal rat. Endocrinology Feb. 1978;102(2):582-8.

7. Konturek SJ, Konturek PC, Brzozowska I, Pawlik M, Sliwowski Z, Cześnikiewicz-Guzik

8. M, Kwiecień S, Brzozowski T, Bubenik GA, Pawlik WW. Localization and biological activities of melatonin in intact and diseased gastrointestinal tract (GIT).J Physiol Pharmacol Sep. 2007;58(3):381-405 [Review].

9. Smedfors B, Johansson C. Stimulation of duodenal bicarbonate secretion by miso- prostol. Dig Dis Sci 1986;3:S96-S100.

10. Davies NM, Longstreth J, Jamali F. Misoprostol therapeutics revisited. Pharmaco- therapy 2001;21:60-73.

11. Draper HH. HadleyM: malondialdehyde determination as index of lipid peroxida- tion. Methods Enzymol 1990;186:421-31.

12. Sun K, Cao S, Pei L, Matsuura A, Xiang L, Qi J. A Steroidal Saponin from Ophiopogon japonicus Extends the Lifespan of Yeast via the Pathway Involved in SOD and UTH1. Int J Mol Sci 2013 Feb 25;14(3):4461-75.

13. Valentine WN, Paglia DE. Syndromes with increased red cell glutathione (GSH). Hemoglobin 1980;4(56):799-804.

14. Zani A, Cordischi L, Cananzi M, De Coppi P, Smith VV, Eaton S, Pierro A. Assess- ment of a neonatal rat model of necrotizing enterocolitis. Eur J Pediatr Surg 2008;18:423-6.

15. Nadler EP, Dickinson E, Knisely A, et al. Expression of inducible nitric oxide synthase and interleukin-12 in experimental necrotizing enterocolitis. J Surg Res 2000;92:71-7.

16. Jilling $\mathrm{T}$, Lu J, Jackson $\mathrm{M}$, et al. Intestinal epithelial apoptosis initiates gross bowel necrosis in an experimental rat model of neonatal necrotizing enterocolitis. Pediatr Res 2004;55:622-9.

17. Bandyopadhyay D, Chattopadhyay A. Reactive oxygen species-induced gastric ulceration: protection by melatonin.Curr Med Chem 2006;13(10):1187-202 [Review].

18. Kehrer JP, Smith CV. In: Frei B, editor. Natural antioxidants in human health and disease. San Diego: Academica Pres; 1994. p. 25.

19. Sies H. Strategies of antioxidant defense. Eur J Biochem 1993;215:213-9.

20. Raikhlin NT, Kvetnoy IM. Melatonin and enterochromaffin cells. Acta Histochem 1976;55:19-24.

21. Bubenik GA, Pang SF, Cockshut JR, Smith PS, Grovum LW, Friendship RM, Hacker RR. Circadian variation of portal, arterial and venous blood levels of melatonin in pigs and its relationship to food intake and sleep. J Pineal Res Jan. 2000;28(1):9-15.

22. de Souza Pereira R. Regression of gastroesophageal reflux disease symptoms using dietary supplemantation with melatonin, vitamins and amino acids: com- parison with omeprazole. J Pineal Res 2006;41:195-200.

23. Reiter RJ, Tan DX, Jou MJ, Flores LJ, Reiter RJ. Biogenic amines in the reduction of ox-idative stress: melatonin and its metabolites. Neuro Endocrinol Lett 2008;29:391-8.

24. Kesik V, Guven A, Vurucu S, Tunc T, Uysal B, Gundogdu G, Oztas E, Korkmaz A. Melatonin and1400 W ameliorate both intestinal and remote organ injury follow- ing mesenteric ischemia/reperfusion. J Surg Res 2009;157:97-105.

25. Reiter RJ, Tan DX. Melatonin: a novel protective agent against oxidative injury of the ischemic/reperfused heart. Cardiovasc Res 2003;58:10-9. 


\section{Chapter 5}

26. Reiter RJ, Tan DX, Osuna C, Gitto E. Actions of melatonin in the reduction of oxida- tive stress. A review. J Biomed Sci 2000;7:444-58.

27. Graham DY, Agrawal NM, Roth SH. Prevention of NSAID induced gastric ulcer with misoprostol: multicentre double-blind, placebo controlled trial. Lancet 1988;2: 1277-80.

28. Topcu I, Vatansever S, Var A, Cavus Z, Cilaker S, Sakarya M. The effect of misopros- tol, a prostaglandin E1 analog, on apoptosis in ischemia-reperfusion-induced in- testinal injury.Acta Histochem 2007;109(4):3229 [Epub 2007 Apr 12].

29. Topsakal C, Kilic N, Ozveren F, Akdemir I, Kaplan M, Tiftikci M, Gursu F. Effects of prostaglandin E1, melatonin, and oxytetracycline on lipid peroxidation, antioxidant defense system, paraoxonase (PON1) activities, and homocysteine levels in an ani- mal model of spinal cord injury. Spine (Phila Pa 1976) Aug. 1 2003;28(15):1643-52.

30. Cetinkaya M, Alkan T, Ozyener F, Kafa IM, Kurt MA, Koksal N. Possible neuroprotective effects of magnesium sulfate and melatonin as pre- and post-treatment in a neonatal hypoxic-ischemic rat model. Neonatology 2011;99(4):302-10.

31. Ozyener F, Cetinkaya M, Alkan T, Goren B, Kafa IM, Kurt MA, Koksal N. Neuroprotective effects of melatonin administered alone or in combination with topiramate in neonatal hypoxic-ischemic rat model. Restor Neurol Neurosci 2012;30(5):435-44. 


\section{Chapter}

\section{Neuroprotective effects of uridine in a rat model of neonatal hypoxic-ischemic encephalopathy}

Published as: Cansev M, Minbay Z, Goren B, Yaylagul EO, Cetinkaya M, Koksal N, Alkan T. Neuroprotective effects of uridine in a rat model of neonatal hypoxic-ischemic encephalopathy. Neurosci Lett 2013; 542: 65-70. 


\section{Abstract}

Neonatal hypoxic-ischemic encephalopathy (HIE) is a major cause of neurological disability requiring newer therapeutic strategies. Uridine is the principal circulating pyrimidine in humans and a substrate for nucleotides and membrane phospholipids. The objective of this study was to investigate the effects of uridine in a neonatal rat model of HIE. Rat pups subjected to hypoxic-ischemic insult on postnatal day 7 were injected intraperitoneally with either saline or uridine $(100,300$ or $500 \mathrm{mg} / \mathrm{kg}$ ) for three consecutive days and brains were collected for evaluation of brain infarct volume and apoptosis. Compared with Control group, uridine at 300 and $500 \mathrm{mg} / \mathrm{kg}$ doses significantly reduced percent infarct volume, TUNEL (+) cell ratio and active Caspase-3 immunoreactivity in the cortex, as well as in CA1 and CA3 regions of the hippocampus. Uridine (300 and $500 \mathrm{mg} / \mathrm{kg}$ ) also decreased active Caspase-3 expression in the ipsilateral hemisphere. These data indicate that uridine dose-dependently reduces brain injury in a rat model of neonatal HIE by decreasing apoptosis. 


\section{Introduction}

Hypoxic-ischemic ( $\mathrm{HI}$ ) insult in newborns causes brain injury with significant consequences including motor and cognitive deficits and seizures [32]. Every 2 newborns per 1000 term births are affected by neonatal hypoxic-ischemic encephalopathy (HIE) [17] which causes death and neurological morbidities [29]. Although many strategies have been proposed in treatment of $\mathrm{HIE}$, newer approaches are required to ameliorate the severity of brain pathology, and reduce mortality and morbidity.

Uridine is the principal circulating pyrimidine nucleoside in humans [4,35] and a constituent of breast milk [26]. It is also a precursor of brain membrane phosphatides via the Kennedy pathway [16] as a building block of uridine-5'-triphosphate (UTP). Uridine administration enhances cytidine-5'-diphosphocholine (CDP-choline) levels in PC12 cells [23], striatal slices [27] and rodent brains [8]. CDP-choline has been reported to exhibit beneficial effects in experimental and clinical studies of stroke and other neurodegenerative states [1] including HIE [13].

Uridine nucleotides (UTP and uridine-5'-diphosphate [UDP]) are ligands to P2Y receptors (P2Y2, P2Y4 and P2Y6), stimulation of which provides neuroprotection by reducing apoptosis $[5,18]$. Uridine administration leads to enhancement in UTP levels in PC12 cells [22] and rodent brains [8].

Since uridine is a precursor to uridine nucleotides and CDP-choline, we aimed to investigate the effects of uridine administration in a rat model of neonatal HIE. Our data show for the first time that intraperitoneal (i.p.) uridine administration for 3 consecutive days following $\mathrm{HI}$ brain insult reduces infarct volume, TUNEL (+) cell ratio and active Caspase- 3 expression in the cortex as well as CA1 and CA3 regions of the hippocampus in newborn rats.

\section{Materials and methods}

Experimental protocols were approved by the Animal Care and Use Committee of Uludag University, Bursa, Turkey (2009-07/4), and the experiments conformed to the National Institutes of Health Guide for the Care and Use of Laboratory Animals (NIH Publications No. 80-23) revised 1996 and EC Directive 86/609/EEC.

Model for perinatal HI brain injury was adapted from Vanucci and Vanucci [30]. A total of 51 pups were born to 5 dated pregnant Sprague-Dawley rats which were housed in individual cages with free access to food and water with $12 \mathrm{~h} \mathrm{light/dark}$ cycle. The day of birth was considered postnatal day 0 (PNDO) and experiments were initiated on day 7 (PND7), at which day the brain maturation of rats coincides with that of 32- to 34-weekold human [31]. 


\subsection{Induction of HI brain injury}

Out of the initial 51 pups, 8 pups were excluded due to low weight on PND7 and 3 pups were excluded due to bleeding after surgery. Forty rat pups of either sex $(55 \%$ males [ $n=$ 22] and $45 \%$ females [ $n=18]$ ) weighing $12-16 \mathrm{~g}$ on PND7 were used in the experiments. Under isoflurane anesthesia, right common carotid arteries of pups were ligated and coagulated. Following a 2-h period of nesting, three pups from each dam were placed into chambers partially submerged in a $37^{\circ} \mathrm{C}$ water bath and exposed to hypoxia ( $8 \%$ oxygen balanced with nitrogen mixture) for $150 \mathrm{~min}$ (Fig. 1A). Hypoxic chambers were attached to an anesthesia device (AMS Minor 612, GMS Generra Medikal, Ankara, Turkey) and the gas flow was monitored using a gas flow analyzer (VT-PLUS HF, Fluke Biomedical, Everett, WA) before each experiment.

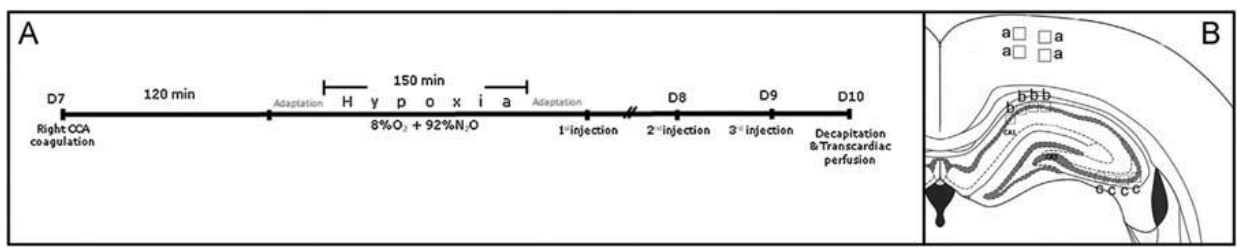

Fig. 1. Schematic diagrams of the experimental procedure $(A)$ and the areas utilized for determining TUNEL $(+)$ cell ratio and Caspase-3 immunoreactivity (B). "a", count area in cortex; "b" count area in CA1 region; "c" count area in CA3 region.

\subsection{Treatment groups}

PND7 rats $(n=32)$ were divided randomly, ensuring that all treatment groups were represented by pups from each dam, into 4 groups and injected i.p. with the following: saline (0.9\% NaCl,0.1 ml [Control group]), uridine (100 mg/kg [U100 group]), uridine (300 $\mathrm{mg} / \mathrm{kg}$ [U300 group]), and uridine (500 mg/kg [U500 group]) (dissolved in saline and injected $0.1 \mathrm{ml}$ ) (Fig. 1A). No behavioral changes were observed after $\mathrm{HI}$ insult or uridine treatments. Sham operation by exposing the common carotid artery without ligation and hypoxia was performed on another set of rat pups $(n=8)$. The dose range of uridine used in the present study has been selected in the light of a previous report which tested CDPcholine [13] in a neonatal rat model of HIE, as well as our preliminary observations with $300 \mathrm{mg} / \mathrm{kg}$ uridine with three consecutive injections.

\subsection{Brain tissue procurement}

Following completion of experiments, pups were divided into 2 groups on PND10; under deep anesthesia, pups were either decapitated and brain tissues were removed $(n=20)$ or sacrificed following transcardiac perfusion with $4 \%$ paraformaldehyde dissolved in phosphate-buffered saline (PBS) $(\mathrm{pH} 7.4)(n=20)$ for western blotting or immunohistochemistry, respectively. 


\subsection{Measurement of brain infarct volume}

One in every five $20 \mu \mathrm{m}$-thick brain sections selected with a defined range was stained with $0.5 \%$ cresyl violet (Acros Organics, Geel, Belgium) in order to evaluate brain infarct volume for each pup using images captured at a magnification of $1.25 \times$ (Fig. 2). Volume fraction of the infarct (corresponding to percent infarct volume [12]) in ipsilateral hemisphere was estimated by Cavalieri principle by a blinded investigator using a combined point-counting grid as described previously $[9,12,14]$.

\subsection{In situ cell death detection}

DNA fragmentation was detected using an in situ cell death detection POD kit (Roche Molecular Biochemicals, 11684817910, Germany) for TUNEL (terminal deoxynucleotidyl transferase (TdT)-mediated deoxyuridine triphosphate (dUTP)-biotin nick end-labeling) technique in cryosections. Slides were counter- stained with Harris's hematoxylin (SigmaAldrich, St. Louis, MO).

TUNEL $(+)$ cell count was performed on sections from four different levels in rostrocaudal plane of cortex as well as CA1 and CA3 areas of the hippocampus that corresponded approximately to panels 11-14 according to the Atlas of Prenatal Rat Brain Development [2].

Images with size of $4080 \times 3072$ square pixels were captured with CCD camera (Olympus DP71 CCD color camera, 1.5 million pixel) attached to a light microscope at 100x magnification (Olympus BX50). Four areas in cortex and regions of the hippocampus were hemilaterally analyzed (Fig. 1B). After top of the section of the selected microscopic field was found, eight consecutive focal planes with a distance of $2 \mu \mathrm{m}$ were scanned between the top and the bottom surfaces. Images of focal planes were superimposed prior to counting the nuclei and percent TUNEL $(+)$ cells over all cells were calculated for each pup and data were presented as TUNEL (+) cell ratio (Fig. 3).

In addition, four different coronal sections adjacent to those utilized for TUNEL staining were chosen for active Caspase-3 immunohistochemistry. Sections were labeled with rabbit anti-cleaved Caspase-3 (1:200; Asp-175, Cell Signaling Technology Inc., Danvers, $\mathrm{MA}$ ) and incubated with an appropriate secondary antibody (biotinylated anti-rabbit lgG, Jackson Immuno Research Laboratories Inc., West Grove, PA) before treatment with avidine biotin complex ( $A B C$ Vectastain Elite $A B C$ Kit, Vector Laboratories Inc., Burlingame, $C A$ ) and visualization using DAB substrate.

The number of Caspase- 3 positive cells was counted by a 10,000 $\mu \mathrm{m}^{2}$-sized counting frame that was viewed through a 100x magnification at captured images (Fig. 4) repeating the steps performed for TUNEL $(+)$ cell counts and cell counts of 4 sections were averaged for each region in each pup. 


\subsection{Active Caspase-3 protein expression}

Ipsilateral hemispheres were homogenized in ice-cold PBS ( $\mathrm{pH}$ 7.4). Equal amounts of protein were loaded and separated using SDS-PAGE (4-20\%; Bio-Rad, Hercules, CA). Each gel contained homogenates of four individual pups at each treatment group. Proteins were transferred onto membranes which were then incubated overnight with the primary antibody against active Caspase-3 (1:1000, Abcam, Cambridge, MA) and then with the appropriate peroxidase-linked secondary antibody (1:5000, GE Healthcare, Waukesha, WI). Protein-antibody complexes were visualized using the enhancedchemiluminescence system (GE Healthcare, Waukesha, WI) and developed on Kodak XAR films which were digitized using a scanner with a transparency adapter (UMAX Technologies, Freemont, CA). Immunoreactive bands were compared densitometrically using the NIH Image-J program. Areas under the absorbance curve were expressed as arbitrary units and normalized as percentages of control samples. Same blots were stripped and incubated with $\beta$-tubulin (1:1000, Abcam, Cambridge, MA), used as a loading control.

\subsection{Statistics}

Statistical analyses were performed using Sigma Plot 12.0 soft- ware. Data were expressed as mean \pm standard error of means (SEM). Difference between groups was determined by one-way ANOVA followed by post hoc Tukey test. $p<0.05$ was considered statistically significant.

\section{Results}

\subsection{Effect of uridine treatment on brain infarct volume}

Percent infarct volume in ipsilateral hemispheres of Control pups ( $66 \pm 3.8 \%)$ was reduced in U300 or U500 groups to $34.1 \pm 7.9 \%$ ( $p<0.05)$ or $32.7 \pm 3.5 \%(p<0.05)$, respectively (Fig. 2, lower right panel). We also detected $20 \pm 0.6 \%$ and $18 \pm 0.4 \%$ infarct volume in non-ischemic hemispheres of pups in Control and U100 groups, respectively, while no infarction was observed in those in the U300 and U500 groups (data not shown). 

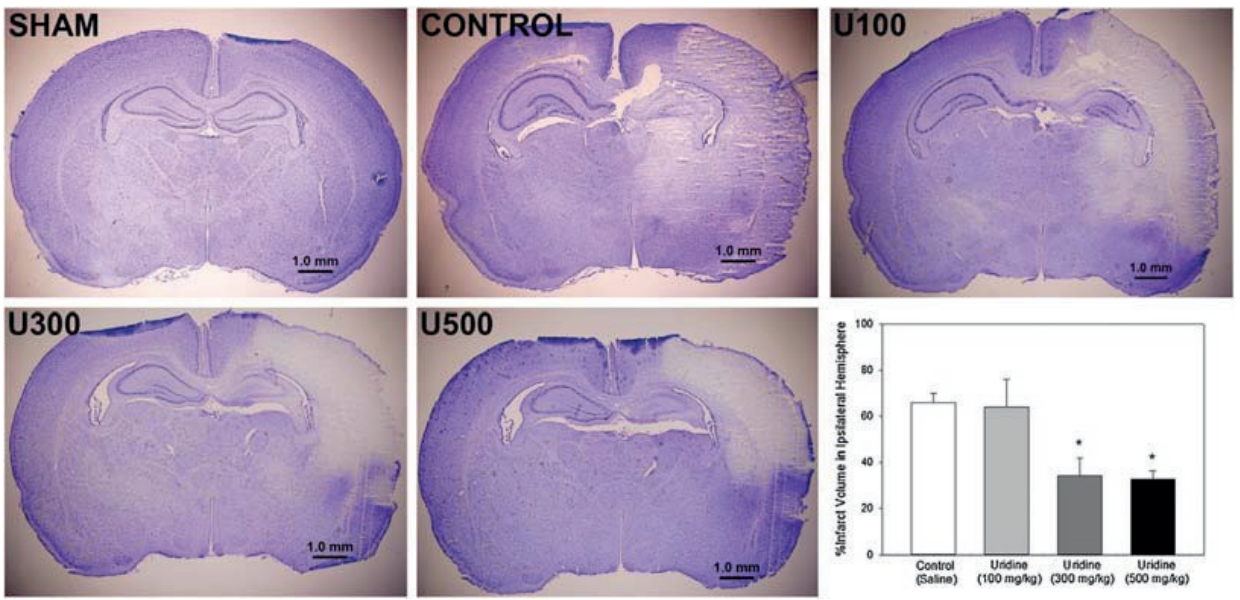

Fig. 2. Representative images of coronal sections stained with Cresyl Violet indicating brain infarct volume in sham-operated, control and uridine-treated pups (upper panels plus lower left and middle panel) and graph depicting percent infarct volume in ipsilateral hemisphere (lower right panel). ${ }^{*} p<0.05$ compared with Control group. U100, U300 and U500 represent i.p. uridine treatments at 100, 300 and $500 \mathrm{mg} / \mathrm{kg}$, respectively.

\subsection{Effect of uridine treatment on TUNEL (+) cell ratio}

Compared with the Control group, TUNEL $(+)$ cell ratio was decreased significantly in U300 or U500 groups in the cortex (by $27.1 \%$ [ $<0.05$ ] or by $32.6 \%$ [ $<0.05$ ], respectively), as well as CA1 (by $39 \%$ [ $<0.001$ ] or by $39.6 \%$ [ $<0.001]$, respectively) and CA3 (by $31.8 \%[p<0.05$ ] or by $32.5 \%$ [p $<0.05]$, respectively) regions of the hippocampus (Fig. 3B).

\subsection{Effects of uridine treatment on active Caspase-3 immunoreactivity}

Number of active Caspase-3 immunoreactive cells was reduced in U300 or U500 groups in the cortex (by 20.9 [p < 0.001] or by 22 [p < 0.001], respectively), as well as CA1 (by 21.7 [p $<0.001$ ] or by 23.8 [p < 0.001], respectively) and CA3 (by 9.8 [p < 0.001] or by 12 [p < $0.001]$, respectively) regions of the hippocampus compared with Control group (Fig. 4B). 

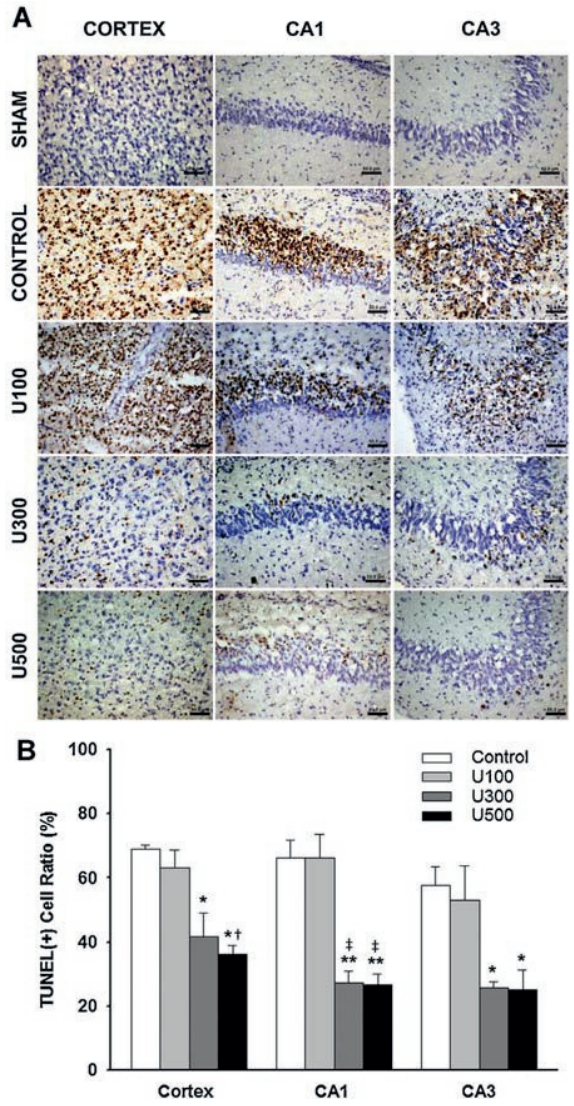

Fig. 3. Representative images of TUNEL staining in the cortex, as well as CA1 and CA3 regions of the hippocampus (A) and graph depicting TUNEL (+) cell ratio (B)

${ }^{*} p<0.05$ and ${ }^{* *} p<0.001$ compared to values obtained in Control group; $\uparrow p<0.05$

and $\ddagger p<0.001$ compared to values obtained in U100 group.

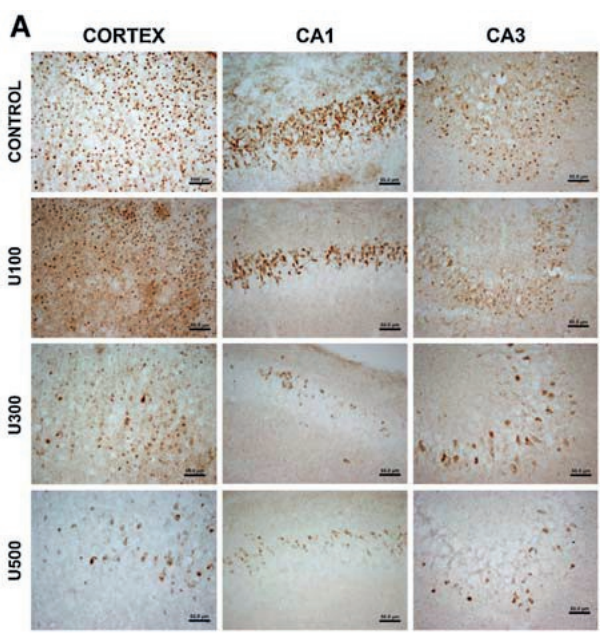

B

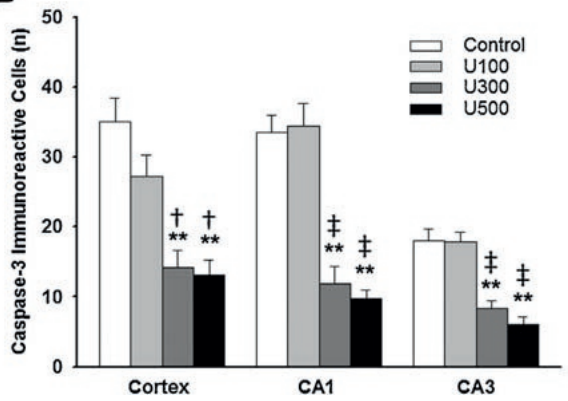

Fig. 4. Representative images of Caspase-3 immunoreactivity in the cortex, as well as CA1 and CA3 regions of the hippocampus (A) and graph depicting Caspase-3 immunoreactive cell count (B). $* * p<0.001$ compared to values obtained in Control group; $+p<0.05$ and $\ddagger p<0.001$ compared to values obtained in U100 group.

\subsection{Effects of uridine treatment on active Caspase-3 protein levels}

Compared with Control group, active Caspase-3 levels in ipsilateral hemispheres were decreased in U300 (by 22.5\%; $\mathrm{p}<0.05$ ) or U500 (by 32.5\%; $\mathrm{p}<0.05$ ) group, but not U100 group (Fig. 5). Levels of $\beta$-tubulin did not change indicating equal protein load. 


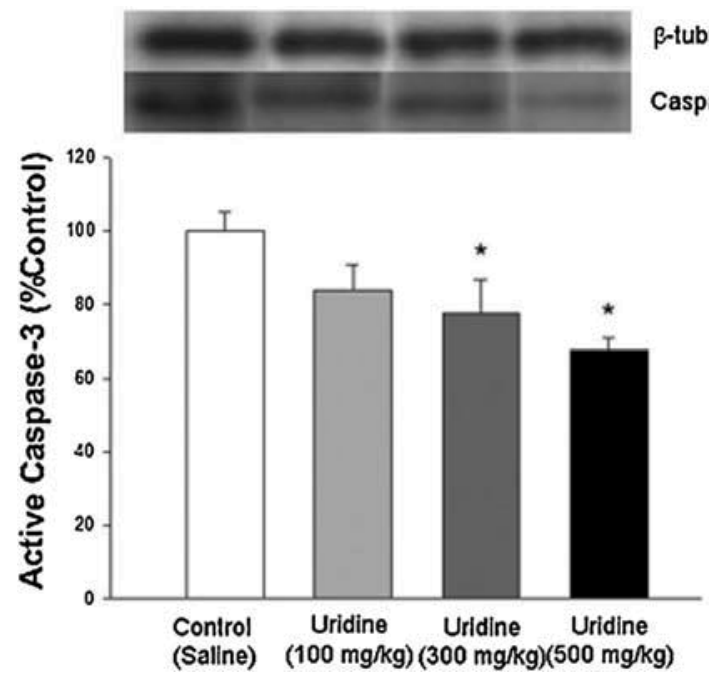

Fig. 5. Graph depicting active Caspase-3 expression as percentage of control values. Active Caspase-3 was investigated by running homogenates of ipsilateral hemispheres from four individual pups at each treatment group. $\beta$-Tubulin was used as protein loading control. ${ }^{*} p<0.05$ compared with Control.

\section{Discussion}

Our data show, for the first time, that parenteral uridine administration for three consecutive days dose-dependently reduces percent brain infarct volume, TUNEL (+) cell ratio as well as active Caspase- 3 immunoreactive cell counts and protein levels in a rat model of HIE. These data suggest that uridine might ameliorate brain lesion in newborns with $\mathrm{HI}$ insult.

Neonatal brain injury due to HIE remains a great challenge due to causing brain injury leading to motor and cognitive deficits, and seizures [32]. Many strategies including erythropoietin administration [36] and therapeutic hypothermia [25] have been proposed for the management of HIE. However, these strategies have several complications; erythropoietin may lead to such conditions as retinopathy of prematurity, hypertension, coagulation and hemangioma, and cause detrimental effects for normal development [36]. In addition, mild hypothermia which could be used within strict protocols and appropriate training on correct diagnosis in only neonatal intensive care units [15], seems efficient in only some asphyxiated newborns, but not in all [33]. Therefore, safer and more accessible approaches are required to reduce the severity of brain pathology in newborns with HIE.

Uridine is the principal circulating pyrimidine nucleoside in humans $[4,35]$ and a constituent of breast milk [26]. Uridine also serves as a precursor of membrane phosphatides and exogenous administration of uridine enhances CDP-choline levels in PC12 cells [23], striatal slices [27] and rodent brains [8]. Chronic supplementation with 
uridine-5-monophosphate (UMP) increases levels of phospholipids, synaptic proteins and leads to synaptogenesis in adult [24,34] and newborn rodents [6]. Evidence that uridine might confer benefit under degenerative conditions has been provided by in vitro glucose deprivation studies $[10,11]$ as well as in vivo rat model of Parkinson's [7].

Therefore, we tested uridine in a rat model of HIE. Our results indicate that i.p. uridine administration for three consecutive days after the onset of $\mathrm{HI}$ insult in PND7 pups reduced percent brain infarct volume in a dose-dependent manner. Uridine treatment at 300 and $500 \mathrm{mg} / \mathrm{kg}$ doses also ameliorated the infarction observed in Control and U100 groups in the non-ischemic contralateral hemisphere, suggesting that uridine is effective against the lesion caused by hypoxia alone. In addition, uridine also dose-dependently decreased TUNEL $(+)$ cell ratio in the cortex, as well as CA1 and CA3 regions of hippocampus indicating that uridine may limit the apoptotic process in newborns with HIE. This suggestion was further confirmed by significantly reduced expression and levels of active Caspase-3, an indicator of apoptosis, by uridine administration.

The mechanism(s) by which uridine ameliorate brain injury and reduce apoptosis in $\mathrm{HI}$ insult remain to be determined. Uridine is a precursor to CDP-choline which has proven beneficial in in vitro models of neurodegeneration [20] and has long been tested in stroke and other neurodegenerative states in experimental animals and humans [1]. CDPcholine exhibits cerebroprotection in a rat model of HIE through a reduction in active Caspase-3 levels [13] which is in agreement with our present findings. In addition, uridine is a precursor to UTP and UDP, pyrimidine nucleotides that are formed through phosphorylation of uridine. UTP and UDP are ligands to various P2Y receptors (P2Y2, P2Y4 and P2Y6), stimulation of which provides neuroprotection by several mechanisms including reduction of apoptosis $[5,18]$ involving intracellular signaling molecules including ERK and Akt $[3,5]$. It could also be speculated that uridine's cerebroprotection involves hypothermia, as has been demonstrated previously [21]. It is also possible that the cerebroprotective effects of uridine observed in the present study might have arisen from the action of uridine phosphorylase, which provided protection from glucose deprivation-induced death of immunostimulated astrocytes [10] and cortical neurons [11] and have involved both neurons and astrocytes. However, precise mechanism(s) of uridine action in this model needs further investigation. 


\section{Conclusions}

In conclusion, uridine administration dose-dependently reduces brain infarct volume, TUNEL (+) cell ratio and Caspase-3 levels in newborn rats subjected to $\mathrm{HI}$ insult. To the best of our knowledge, our study is the first to report beneficial effects of uridine on brain injury and apoptosis in a rat model of HIE. Previous human studies demonstrated that uridine can be tolerated at least up to $10 \mathrm{~g} / \mathrm{m}^{2}$ for a single oral dose [28] and for intravenous injection [19]. Hence, uridine might confer benefit as an easily accessible, safe and efficient therapeutic agent in human newborns with HIE.

\section{Conflict of interest}

The authors state no conflict of interest.

\section{Acknowledgement}

This study was supported by Uludag University Scientific Research Projects grant number: T/U 2009-2. 


\section{References}

1. R.M. Adibhatla, J.F. Hatcher, Cytidine 51 -diphosphocholine (CDP-Choline) in stroke and other CNS disorders, Neurochem. Res. 30 (2005) 15-23.

2. J. Altman, S.A. Bayer, Atlas of Prenatal Rat Brain Development, CRC Press, Boca Raton, FL, 1995.

3. D.B. Arthur, S. Georgi, K. Akassoglou, P.A. Insel, Inhibition of apoptosis by P2Y2 receptor activation: novel pathways for neuronal survival, J. Neurosci. 26 (2006) 3798-3804.

4. M. Cansev, Uridine and cytidine in the brain: their transport and utilization, Brain Res. Rev. 52 (2006) 389397.

5. M. Cansev, Involvement of uridine-nucleotide-stimulated P2Y receptors in neuronal growth and function, Cent. Nerv. Sys. Agents Med. Chem. 7 (2007) 223-229.

6. M. Cansev, G. Marzloff, T. Sakamoto, I.H. Ulus, R.J. Wurtman, Giving uridine and/or docosahexaenoic acid orally to rat dams during gestation and nurs- ing increases synaptic elements in brains of weanling pups, Dev. Neurosci. 31 (2009) 181-192.

7. M. Cansev, I.H. Ulus, L. Wang, T.J. Maher, R.J. Wurtman, Restorative effects of uri- dine plus docosahexaenoic acid in a rat model of Parkinson's disease, Neurosci. Res. 62 (2008) 206-209.

8. M. Cansev, C.J. Watkins, E.M. van der Beek, R.J. Wurtman, Oral Uridine 51 monophosphate (UMP) increases brain CDP-choline levels in gerbils, Brain Res. 1058 (2005) 101-108.

9. J. Chia, A. Baddeley, Accuracy of estimates of volume fraction, Image Anal. Stereol. 19 (2000) 199-204.

10. J.W. Choi, B.K. Yoo, C.Y. Shin, M.K. Ryu, J.H. Ryu, M.H. el Kouni, J.C. Lee, W.K. Kim, K.H. Ko, Uridine prevents the glucose deprivation-induced death of immunos- timulated astrocytes via the action of uridine phosphorylase, Neurosci. Res. 56 (2006) 111-118.

11. J.W. Choi, C.Y. Shin, M.S. Choi, S.Y. Yoon, J.H. Ryu, J.C. Lee, W.K. Kim, M.H. el Kouni, K.H. Ko, Uridine protects cortical neurons from glucose deprivation- induced death: possible role of uridine phosphorylase, J. Neurotrauma 25 (2008) 695-707.

12. N. Ekinci, N. Acer, A. Akkaya, S. Sankur, T. Kabadayi, B. Sahin, Volumetric evalu- ation of the relations among the cerebrum, cerebellum and brain stem in young subjects: a combination of stereology and magnetic resonance imaging, Surg. Radiol. Anat. 30 (2008) 489-494.

13. M. Fiedorowicz, D. Makarewicz, K.I. Stan' czak-Mrozek, P. Grieb, CDP-choline (citicoline) attenuates brain damage in a rat model of birth asphyxia, Acta Neurobiol. Exp. 68 (2008) 389-397.

14. H.J. Gundersen, T.F. Bendtsen, L. Korbo, N. Marcussen, A. Møller, K. Nielsen,

15. J.R. Nyengaard, B. Pakkenberg, F.B. Sørensen, A. Vesterby, Some new, simple and efficient stereological methods and their use in pathological research and diagnosis, APMIS 96 (1988) 379-394

16. S.E. Jacobs, C.J. Morley, T.E. Inder, M.J. Stewart, K.R. Smith, P.J. McNamara, I.M. Wright, H.M. Kirpalani, B.A. Darlow, L.W. Doyle, Infant Cooling Evaluation Col- laboration, Whole-body hypothermia for term and near-term newborns with hypoxic-ischemic encephalopathy: a randomized controlled trial, Arch. Pedi- atr. Adolesc. Med. 165 (2011) 692-700.

17. E.M. Kennedy, S.B. Weiss, The function of cytidine coenzymes in the biosynthe- sis of phospholipids, J. Biol. Chem. 222 (1956) 193-214.

18. J.J. Kurinczuk, M. White-Koning, N. Badawi, Epidemiology of neonatal encephalopathy and hypoxicischaemic encephalopathy, Early Hum. Dev. 86 (2010) 329-338.

19. D. Lecca, S. Ceruti, Uracil nucleotides: from metabolic intermediates to neuroprotection and neuroinflammation, Biochem. Pharmacol. 75 (2008) 1869-1881.

20. Leyva, C.J. van Groeningen, I. Kraal, H. Gall, G.J. Peters, J. Lankelma, H.M. Pinedo, Phase I and pharmacokinetic studies of high-dose uridine intended for rescue from 5-fluorouracil toxicity, Cancer Res. 44 (1984) 5928-5933.

21. Mir, J. Clotet, R. Aledo, N. Durany, J. Argemi, R. Lozano, J. Cervos-Navarro, N. Casals, CDP-choline prevents glutamate-mediated cell death in cerebellar granule neurons, J. Mol. Neurosci. 20 (2003) 53-60. 
22. G.J. Peters, C.J. van Groeningen, E.J. Laurensse, J. Lankelma, A. Leyva, H.M. Pinedo, Uridine-induced hypothermia in mice and rats in relation to plasma and tissue levels of uridine and its metabolites, Cancer Chemother. Pharmacol. 20 (1987) 101-108.

23. A.M. Pooler, D.H. Guez, R. Benedictus, R.J. Wurtman, Uridine enhances neu- rite outgrowth in nerve growth factor-differentiated pheochromocytoma cells, Neuroscience 134 (2005) 207-214.

24. U.I. Richardson, C.J. Watkins, C. Pierre, I.H. Ulus, R.J. Wurtman, Stimulation of CDP-choline synthesis by uridine or cytidine in PC12 rat pheochromocytoma cells, Brain Res. 971 (2003) 161-167.

25. T. Sakamoto, M. Cansev, R.J. Wurtman, Oral supplementation with docosahe- xaenoic acid and uridine-51 -monophosphate increases dendritic spine density in adult gerbil hippocampus, Brain Res. 1182 (2007) 5059.

26. M.A. Tagin, C.G. Woolcott, M.J. Vincer, R.K. Whyte, D.A. Stinson, Hypother- mia for neonatal hypoxic ischemic encephalopathy: an updated system- atic review and meta-analysis, Arch. Pediatr. Adolesc. Med. 166 (2012) 558-566.

27. L. Thorell, L-B. Sjoberg, O. Hernell, Nucleotides in human milk: sources and metabolism by the newborn infant, Pediatric Res. 40 (1996) 845-852.

28. I.H. Ulus, C.J. Watkins, M. Cansev, R.J. Wurtman, Cytidine and uridine increase striatal CDP-choline levels without decreasing acetylcholine synthesis or release, Cell. Mol. Neurobiol. 26 (2006) 563-577.

29. C.J. van Groeningen, G.J. Peters, J.C. Nadal, E. Laurensse, H.M. Pinedo, Clinical and pharmacologic study of orally administered uridine, J. Natl. Cancer Inst. 83 (1991) 437-441.

30. S.J. Vanucci, H. Hagberg, Hypoxia-ischemia in the immature brain, J. Exp. Biol. 207 (2004) 3149-3154.

31. R.C. Vannucci, S.J. Vanucci, A model of perinatal hypoxic-ischemic brain dam- age, Ann. N.Y. Acad. Sci. 835 (1997) 234-429.

32. R.C. Vannucci, S.J. Vanucci, Perinatal hypoxic-ischemic brain damage: evolu- tion of an animal model, Dev. Neurosci. 27 (2005) 81-86.

33. J.J. Volpe, Hypoxic-ischemic encephalopathy, in: J.J. Volpe (Ed.), Neurology of the Newborn, WB Saunders, Philadelphia, PA, 2001, pp. 245-480.

34. P. Wintermark, Current controversies in newer therapies to treat birth asphyxia, Int. J. Pediatr. 2011 (2011) 848413.

35. R.J. Wurtman, M. Cansev, T. Sakamoto, I.H. Ulus, Use of phosphatide precursors to promote synaptogenesis, Annu. Rev. Nutr. 29 (2009) 59-87.

36. R.J. Wurtman, M. Regan, I.H. Ulus, L. Yu, Effect of oral CDP-choline on plasma choline and uridine levels in humans, Biochem. Pharmacol. 60 (2000) 989-992.

37. T. Xiong, Y. Qu, D. Mu, D. Ferriero, Erythropoietin for neonatal brain injury: opportunity and challenge, Int. J. Dev. Neurosci. 29 (2011) 583-591. 



\title{
Chapter
}

\author{
Possible neuroprotective effects of \\ magnesium sulphate and melatonin as both \\ pre- and post-treatment in a neonatal \\ hypoxic-ischemic rat model
}

Published as: Cetinkaya M, Alkan T, Ozyener F, Kafa IM, Kurt MA, Koksal N. Possible neuroprotective effects of magnesium sulphate and melatonin as both pre- and posttreatment in a neonatal hypoxic-ischemic rat model. Neonatology 2011; 99: 302-310 


\section{Abstract}

\section{Background:}

Perinatal hypoxia-ischemia is a major cause of mortality and long-term neurological deficits.

\section{Objectives:}

The objective of this study was to compare the effects of two neuroprotective agents; magnesium sulfate and melatonin, administered alone or in combination, on brain infarct volume and TUNEL positivity in a neonatal hypoxic-ischemic (HI) rat model.

\section{Methods:}

After being anesthetized, 7-day-old pups ( $n=80)$ underwent ischemia followed by exposure to hypoxia for $2 \mathrm{~h}$. The pups were then divided equally and randomly into 4 groups in order to receive the vehicle (saline, control group), magnesium sulfate, melatonin or a combination of magnesium sulfate and melatonin. Treatments were administered intraperitoneally three times; the first being just before ischemia, the second after hypoxia and the third $24 \mathrm{~h}$ after the second dose. The pups were sacrificed on postnatal day 10 , their brains harvested and evaluated for infarct volume and neuronal apoptosis.

\section{Results:}

Percent infarcted brain volume was significantly reduced in pups receiving the drugs (either magnesium sulfate, melatonin or their combination) compared with those receiving the vehicle. In addition, TUNEL staining showed markedly reduced numbers of TUNEL-positive cells per unit area in the CA1, CA3 and dentate gyrus regions of the hippocampus and in the cortex. However, no statistically significant differences were found regarding percent infarcted brain volume and number of TUNEL-positive cells among the drug-treated groups.

\section{Conclusions:}

Magnesium sulfate and melatonin, two agents acting at different stages of $\mathrm{HI}$ brain damage, administered either alone or in combination, significantly reduced the percent infarcted brain volume and TUNEL positivity, suggesting that these agents may confer a possible benefit in the treatment of infants with $\mathrm{HI}$ encephalopathy. 


\section{Introduction}

Perinatal hypoxic-ischemic ( $\mathrm{HI}$ ) brain damage is a major cause of neonatal mortality and severe long-term neurological deficits $[1,2]$. HI insult is an evolving process that is initiated during the insult and extends into the recovery period which is amenable to neuroprotective interventions [3, 4].

Magnesium sulfate $\left(\mathrm{MgSO}_{4}\right)$ is an NMDA receptor antagonist which prevents excitotoxic calcium-induced injury through the noncompetitive voltage-dependent inhibition of NMDA receptor, which reduces calcium entry into the cell [5]. Although conflicting data exist on the beneficial effects of magnesium in experimental and clinical neonatal HI models [6-17], magnesium bears major advantages as a neuroprotective agent in that it can be safely administered to newborn infants with severe perinatal asphyxia $[8,9]$.

Melatonin is an endogenously produced indoleamine that is primarily formed by the pineal gland. It is a potent free radical scavenger which also acts as an indirect antioxidant $[18,19]$. Melatonin has the ability of crossing all morphophysiological barriers and therefore is distributed widely in tissues, cells and subcellular compartments including the brain. Various studies reported that melatonin might act as a neuroprotective agent in neonatal hypoxia-ischemia [20-22] and, like $\mathrm{MgSO}_{4}$, melatonin has also been used clinically in neonates [23-25].

The aim of this study was to evaluate the effects of two neuroprotective agents, $\mathrm{MgSO}_{4}$ and melatonin, administered alone or in combination, on brain infarct area and TUNEL positivity with the expectation that these two agents might prevent apoptosis and provide possible neuroprotection in a neonatal $\mathrm{HI}$ rat model.

\section{Materials and Methods}

\section{Animals}

All experimental protocols and procedures were reviewed by the Animal Care and Use Committee of the Uludag University, Faculty of Medicine, and all were in accordance with the guidelines provided by the Experimental Animal Laboratory. A modification of LevineRice preparation was used as a model for perinatal $\mathrm{HI}$ brain injury as previously reported [26]. Dated pregnant Sprague-Dawley rats were housed in individual cages under standard conditions with free access to water and laboratory chow. The day of spontaneous vaginal delivery was considered postnatal day 1 (PND1) for the pups and a total of 80 offspring were reared with their dams until initiation of the experiments on day 7 (PND7), which coincides with 32- to 34-week-old preterm human brains [27]. Rat pups of either sex weighing 12-16 g were used in the experiments. 


\section{Study Design}

PND7 rats $(n=80)$ subjected to $\mathrm{HI}$ were divided equally and randomly into 4 groups and injected intraperitoneally with the following: vehicle (saline, $0.1 \mathrm{ml}$ ), magnesium sulfate $\left(\mathrm{MgSO}_{4}, 275 \mathrm{mg} / \mathrm{kg}\right)$, melatonin (Mel, $20 \mathrm{mg} / \mathrm{kg}$ ) and a combination of magnesium sulfate and melatonin $\left(\mathrm{MgSO}_{4}[275 \mathrm{mg} / \mathrm{kg}]+\mathrm{Mel}[20 \mathrm{mg} / \mathrm{kg}]\right)$. Treatments were given three times; the first being just before ischemia, the second after hypoxia and the third $24 \mathrm{~h}$ after the second dose. Given the ability that they are able to cross the placenta $[28,29]$, these agents were chosen in that they might provide neuroprotection at the initial stage of hypoxiaischemia. A schematic diagram of the experimental protocol is shown in figure 1.

The dose of magnesium sulfate used in our study $(275 \mathrm{mg} / \mathrm{kg})$ was slightly higher than those used in previous experimental and clinical models of perinatal HI brain injury [7, 8, 30]. Moreover, the dose of melatonin used in our study $(20 \mathrm{mg} / \mathrm{kg})$ was also within the upper range of melatonin doses which were reported to exhibit the greatest neuroprotection in previous studies (range 5-20 mg/kg) [20-22].

Melatonin (Sigma, St. Louis, Mo., USA) was dissolved freshly in absolute ethanol and liquidized in isotonic sodium chloride $(0.9 \% \mathrm{NaCl})$ to obtain a final concentration of 1:10 solution.

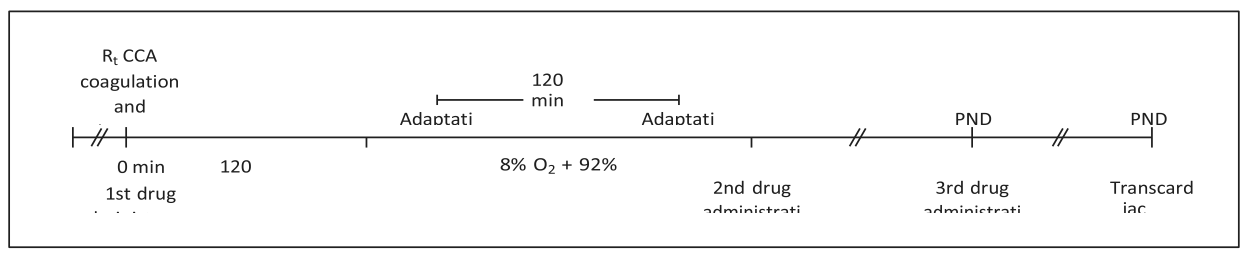

Fig. 1. Experimental steps.

\section{Induction of HI Brain Injury and Treatment Groups}

Pups were anesthetized lightly with inhaled isoflurane, the right carotid artery was identified through a longitudinal neck incision, isolated from the nerve and vein, and permanent surgical ligation with 5.0 surgical silk plus coagulation of carotid artery was performed. The procedure was performed at room temperature (RT) and total time of surgery never exceeded $3 \mathrm{~min}$. Animals exhibiting bleeding during ligation or respiratory arrest due to anesthesia were excluded from the study. After closure of the neck incision, pups were allowed to recover for $15 \mathrm{~min}$ and then returned to the nest temperature. Following a 2-hour period of nesting, the pups were placed into chambers partially submerged in a $37^{\circ} \mathrm{C}$ water bath to maintain a constant thermal environment and exposed to hypoxia with a warm humidified 8/92\% oxygen/ nitrogen mixture for $2 \mathrm{~h}$. Upon retrieval from the hypoxia chambers, the pups were allowed to recover for $72 \mathrm{~h}$ with their dams. Following deep isoflurane anesthesia, cardiac perfusion was performed with phosphate buffer solution containing 4\% paraformaldehyde and brains were removed for evaluation of hypoxic damage and immunohistochemical analysis. 


\section{Measurement of Infarcted Volume}

Cardiac perfusion was performed $72 \mathrm{~h}$ after hypoxia (PND10). Pups were sacrificed immediately ( $n=6$, each group), and the brains were quickly removed, placed in ice-cold saline for $5 \mathrm{~min}$, and $2 \mathrm{~mm}$-thick coronal sections were obtained starting from the frontal pole using a neonatal brain matrix (Ted Pella, Calif., USA). The slices were incubated in $2 \%$ 2,3,5-triphenyl-tetrazolium chloride (TTC, dissolved in saline) for $15 \mathrm{~min}$ at $37^{\circ} \mathrm{C}$, fixed by immersion in $10 \%$ formalin, and images acquired with a Sony Cybershot DSC-F717 digital camera attached to a Nikon 4S-2 Alphaphot light microscope. The white areas of infarct were quantified using image-analysis software (Scion Corporation version 4.02; $\mathrm{NIH}$, Bethesda, Md., USA). The mean infarct volume per slice was obtained from the product of the average thickness of a slice $(2 \mathrm{~mm})$ and the area of infarct in that section. The percent infarcted volume was calculated with an indirect method described by Swanson et al. [31].

\section{Immunohistochemistry}

Rats anesthetized by inhaled isoflurane were perfused with $4 \%$ paraformaldehyde in 0.1 $M$ phosphate-buffered saline (PBS) $(\mathrm{pH}$ 7.4). Brains were then removed, postfixed overnight, embedded in paraffin and $5-\mu \mathrm{m}$ coronal sections were obtained using a microtome. Sections were chosen according to a systematic random sampling procedure, and then mounted onto poly-L-lysine-coated slides (Histobond adhesion slides, Marienfeld, Germany). An in situ cell death detection POD kit (Roche Molecular Biochemicals, 11684817910, Germany) was used for TUNEL technique (terminal deoxynucleotidyl transferase (TdT)-mediated deoxyuridine triphosphate (dUTP)-biotin nick end-labeling) and the sections stained according to the manufacturer's protocol for paraffin-embedded tissues. Briefly, sections were heated at $60^{\circ} \mathrm{C}$ for $45 \mathrm{~min}$ followed by washing in xylene and rehydration through graded series of alcohol (ethanol 100, 95, 80, 70 and 40\%) and washed in distilled water. They were then incubated in $20 \mu \mathrm{g} / \mathrm{ml}$ proteinase K solution (Roche Applied Sciences, 031158444001, Germany) for 20 min at $\mathrm{RT}$ and immersed in $3 \% \mathrm{H}_{2} \mathrm{O}_{2}$ in methanol for 10 min. Permeabilization with Triton $\mathrm{X}$ (Sigma-Aldrich, T-9284, USA) for 10 min was performed on ice. The sections were then incubated with $35 \mu \mathrm{l}$ TUNEL reaction mixture at $37^{\circ} \mathrm{C}$ in a humidified chamber for $1 \mathrm{~h}$ followed by converter-POD treatment at $37^{\circ} \mathrm{C}$ for $30 \mathrm{~min}$. Diaminobenzidine tetrahydrochloride (DAB) (50 $\mu$ 1:9, diaminobenzidine/ metal concentrate: peroxidase buffer, Roche Applied Sciences, 11718096001, Germany) was used as the chromogene and the slides were counterstained with hematoxylin (Harris's hematoxylin, SigmaAldrich, HHS16, USA), and coverslipped (DPX mountant, Fluka, 44581, USA). $35 \mu$ I DNase $(200 \mu \mathrm{g} / \mathrm{ml}$ DNase I (Roche Applied Sciences, Germany) in DNase buffer (40 mM Tris HCl, $\mathrm{pH} 7.610 \mathrm{mM} \mathrm{NaCl}, 6 \mathrm{mM} \mathrm{MgCl} 2,10 \mathrm{mM} \mathrm{CaCl}_{2}$ ) was used for positive controls for $10 \mathrm{~min}$ at $37^{\circ} \mathrm{C}$, while alternate sections were incubated in the absence of a TUNEL reaction mixture for negative controls. TUNEL-positive cells with characteristic nuclear 
morphological changes including chromatin condensation, hypersegmentation of nuclear chromatin and shrunken (pyknotic) appearance were counted using an ocular micrometer grid randomly on the CA1, CA3 and dentate gyrus (DG) areas of the hippocampus.

Immunohistochemistry staining for caspase-3 was performed using the avidin biotin complex $(A B C)$ technique. Sections were heated at $60^{\circ} \mathrm{C}$ for $1 \mathrm{~h}$ followed by washing in xylene and rehydration through graded series of alcohol (ethanol 100, 95, 80, 70 and $40 \%$ ) and washed in distilled water. They were then placed in $10 \mathrm{mM}$ citrate buffer at $\mathrm{pH}$ 6.0 and treated with $750 \mathrm{~W}$ in a microwave for $10 \mathrm{~min}$ for antigen retrieval and cooled for 20 min at RT. Slides were then immersed in $3 \% \mathrm{H}_{2} \mathrm{O}_{2}$ for 10 min and incubated in $10 \%$ goat serum in Tris buffer (0.01 M Tris $\mathrm{HCl}, \mathrm{pH} 7.4,1 \mathrm{mM} \mathrm{CaCl}, 1 \mathrm{mM} \mathrm{MgCl} 2,0.1 \%$ Triton X-100) for $30 \mathrm{~min}$ at RT. After blocking, slides were stained with caspase-3 (1:100; Chemicon, AB3623-rabbit, USA) antibody which is diluted in same blocking buffer, overnight at $4^{\circ} \mathrm{C}$. Secondary antibody (biotinylated anti-rabbit IgG, Vectastain Elite $A B C$ Kit, USA) was applied for $1 \mathrm{~h}$ at RT. Sections were then treated with $100 \mu \mathrm{l}$ of $A B C$ (Vectastain Elite $A B C$ Kit) and finally visualized using DAB $(50 \mu$ 1:9, diaminobenzidine/metal concentrate : peroxidase buffer) and counter- stained with hematoxylin.

\section{Cell Count}

A total of at least 200 cells counted in 7 fields were chosen randomly at $x 400$ magnification for evaluation of TUNEL-positive cells' index with respect to their morphological characteristics. In addition, all positive cells for caspase-3 immunoreactivity were counted at $\times 200$ magnification.

\section{Statistics}

Data regarding the 'percent infarcted volume' obtained by TTC staining were analyzed using 'Kruskal-Wallis one-way ANOVA' between groups. Further analysis between groups were made using the 'Mann-Whitney $U$ test' if significant differences were found by ANOVA. TUNEL-positive cell counts were also analyzed using 'Kruskal-Wallis one-way ANOVA' and 'Mann-Whitney U test'. Sigma-stat 3.0 and SPPS 16.0 programs were used for the statistical tests and significance assumed at $95 \%$ confidence $(p<0.05)$.

\section{Results}

Percent infarcted volume was significantly reduced following melatonin (18.39 \pm 1.31 ), $\mathrm{MgSO}_{4}(18.81 \pm 2.25)$ or melatonin $+\mathrm{MgSO}_{4}(14.66 \pm 2.97)$ administration compared with that in control ischemic animals (42.45 \pm 4.63$)$. There was no statistically significant difference in terms of percent infarcted volume between the melatonin, $\mathrm{MgSO}_{4}$ and melatonin $+\mathrm{MgSO}_{4}$ treated groups ( $\left.p>0.05\right)$ (fig. 2, 3) 
TUNEL stain revealed markedly reduced numbers of TUNEL-positive cells in the CA1, CA3 and DG regions of the hippocampus in pups receiving the drugs $(p<0.001)$. A similar reduction in TUNEL-positive cell count per unit area was also observed in the cortex of the melatonin $(2.19 \pm 0.13), \mathrm{MgSO}_{4}(4.91 \pm 0.44)$ and melatonin $+\mathrm{MgSO}_{4}(2.15 \pm 0.25)$ treated groups compared with that in control ischemic animals (33.78 \pm 1.75 ) (fig. 4-6). However, no statistically significant difference was found for TUNEL-positive cell count between the melatonin, $\mathrm{MgSO}_{4}$ and melatonin $+\mathrm{MgSO}_{4}$ treated groups ( $\left.\mathrm{p}>0.05\right)$. In addition, the number of caspase-3-positive cells was significantly lower in the drugtreated groups compared with the control group (fig. 7, 8); again no significant difference was found among treated groups in terms of caspase- 3 immunoreactivity.

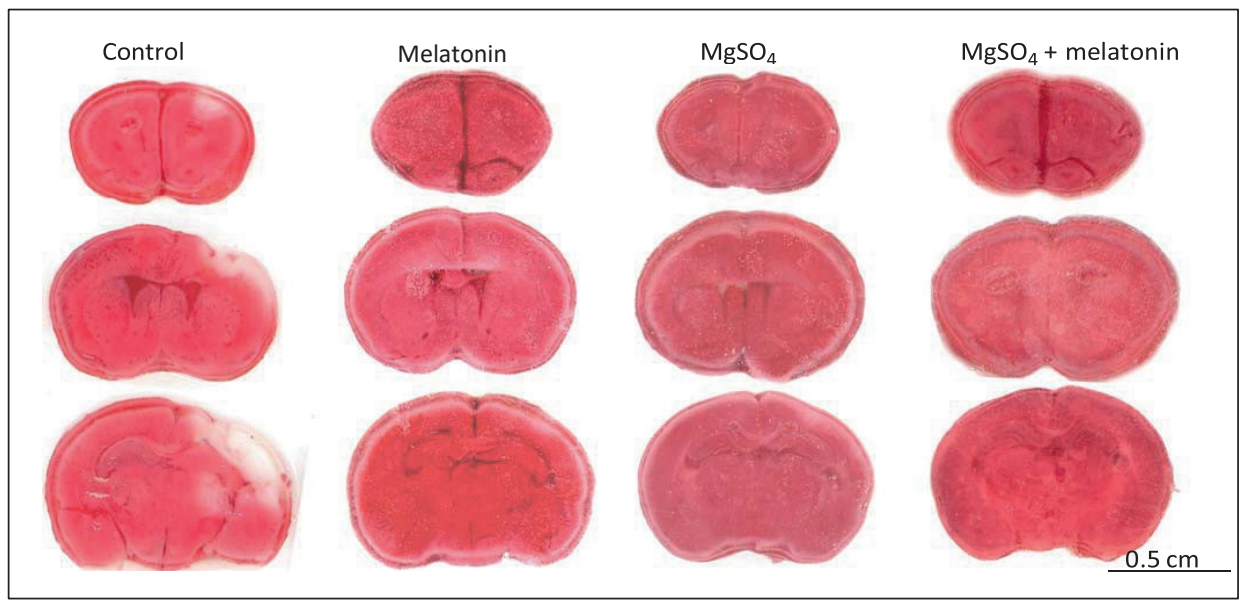

Fig. 2. TTC-stained coronal sections from each experimental group.

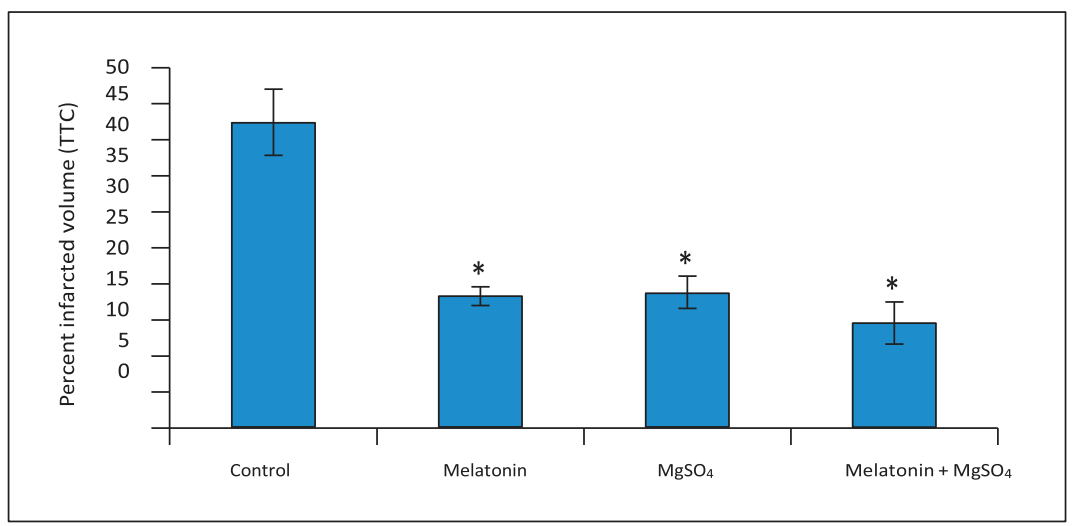

Fig. 3. Percent infarcted volumes from brains of rat pups in each experimental group. ${ }^{*} p \sim 0.05$. 


\section{Chapter 7}

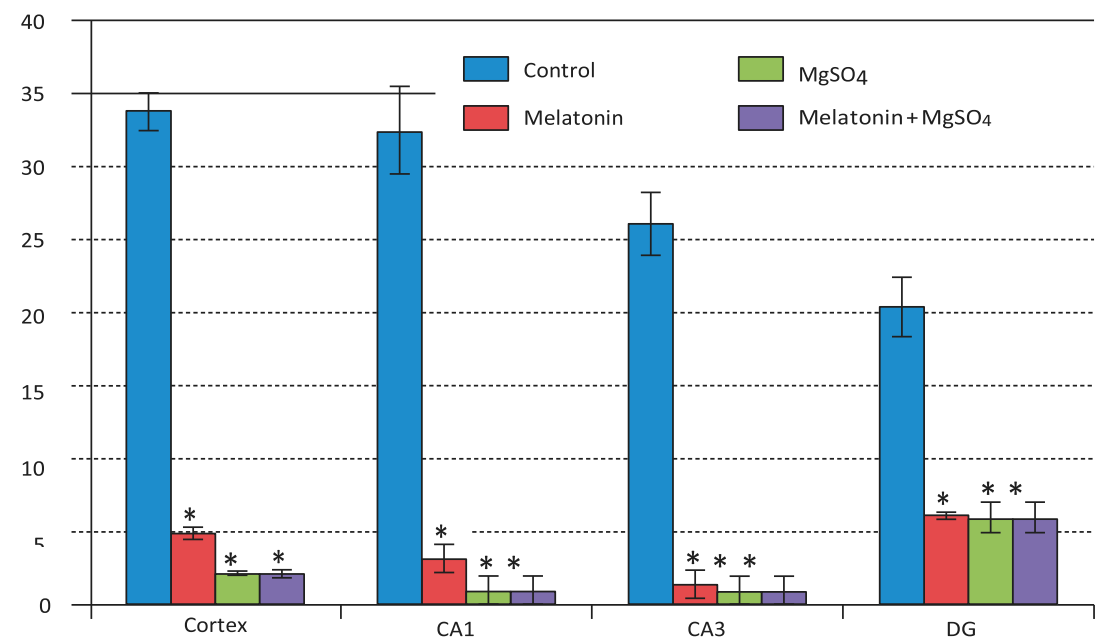

Fig. 4. TUNEL-positive cell count per unit area of cortex and hippocampus in each experimental group. ${ }^{*} p \sim$ 0.05 . 


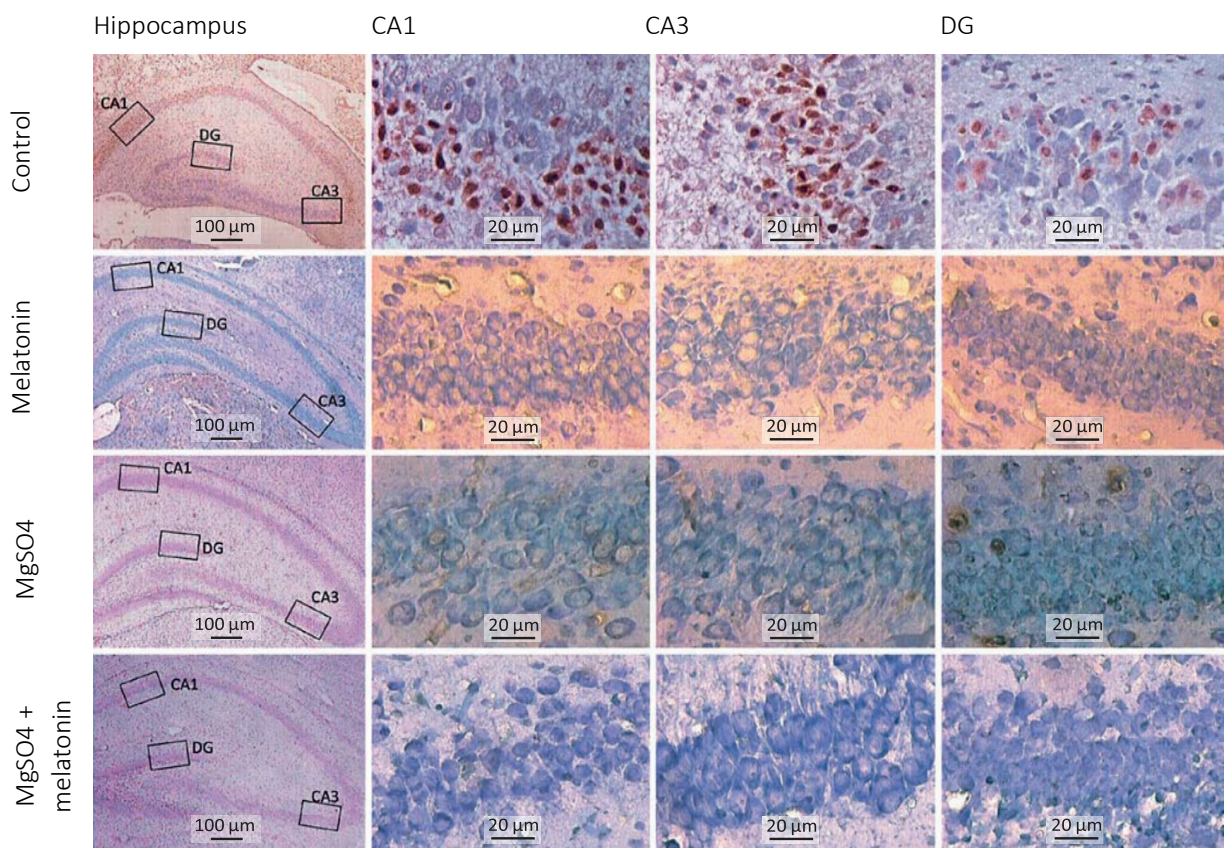

Fig. 5. TUNEL staining in the hippocampus.
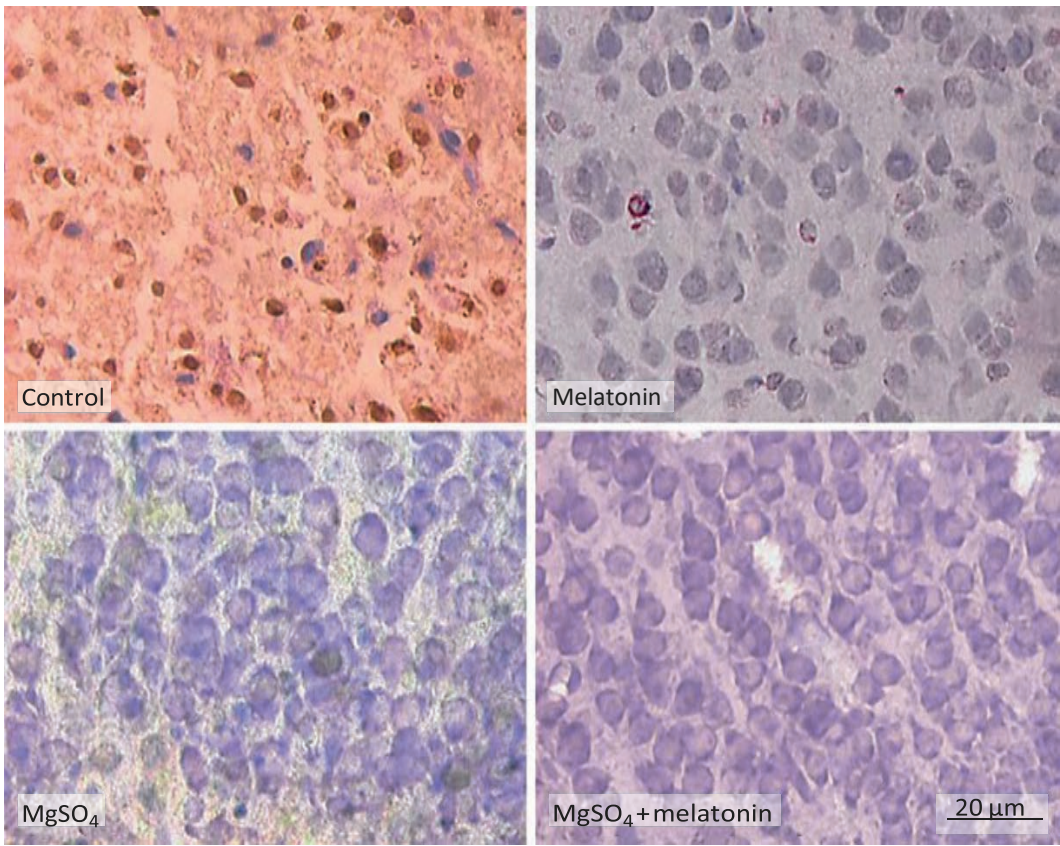

Fig. 6. TUNEL staining in the cortex.

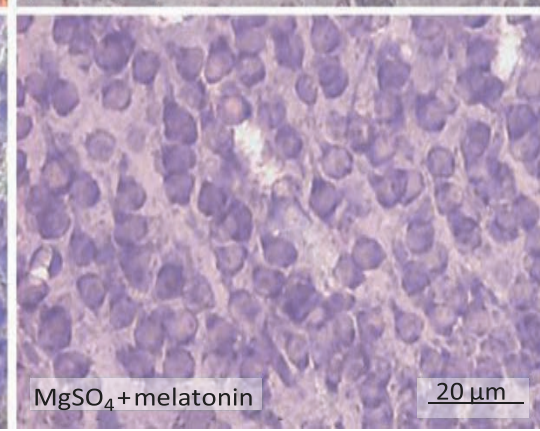




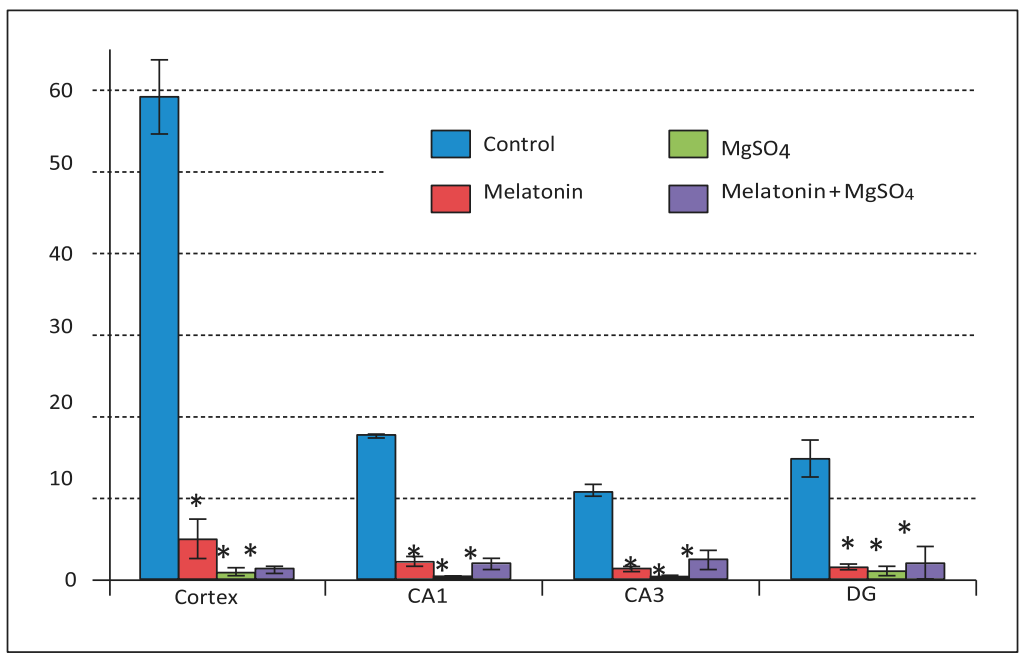

Fig. 7. Caspase-3-positive cell count per unit area of cortex and hippocampus in each experimental group. * $p$ 0.05 .

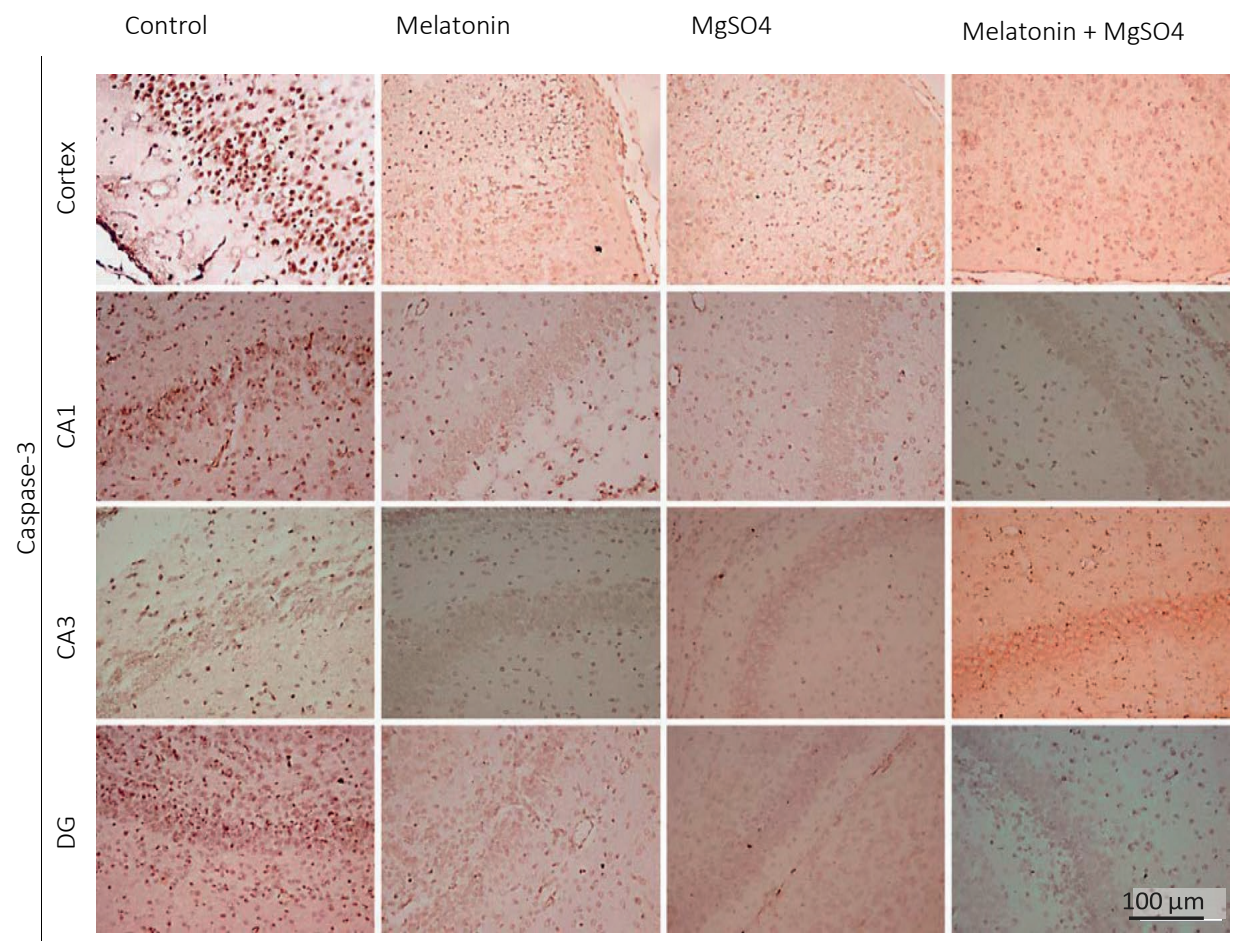

Fig.8. Caspase-3 immunoreactivity in cortex and hippocampal areas of each group. 


\section{Discussion}

These data show that $\mathrm{MgSO}_{4}$ and melatonin, agents acting on different levels of the hypoxia-ischemia cascade, exhibit significant neuroprotection in a neonatal $\mathrm{HI}$ rat model by reducing the TUNEL-positive cell count and brain injury when administered either alone or in combination. However, there was no additive benefit from combination treatment beyond that observed after individual administration of either $\mathrm{MgSO}_{4}$ or melatonin.

The studies that investigated the potential neuroprotective effect of magnesium have revealed conflicting data [3, 6-17]. $\mathrm{MgSO}_{4}$ given 1, 12 and $24 \mathrm{~h}$ after $\mathrm{HI}$ insult did not decrease the severity of delayed cerebral energy failure [12]. Similarly, $\mathrm{MgSO}_{4}$ treatment did not induce neuroprotection in several brain regions including hippocampus, cerebellum, cerebral cortex, caudate nucleus, thalamus and striatum [13]. In a study on lambs, repeated doses of $\mathrm{MgSO}_{4}$ given before and during hypoxia did not provide significant neuroprotection [16]. In contrast, discernible neuroprotective effects of $\mathrm{MgSO}_{4}$ have been demonstrated in other neonatal studies [7, 30, 32]. Turkyilmaz et al. [7] reported that $\mathrm{MgSO}_{4}(200 \mathrm{mg} / \mathrm{kg} /$ dose) administered before the $\mathrm{HI}$ insult reduced apoptosis in the hippocampus in a neonatal $\mathrm{HI}$ rat model, while a similar dose given after $\mathrm{HI}$ insult did not show any significant neuroprotective effect. Post-asphyxial $\mathrm{MgSO}_{4}$ treatment resulted in significant decreases in both the incidence of cyst formation and the severity of neuronal loss in cerebral cortex and hippocampus [30]. Recently, $\mathrm{MgSO}_{4}$ administration after $\mathrm{HI}$ insult was reported to limit the neuronal damage and also prevented the sensorimotor deficits and brain injury induced by hypoxia- ischemia [32]. In our study, $\mathrm{MgSO}_{4}$ given just before ischemia and after hypoxia significantly decreased brain infarct volume and TUNEL-positive cell count in a rat model of neonatal HI brain damage employing rat pups of 7 days of age (PND7) which coincides with 32- to 34-weekold preterm human brain [27]. The conflicting results on the effects of magnesium in experimental neonatal $\mathrm{HI}$ brain injury models may be associated with administration time and dose of the drug or, alternatively, with the design of studies.

The neuroprotective effect of magnesium was also evaluated clinically in infants with $\mathrm{HI}$ encephalopathy (HIE). Groenendaal et al. [11] first reported that $\mathrm{MgSO}_{4}$ did not have a positive effect on the electroencephalography patterns of asphyxiated term infants. However, postnatal $\mathrm{MgSO}_{4}$ infusion ( $250 \mathrm{mg} / \mathrm{kg} /$ dose for $6 \mathrm{~h}$ ) for 3 days after hypoxia improved short-term outcomes of infants with severe birth asphyxia [8]. In addition, postnatal magnesium treatment decreased mortality and morbidity as well as it improved the neurodevelopmental out- come of infants with severe asphyxia [33]. Recently, 3 doses of $\mathrm{MgSO}_{4}$ infusion after perinatal hypoxia resulted in improved neurological outcomes of infants with severe perinatal asphyxia [9]. The treatment regimen employed in the present study, i.e. administering three consecutive doses the first of which was given just before the $\mathrm{HI}$ insult, was derived from the above-mentioned previous studies $[8,9,33]$. 
$\mathrm{MgSO}_{4}$ may induce systemic hypotension, especially at high doses [33, 34]. Levene et al. [34] reported that high dose $(400 \mathrm{mg} / \mathrm{kg}) \mathrm{MgSO}_{4}$ administration was associated with an increased risk of hypotension, whereas a lower dose $(250 \mathrm{mg} / \mathrm{kg})$ did not induce hypotension except in respiratory depression. Ichiba et al. [33] administered $\mathrm{MgSO}_{4}$ infusion $(250 \mathrm{mg} / \mathrm{kg}$ ) in combination with dopamine infusion and reported no hypotension in infants with severe birth asphyxia. Therefore, caution is required in the administration of high doses of $\mathrm{MgSO}_{4}$ to avoid systemic hypotension.

Melatonin was reported to be beneficial in neonatal HI brain damage [20]. Recently, melatonin, administered before and after hypoxia, was reported to exhibit a significant neuroprotective effect and also decrease behavioral problems and learning deficits at long-term follow-up [22]. In the present study, administration of melatonin resulted in a significant neuroprotective effect in a rat model of $\mathrm{HI}$ brain damage. A major advantage of melatonin is its wide and safe usage in neonates in clinical situations such as sepsis, bronchopulmonary dysplasia and asphyxia [23-25].

To our knowledge, the neuroprotective effect of melatonin as a part of combination therapy in a neonatal $\mathrm{HI}$ model has not yet been investigated. Therefore, there are no data about the effectiveness of melatonin as a combination therapy in hypoxia-ischemia. The results of the present study showed a significant neuroprotective effect of melatonin and $\mathrm{MgSO}_{4}$ combination therapy. In a transient global ischemia model, $\mathrm{MgSO}_{4}$ had neuroprotective effect only when it was combined with mild hypothermia [35]. It was also reported that post-ischemic combination treatment with 24-hour hypothermia and magnesium infusion was more effective in reducing CA1 neuronal death than either treatment used alone [36]. Consistently, that combined neuroprotective therapies might be necessary for increasing the therapeutic time window for protection and repair in neonatal $\mathrm{HI}$ brain damage studies was recently suggested [37]. In addition, in a recent review, Fatemi et al. [38] suggested that the best outcome might be achieved with a multimodal therapeutic approach such as a combination of glutamate receptor antagonists with antioxidants and hypothermia. Consistent with these suggestions, in the present study, combination of $\mathrm{MgSO}_{4}$ as an NMDA antagonist with melatonin exhibited a significant neuroprotective effect. However, this benefit was not superior to that observed after single therapies. Hagberg et al. [39] proposed that all stages of the HI cascade might cause mitochondrial stress which subsequently results in mitochondrial outer membrane permeabilization that leads to irreversible caspase- and non-caspasedependent cell death. This raises the possibility that $\mathrm{MgSO}_{4}$ and melatonin might have worked through a common pathway and, hence, did not provide any complimentary effect in our experimental setting. Therefore, future studies are warranted to evaluate the effectiveness of multimodal treatment models in neonatal HI brain damage.

The experimental procedure in our study was designed in a way that it mimicked the clinical presentation of HIE and the treatments were given three times (the first being just before ischemia, the second after hypoxia and the third $24 \mathrm{~h}$ after the second dose). Inasmuch as the treatments were first given just before ischemia, data obtained in our 
prophylaxis study might reflect a possible neuroprotective effect of $\mathrm{MgSO}_{4}$ and melatonin. Since these agents are capable of crossing the placenta $[28,29]$, they might confer benefit in the treatment of hypoxia- ischemia in the case that HIE is recognized in utero. Consistent with this suggestion, previous studies reported beneficial effects of either melatonin or $\mathrm{MgSO}_{4}$ in $\mathrm{HIE}$ when given prophylactically to mothers. In a recent study in a spiny mouse model, melatonin administered to mothers in late pregnancy as a prophylaxis treatment provided neuroprotection in pups subjected to birth asphyxia [29]. Similarly, maternal administration of melatonin within $1 \mathrm{~h}$ after an ischemic/oxidative episode was found to be preventive against cerebral damage in neonatal rats [40]. Zylinska et al. [28] reported that antenatal $\mathrm{MgSO}_{4}$ treatment might affect calcium homeostasis and antioxidant reserve in the erythrocytes of preterm infants. This might reflect the possible neuroprotective effect of agents crossing the placenta such as melatonin and $\mathrm{MgSO}_{4}$ in neonatal $\mathrm{HI}$ brain damage, suggesting that these agents could be administered to dams prophylactically if in utero hypoxia is suspected.

However, the limitation of the present study is that our experimental procedure did not allow us to test whether administration of $\mathrm{MgSO}_{4}$ and melatonin following $\mathrm{HI}$ is preventive. Therefore, we are unable to suggest that treatment with these agents may also confer benefit in treatment of HIE in the case that $\mathrm{HI}$ is not determined in utero.

Magnesium was shown to increase cerebral blood flow in humans and animals and was suggested to cause dilation of smaller arteries or collateral and/or anastomotic blood flow pathways [41]. Acute melatonin administration was suggested to diminish the hypoperfusion-induced cerebral ischemia by increasing the cerebrovascular dilatory capacity in rats [42]. Therefore, $\mathrm{MgSO}_{4}$ and/or melatonin might have exhibited a possible neuroprotective effect by improving cerebrovascular blood flow and anastomotic perfusion.

In conclusion, both $\mathrm{MgSO}_{4}$ and melatonin, two agents acting at different stages of $\mathrm{HI}$, used either alone or in combination, significantly decreased the percent infarcted area and TUNEL positivity in a neonatal $\mathrm{HI}$ rat model. However, the combination therapy did not show any superior neuroprotective effect compared with that observed after individual administration of either $\mathrm{MgSO}_{4}$ or melatonin. Although it would be premature to draw any strict parallels between the neuroprotective effect of combined $\mathrm{MgSO}_{4}$ and melatonin therapy in a rat model of $\mathrm{HI}$ brain injury and clinical treatment of hypoxiaischemia in infants, the data provided here render a valuable reference point for future clinical investigation and these two agents may be used in combination in the clinical treatment of infants with HIE.

\section{Acknowledgements}

This study was supported by TUBITAK (Turkish Scientific and Technical Research Council), project number 108S128. We also thank Dr. Mehmet Cansev for his valuable suggestions during revision of the manuscript. 


\section{References}

1. Vanucci SJ, Hagberg H: Hypoxia-ischemia in the immature brain. J Exp Biol 2004;207: 3149-3154.

2. Volpe JJ: Hypoxic-ischemic encephalopathy; in Volpe JJ (ed): Neurology of the Newborn. Philadelphia, Saunders, 2001.

3. Shalak L, Perlman JM: Hypoxic-ischemic brain injury in the term infant-current concepts. Early Hum Dev 2004;80:125-141.

4. van Bel F, Groenendaal F: Long-term pharmacologic neuroprotection after birth asphyxia: where do we stand? Neonatology 2008;94:203-210.

5. Ovbiagele B, Kidwell CS, Starkman S, Saver JL: Neuroprotective agents for the treatment of acute ischemic stroke. Curr Neurol Neurosci Rep 2003;3:9-20.

6. Hoffman DJ, Marro PJ, McGowan JE, Mishra OP, Delivoria-Papadopoulos M: Protective effect of MgSO4 infusion on NMDA receptor binding characteristics during cere- bral cortical hypoxia in the newborn piglet. Brain Res 1994;644:144-149.

7. Turkyilmaz C, Turkyilmaz Z, Atalay Y, Soy- lemezoglu F, Celasun B: Magnesium pre- treatment reduces neuronal apoptosis in newborn rats in hypoxia-ischemia. Brain Res 2002;955:133-137.

8. Ichiba H, Tamai H, Negishi H, Ueda T, Kim TJ, Sumida Y, Takahashi Y, Fujinaga H, Minami H, Kansai Magnesium Study Group: Randomized controlled trial of magnesium sulfate infusion for severe birth asphyxia. Pediatr Int 2002;44:505-509.

9. Bhat MA, Charoo BA, Bhat JI, Ahmad SM, Ali SW: Magnesium sulfate in severe perina- tal asphyxia: a randomized, placebo-con- trolled trial. Pediatrics 2009;123:e764-e769.

10. McDonald JW, Silverstein FS, Johnston MV: Magnesium reduces N-methyl-D-aspartate (NMDA)-mediated brain injury in perinatal rats. Neurosci Lett 1994;644:144-149.

11. Groenendaal F, Rademaker CMA, Toet MC, de Vries LS: Effects of magnesium sulfate on amplitudeintegrated continuous EEG in as- phyxiated term neonates. Acta Paediatr 2002;91:1073-1077.

12. Penrice J, Amess PN, Punwani S, Punwani S, Wylezinska M, Tyszczuk L, D'Souza P, Ed- wards AD, Cady EB, Wyatt JS, Reynolds EO: Magnesium sulfate after transient hypoxia- ischemia fails to prevent delayed cerebral en- ergy failure in the newborn piglet. Pediatr Res 1997;41:443-447.

13. Greenwood K, Cox P, Mehmet H, Penrice J, Amess PN, Cady EB, Wyatt JS, Edwards AD: Magnesium sulfate after transient hypoxia- ischemia in the newborn piglet does not pro- tect against cerebral damage. Pediatr Res 2000;48:346-350.

14. Spandou E, Soubasi V, Papoutsopoulou S, Augoustides-Savvopoulou P, Loizidis T, Pazaiti A, Karkavelas G, Guiba-Tziampiri O: Neuroprotective effect of long term MgSO4 administration after cerebral hypoxia-ischemia in newborn rats is related to the sever- ity of brain damage. Reprod Sci 2007;14:667-677.

15. Sameshima H, Ota A, Ikenoue T: Pretreat- ment with magnesium sulfate protects against hypoxic-ischemic brain injury but postasphyxial treatment worsens brain damage in seven-day-old rats. Am J Obstet Gynecol 1999;180:725-730.

16. de Haan HH, Gunn AJ, Williams CE, Heymann MA, Gluckman PD: Magnesium sulfate therapy during asphyxia in near-term fetal lambs does not compromise the fetus but does not reduce cerebral injury. Am J Obstet Gynecol 1997;176:18-27.

17. Dribben WH, Creeley CE, Wang HH, Smith DJ, Farber NB, Olney JW: High dose magnesium sulfate exposure induces apoptotic cell death in the developing neonatal mouse brain. Neonatology 2009;96:23-32.

18. Tan DX, Manchester LC, Terron MP, Flores LJ, Reiter RJ: One molecule, many derivatives: a never-ending interaction of melato- nin with reactive oxygen and nitrogen spe- cies? J Pineal Res 2007;42:28-42.

19. Tomas-Zapico C, Coto-Montes A: A pro- posed mechanism to explain the stimulatory effect of melatonin on antioxidative en- zymes. J Pineal Res 2005;39:99-104.

20. Tutunculer F, Eskiocak S, Basaran UN, Ekuklu G, Ayvaz S, Vatansever U: The protective role of melatonin in experimental hy- poxic brain damage. Pediatr Int 2005;47: 434-439. 
21. Welin AK, Svedin P, Lapatto R, Sultan B, Hagberg H, Gressens P, Kjellmer I, Mallard C: Melatonin reduces inflammation and cell death in white matter in the mid-gestation fetal sheep following umbilical cord occlusion. Pediatr Res 2007;61:153-158.

22. Carloni S, Perrone S, Buonocore G, Longini M, Proietti F, Balduini W: Melatonin protects from the long-term consequences of a neonatal hypoxic-ischemic brain injury in rats. J Pineal Res 2008;44:157-164.

23. Gitto E, Karbownik M, Reiter RJ, Tan DX, Cuzzocrea S, Chiurazzi P, Cordaro S, Corona G, Trimarchi G, Barberi I: Effects of melato- nin treatment in septic newborns. Pediatr Res 2001;50:756-760.

24. Gitto E, Reiter RJ, Amodio A, Romeo C, Cuz- zocrea E, Sabatino G, Buonocore G, Cordaro V, Trimarchi G, Barberi I: Early indicators of chronic lung disease in preterm infants with respiratory distress syndrome and their in- hibition by melatonin. J Pineal Res 2004;36: 250-255.

25. Fulia F, Gitto E, Cuzzocrea S, Reiter RJ, Dugo L, Gitto P, Barberi S, Cordaro S, Barberi I: Increased levels of malondialdehyde and ni- trite/nitrate in the blood of asphyxiated new- borns: reduction by melatonin. J Pineal Res 2001;31:343-349.

26. Vannucci RC, Vanucci SJ: A model of perinatal hypoxic-ischemic brain damage. Ann NY Acad Sci 1997;835:234-429.

27. Vannucci RC, Vannucci SJ: Perinatal Hypox-ic-ischemic brain damage: evolution of an animal model. Dev Neuroscience 2005;27: 81-86.

28. Zylinska L, Gulczynska E, Kozaczuk A: Changes in erythrocyte glutathione and plasma membrane calcium pump in preterm newborns treated antenatally with MgSO4. Neonatology 2008;94:272-278.

29. Hutton LC, Abbass M, Dickinson H, Ireland Z, Walker DW: Neuroprotective effects of melatonin in a model of birth asphyxia in the spiny mouse (Acomys cahirinus). Dev Neurosci 2009;31:437-451.

30. Sameshima $\mathrm{H}$, Ikenoue $\mathrm{T}$ : Long term magne- sium sulfate treatment as protection against hypoxic-ischemic brain injury in seven-day old rats. Am J Obstet Gynecol 2001;184:185-190.

31. Swanson RA, Morton MT, Tsao-Wu G, Savalos RA, Davidson C, Sharp FR: A semi- automated method for measuring brain in- farct volume. J Cereb Blood Flow Met 1990; 10:290-293.

32. Pazaiti A, Soubasi V, Spandou E, Karkavelas G, Georgiou T, Karalis P, Guiba-Tziampiri O: Evaluation of longlasting sensorimotor consequences following neonatal hypoxic- ischemic brain injury in rats: the neuroprotective role of MgSO4. Neonatology 2009;95: 33-40.

33. Ichiba H, Ykoi T, Tamai H, Ueda T, Kim TJ, Yamano T: Neurodevelopmental outcome of infants with birth asphyxia treated with magnesium sulfate. Pediatr Int 2006;48:70- 75.

34. Levene M, Blennow M, Whitelaw A, Hank $\varnothing$ E, Fellman V, Hartley R: Acute effects of two different doses of magnesium sulphate in infants with birth asphyxia. Arch Dis Child Fe- tal Neonatal Med 1995;73:174-177.

35. Zhu H, Meloni BP, Moore SR, Majda BT, Knuckey NW: Intravenous administration of magnesium is only neuroprotective following transient global ischemia when present with post-ischemic mild hypothermia. Brain Res 2004;1014:53-60.

36. Zhu H, Meloni BP, Bojarski C, Knuckey MW, Knuckey NW: Post-ischemic modest hypothermia (35 degrees C) combined with intra- venous magnesium is more effective at re- ducing CA1 neuronal death than either treatment used alone following global cerebral ischemia in rats. Exp Neurol 2005;193: 361-368.

37. Gonzalez FD, Ferriero DM: Neuroprotection in the newborn infant. Clin Perinatol 2009; 36:859-880.

38. Fatemi A, Wilson MA, Johnston MV: Hypoxic-ischemic encephalopathy in the term infant. Clin Perinatol 2009;26:835-858.

39. Hagberg H, Mallard C, Rousset Cl, Wang X: Apoptotic mechanisms in the immature brain: Involvement of mitochondria. J Child Neurol 2009;24:1141-1146.

40. Hamada F, Watanabe K, Wakatsuki A, Nagai R, Shinohara K, Hayashi Y, Imamura R, Fu- kaya T: Therapeutic effects of maternal melatonin administration on ischemia/reperfusion-induced oxidative cerebral damage in neonatal rats. Neonatology 2010;98:33-40.

41. Kim CR, Oh W, Stonestreet BS: Magnesium is a cerebrovasodilator in newborn piglets. Am J Physiol 1997;272:511-516.

42. Regriny $O$, Delagrange $P$, Scalbert E, Atkin- son J, Lartaaud-Idjouadiene I: Melatonin improves cerebral circulation security margin in rats. Am J Physiol Heart Circ Physiol 1998; 275:139-144. 



\section{Chapter}

\section{Neuroprotective effects of melatonin administered alone or in combination with topiramate in neonatal hypoxic-ischemic rat model}

Published as: Ozyener F, Cetinkaya M, Alkan T, Goren B, Kafa IM, Kurt MA, Koksal N. Neuroprotective effects of melatonin administered alone or in combination with topiramate in neonatal hypoxic-ischemic rat model. Restor Neurol Neuroscience 2012; 30: 435-444. 


\section{Abstract.}

\section{Purpose:}

The objective of this study was to compare the effects of two neuroprotective agents; melatonin, a free radical scavenger and topiramate, AMPA/kainate receptor antagonist, administered alone or in combination in neonatal hypoxic- ischemic model.

\section{Methods:}

After being anesthetized, 7-day-old pups underwent ischemia followed by exposure to hypoxia. The pups were divided into 4 groups in order to receive the vehicle, melatonin, topiramate and combination of topiramate and melatonin. These were administered intraperitoneally for three times; the first before ischemia, the second after hypoxia and the third 24 hours after the second dose. After sacrification, infarct volume and apoptosis were evaluated.

\section{Results:}

Percent infarcted brain volume was significantly reduced in rats which received drugs compared with those which received the vehicle. The number of TUNEL positive cells per unit area in hippocampus and cortex were markedly reduced in drug treated groups compared with control group. No significant differences were found regarding percent infarcted brain volume and number of TUNEL positive cells among drug-treated groups.

\section{Conclusions:}

Melatonin and topiramate, administered either alone or in combination significantly reduced the percent infarcted brain volume and number of TUNEL positive cells suggesting that these agents may confer benefit in treatment of infants with hypoxicischemic encephalopathy. 


\section{Introduction}

Hypoxic-ischemic (HI) brain damage is a major cause of neurological morbidity and mortality in neonates and results with sequelae such as cerebral palsy, mental retardation, epilepsy and learning disabilities (Vannucci and Hagberg, 2004). Although several mechanisms have been proposed to cause perinatal brain damage, most important of them include excitotoxicity induced by glutamate, influx of calcium $\mathrm{Ca}^{2+}$, production of free radicals and subsequent apoptosis and cell death (Jensen et al., 2003). The excessive glutamate release and overactivation of glutamate receptors contribute to the pathogenesis of $\mathrm{HI}$ brain injury. Three subtypes of ionotropic glutamate receptors namely, N-methyl-D-aspartate (NMDA) receptors, C-3-amino-hydroxy-5-methyl-4isoxazole propionic acid (AMPA) receptors and kainate (KA) receptors are involved in $\mathrm{HI}$ process (Lu et al.,1996). Excitotoxic injury occurs secondary to glutamate-triggered $\mathrm{Ca}^{2+}$ influx through NMDA channels, voltage-sensitive $\mathrm{Ca}^{2+}$ channels, and $\mathrm{Ca}^{2+}$-permeable AMPA/KA channels. Therefore, it was suggested that blocking these receptors might provide neuroprotective effects (Lee, Kim and Kim, 2000). Although, NMDA receptor antagonists such as MK801, magnesium, and memantine were shown to be neuroprotective in several animal models (Alkan et al., 2001; Turkyılmaz et al., 2002; Volbracht et al., 2006), there is growing recent recognition regarding the neuroprotective role of AMPA/KA antagonists such as topiramate on neonatal HI brain injury (Noh et al., 2006; Schubert et al., 2005; Sfaello et al., 2005).

Melatonin is an endogenously produced indoleamine which is primarily formed by the pineal gland in higher amount in adults than in neonates and is a potent free radical scavenger, and also an indirect antioxidant. Melatonin has the ability of distributing extensively in tissues, cells, subcellular compartments including brain since it crosses all morphophysiological barriers easily (Tan et al., 2005). A number of studies reported that melatonin could be used as a neuroprotective agent in neonatal $\mathrm{HI}$ brain injury (Carloni et al., 2008; Tutunculer et al., 2005; Welin et al., 2007). Topiramate, a well-tolerated and clinically widely-used antiepileptic drug provides neuroprotection by blocking AMPA/KA receptors and use-dependent sodium $\mathrm{Na}^{+}$channel in developing rat brain without serious side effects. Current studies, though limited in number, reported the probable neuroprotective effects of topiramate in neonatal $\mathrm{HI}$ brain damage (Noh et al., 2006; Schubert et al., 2005; Sfaello et al., 2005). It was also suggested that topiramate exhibited protective activities on developing white matter by shielding cultured preoligodendrocytes from AMPA/kainate receptor-mediated damage (Follett et al., 2004). Furthermore, topiramate was shown to be protective against hyperoxic brain injury in the developing brain (Kurul et al., 2009).

It was reported that intervening one particular pathway would not completely prevent $\mathrm{HI}$ brain damage, therefore, using combination of drugs interfering at different levels of $\mathrm{HI}$ cascade was suggested to have a greater neuroprotective effect compared with a single therapy intended for a single pathway (Peeters and van Bel, 2001). 
Therefore, we aimed to investigate the effects of two neuroprotective agents, having two different actions on $\mathrm{HI}$ cascade, topiramate as an antiglutamatergic agent and melatonin as a free radical scavenger, either solely or in combination in neonatal $\mathrm{HI}$ rat model. We expected that the combination would yield a greater therapeutic benefit compared to that obtained with either agent alone.

\section{Materials and methods}

\subsection{Animals}

This study was performed in accordance with the guidelines provided by the Experimental Animal Laboratory and all experimental protocols and procedures were approved by the Animal Care and Use Committee of the Uludag University, Faculty of Medicine. A modified Levine-Rice preparation was used as a model for perinatal HI brain injury as previously reported (Vannucci and Vannucci, 1997). Dated pregnant SpragueDawley rats were housed in individual cages under standard conditions with free access to water and laboratory chow. The day of spontaneous vaginal delivery was considered postnatal day 1 (PND1) for the pups and a total of 80 offsprings were reared with their dams until initiation of experiments on day 7 (PND7). Rat pups of either sex weighing 12$16 \mathrm{~g}$ were used in the experiments.

\subsection{Study design}

PND7 rats $(n=80)$ that were subjected to hypoxia- ischemia were divided randomly and equally into 4 groups and injected intraperitoneally (IP) with the following: vehicle saline, $0.1 \mathrm{ml}$; melatonin (Mel, $20 \mathrm{mg} / \mathrm{kg}$ ), topiramate (TPM, $30 \mathrm{mg} / \mathrm{kg}$ ) and a combination of melatonin and topiramate [(Mel, $20 \mathrm{mg} / \mathrm{kg})+(T P M, 30 \mathrm{mg} / \mathrm{kg})]$. Treatments were given three times; the first being just before ischemia, the second after hypoxia and the third 24 hours after the second dose. The schematic diagram of the experimental protocol is shown in Fig. 1.

The dose of topiramate used in our study $(30 \mathrm{mg} / \mathrm{kg}$ ) was similar to those used in previous experimental models of neonatal HI brain injury (Follett et al., 2004; Liu et al., 2004). Similarly, melatonin dose $(20 \mathrm{mg} / \mathrm{kg}$ ) was also within the upper range of melatonin doses which were reported to exhibit the greatest neuroprotection in previous studies (ranging 5 to 20 mg/kg) (Carloni et al., 2008; Tutunculer et al., 2005; Welin et al., 2007).

Topiramate (Sigma-Aldrich, USA) was provided as a powder and a solution was prepared by dissolving in phosphate buffered saline (PBS) according to the instructions of the manufacturer as the final solution included a concentration of topiramate was 30 $\mathrm{mg} / \mathrm{kg}$ per microliter. Melatonin (Sigma, St. Louis, MO) was dissolved freshly in absolute ethanol and liquidized in isotonic sodium chloride $(0.9 \% \mathrm{NaCl})$ to obtain a final concentration of $1: 10$ solution form. 


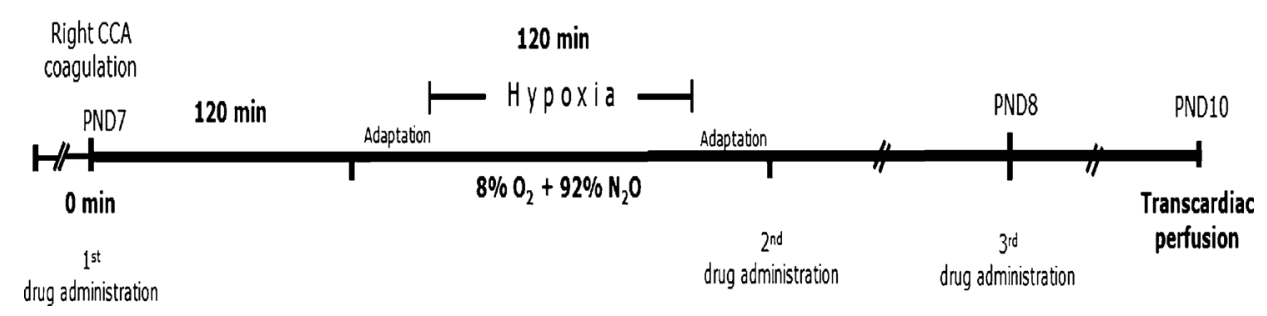

Fig. 1. Schematic diagram of experimental steps.

\subsection{Induction of $\mathrm{HI}$ brain injury and treatment groups}

Pups were anesthetized lightly with inhaled isoflurane, the right carotid artery was accessed through a longitudinal neck incision, isolated from the nerve and vein, and permanent surgical ligation with 5.0 surgical silk plus coagulation of carotid artery was performed. This procedure lasted no longer than 3 minutes and it was performed at room temperature. Animals exhibiting bleeding during ligation or respiratory arrest due to anesthesia were excluded from the study. After closure of the neck incision, pups were allowed to recover for 15 minutes under a heating lamp and then returned to the nest temperature. Following a 2 hour period of nesting, the pups were placed into chambers that were partially submerged in a $37^{\circ} \mathrm{C}$ water bath to maintain a constant thermal environment and exposed to hypoxia with warm humidified 8\%/92\% oxygen/nitrogen mixture for 2 hours. Upon retrieval from the hypoxia chambers, the pups were allowed to recover for $72 \mathrm{~h}$ with their dams. Following deep isoflurane anesthesia, cardiac perfusion was performed with phosphate buffer solution containing 4\% paraformaldehyde and their brains were removed for evaluation of hypoxic damage and immunohistochemical analysis.

\subsection{Measurement of infarcted volume}

Infarct volume was evaluated as previously described (Vannucci et al., 1999). Cardiac perfusion was performed 72 hours after hypoxia (PND10). Pups were sacrificed immediately ( $n=6$, each group), and the brains were quickly removed, placed in ice-cold saline for $5 \mathrm{~min}$, and $2 \mathrm{~mm}$-thick coronal sections were obtained starting from the frontal pole using neonatal brain matrix (Ted Pella, CA, USA). The slices were incubated in $2 \% 2-$ , 3-, 5-triphenyl-tetrazolium chloride (TTC, dissolved in saline) for $15 \mathrm{~min}$ at $37^{\circ} \mathrm{C}$, fixed by immersion in 10\% formalin, and images acquired with Sony Cybershot DSC-F717 digital camera attached to a Nikon 4S-2 Alphaphot light microscope. The white areas of infarct were quantified using an image-analysis software (Scion Corporation version 4.02, NIH, Bethesda, MD, USA). The mean infarct volume per slice was obtained from the product of the average thickness of a slice $(2 \mathrm{~mm})$ and the area of infarct in that section. 


\subsection{Immunohistochemistry}

Rats were anesthetized by inhaled isoflurane and perfused with $4 \%$ paraformaldehyde in $0.1 \mathrm{M}$ PBS ( $\mathrm{pH}$ 7.4). Their brains were then removed, post-fixed overnight, embedded in paraffin and $5 \mu \mathrm{m}$ coronal sections were obtained using a microtome. Sections were chosen according to systematic random sampling procedure, which were then mounted onto poly-L-lysine-coated slides (Histobond adhesion slides, Marienfeld, Germany). In situ cell death detection POD kit (Roche Molecular Bochemicals, 11684817910, Germany) was used for TUNEL technique [terminal deoxynucleotidyl transferase (TdT)- mediated deoxyuridine triphosphate (dUTP)-biotin nick end-labeling] and the sections stained according to the manufacturer's protocol for paraffin embedded tissues. Briefly, sections were heated at $60^{\circ} \mathrm{C}$ for 45 min followed by washing in xylene and rehydration through graded series of alcohol (ethanol; 100\%, 95\%, 80\%, 70\%, 40\%) and washed in distilled water. They were then incubated in $20 \mu \mathrm{g} / \mathrm{mL}$ proteinase $\mathrm{K}$ solution (Roche Applied Sciences, 031158444001, Germany) for $20 \mathrm{~min}$ at room temperature (RT), immersed in $3 \% \mathrm{H}_{2} \mathrm{O}_{2}$ in methanol for 10 minutes. Permeabilization with Triton X (Sigma-Aldrich, T9284, USA) for 10 min was performed on ice. The sections were then incubated with 35 $\mu$ I TUNEL reaction mixture at $37^{\circ} \mathrm{C}$ in a humidified chamber for 1 hour followed by converter-POD treatment at $37^{\circ} \mathrm{C}$ for $30 \mathrm{~min}$. Diamino benzidine tetrahydrochlioride (DAB) (50 $\mu$ 1:9, diaminobenzidine/metal concentrate: peroxidase buffer, Roche Applied Sciences, 11718096001, Germany) was used as the chromogene and the slides were counterstained with hematoxylin (Harris's hematoxylin, Sigma-Aldrich, HHS16, USA), and coverslipped (DPX mountant, Fluka, 44581, USA). $35 \mu \mathrm{l}$ DNase $(200 \mu \mathrm{g} / \mathrm{ml}$ DNase I (Roche Applied Sciences, Germany) in DNase buffer (40 mM Tris HCl, pH 7.610 $\mathrm{mM} \mathrm{NaCl}, 6 \mathrm{mM} \mathrm{MgCl}_{2}, 10 \mathrm{mMCaCl}_{2}$ ) was used for positive controls for $10 \mathrm{~min}$ at $37^{\circ} \mathrm{C}$, while, alternate sections were incubated in the absence of TUNEL reaction mixture for negative controls. TUNEL positive cells with characteristic nuclear morphological changes including chromatin condensation, hypersegmentation of nuclear chromatin and shrunken (pyknotic) appearance were counted on using an ocular micrometer grid randomly on the CA1, CA3 and dentate gyrus (DG) areas of hippocampus and cerebral cortex (especially parietal cortex).

Immunohistochemistry staining for caspase-3 was performed using the Avidin Biotin Complex $(A B C)$ technique. Sections were heated at $60^{\circ} \mathrm{C}$ for $1 \mathrm{hr}$ followed by washing in xylene and rehydration through graded series of alcohol (ethanol; 100\%, 95\%, 80\%, 70\%, $40 \%$ ) and washed in distilled water. They were then placed in $10 \mathrm{mM}$ citrate buffer at $\mathrm{pH}$ 6.0 and treated with $750 \mathrm{~W}$ microwave for $10 \mathrm{~min}$ for antigen retrieval and cooled for 20 min at RT. Then slides were immersed in $3 \% \mathrm{H}_{2} \mathrm{O}_{2}$ for 10 minutes and incubated in $10 \%$ goat serum in tris buffer $(0.01 \mathrm{mM}$ tris $\mathrm{HCl}, \mathrm{pH} 7.4,1 \mathrm{mM} \mathrm{CaCl}, 1 \mathrm{mM} \mathrm{MgCl}$, \% 0.1 Triton $\mathrm{X}-100)$ for $30 \mathrm{~min}$ at RT. After blocking, slides were stained with Caspase-3 (1: 100; Chemicon, AB3623-rabbit, USA) antibody which is diluted in same blocking buffer, overnight at $4^{\circ} \mathrm{C}$. Secondary antibody (biotinylated anti-rabbit IgG, Vectastain Elite $A B C$ 
Kit, USA) was applied for $1 \mathrm{hr}$ at RT. Sections were then treated with $100 \mu \mathrm{l}$ of avidine biotin complex (ABC Vectastain Elite ABC Kit, USA) and finally visualized using DAB (50 $\mu$ l 1:9, diaminobenzidine/metal concentrate: peroxidase buffer) and counterstained with hematoxylin.

\subsection{Cell count}

A total of at least 200 cells counted in 7 fields were chosen randomly at 400x magnification for evaluation of TUNEL positive cells' index with respect to their morphological characteristics. In addition, all positive cells for Caspase-3 immunoreactivity were counted at 200x magnification (Kafa et al., 2010).

\subsection{Statistical analysis}

Data regarding the "percent infarcted volume" obtained by TTC staining were analyzed using "Kruskal-Wallis one-way ANOVA" between groups ( $n=12$ for each group). Further analysis between groups were made using "Mann-Whitney U Test" if significant differences were found by ANOVA. TUNEL-positive cell counts were also analyzed using "Kruskal-Wallis one-way ANOVA" and "Mann- Whitney U Test". Sigma-stat 3.0 and SPPS 16.0 programs were used for the statistical tests and significance assumed at $95 \%$ confidence $(p<0.05)$.

\section{Results}

Melatonin (17.4 $\pm 5.6, p<0.001)$, Topiramate $(25.4 \pm 3.0, p<0.01)$ and Melatonin + Topiramate $(14.0 \pm 5.7, \mathrm{p}<0.001)$ administration significantly reduced "the percent infarcted volume", compared to control ischemic animals (49.1 \pm 7.6$)$ (Figs. 2, 3). There was no statistically significant difference between Melatonin, Topiramate and Melatonin + Topiramate treated groups ( $p>0.05)$. TUNEL stain showed markedly reduced numbers of TUNEL positive cells at CA1, CA3 and DG areas of hippocampus at drug treated groups $(p<0.001)$. The mean number of TUNEL positive cells at CA1, CA3 and DG areas of hippocampus at control group were $33.2 \pm 1.9,26.7 \pm 2.0,18.9 \pm 3.4$, whereas these were significantly reduced as $2.4 \pm 0.7,1.3 \pm 0.1,4.3 \pm 1.1$ in Melatonin group, $1.6 \pm 0.2,1.4$ $\pm 0.2, \pm 0.1$ in Topiramate group and $1.5 \pm 0.3,1.3 \pm 0.2, \pm 0.2$ in Melatonin + Topiramate group, respectively (all $p<0.001$, in study groups compared with control group). No statistically significant difference was found for TUNEL-positive cell count between drug treated groups ( $p>0.05$ ) (Fig. 4). There were also small number of neurons stained positively in cortex at Melatonin, Topiramate and Melatonin + Topiramate groups (4.91 $\pm 0.44,2.19 \pm 0.13,2.15 \pm 0.25$, respectively) compared to control ischemic animals $(33.78 \pm 1.25)$ at unit area $(p<0.001)$. However, there was no significant difference between three treatment groups ( $p>0.05$ ) (Fig. 5). In addition, the number of caspase-3 
positive cells was significantly lower in drug treated groups compared with the control group ( $p<0.001$ ) (Figs. 6 and 7); again no significant difference was found among treated groups in terms of caspase-3 immunoreactivity ( $p>0.05)$.

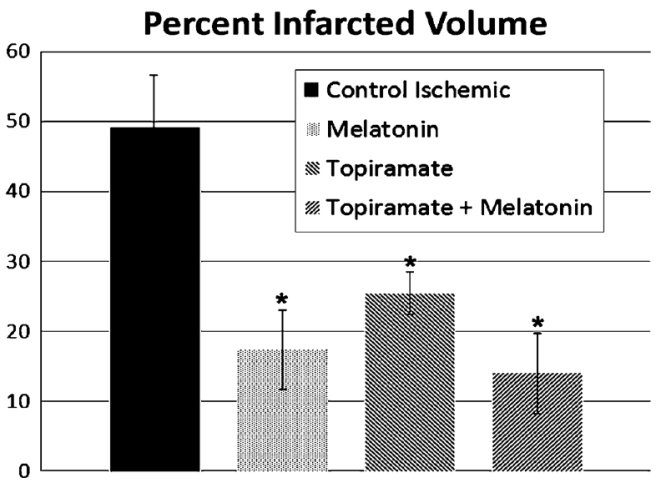

Fig. 2. Percent infarcted volume of brains of pups in each group. $* p<0.05$ compared to control.
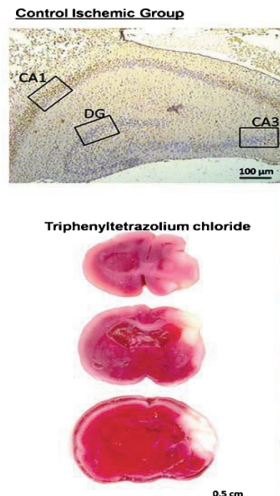

Topiramate Group

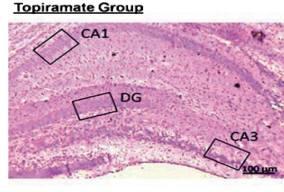

Triphenyttetrazolium chloride

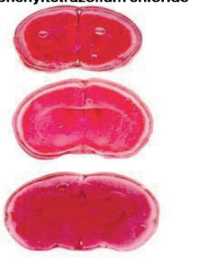

$0.5 \mathrm{~cm}$
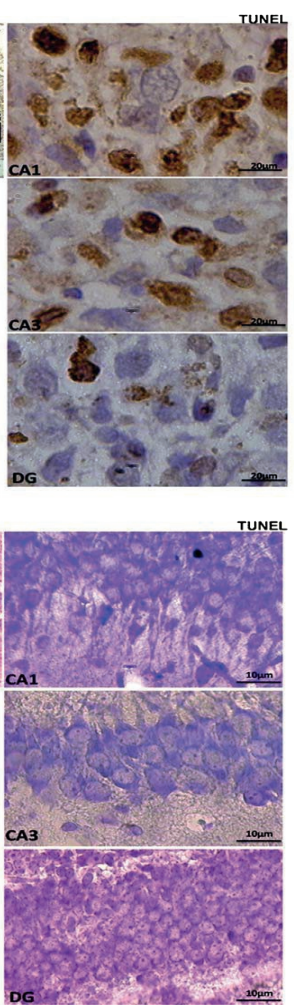

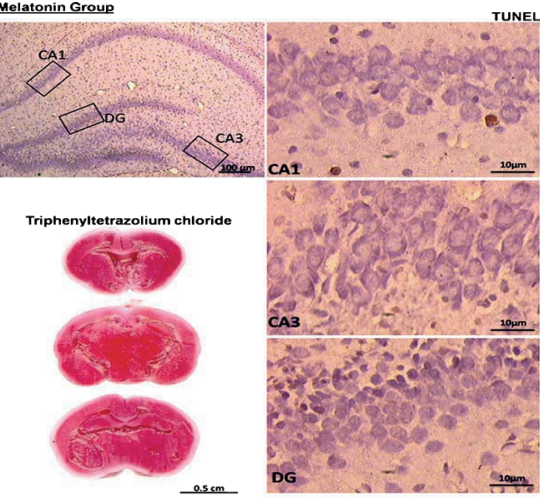

Topiramate + Melatonin Group
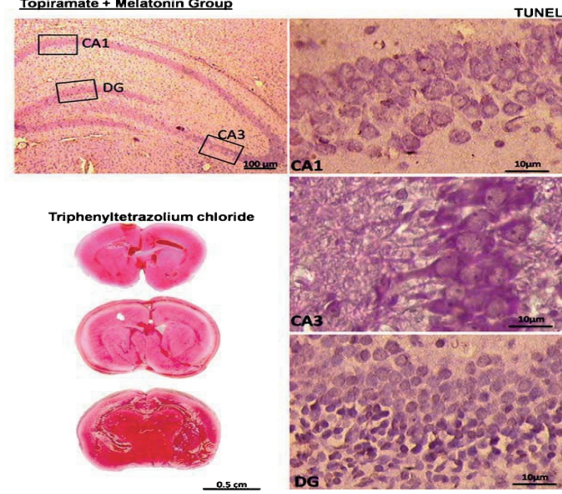

Fig. 3. Figure showing TTC stained coronal sections from each experimental group with representative images of TUNEL staining in hippocampus. 
TUNEL

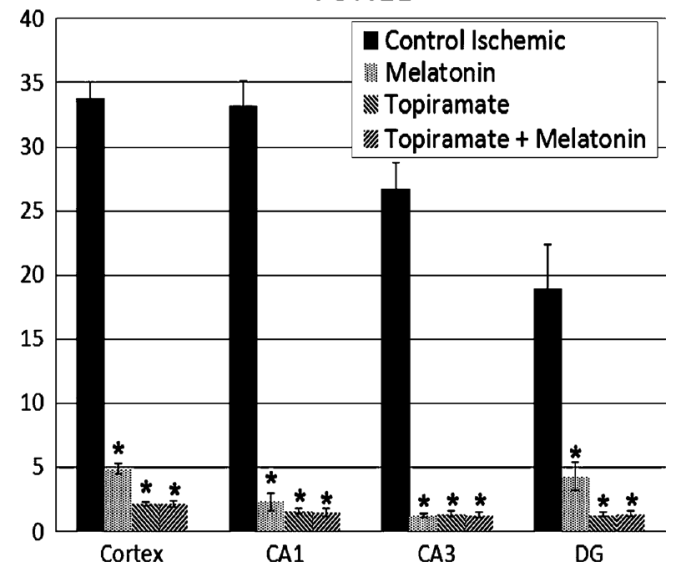

Fig. 4. TUNEL positive cell count per unit area of cortex and hippocampus in each experimental group. ${ }^{*} p<0.05$ compared with control group.
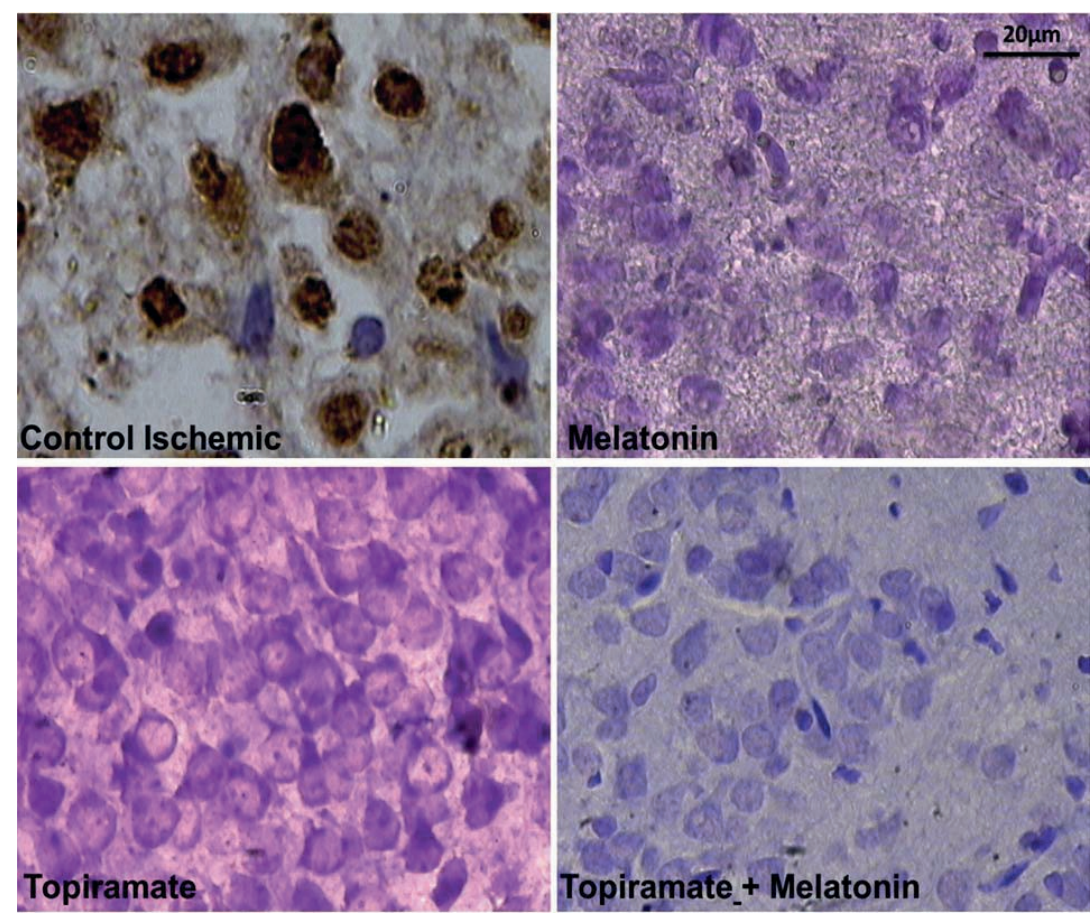

Fig. 5. Representative images of TUNEL staining in cortex for each group. 


\section{Caspase-3}

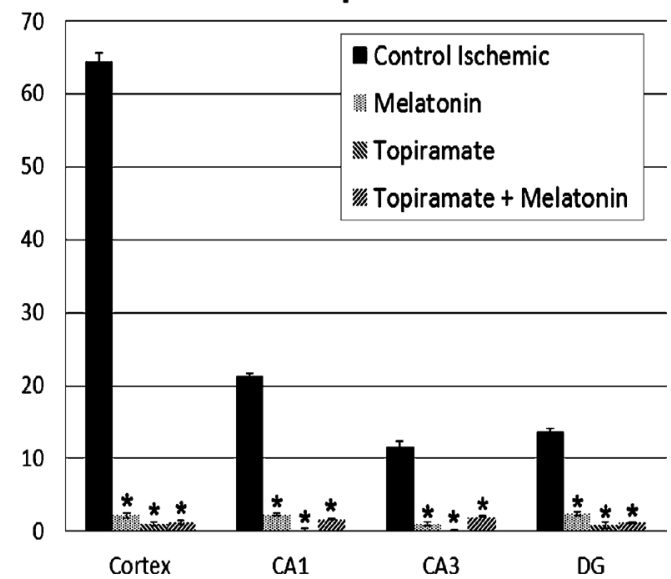

Fig. 6. Caspase-3 positive cell count per unit area of cortex and hippocampus in each experimental group. *p $<$ 0.05 compared with control group.

\section{CASPASE 3}

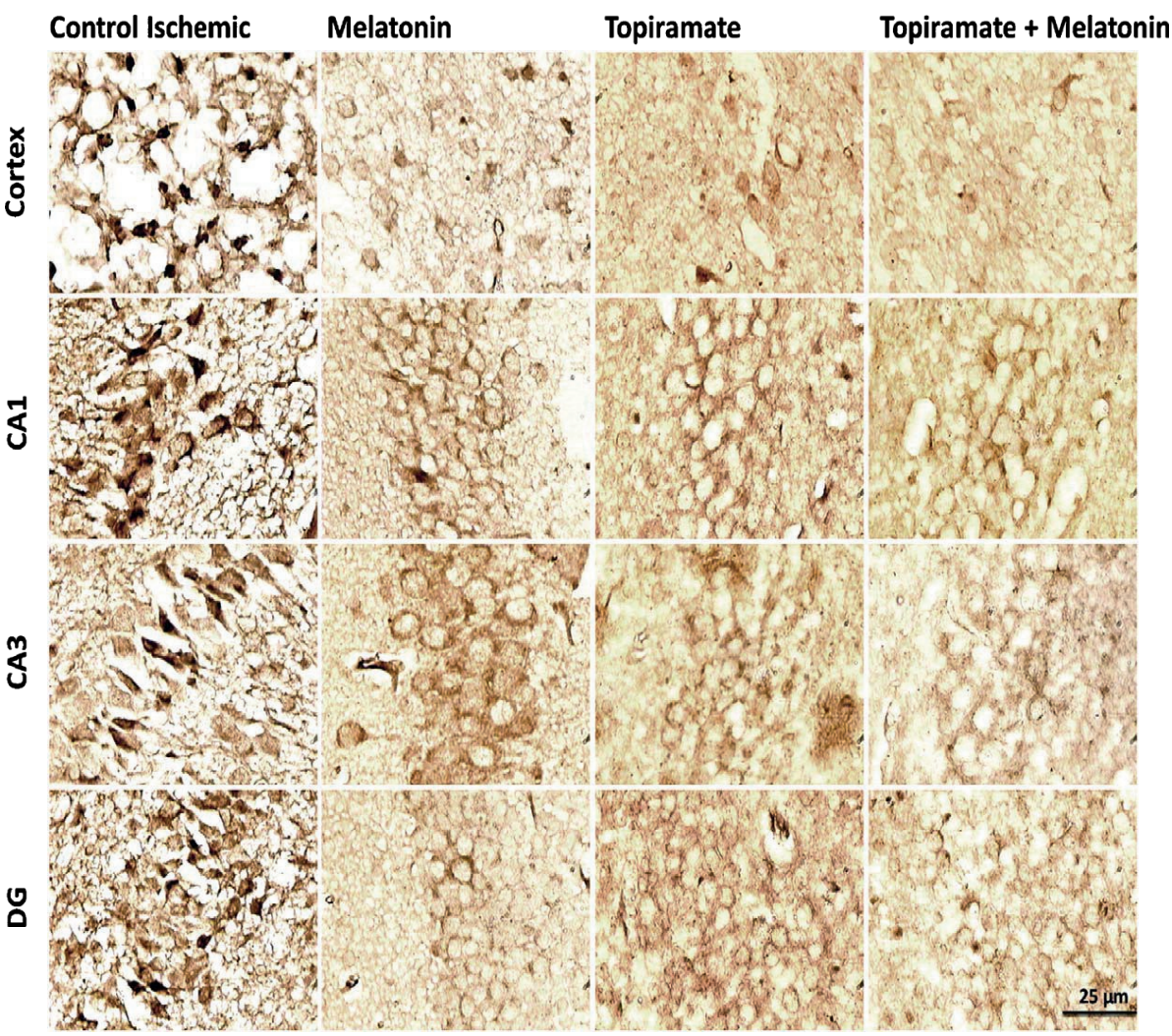

Fig. 7. Caspase-3 immunoreactivity in cortex and hippocampal areas of each group. 


\section{Discussion}

These data indicate that both melatonin and topiramate, two different agents acting on different levels of $\mathrm{HI}$ cascade significantly reduced the percent infarcted volume and apoptosis after either single or combined administration in neonatal $\mathrm{HI}$ rat model. Although the reduction in infarct volume was greater after the combined therapy compared with the single use of these agents, the difference was not statistically significant. Topiramate has been approved to be used as an antiepileptic agent in children older than 2 years of age and was recently suggested that it might provide neuroprotection by blocking AMPA/KA receptors in neonatal HI brain injury (Glauser, 1999; Noh et al., 2006; Schubert et al., 2005; Sfaello et al., 2005). Noh et al. (2006) reported that IP or peroral topiramate pre-treatment significantly reduced the brain damage and subsequent cognitive impairment induced by hypoxia- ischemia in neonatal rats. Similarly, Sfaello et al. (2005) found that topiramate had dose-dependent and long lasting neuroprotection in the excitotoxic newborn mouse model and suggested that topiramate provided neuroprotection by increasing survival of pre-oligodendrocytes, decreasing neuronal apoptosis, inhibiting microglial activation and astrogliosis, and decreasing seizure activity. Lately, topiramate was found to be neuroprotective in neonatal hyperoxic brain injury model, too (Kurul et al., 2009). In this study, we found that topiramate administered just before ischemia and right after hypoxia significantly decreased brain infarct volume and neuronal apoptosis.

Free oxygen radicals generated during and after hypoxia-ischemia also play an important role in the pathogenesis of neonatal $\mathrm{HI}$ brain injury. Since melatonin could cross all morphophysiological barriers, as a potent free radical scavenger and an indirect antioxidant, it was presumed to be a neuroprotective agent in neonatal $\mathrm{HI}$ brain damage (Carloni et al., 2008; Tutunculer et al., 2005; Welin et al., 2007). Melatonin also significantly reduced inflammation and cell death following fetal umbilical cord occlusion (Welin et al., 2007). Recently, melatonin, administered before and after hypoxia, exhibited significant neuroprotective effect and lessened behavioral problems and learning deficits in long-term follow-up (Carloni et al., 2008). In adult rats, melatonin that was given after ischemia was reported to lead long-term preservation of both the neural substrate, and the capability for integration of spatial learning and memory, mainly dependent on a normal hippocampal functioning (Letechipıa-Vallejo et al., 2007). In the present study, administration of melatonin demonstrated a significant neuroprotective effect in a neonatal rat model of $\mathrm{HI}$ brain damage.

Combination of drugs intervening at different levels of the $\mathrm{HI}$ cascade instead of a single therapy aiming at a single pathway was suggested to have a greater neuroprotective effect (Peeters and van Bel, 2001). In a recent review, it was proposed that the best outcome in neonatal $\mathrm{HI}$ brain injury might be achieved with a multimodal therapeutic approach such as a combination of glutamate receptor antagonists with antioxidants and hypothermia (Fatemi et al., 2009). Subsequently, it was also suggested 
that combined neuroprotective therapies might be necessary for increasing the therapeutic time window for protection and repair in neonatal HI brain damage (Gonzalez and Ferriero, 2009). Therefore, we aimed to evaluate the neuroprotective effects of a combination of melatonin and topiramate in neonatal HI brain injury.

In our previous study, we investigated the neuroprotective effect of melatonin as part of a combination therapy in neonatal HI rat model (Cetinkaya et al., 2011). That study showed that magnesium sulfate and melatonin, two agents also acting at different stages of $\mathrm{HI}$ brain damage, administered either alone or in combination significantly reduced the percent infarcted brain volume and TUNEL positivity. It was, therefore, suggested that these agents might confer possible benefit in treatment of infants with $\mathrm{HI}$ encephalopathy (HIE). Similarly, the results of the present study show a significant neuroprotective effect of melatonin and topiramate combination therapy.

Limited number of studies evaluated the neuroprotective efficacy of topiramate in combination with other agents in neonatal HI brain injury. Early post-HI administration of topiramate in combination with hypothermia resulted with sustained reduction in brain injury and improved functional performance in a neonatal rat stroke model (Liu et al., 2004). Recently, combination of memantine, a non-competitive NMDA receptor blocker with topiramate also safely and effectively reduced brain damage compared with each agents given alone in neonatal HI brain injury (Liu et al., 2009). In a recent study, oral topiramate (either low or high dose) in combination with hypothermia (either mild or deep) showed similar neuroprotective effects and the authors concluded that oral topimarate could be used clinically in neonates with asphyxia in order to benefit its possible additive neuroprotective effect (Filippi et al., 2010). Although our results are in agreement with these data, however, the neuroprotective effect of combination therapy was not statistically different from that exhibited by each agent on its own. This insignificance could have resulted from administration time and dose of the drug or, alternatively, with the design of studies. Recent evidence suggested that therapies might be combined to enhance the protective and reperative processes and administration time seemed to be the crucial point for increasing the success of the combined therapies. Also, as the injury might evolve for a long period with different mechanistic phases, combined therapies aiming at these temporally evolving targets might also be administered over long periods of time (Gonzalez and Ferriero, 2009). Therefore, the insignificance of combined therapy in our study might be associated with the relatively short duration of therapies. It might also be related with the administration time of both drugs. Rather than administering both agents simultaneously, as we did, it could be possible to achieve significant results by giving topiramate first as an antiglutamatergic agent, and then melatonin as an antioxidant agent.

In this study, we especially evaluated these two agents as both melatonin and topiramate could be used in neonates clinically. Melatonin was used safely and efficiently in treatment of sepsis, bronchopulmonary dysplasia and asphyxia in newborn infants (Fulia et al., 2001; Gitto et al., 2001; Gitto et al., 2004). Topiramate was also started to be 
choice of therapy both in infants with neonatal seizures and neonatal HIE (Filippi et al., 2010; Silverstein and Ferriero, 2008). Therefore, the results of this study seem to support the idea of using these agents for the clinical treatment of infants with HIE.

The limitation of this research was that we could not evaluate other indices of HI injury such as cellular regeneration and inflammation. Moreover, due to technical shortcomings, neuroprotective effects of both agents were evaluated for a brief period of time. Consequently, we were unable to observe possible long term neuroprotective effects of melatonin and topiramate. Therefore, we suggest that long term behavioral studies are also warranted to assess the potential synergistic effect of the combined therapies.

In conclusion, both melatonin and topiramate, two agents acting on different stages of hypoxia-ischemia, used either alone or in combination, significantly decreased the percent infarcted area, apoptotic cell death in neonatal $\mathrm{HI}$ rat model. Although the combination therapy had a superior effect on decreasing percent infracted area and apoptotic cell count compared with single use of these two agents, this effect was not significantly superior to mono therapies. Therefore, it would be reasonable to investigate the different doses and different application times of these agents as combination therapy for a significantly and more effective neuroprotection. The data provided here may render a valuable reference source for future clinical investigation and these two agents may be used safely in combination for clinical treatment of infants with HIE.

\section{Acknowledgments}

This study was supported by Uludag University Scientific Research Project with a project number T-2800/60.

\section{Conflict of interest statement}

Authors state no conflict of interest. 


\section{References}

1. Alkan, T., Kahvecı, N., Buyukuysal, L., Korfali, E. \& Ozluk, K. (2001). Neuroprotective effects of MK801 and hypothermia used alone or in combination in hypoxic-ischemic brain injury in neonatal rats. Arch Physiol Biochem, 109(2), 135-144.

2. Carlonı, S., Perrone, S., Buonocore, G., Longini, M., Proietti, F. \& Balduini, W. (2008). Melatonin protects from the long-term consequences of a neonatal hypoxic-ischemic brain injury in rats. J Pineal Res, 44(2), 157-164.

3. Cetinkaya, M., Alkan, T., Ozyener, F., Kafa, I.M., Kurt, A.M. \& Koksal, N. (2011). Possible neuroprotective effects of magnesium sulfate and melatonin as both pre- and post-treatment in neonatal hypoxic-ischemic rat model. Neonatology, 99(4), 302-310.

4. Fatemi, A., Wilson, M.A. \& Johnston, M.V. (2009). Hypoxic- ischemic encephalopathy in the term infant. Clin Perinatol, 36(4), 835-858.

5. Filippi, L., Poggi, C., La Marca, G., Furlanetto, S., Fiorini, P., Cavallaro, G., Plantulli, A., Donzelli, G. \& Guerrini, G. (2010). Oral topiramate in neonates with hypoxic ischemic encephalopathy treated with hypothermia: A safety study. J Pediatr, 157(3), 361-366.

6. Follett, P.L., Deng, W., Dai, W., Talos, D.M., Massillon, L.J., Rosenberg, P.A., Volpe, J.J. \& Jensen, F.E. (2004). Glutamate receptor-mediated oligodendrocyte toxity in periventricular leukomalacia: A protective role for topiramate. J Neurosci, 24(18), 4412-4420.

7. Fulia, F., Gitto, E., Cuzzocrea, S., Reiter, R.J., Dugo, L., Gitto, P., Barberi, S., Cordaro, S. \& Barberi, I. (2001). Increased levels of malondialdehyde and nitrite/nitrate in the blood of asphyxiated newborns: Reduction by melatonin. J Pineal Res, 31(1), 343- 349.

8. Gitto, E., Karbownik, M., Reiter, R.J., Tan, D.X., Cuzzocrea, S., Chiurazzi, P., Cordaro, S., Corona, G., Trimarchi, G. \& Barberi, (2001). Effects of melatonin treatment in septic newborns. Pediatr Res, 50(6), 756-760.

9. Gitto, E., Reiter, R.J., Amodio, A., Romeo, C., Cuzzocrea, E., Sabatino, G., Buonocore, G., Cordaro, V., Trimarchi, G. \& Barberi, I. (2004). Early indicators of chronic lung disease in preterm infants with respiratory distress syndrome and their inhibition by melatonin. J Pineal Res, 36(4), 250-255.

10. Glauser, T.A. (1999). Topiramate. Epilepsia, 40(S5), S71-S80.

11. Gonzalez, F.F. \& Ferriero, D.M. (2009). Neuroprotection in the newborn infant. Clin Perinatol, 36(4), 859880 .

12. Jensen, A., Garnier, Y., Middelanis, J. \& Berger, R. (2003). Perinatal brain damage-from pathophysiology to prevention. Eur J Obstet Gynecol Reprod Biol, 110(S1), S70-S79.

13. Kafa, I.M., Uysal, M., Bakirci, S. \& Kurt, M.A. (2010). Sepsis induces apoptotic cell death in different regions of the brain in a rat model of sepsis. Acta Neurobiol Exp, 70(3), 246-260.

14. Kurul, S.H., YIS, U., Kumral, A., Tug yan, K., Cilaker, S., Kolatan, E., Yılmaz, O. \& Genc, S. (2009). Protective effects of topiramate against hyperoxic brain injury in the developing brain. Neuropediatrics, 40(1), 22-27.

15. Lee, S.R., Kim, S.P. \& Kim, J.E. (2000). Protective effects of topiramate against hippocampal neuronal damage after global ischemia in the gerbils. Neurosci Lett, 281(2-3), 183-186.

16. Letechipı-Vallejo, G., Lopez-Loeza, E., Espinoza-Gonzalez, V., Gonzalez-Burgos, I., Olvera-Cortes, M.E., Moralı, G. \& Cervantes, M. (2007). Long-term morphological and functional evaluation of the neuroprotective effects of post-ischemic treatment with melatonin in rats. J Pineal Res, 42(2), 138-146.

17. Liu, C., Lin, N., Wu, B. \& Qiu, Y. (2009). Neuroprotective effect of memantine combined with topiramate in hypoxic-ischemic brain injury. Brain Res, 1282, 173-182.

18. Liu, Y., Barks, J.D., Xu, G. \& Silverstein, F.S. (2004). Topiramate extends the therapeutic window for hypothermia-mediated neuroprotection after stroke in neonatal rats. Stroke, 35(6), 1460-1465.

19. Lu, Y.M., Yin, H.Z., Chiang, J. \& Weiss, J.H. (1996). Ca2+- permeable AMPA/kainate and NMDA channels: High rate of Ca2+ influx underlies potent induction of injury. J Neurosci, 16(17), 5457-5465.

20. Noh, M.R., Kım, S.K., Sun, W., Park, S.K., Choi, H.C., Lim, J.H., Kim, I.H., Kim, H.J., Kim, H. \& Eun, B.L. (2006). Neuroprotective effect of topiramate on hypoxic ischemic brain injury in neonatal rats. Exp Neurol, 201(2), 470-478. 
21. Peeters, C. \& Van Bel, F. (2001). Pharmacotherapeutical reduction of the post-hypoxic-ischemic-brain injury in the newborn. Biol Neonate, 79(3-4), 274-280.

22. Schubert, S., Brandl, U., Brodhun, M., Ulrich, C., Spaltmann, J., Fiedler, N. \& Bauer, R. (2005). Neuroprotective effects of topiramate after hypoxia-ischemia in newborn piglets. Brain Res, 1058(1-2), 129-136.

23. Sfaello, I., Baud, O., Arzimanoglou, A. \& Gressens, P. (2005). Topiramate prevents excitotoxic damage in the newborn rodent brain. Neurobiol Dis, 20(3), 837-848.

24. Silverstein, F.S. \& Ferriero, D.M. (2008). Off-label use of antiepileptic drugs for treatment of neonatal seizures. Pediatr Neurol, 39(2), 77-79.

25. Tan, D.X., Manchester, L.C., Terron, M.P., Flores, L.J. \& Reiter, R.J. (2007). One molecule many derivatives: A never-ending interaction of melatonin with reactive oxygen and nitrogen species? J Pineal Res, 42(1), 2842.

26. Tomas-Zapıco, C. \& Coto-Montes, A. (2005). A proposed mechanism to explain the stimulatory effect of melatonin on antioxidative enzymes. J Pineal Res, 39(2), 99-104.

27. Turkyılmaz, C., Turkyılmaz, Z., Atalay, Y., Soylemezoglu, F. \& Celasun, B. (2002). Magnesium pre-treatment reduces neuronal apoptosis in newborn rats in hypoxia-ischemia. Brain Res, 955(1-2), 133-137.

28. Tutunculer, F., Eskıocak, S., Basaran, U.N., Ekukulu, G., Ayvaz, S. \& Vatansever, U. (2005). The protective role of melatonin in experimental hypoxic brain damage. Pediatr Int, 47(4), 434- 439.

29. Vannuccl, R.C., Connor, J.R., Mauger, D.T., Palmer, C., Smith, M.B., Towfighi, J. \& Vannucci, S.J. (1999). Rat model of perinatal hypoxic-ischemic brain damage. J Neurosci Res, 55(2), 158- 163.

30. Vannuccl, R.C. \& Vannuccl, S.J. (1997). A model of perinatal hypoxic-ischemic brain damage. Ann N Y Acad Sci, 835, 234- 249.

31. Vannucci, S.J. \& Hagberg, H. (2004). Hypoxia-ischemia in the immature brain. J Exp Biol, 207(pt 18), 31493154.

32. Volbracht, C., Van Beek, J., Zhu, C., Blomgren, K. \& Leist, M. (2006). Neuroprotective properties of memantine in different in vitro and in vivo models of excitotoxicity. Eur J Neurosci, 23(10), 2611-2622.

33. Welın, A.K., Svedın, P., Lapatto, R., Sultan, B., Hagberg, H., Gressens, P., Kjellmer, I. \& Mallard, C. (2007). Melatonin reduces inflammation and cell death in white matter in the mid-gestation fetal sheep following umbilical cord occlusion. Pediatr Res, 61(2), 153-158. 

Chapter

\section{Discussion and conclusions}


In this thesis, we tested different approaches to prevent and or treat severe neonatal diseases. We studied medical interventions that exploit different mechanisms of multifactorial neonatal conditions in appropriate animal models. In particular, we tested not only postnatal interventions but also prenatal interventions since many neonatal diseases have an antenatal origin that may be aggravated postnatally (Gantert et al., 2010). Therefore, we hypothesize that novel appropriate perinatal interventions for prevention and/or treatment of severe morbidities with multifactorial pathogenesis in experimental models may be translated into clinical experience in future. This may also lead to a decrease in the frequency and severity of these morbidities.

BPD is a good example for the complexity of perinatal disease. BPD has a multifactorial etiology including preterm birth, lower gestational age and birth weight, need for supplemental oxygen and ventilator support, oxygen toxicity, decreased host antioxidant defenses, patent ductus arteriosus, and pre- and postnatal infections. In addition to the increase in the number of surviving preterm infants associated with the improvement in neonatal care, the incidence of BPD did not show a significant decrease (Stoll et al., 2015). This may also be associated with the lack of effective prevention and treatment interventions (Poets and Lorenz, 2018). Although postnatal steroids, caffeine, azithromycin, and vitamin A were reported to confer benefit for prevention of BPD in ELBW infants, none of them provides cure due to the multifactorial pathogenesis of the disease (Poets and Lorenz, 2018). Because postnatal steroids have significant neurological adverse effects for this high-risk patient population, newer strategies and pharmacological approaches are required in order to decrease BPD incidence and severity without induction of comorbidities (Jensen et al., 2015).

\section{Role of epigenetic mechanisms in development of BPD}

Epigenetics can be defined as the partially heritable changes in gene function that occur without any change in deoxyribonucleic acid (DNA) sequence through changes in chromatin structure (Li et al., 2007). DNA methylation, histone modification and gene silencing by non-coding RNAs constitute the main epigenetic modifications (Figure 1) (Fonseca et al., 2018). These epigenetic mechanisms were suggested to play a major role in the susceptibility to both the pathogenesis and to the progression of several inflammatory respiratory diseases (Barnes, 2009). Epigenetic mechanisms were reported to be influenced by specific genetic polymorphisms, nutritional effects, inflammation, exposure to toxins and ventilator treatments in critically ill infants and were suggested to have profound effects on lung development that contribute to BPD development (Merritt et al., 2012). 


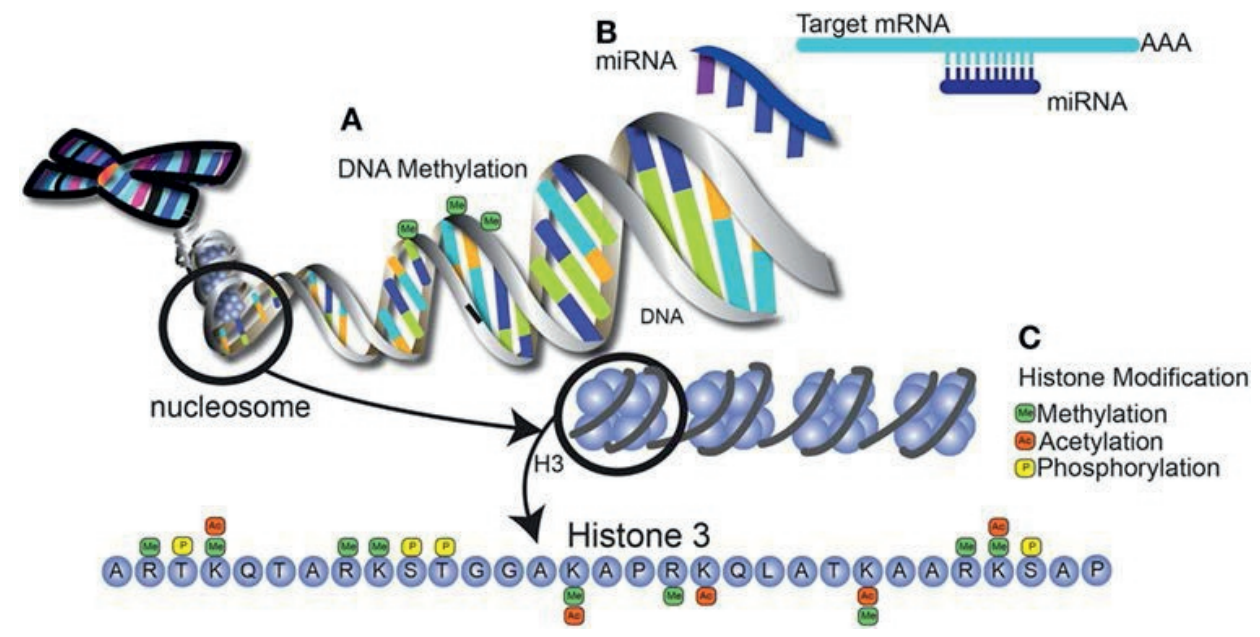

Figure 1. Major epigenetic modifications altering gene expression via multiple mechanisms (adapted from Fonseca et al., 2018).

Several triggers that promote transcription of pro-inflammatory cytokines (Tratter and Archer, 2007) can maintain chronic inflammation, which is the hallmark of hyperoxic lung injury. Transcription factors target gene transcription after activation and recruit transcriptional co-activators and chromatin-remodeling enzymes that allow subsequent inflammatory gene expression (Tratter and Archer, 2007). Therefore, inflammatory lung diseases were reported to be characterized by increased expression of several inflammatory genes that have been regulated by pro-inflammatory transcription factors (Adcock, 2005). Changes in histone acetylation are involved in induction of proinflammatory genes in human lung cells. Histone acetyltransferases (HATs) are expressed and activated in an abnormal fashion in inflammatory diseases and, histone deacetylase (HDAC) inhibitors show anti-inflammatory effects (Barnes et al.,2005). As chronic inflammation alters HAT/HDAC ratio and results in histone hypoacetylation, HDAC inhibitors were suggested to change the acetylation status of histones in order to prevent these changes (Ito et al., 2007).

Although this association between epigenetic mechanism and inflammation was well established in the pathogenesis of some adult inflammatory lung diseases including asthma and chronic obstructive lung disease, there is very limited data about this topic in neonatal diseases (Barnes, 2009; Barnes et al., 2005; Ito et al., 2007). The first clinical study to evaluate the role of molecular signatures in development of BPD in ELBW infants was designed by Cohen et al. (Cohen et al., 2007). This study showed that infants who subsequently developed BPD had distinct signatures involving chromatin remodeling and histone acetylation in umbilical cord samples of infants. The authors concluded that newer therapeutic modalities might be developed for BPD as chromatin-remodeling pathways appeared to be differentially regulated in umbilical cord tissues of the subsequently BPD-developed preterm infants (Cohen et al., 2007). Therefore, the idea of 
using HDAC inhibitors for prevention of BPD development was suggested as a potential therapy by some authors in the presence of studies showing the role of epigenetic mechanisms, especially histone acetylation/deacetylation changes in BPD development (Cohen et al., 2007; Merritt et al., 2009). In addition, reduced lung and brain injury was reported in preterm lambs treated with HDAC inhibitors during mechanical ventilation. In this experimental study, histone deacetylation inhibition significantly improved alveolar formation in the lung associated with changes in specific histone modifications. Herein, mechanical ventilation was suggested to cause epigenetic alterations that can be reversed pharmacologically with therapeutic benefit (Hamvas et al., 2014). In the presence of all these data suggesting the possible role of epigenetic mechanisms in development of neonatal lung disease, we evaluated the effect of a HDAC inhibitor for prevention of BPD in a rodent model. Valproic acid (VPA) is a class I and II HDAC inhibitor that is clinically used as an anti- epileptic and mood-stabilizing agent. VPA has also antiinflammatory properties (Kim et al., 2007). Therefore, we tested the hypothesis that epigenetic mechanisms including histone acetylation-deacetylation process might play an important role in development of BPD. To this end, we used a neonatal rat model of hyperoxic lung injury in which we evaluated the efficacy of valproic acid as an HDAC inhibitor. VPA treatment had an effect on many aspects of inflammation but also significantly improved weight gain.

The effects on the lung were improvement of histopathologic grade and radial alveolar count and the expression of lamellar body membrane protein, while it decreased the number of TUNEL $(+)$ cells and active Caspase-3 expression in the rat pups exposed to hyperoxia. Expressions of TGF 33 and phospho-SMAD2 proteins and levels of tissue proinflammatory cytokines as well as lipid peroxidation biomarkers were reduced, while anti-oxidative enzyme activities were enhanced by VPA treatment. VPA administration also reduced HDAC activity while increasing acetylated $\mathrm{H} 3$ and $\mathrm{H} 4$ protein expressions. VPA treatment improved alveolarization, reduced fibrosis and inflammation, enhanced antioxidant activity, all of which suggested the inhibitory action of VPA on HDAC activity. In conclusion, we reported that VPA treatment might be a new therapeutic strategy for prevention of BPD by ameliorating lung damage through HDAC inhibition (Cetinkaya et al., 2015). Although VPA has been used for treatment of refractory neonatal convulsions, it may be associated with cognitive deficits, progressive hyperammonemia, cerebral edema and diminished level of consciousness (Unal et al., 2007). Therefore, more experimental and clinical data about the safety of VPA treatment are required before the clinical usage in neonates. As a result, we suggest that more experimental and clinical studies evaluating the role of epigenetic mechanisms and newer therapeutic agents acting on these pathways are required to support our findings. 


\section{CDP-choline for prevention of BPD}

Another drug-based approach to prevent BPD might be to influence the composition of the alveolar membrane. Cytidine 5'-diphosphocholine (CDP-choline), composed of cytidine and choline linked by a diphosphate bridge, is an endogenous intermediate in the synthesis of the major membrane phospholipid, phosphatidylcholine (PC). PC constitutes the most important phospholipid, accounting for $70-85 \%$ of the total surfactant phospholipids (Adibhatla et al., 2005; Zimmermann et al., 2005). In preterm infants with evolving BPD, some persisting surfactant abnormalities including an ongoing quantitative deficiency in surfactant components, decreased function of endogenous surfactant, and increased surfactant turnover were established. Therefore, to investigate the effects of surfactant replacement on BPD development, surfactant was administered in preterm infants still on mechanical ventilation for $>1$ weeks of life. As a result, decrease in $\mathrm{FiO}_{2}$ requirement, transient improvement in oxygenation, and increase in survival without BPD were all reported (Laughon et al., 2009; Merrill et al., 2011). However, no study evaluated the role of CDP-choline for prevention of BPD as primary endpoint. We therefore tested the hypothesis that exogenous administration of CDP-choline, an endogenous intermediate in the synthesis of the major surfactant component phosphatidylcholine, might be protective against hyperoxic lung injury in a neonatal rat model. In this experimental study, CDP-choline treatment significantly reduced hyperoxia-induced severe lung damage. Radial alveolar count and expression of lamellar body membrane protein were significantly recovered, and the number of apoptotic cells, active caspase-3 expression, and tissue proinflammatory cytokine levels were decreased by CDP-choline administration. Lung tissue and BAL phospholipid contents also showed significant increases after CDP-choline therapy. Therefore, this study suggested for the first time that CDP-choline should be a novel therapeutic option for the prevention of BPD (Cetinkaya et al., 2013). This drug may be easily tested in clinical trials since it was previously clinically used for treatment of RDS in preterm infants (Colombo et al., 1976; Valls i Soler et al., 1988).

As stated above, we suggest that newer therapies acting on different stages and mechanisms of BPD development, such as VPA and CDP-choline may be promising for future. However, more experimental and especially clinical studies are required to support our findings and facilitate the transmission of our experimental data on clinical usage.

\section{New strategies for prevention of NEC}

NEC is the most frequent and devastating gastrointestinal disease in preterm infants. It has a complex and multifactorial pathogenesis including intestinal immaturity associated with prematurity, formula feeding, abnormal intestinal microbial colonization, hypoxia- 
ischemia in the gut, and an excessive inflammatory response (Cassir et al., 2016). NEC was suggested to develop as a consequence of intestinal hyper-responsiveness to microbial ligands that initiates a cascade of inflammatory pathway (Hodzic et al., 2017). The intestinal epithelium maintains barrier integrity and provides a defense layer against pathogens (Ramanan and Cadwell, 2016). PC is the major lipid of GI mucus layer that establishes a protective surface and plays a key role in mucosal defense. Because defective PC layers were suggested to contribute to the development of inflammation and because NEC patients show significant disruption in the intestinal epithelium, we evaluated the cytoprotective effect of CDP-choline for treatment of NEC in a rat model (Cetinkaya et al., 2013; Hodzic et al., 2017; Stremmel et al., 2010). In this experimental study, CDP-choline administered for 3 days significantly ameliorated intestinal injury, decreased inflammation and apoptosis, and increased intestinal tissue phospholipid levels. It also decreased lipid peroxidation and improved antioxidant status. This study showed the important role of phospholipids and PC in the maintenance and protection of gastrointestinal mucosa in NEC (Cetinkaya et al., 2013). We therefore suggested, for the first time, that CDP-choline is preventive against NEC development and might be offered as a novel therapeutic option for prevention of NEC through amelioration of disruption in GI mucosa and epithelium.

\section{The combination of anti-oxidant and anti-inflammatory treatment in necrotizing enterocolitis}

Excessive inflammation is an important factor in the pathogenesis of NEC and animal studies established the role of several inflammatory mediators and oxidative stress resulting with the subsequent apoptosis and tissue necrosis (De Plaen, 2013). The reactive oxygen species and free radicals were also suggested to result with disruption of gut barrier as an important factor in the multifactorial pathogenesis. In addition, it is well known that premature infants have immature antioxidant defense systems (Marseglia et al., 2015; Perrone et al, 2014). Therefore, the efficacy of potential anti-inflammatory therapies was evaluated for limiting this inflammatory cascade in NEC. To date, only breast milk and probiotics were suggested to have potential anti-inflammatory properties for prevention of NEC (Gupta and Paria, 2016). As mentioned above, CDP-choline showed preventive effects by decreasing inflammation in a neonatal NEC model (Cetinkaya et al, 2013). All these data suggested that anti-inflammatory interventions might be beneficial for the prevention of NEC. However, as there is no single effective treatment strategy against NEC, newer therapeutic options are required to decrease the incidence of this multifactorial disease. Because combination therapies that interfere with different stages of pathogenesis showed beneficial effects in neonatal morbidities including HIE, we tested in a neonatal experimental NEC model the hypothesis that the combination of an anti-oxidant, cytoprotective agent with an anti-inflammatory one might be protective 
(Cekmez et al, 2013). In this research, we evaluated the effect of melatonin and prostaglandin E1 combination for the prevention of NEC. Melatonin is an effective potent antioxidant and free radical scavenger. It also has the capability to modulate inflammation. Misoprostol, a prostaglandin E1 analog, has a cytoprotective effect on intestinal mucosa (Davies et al., 2001). In addition, it also reduces inflammation and tissue injury. Therefore, we investigated the efficacy of either single and combination therapies of melatonin and misoprostol in a neonatal rat NEC model. The combination therapy exerted better protective effect compared with single administration of these agents. This was the first study that showed the preventive effect of combination therapy acting on different stages of NEC. Hence, this study may lead to evaluation of other combination therapies for prevention and/or treatment of NEC in future.

\section{Therapies targeting epigenetic mechanisms in hypoxic-ischemic encephalopathy}

Perinatal hypoxic-ischemic ( $\mathrm{HI}$ ) brain injury is an important cause of mortality and adverse neurological outcomes in term and near term infants. Fetal ischemia and hypoxia lead to activation of ion channels, glutamate release, activation of several enzymes, formation of free radicals, inflammation, and mitochondrial damage resulting with necrosis and apoptosis (McLean and Ferriero, 2004). During this HI brain injury cascade, a therapeutic window has been defined that range until 6 hours of life where neuroprotective agents can confer benefit to reduce either mortality or adverse neurological consequences. Although, the only established therapy for neuroprotection in term and near term infants is hypothermia, approximately $50 \%$ of affected infants still die or suffer from long-term neurological adverse outcomes (Jacobs et al., 2013). Therefore, newer strategies are required to decline the adverse outcomes associated with $\mathrm{HI}$ brain injury. However, it is important to elucidate the potentially harmful molecular pathways and newer neuroprotective agents that have capability to inhibit these destructive mechanisms (Bel and Groenendaal, 2016). For this purpose, we aimed to evaluate the possible epigenetic effect of uridine in a neonatal $\mathrm{HI}$ brain injury model in rats. Uridine, the principal circulating pyrimidine nucleoside and precursor of brain membrane phospholipids, was shown to decrease $\mathrm{HI}$ brain injury dose-dependently in a neonatal rat model by our group (Cansev et al., 2013). We therefore aimed to investigate the possible epigenetic regulation effect of uridine on histone acetylation/ deacetylation balance in neonatal $\mathrm{HI}$ brain injury as HDAC inhibitors were shown to reduce oxidation, inflammation and apoptosis (Wang et al., 2012). The neonatal HI brain injury model was used in this experimental setting. After $\mathrm{HI}$ insult, postnatal D7 rat pups were given uridine for 3 consecutive days intraperitoneally. Uridine treatment reduced brain infarct volume and apoptosis. In addition, uridine decreased HDAC activity and increased the expression of acetylated histone-3 and -4 protein levels (Koyuncuoglu et al., 2015). To the best of our 
knowledge, this was the first study that reported the HDAC inhibition mechanism of uridine in $\mathrm{HI}$ brain injury that was also associated with reduction of brain infarct and apoptosis. As a result, we suggest that HDAC inhibition may be important for prevention and/or treatment of neonatal $\mathrm{HI}$ brain injury and that neuroprotective agents which interfere with this cascade may confer benefit in the treatment of $\mathrm{HI}$ brain insult in future.

\section{Combination of neuroprotective therapies for HIE}

As mentioned above, although therapeutic hypothermia reduces the risk of death and major neurodevelopmental disability, it neither does provide complete neuroprotection nor stimulates the repair necessary for normal neurodevelopmental outcome (Cilio and Ferriero, 2010). The cellular mechanisms including excitotoxicity, free radical toxicity, lipid peroxidation, and inflammation resulting with neuronal cell death were suggested to be prevented by combination therapies acting on the different levels of this complex cascade (Juul and Ferriero, 2014; Peters and van Bel, 2001). To evaluate the combination therapy in HIE, two agents acting on different stages of $\mathrm{HI}$ brain injury were used in a modified Levine-Rice model. It was tested whether the early stage of HIE could be prevented by means of combining magnesium sulfate $\left(\mathrm{MgSO}_{4}\right)$, an $\mathrm{N}$-methyl-D- aspartate (NMDA) receptor antagonist acting on the early stage of excitotoxicity with melatonin as an indirect antioxidant and potent free radical scavenger that acts on the later stage of $\mathrm{HI}$ injury (Cetinkaya et al., 2011). In this experimental study, $\mathrm{MgSO}_{4}$ and melatonin, having different actions on the $\mathrm{HI}$ brain injury cascade, significantly reduced the infarcted brain volume and apoptosis, either alone or in combination. However, we could not show any additive benefit of this combination therapy (Cetinkaya et al., 2011). Following this study, we tested the neuroprotective effect of topiramate, an antiepileptic and AMPA/kainate receptor antagonist in $\mathrm{HI}$ brain injury. We evaluated its efficacy either alone or in combination with melatonin. We again hypothesized that combination of two neuroprotective agents acting on different stages of HIE might confer additional therapy.

Herein, topiramate and melatonin either alone or in combination, significantly reduced the size of infarcted area and apoptosis. However, the combination therapy did not show superiority to the single therapies (Ozyener et al., 2012). In recent years, in accordance with our hypothesis and results, most authors have suggested using combination therapy options that act on multiple mechanistic processes (Dixon et al., 2015; Zalewska et al., 2015). For this purpose, it may be reasonable to suggest combining a neuroprotective agent with hypothermia or combining two neuroprotective agents that are known to act on different phases of $\mathrm{HI}$ injury to achieve better neuroprotection in infants with severe HIE (Dixon et al., 2015; Zalewska et al., 2015). Therefore, future studies are warranted investigating the best synergistic combination therapy for effective treatment of HIE. 


\section{The importance of experimental studies in neonatology}

Since performing clinical studies in neonates are extremely challenging due to ethical issues and risks associated with unproven therapies, performing well-planned basic experiments on animal models is essential beforehand. Experimental data obtained from animal models can be translated into clinical practice with the goal of establishing novel treatment options in sophisticated human studies. However, we must keep in mind that a large number of potential therapies that were effective in animals were not proven effective in subsequent clinical trials. Therefore, it is important to perform well-designed experimental models that closely mimic the human diseases. We suggest that translational research from the experimental animal laboratory to the human clinic is essential to improve neonatal outcomes for both prevention and treatment of several morbidities with multifactorial pathogenesis and limited therapeutic options. The main advantages of our experimental studies include the possibility of usage of these treatments clinically in neonates, thereby translating results from bench to bedside.

\section{Conclusion and future perspectives}

Advances in neonatology resulted in increased survival rates of infants. In parallel, BPD, NEC and adverse neurological outcomes among ELBW infants as well as perinatal asphyxia in term infants remain a significant problem. Therefore, the aim for the future is to decrease rates of these morbidities in both term and preterm survivors.

In view of the currently available data, we know that there is no single effective therapy for neither BPD, nor NEC or HIE. We therefore need alternative and more efficacious therapies for treatment as well as prevention of these morbidities. Based on our experimental studies, we suggest a potential role for CDP-choline in the prevention of both BPD and NEC. As the injury in the lung and gastrointestinal system usually disrupts the epithelium and show a distribution from the injured epithelium, we evaluated the preventive and therapeutic efficacy of CDP-choline as an agent to improve the injured epithelial and mucosal layer in the developmental process of BPD and NEC. Our studies were the first in the literature that reported the beneficial effects of CDP-choline on the maintenance of normal membrane structure and prevention of inflammation and lipid peroxidation, the hallmarks of BPD and NEC. As the importance of genetic and epigenetic mechanisms were identified in the development of neonatal morbidities in recent years, therapeutic approaches targeting epigenetic mechanisms should be considered in prevention of BPD and HIE. Valproic acid and uridine treatments reduced HDAC activity and increased the expression of acetylated histone proteins and were therefore found to be effective for prevention and treatment of BPD and HIE in our studies. Although the HDAC activity of valproic acid was well known, our group showed the HDAC activity of uridine in neonatal $\mathrm{HI}$ brain injury for the first time. These were the first studies in the 
literature that reported the beneficial effects of therapeutic agents acting on epigenetic mechanisms for treatment and/or prevention of BPD and HIE. The agents acting on the similar pathways with the same pathogenesis may confer benefits in the prevention and/or treatment of different neonatal diseases such as HIE, BPD and NEC. Although the combination therapy with magnesium sulfate, topiramate, and melatonin was found to be neuroprotective in experimental $\mathrm{HI}$ brain injury models, we still need to find the best combination therapy, possibly in conjunction with hypothermia and appropriate timing. Therefore, greater number of experimental studies are required to establish novel and effective therapies for prevention and/or treatment of conditions like BPD, NEC and HIE before going into clinical trials.

We believe that investigating therapeutic potential of easily accessible and non-toxic agents that target and modulate pathogenic mechanisms of neonatal diseases, alone or in combination, in well-established animal models will certainly provide extensive information on the extent and quality of the treatment strategy that is tested. Data derived from these studies must further be utilized in clinical studies in order to translate the knowledge from bench to bedside and help to reduce neonatal mortality and morbidity by testing new therapeutic strategies against BPD, NEC and HIE. It may be speculated that the success of translational research in neonates leading to discovery of novel beneficial therapeutic approaches will be related with the quality of well-designed and universally accepted experimental models as well as with the choice of right agents. 


\section{References}

1. Adcock, I.M., Ito, K., and Barnes, P.J. (2005). Histone deacetylation: an important mechanism in inflammatory lung diseases. CPOD 2: 445-455.

2. Adibhatla, R.M., and Hatcher, J.F. (2005). Cytidine 5'-diphosphocholine (CDP-choline) in stroke and other CNS disorders. Neurochemical research 30: 15-23.

3. Barnes, P.J. (2009). Targeting the epigenome in the treatment of asthma and chronic obstructive pulmonary disease. Proceedings of the American Thoracic Society 6: 693-696.

4. Barnes, P.J., Adcock, I.M., and Ito, K. (2005). Histone acetylation and deacetylation: importance in inflammatory lung diseases. European respiratory journal 25: 552-563.

5. Bel, F.V., and Groenendaal, F. (2016). Drugs for neuroprotection after birth asphyxia: pharmacologic adjuncts to hypothermia. Seminars in perinatology 40: 152-159.

6. Cansev, M., Minbay, Z., Goren, B., Yaylagul, E.O., Cetinkaya, M., Koksal, N., and Alkan T. (2013). Neuroprotective effects of uridine in a rat model of neonatal hypoxic-ischemic encephalopathy. Neuroscience letters 542: 65-70.

7. Cassir, N., Simeoni, U., and La Scola, B. (2016). Gut microbiota and pathogenesis of necrotizing enterocolitis in preterm neonates. Future microbiology 11: 273-292.

8. Cekmez, F., Cetinkaya, M., Tayman, C., Canpolat, F.E., Kafa, I.M., Uysal, S., Tunc, T., and Sarıc S.U. (2013). Evaluation of melatonin and prostaglandin E1 combination on necrotizing enterocolitis model in neonatal rats. Regulatory peptides 184: 121-125.

9. Cetinkaya, M., Alkan, T., Ozyener, F., Kafa, I.M., Kurt, M.A., and Koksal N. (2011). Possible neuroprotective effects of magnesium sulphate and melatonin as both pre- and post- treatment in a neonatal hypoxicischemic rat model. Neonatology 99: 302-310.

10. Cetinkaya, M., Cansev, M., Cekmez, F., Tayman, C., Canpolat, F.E., Kafa, I.M., Uysal, S., Tunc, T., and SarıCI S.U. (2013). CDP-choline reduces severity of intestinal injury in a neonatal rat model of necrotizing enterocolitis. Journal of surgical research 183: 119-128.

11. Cetinkaya, M., Cansev, M., Cekmez, F., Tayman, C., Canpolat, F.E., Kafa, I.M., Yaylagul, E.O., Kramer, B.W., and Sarici S.U. (2015). Protective effects of valproic acid, a histone deacetylase inhibitor, against hyperoxic lung injury in a neonatal rat model. PLoS One 10(5): e0126028.

12. Cetinkaya, M., Cansev, M., Kafa, I.M., Tayman, C., Cekmez, F., Canpolat, F.E., Tunc, T., and Sarıcı, S.U. (2013). Cytidine 5'-diphosphocholine ameliorates hyperoxic lung injury in a neonatal rat model. Pediatric research 74: 26-33.

13. Cilio, M.R., and Ferriero, D.M. (2010). Synergistic neuroprotective therapies with hypothermia. Seminars in fetal \& neonatal medicine 15: 293-298.

14. Cohen, J., Van Marter, L.J., Sun, Y., Allred, E., Leviton, A., and Kohane, I.S. (2007). Perturbation of altered gene expression of the chromatin remodeling pathway in premature infants at risk for bronchopulmonary dysplasia. Genome biology 8: R210.

15. Colombo, M.L., Dogliani, P., and Raggi, M. (1976). Relation between citicoline and pulmonary surfactant. Recent acquisitions in the treatment of respiratory syndromes of the newborn. Minerva pediatrics 28 : 2303-2310.

16. Davies, N.M., Longstreth, J., and Jamali, F. (2001). Misoprostol therapeutics revisited. Pharmacotherapy 21: 60-73.

17. De Plaen, I.G. (2013). Inflammatory signaling in necrotizing enterocolitis. Clinics in perinatology 40: 109124.

18. Dixon, B.J., Reis, C., Ho, W.M., Tang, J., and Zhang, J.H. (2015). Neuroprotective strategies after neonatal hypoxic ischemic encephalopathy. International journal of molecular sciences 16: 22368-22401.

19. Fonseca, W., Lukacs, N.W., and Ptaschinski, C. (2018). Factors affecting the immunity to respiratory syncytial virus: from epigenetics to microbiome. Frontiers in immunology 9: 226.

20. Gantert, M., Been, J.V., Gavilanes, A.W.D., Garnier, Y., Zimmermann, L.J., and Kramer, B.W. (2010). Chorioamnionitis: a multiorgan disease of the fetus? Journal of perinatology 30: S21- S30. 
21. Gupta, A., and Paria, A. (2016). Aetiology and medical management of NEC. Early human development 97 : 17-23.

22. Hamvas, A., Deterding, R., Balch, W.E., Schwartz, D.A., Albertine, K.H., Whitsett, J.A., Cardoso, W.V., Kotton, D.N., Kourembanas, S., and Hagood J.S. (2014). Diffuse lung disease in children: summary of a scientific conference. Pediatric pulmonology 49: 400-409.

23. Hodzic, Z., Bolock, A.M., and Good, M. (2017). The role of mucosal immunity in the pathogenesis of necrotizing enterocolitis. Frontiers in pediatrics 5: 40.

24. Ito, K., Charron, C.E., and Adcock, I.M. (2007). Impact of protein acetylation in inflammatory lung diseases. Pharmacology \& therapeutics 116: 249-265.

25. Jacobs, S.E., Berg, M., Hunt, R., Tarrow-Mordi, W.O., Inder, T.E., and Davis, P.G. (2013). Cooling for newborns with hypoxic ischaemic encephalopathy. Cochrane database of systematic reviews 1: CD003311.

26. Jensen, E.A., Foglia, E.E., and Schmidt, B. (2015). Evidence-based pharmacologic therapies for prevention of bronchopulmonary dysplasia: application of the grading of recommendations assessment, development, and evaluation methodology. Clinics in perinatology 42: 755-759.

27. Juul, S.E., and Ferriero, D.M. (2014). Pharmacological neuroprotective strategies in neonatal brain injury. Clinics in perinatology 41: 119-131.

28. Kim, H.J., Rowe, M., Ren, M., Hong, J.S., Chen, P.S., and Chuang DM. (2007). Histone deacetylase inhibitors exhibit anti-inflammatory and neuroprotective effects in a rat permanent ischemic model of stroke: multiple mechanisms of action. Journal of pharmacology and experimental therapeutics 321: 892-901.

29. Koyuncuoglu, T., Turkyilmaz, M., Goren, B., Cetinkaya, M., Cansev, M., and Alkan, T. (2015). Uridine protects against hypoxic-ischemic brain injury by reducing histone decetylase activity in neonatal rats. Restorative neurology and neuroscience 33: 777-784.

30. Laughon, M., Bose, C., Moya, F., Aschner, J., Dunn, S.M., Morabito, C., Cummings J.J., Segal, R., Guardia, C., Liu, G., and Surfaxin Study Group. (2009). A pilot randomized, controlled trial of later treatment with a peptide-containing, synthetic surfactant for the prevention of bronchopulmonary dysplasia. Pediatrics 123 : 89-96.

31. Li, B., Carey, M., and Workman, J.L. (2007). The role of chromatin during transcription. Cell 128: 707-719.

32. Marseglia, L., D’Angelo, G., Manti, S., Aversa, S., Reiter, R.J., Antonuccio, P., Centorrino, A., Romeo, C., Impellizerri, P., and Gitto, E. (2015). Oxidative-stress mediated damage in newborns with necrotizing enterocolitis: a possible role of melatonin. American journal of perinatology 32: 905-909.

33. McLean, C., and Ferriero, G. (2004). Mechanisms of hypoxic-ischemic brain injury in the term infant. Seminars in perinatology 28: 425-432.

34. Merrill, J.D., Ballard, P.L., Courtney, S.E., Durand, D.J., Hamvas, A., Hibbs, A.M., Lu, K.W., Ryan, R.M., Reynolds, A.M., Spence, K., Steinhorn, R.H., Truog, W.E., Eichenwald, E.C., and Ballard, R.A. (2011). Pilot trial of late booster doses of surfactant for ventilated premature infants. Journal of perinatology 31: 599606.

35. Merritt, T.A., Deming, T.T., Boynton, B.R. (2009). The new bronchopulmonary dysplasia: challenges and commentary. Seminars in fetal \& neonatal medicine 14: 345-357.

36. Merritt, T.A., Gadzınowski, J., Mazela, J., and Adamczak, A.M. (2002). Epigenetic influences in the development of bronchopulmonary dysplasia. Archieves of perinatal medicine 17: 17-22.

37. Ozyener, F., Cetinkaya, M., Alkan, T., Goren, B., Kafa, I.M., Kurt, M.A., and Koksal, N. (2012). Neuroprotective effects of melatonin administered alone or in combination with topiramate in neonatal hypoxic-ischemic rat model. Restorative neurology and neuroscience 30: 435-444.

38. Peeters, C., and van Bel, F. (2001). Pharmacotherapeutical of post-hypoxic-ischemic brain injury in the newborn. Biology of the neonate 79: 274-280.

39. Perrone, S., Tataranno, M.L., Santacroce, A., Negro, S., and Buonocore, G. (2014). The role of oxidative stress on necrotizing enterocolitis in very low birth weight infants. Current pediatric reviews 10: 202-207.

40. Poets, C.F., and Lorenz, L. (2018). Prevention of bronchopulmonary dysplasia in extremely low gestational age neonates: current evidence. Arch Dis Child Fetal Neonatal Med 103: F285-F291.

41. Ramanman, D., and Cadwell, K. (2016). Intrinsic defense mechanisms of the intestinal epithelium. Cell host microbe 19: 434-441. 
42. Stoll, B.J., Hansen, N.I., Bell, E.F., Walsh, M.C., Carlo, W.A., Shankaran, S., Laptook, A.R.,Sánchez, P.J., Van Meurs, K.P., Wyckoff, M., Das, A., Hale, E.C., Ball, M.B., Newman, N.S., Schibler, K., Poindexter, B.B., Kennedy, K.A., Cotten, C.M., Watterberg, K.L., D’Angio, C.T., Demauro S.B., Truog, W.E., Devaskar, U., Higgins, R.D., and Eunice Kennedy Shriver National Institute of Child Health and Human Development Neonatal Research Network. (2015). Trends in care practices, morbidity and mortality of extremely preterm neonates, 1993- 2012. JAMA 314: 1039-1051.

43. Stremmel, W., Hanemann, A., Ehehalt, R., Karner, M., and Braun, A. (2010) Phosphotydilcholine and the mucus layer: evidence of therapeutic efficacy in ulcerative colitis. Digestive diseases 28: 490-496.

44. Tratter, K.W., and Archer, T.K. (2007). Nuclear receptors and chromatin remodeling machinery. Molecular and cellular endocrinology 265-266: 162-167.

45. Unal, E., Kaya, U., and Aydın, K. (2007). Fatal valproate overdose in a newborn baby. Human \& experimental toxicology 26:453-456.

46. Valls i Soler, A., Sanjurjo, P., and Vazquez Cordero, C. (1988). Controlled study of the administration of CDPcholine to preterm newborn infants with respiratory distress syndrome. Anales espanoles de pediatria 28: 493-496

47. Wang, B., Zhu, X., Kim, Y., Li, J., Huang, S., Saleem, S., Li, R.C., Xu, Y., Dore, S., Cao, W. (2012). Histone acetylation inhibition activates transcription factor $\mathrm{Nrf2}$ and protects against cerebral ishemic damage. Free radical biology and medicine 52: 928-936.

48. Zalewska, T., Jaworska, J., and Ziemka-Nalecz, M. (2015). Current and experimental pharmacological approaches in neonatal hypoxic- ischemic encephalopathy. Current pharmaceutical design 21: 1433-1439.

49. Zimmermann, L.J., Janssen, D.J., Tibboel, D., Hamvas, A., and Carnielli, V.P. (2005). Surfactant metabolism in the neonate. Biology of the neonate 87: 296-307. 

Chapter

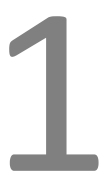

Valorization 

This valorization chapter describes how the outcomes of this thesis contribute to neonatal units, parents, society as well as research and industry. The results described in this thesis enable new treatment and prevention modalities in both term and premature infants.

\section{Societal Importance}

Preterm birth is a very important public health priority worldwide; approximately 15 million babies are born preterm every year and preterm birth complications are the leading cause of death among children under 5 years of age (Liu et al., 2016). In addition, preterm birth is also associated with increased morbidity as well as with increased health care costs because of longer hospital stays and a greater need for intensive care due to medical care related to morbidities of prematurity. In a study from the US, the disproportionate share of neonatal care was highlighted (Barradas et al., 2016). Although 9.1\% of all hospitalizations were associated with preterm birth, these hospitalizations accounted for $43.4 \%$ of total costs. In addition, $5.9 \%$ of the same cohort required rehospitalization, the costs of which accounted for $22.6 \%$ of all costs. Therefore, we need evidence-based strategies to prevent prematurity and prematurity-related morbidities. As more immature infants survive due to the advances in neonatal care, the incidences of bronchopulmonary dysplasia (BPD) and necrotizing enterocolitis (NEC) do not decrease (Stoll et al., 2015). Hence, it is reasonable and also cost-effective to develop targeted therapies against morbidities such as BPD and NEC.

In addition to prematurity, neonatal hypoxic ischemic encephalopathy (HIE) presents also a significant clinical burden with its persistent high mortality and morbidity rates. Worldwide, four million newborn infants experience birth asphyxia each year, accounting for an estimated one million deaths and 42 million disability-adjusted life years (Nair, J., and Kumar, V.H.S., 2018). Although therapeutic hypothermia has been accepted as the standard of care for infants with moderate to severe HIE, it has not definitively changed overall outcomes in severe HIE. HIE has a multifactorial pathogenesis and multiple cellular events including excitotoxicity, inflammation and oxidative stress lead to cellular damage. Due to this complex events cascade, combination therapies acting on different stages of hypoxic ischemic injury were suggested as a novel approach to reach different targets in the setting of HIE. These therapy targets in HIE include prevention of acute lesions, increase in size of the therapeutic time window for protection, and enhanced repair in the long term (Cilio, M.R. and Ferriero, D.M., 2010). Therefore, development of alternative therapies, especially the combined use of neuroprotective agents, were suggested to provide more benefit for prevention of HIE-related devastating neurological disability (Douglas-Escobar, M., and Weiss M.D., 2015).

Since prematurity, prematurity-related morbidities and HIE contribute to a significantly higher burden of neonatal mortality and morbidity globally, there is a need 
for alternative and supplementary therapeutic agents. In order to have global applicability, therapies need to have low costs and should be easily and readily available (Costa et al., 2018). However, research involving critically ill neonates is very hard to perform due to some unique ethical concerns. The design of trials, the informed consent process, and the implementation of studies in critically ill neonates is very difficult because these subjects are vulnerable and critically ill, have a significant risk of dying or a poor prognosis when surviving. Despite these ethical concerns, it is very crucial to perform research trials for introduction of new drugs, devices, and interventions and to develop the necessary evidence of efficacy and toxicity to support the use of these medications (Fleischman, A.R., 2016). It is also important to establish the safety and efficacy of such drugs in preterm infants, especially in the hospitalized population with lower birth weight and gestational age who are actually on multiple drugs (Costa et al., 2018). The drug-drug interactions in these patients are not well studied and may be further complicated by prematurity as such. From the ethical side, the main problem is to increase the effectiveness and to improve short- and long-term effects of these promising modalities in preterm infants while conducting drug studies in neonates within the ethical framework of respect for persons, justice, and beneficence (Ward, R.M., and Sherwin, R.C., 2015). To bypass these ethical problems related to clinical studies, we can perform experimental studies in the preclinical stage, the results of which can be translated into clinical usage. The experimental studies for prevention and treatment of important prematurity-associated neonatal morbidities and HIE that were reported in this thesis showed promising results for the possible therapeutic usage of some agents such as valproic acid, CDP-choline, melatonin, prostaglandin E1, uridine and topiramate. After those encouraging results from our experimental studies, the efficacy and safety of these therapies should be evaluated in this vulnerable group with further clinical studies. In accordance with this idea, limited number of clinical studies that evaluated the use of melatonin in HIE and neonatal sepsis in newborns have been published (Aly et al., 2015 and El Frargy, et al. 2015). Similarly, the safety and efficacy of topiramate was also evaluated in a recent feasibility study (Filippi et al., 2018). Therefore, I suggest that these preliminary studies may work as a pioneer for planning further larger clinical studies that may result with translation of our experimental results to clinical usage for these agents.

\section{Contributions of this thesis to understanding of neonatal diseases and planning future studies for health professional societies}

The findings in this thesis are of importance and of additional value for current understanding and further research in the prevention of prematurity related morbidities and HIE in term babies. Prematurity-related morbidities including BPD and NEC have multifactorial pathogenesis. Therefore, there is no single curative treatment for these 
morbidities and new therapeutic agents are required to prevent and/or treat these morbidities.

Epigenetic mechanisms including DNA base modifications such as methylation, alteration of histones resulting in chromatin modification, as well as the actions of noncoding RNA are required for both lung modelling and remodeling. Recently, epigenetic regulation was also suggested to play an important role in development of chronic lung diseases including BPD (Merritt et al., 2011). In this thesis, valproic acid, which is a histone deacetylase inhibitor, was suggested as an alternative preventive treatment approach for neonatal hyperoxic lung injury in preterm infants due to its epigenetic effects. Since valproic acid was reported to be used as a therapeutic agent for treatment of severe neonatal seizures, its effectiveness against BPD in preterm infants should be evaluated in future clinical studies in the presence of the data provided by our preclinical study.

The complex multifactorial pathogenesis of NEC results in intestinal dysfunction, inflammation, injury and necrosis. All these events result in the disruption of cell membranes in all organ systems including lung and intestine. Therefore, restoration of cell membrane functions may provide benefits in these conditions. Herein, CDP-choline, an endogenous intermediate of major membrane phospholipids, was shown to reduce both hyperoxic lung injury and NEC by several different mechanisms of action. As CDPcholine was administered to preterm infants for treatment of respiratory distress syndrome, its usage for either prevention and/or treatment of both NEC and BPD in these infants may be evaluated by future clinical studies. As mentioned above, due to the complex pathogenesis of these morbidities, combinations of therapies instead of a single therapeutic agent should also be evaluated for both treatment and/or prevention of BPD and NEC in preterm infants. This combination therapeutic approach may also be tested for newer treatment strategies for HIE as mentioned in the thesis. Especially, combination of some inexpensive therapies such as magnesium sulfate and melatonin as mentioned in the thesis may enable neonatologists to provide an effective therapy in developing countries that lack therapeutic hypothermia. The results of this thesis will encourage clinical researchers to test therapeutic approaches best suited for the resources in their environment.

\section{Contributions of this thesis to health economics and hospital costs}

Preterm birth and prematurity represent a global economic problem due to longer hospitalization periods and re-hospitalizations after discharge. In addition, prematurityrelated increased neurological and pulmonary conditions such as cerebral palsy, asthma and learning difficulties may lead to an economic burden due to the increased health costs. Therefore, the newer and inexpensive therapeutic approaches and the combination of therapies described in this thesis may help to decrease the health care 
costs in these infants, which is important for both the family, and the society in a country. These experimental therapeutic approaches may offer new treatment strategies in the NICU perspective by either decreasing the duration of hospitalization or decreasing the incidence and/or severity of adverse outcomes associated with these morbidities. It may also reduce the need of re-hospitalization associated with the effects of abnormal lung injury or intestinal damage during hospitalization.

\section{Contributions of this thesis to parents}

Preterm birth has also been linked to increased parental stress, depression, and anxiety (Pace et al., 2016). Parental anxiety can be decreased by detailed discussion of expected prematurity-related issues and possible treatment strategies. Herein, the results of this thesis may offer understanding and hope for their infants to have a possible cure in the presence of risk factors for BPD or NEC development.

\section{Future perspectives}

This thesis provides a combination of several therapeutic approaches for the prevention of important neonatal morbidities in both term and preterm infants. The reported experimental studies justify clinical applications and translations. This translation must be done in carefully designed clinical trials, where not only the short-term benefit is tested, but also data on the long-term follow-up is collected. Therefore, the studies in this thesis serve both the pathophysiological understanding and the therapeutic innovation. All these improvements may guide clinicians to perform human studies with the idea in mind that it is more important to develop preventive strategies rather than treatment strategies for these morbidities. 


\section{References}

1. Aly, H., Elmahdy, H., El-Dib, M., Rowisha, M., Awny, M., El-Gohary, T., Elbatch, M., Hamisa, M., and ElMashad A.R. (2015) Melatonin use for neuroprotection in perinatal asphyxia: a randomized controlled pilot study. Journal of perinatology 35: 186-191.

2. Barradas, D.T., Wasserman, M.P., Daniel-Robinson, L., Bruce, M.A., Disantis, K.I., Navarro, F.H., Jones, W.A., Manzi, N.M., Smith, M.W., and Goodness, M. B. (2016). Hospital utilization and costs among preterm Infants by payer: nationwide inpatient sample, 2009. Maternal and child health journal 20: 808-828.

3. Cilio, M.R., and Ferriero, D.M. (2010). Synergistic neuroprotective therapies with hypothermia. Seminars in fetal \& neonatal medicine 15: 293-298.

4. Costa, H.T.M.L., Costa, T.X., Martins, R.R., and Oliveira, A.G. (2018). Use of off-label and unlicensed medicines in neonatal intensive care. PloSOne 13(9): e0204427.

5. Douglas-Escobar, M., and Weiss, M.D. (2015). Hypoxic-ischemic encephalopathy: a review for the clinician. JAMA pediatrics 169: 397-403.

6. El Frargy, M., El-Sharkawy, H.M., and Attia, G.F. (2015). Use of melatonin as an adjuvant therapy in neonatal sepsis. Journal of neonatal-perinatal medicine 8: 227-232.

7. Filippi, L., Fiorini, P., Catarzi, S., Berti, E., Padrini, L., Landucci, E., Donzelli, G., Bartalena, L., Fiorentini, E., Boldrini, A., Giampietri, M., Scaramuzzo, R.T., la Marca, G., Della Bona, M.L., Fiori, S., Tinelli, F., Bancale, A., Guzzetta, A., Cioni, G., Pisano, T., Falchi, M., and Guerrini, R. (2018). Safety and efficacy of topiramate in neonates with hypoxic ischemic encephalopathy treated with hypothermia (NeoNATI): a feasibility study. The journal of maternal- fetal \& neonatal medicine 31: 973-980.

8. Fleischman, A.R. (2016). Ethical issues in neonatal research involving human subjects. Seminars in perinatology 40: 247-253.

9. Liu, L., Oza, S., Hogan, L., Chu,Y., Perin, J., Zhu, J., Lawn, J.E., Cousens, S., Mathers, C., and Black, R.E. (2016). Global, regional, and national causes of under-5 mortality in 2000-15: an updated systematic analysis with implications for the Sustainable Development Goals. Lancet 388: 3027-3035.

10. Merritt, T.A., Gadzinowski, J., Mazela, J., and Adamczak, A.M. (2011). Epigenetic influences in the development of bronchopulmonary dysplasia. Archieves of perinatal medicine 17: 17-22.

11. Nair, J., and Kumar, V.H.S. (2018). Current and emerging therapies in the management of hypoxic ischemic encephalopathy in neonates. Children (Basel) 5: 99.

12. Pace, C.C., Spittle, A.J., Molesworth, C.M., Lee, K.J., Northam, E.A., Cheong, J.L., Davis, P.G., Doyle, L.W., Treyvaud, K., and Anderson, P.J. (2016). Evolution of depression and anxiety symptoms in parents of very preterm infants during the newborn period. JAMA pediatrics 170: 863-870.

13. Stoll, B.J., Hansen, N.I., Bell, E.F., Walsh, M.C., Carlo, W.A., Shankaran, S., Laptook, A.R.,Sánchez, P.J., Van Meurs, K.P., Wyckoff, M., Das, A., Hale, E.C., Ball, M.B., Newman, N.S., Schibler, K., Poindexter, B.B., Kennedy, K.A., Cotton, C.M., Watterberg, K.L., D'Angio, C.T., DeMauro, S.B., Truog, W.E., Devaskar, U., Higgins, R.D. and Eunice Kennedy Shriver National Institute of Child Health and Human Development Neonatal Research Network (2015). Trends in care practices, morbidity and mortality of extremely preterm neonates, 1993-2012. JAMA 314: 1039-1051.

14. Ward, R.M., and Sherwin, R.C. (2015). Ethics of drug studies in the newborn. Paediatric drugs 17: 37-42. 

Chapter

Curriculum vitae 



\section{Merih CETINKAYA, MD., PhD., \\ Professor of Pediatrics and Neonatology \\ Health Sciences University \\ Kanuni Sultan Suleyman Training and Research Hospital, \\ Department of Neonatology, Istanbul, TURKEY \\ +90- 212-4955920, +90 532 3678867, drmerih@yahoo.com}

Merih Cetinkaya graduated from Dokuz Eylul University, Faculty of Medicine in İzmir, Turkey. He completed his pediatric residency and neonatology fellowship in Uludag University, Faculty of Medicine. He also received his PhD from Physiology Department of Uludag University. He was in Vermont University, Fletcher Allen Health Care and Harvard University, Brigham and Women's Hospital as an observer in 2009.

He became Associate Professor of Pediatrics in 2011. In 2017, he became Professor of Pediatrics in Health Sciences University in Turkey. He currently works as chief of the Neonatology Department of the Kanuni Sultan Suleyman Training and Research Hospital in Istanbul that serves as a referral center in Neonatology and Perinatology. His primary research areas involve new therapies for prevention and treatment of neonatal hypoxiaischemia, hyperoxic lung injury, and necrotizing enterocolitis. He also works on neonatal outcomes of preeclampsia and new biomarkers in the diagnosis of neonatal sepsis and necrotizing enterocolitis. His PhD in Maastricht University, Department of Experimental Perinatology is a continuation of these subjects.

He published over 80 peer-reviewed manuscripts. He was the recipient of several national and international awards. He was awarded the ESPNIC Young Investigator Award at the 5th Congress of the European Academy of Pediatric Societies in October 2014. He also received the 1st SIN (Italian Neonatology Society) European Young Researcher Neonatology Award. He was the recipient of the Neonatology Investigator Award of the Turkish Neonatology Society in 2011 and 2016. He was also selected as a runner-up for the 4rd International Congress of the European Academy of Paediatric Societies Investigator Prize in 2012. He is a frequent speaker at both international and national congresses and meetings.

Merih Cetinkaya is also a member of the Editorial Board and reviewer of several national and international medical journals. He works as a co-investigator in several international clinical studies. His primary aim is to develop new strategies against the most common morbidities in pediatrics, especially in neonatology. 


\section{INTERNATIONAL AWARDS}

Cetinkaya, M., Varturk, I., Korachi, M., Guven, S., Akın, I.M., Erener-Ercan, T., and Buyukkale, G. (2014). Association of E-NOS Gene Polymorphism in Development of Bronchopulmonary Dysplasia. The 5th Congress of the European Academy of Paediatric Societies EAPS, ESPNIC Paediatric and Neonatal Intensive Care Young Investigator Award 2014, October 17-21, Barcelona, Spain.

Cetinkaya, M., Ozyener, F., Alkan, T., Kafa, I.M., Kurt, M.A., and Koksal, N. (2011). Possible neuroprotective effects of magnesium sulfate and melatonin as both pre- and posttreatment in a neonatal hypoxic-ischemic rat model, 1st SIN (Italian Neonatology Society) European Young Researcher Neonatology Award, 11-14 October, Sorrento, Italy.

\section{PEER-REVIEWED PUBLICATIONS}

Acar, D.B., Kavuncuoglu, S., Cetinkaya, M., Petmezci, E., Dursun, M., Korkmaz, O., and Altuncu, E.K. (2015). Assesment of the place of tubular reabsorption of phosphorus in the diagnosis of osteopenia of prematurity. Turk pediatri arsivi 50: 45-50.

Alan, S., Erdeve, O., Cakir, U., Akduman, H., Zenciroglu, A., Akcakus, M., Tunc, T., Gokmen, Z., Ates, C., Atasay, B., Arsan, S., and TurkNICU-RSV Trial Group. (2016). The journal of maternal-fetal \& neonatal medicine 29: 2186-2193.

Ali, R., Ozkalemkas, F., Kimya, Y., Koksal, N., Ozkan, H., Ozkocaman, V., Hoyrazlı, A., Cetinkaya, M., and Tunalı, A. (2009). Acute leukemia and pregnancy. Leukemia research 33: e26-28.

Babayigit, A., Cebeci, B., Buyukkale, G., Yilmaz, S.S., Bornaun, H., Oztarhan, K., Gokce, M., and Cetinkaya, M. (2015). Treatment of neonatal fungal infective endocarditis with recombinant tissue plasminogen activator in a low birth weight infant: case report and review of the literature. Mycoses 58: 578-581.

Babayigit, A., Ozaydın, S., Cetinkaya, M., and Sander, S. (2018). Neonatal gastric perforations in very low birth weight infants: a single center experience and review of the literature. Pediatric surgery international 34: 79-84.

Bas, A.Y., Koc, E., Dilmen, U., and ROP Neonatal Study Group. (2015). Incidence and severity of retinopathy of prematurity. British journal of ophtalmology 99: 1311-1314.

Bayram, F., Ozerkan, K., Cengiz, C., Develioglu, O., and Cetinkaya, M. (2010). Perinatal asphyxia is associated with the umblical cord nucleated red blood cell count in preeclamptic pregnancies. Journal of obstetrics and gynaecology 30: 383-386. 
Bornaun, H., Oztarhan, K., Erener-Ercan, T., Dedeoglu, R., Tugcu, D., Aydogmus, C., Cetinkaya, M., and Kavuncuoglu, S. (2016). Regression of cardiac rhabdomyomas in a neonate after Everolismus therapy. Case reports in pediatrics 2016: 8712962.

Bornaun, H., Yartası Tik, E., Keskindemirci, G., Ekiz, A., Oztarhan, K., Dedeoglu, R., and Cetinkaya, M. (2016). Right atrial appendage aneurysm in a newborn diagnosed with fetal echocardiography. Case reports in pediatrics 2016: 8616918.

Buyukkale, G., Cetinkaya, M., Akcay, A., Payaslı, M., Oztarhan, K., Ozbek, A.S., and Kavuncuoglu, S. (2012). Transient leukemia associated pericardial tamponade in a neonate with Down syndrome. Pediatric hematology and oncology 29: 386-388.

Cakar, N., Kavuncuoglu, S., Aldemir, E., Cetinkaya, M., Guzeltas, A., and Arslan, G. (2014). The features of multiple pregnancies obtained by in vitro fertilization or spontaneously. Pediatrics international 56: 735-741.

Cansev, M., Minbay, Z., Goren, B., Yaylagul, E.O., Cetinkaya, M., Koksal, N., and Alkan, T. (2013). Neuroprotective effects of uridine in a rat model of neonatal hypoxic-ischemic encephalopathy. Neuroscience letters 542:65-70.

Cekmez, F., Canpolat, F.E., Cetinkaya, M., Aydınoz, S., Aydemir, G., Karademir, F., Ceylan, O.M., Ipcioglu, O.M., and Sarıcı, S.U. (2011). Diagnostic value of resistin and visfatin in comparison with C-reactive protein, procalcitonin and IL-6 in neonatal sepsis. European cytokine network 22: 113-117.

Cekmez, F., Canpolat, F.E., Cetinkaya, M., Pirgon, O., Aydınoz, S., Ceylan, O.M., Ipcioglu, O.M., and SarICI, S.U. (2012). IGF-1 and visfatin levels in retinopathy of prematurity. Journal of pediatric ophthalmology and strabismus 49: 120-124.

Cekmez, F., Canpolat, F.E., Erdinc, K., Cetinkaya, M., Akın, O., Pamuk, U., and Sarıcı S.U. (2011). Response to hepatitis B vaccine differs birthweight among neonates. Vaccine 29: 3096-3097.

Cekmez, F., Canpolat, F.E., Pirgon, O., Cetinkaya, M., Aydınoz, S., Suleymanoglu, S., Ipcioglu, O.M., and Sarıcı, S.U. (2011). Apelin, vaspin, visfatin and adiponectin in large for gestational age infants with insulin resistance. Cytokine 56: 387-391.

Cekmez, F., Cetinkaya, M., Tayman, C., Canpolat, F.E., Kafa, I.M., Uysal, S., Tunc, T., and Sarıcı, S.U. (2013). Evaluation of Melatonin and Prostaglandin E1 combination on necrotizing enterecolitis model in neonatal rats. Regulatory peptides 184: 121-125.

Cekmez, F., Purtuloglu, T., Aydemir, G., Aydinoz, S., Fidancı, K., Karaoglu, A., Babacan, O., Canpolat, F.E., Cetinkaya, M., Tunc, T., and Suleymanoglu, S. (2012). Comparing beneficial effects of inhaled nitric oxide to L-arginine in necrotizing enterocolitis model in neonatal rats. Pediatric surgery international 28: 1219-1224. 
Cekmez, F., Tayman, C., Saglam, C., Cetinkaya, M., Bedir, O., Gunal, A., Tunc, T., and Sarıcı S.U. (2012). Well-known but rare pathogen in neonates: Listeria monocytogenes. European review for medical and pharmacological sciences 16 Suppl 4: 58-61.

Celebi, S., Hacımustafaoglu, M., Koksal, N., Ozkan, H., and Cetinkaya, M. (2010). Colistimethate sodium therapy for multidrug-resistant isolates in pediatric patients. Pediatrics international 52: 410-414.

Celebi, S., Hacımustafaoglu, M., Koksal, N., Ozkan, H., Cetinkaya, M., and Ener, B. (2012). Neonatal candidiasis: results of a 8-year old study. Pediatrics international 54: 341349.

Celik, H.G., Celik, E., Yıldırım, G., and Cetinkaya, M. (2017). Do triple test results predict risk of neonatal hyperbilirubinemia? Pakistan journal of medical sciences 33: 979-983.

Celik, H.G., Semerci, S.Y., Yıldırım, G., and Cetinkaya M. (2017). Ininencephaly: a rare congenital anomaly reaching the term. Case reports in perinatal medicine $11 \mathrm{Apr}$. doi: 10.1515/crpm-2016-0056.

Cetinkaya, M., Alkan, T., Ozyener, F., Kafa, I.M., Kurt, M.A., and Koksal, N. (2010). Possible neuroprotective effects of magnesium sulfate and melatonin as both pre- and posttreatment in a neonatal hypoxic-ischemic rat model. Neonatology 99: 302-310.

Cetinkaya, M., Atasay, B., and Perk, Y. (2018). Turkish Neonatal Society guideline on the transfusion principles in newborn. Turk pediatri arsivi 53(Suppl 1): S101-S108.

Cetinkaya, M., Bostan, O., Koksal, N., Semizel, E., Ozkan, H., and Çakır, S. (2011). Early left ventricular diastolic dysfunction in premature infants born to preeclamptic mothers. Journal of perinatal medicine 39: 89-95.

Cetinkaya, M., Buyukkale, G., Payaslı, M., Ozbek, A.S., and Kavuncuoglu, S. (2013). An unusual cause of bilateral scrotal abscess in a preterm infant: Candida Albicans. Brazilian journal of infectious diseases 17: 260-262.

Cetinkaya, M., Cansev, M., Cekmez, F., Tayman, C., Canpolat, F.E., Kafa, I.M., Uysal, S., Tunc, T., and SarıCı, S.U. (2013). CDP-Choline reduces severity of intestinal injury in a neonatal rat model of necrotizing enterocolitis. Journal of surgical research 183:119128.

Cetinkaya, M., Cansev, M., Cekmez, F., Tayman, C., Canpolat, F.E., Kafa, I.M., Yaylagul, E.O., Kramer, B.W., and Sarici S.U. (2015). Protective effects of valproic acid, a histone deacetylase inhibitor, against hyperoxic lung injury in a neonatal rat model. PLoS One 10(5): e0126028.

Cetinkaya, M., Cansev, M., Kafa, I.M., Tayman, C., Cekmez, F., Canpolat, F.E., Tunc, T., and Sarıcı, S.U. (2013). Cytidine 5'-Diphosphocholine (CDP-Choline) ameliorates hyperoxic lung injury in a neonatal rat model. Pediatric research 74: 26-33. 
Cetinkaya, M., Cekmez, F., Buyukkale, G., Erener-Ercan, T., Demir, F., Tunc, T., Aydın, F.N., and Aydemir, G. (2015). Lower vitamin D levels are associated with increased risk of early onset neonatal sepsis in term infants. Journal of perinatology 35: 39-45.

Cetinkaya, M., Cekmez, F., Erener-Ercan, T., Buyukkale, G., Demirhan, A., Aydemir, G., and Aydin, F.N. (2015). Maternal/neonatal vitamin D deficiency: a risk factor for bronchopulmonary dysplasia in preterms? Journal of perinatology 35: 813-817.

Cetinkaya, M., Cekmez, F., Canpolat, F.E., Akın, O., Atabek, C., Kozan, S., and Sarıcı S.U. (2012). Osephageal atresia in a neonate with translocation $t(1 ; 13)$. Genetic counseling 23: 73-75.

Cetinkaya, M., Durmaz, O., Buyukkale, G., Ozbek, S., Acar, D., Kilıcaslan, I., and Kavuncuoglu S. (2013). Neonatal Bartter Syndrome and unilateral ectopic cyst as new renal causes of hydrops fetalis: two case reports and review of the literature. The journal of maternal-fetal \& neonatal medicine 26: 1147-1150.

Cetinkaya, M., Ercan, T.E., Saglam, O.K., Buyukkale, G., Kavuncuoglu, S., and Mete, F. (2014). Efficacy of prophylactic fluconazole therapy in decreasing the incidence of Candida infections in extremely low birth weight infants. American journal of perinatology 31: 1043-1048.

Cetinkaya, M., Erener-Ercan, T., Cansev, M., Buyukkale, G., Cekmez, F., Aydemir, G., Pirhan, D., Genc, F.A., Acar, E., and Taskin, N. (2014). The utility of serial plasma sEselectin measurements in the prediction of retinopathy of prematurity in premature infants. Early human development 90: 517-521.

Cetinkaya, M., Erener-Ercan, T., Kalayci-Oral, T., Babayigit, A., Cebeci, B., Semerci, S.Y., and Buyukkale, G. (2017). Maternal/neonatal vitamin D deficiency: a new risk factor for necrotizing enterocolitis in preterm infants? Journal of perinatology 37: 673-678.

Cetinkaya, M., Koksal, N., Ozkan, H., Donmez, O., Saglam, H., and Kırıstıoglu, I. (2008). Hyperprostaglandin $\mathrm{E}$ syndrome: use of indomethacin and steroid, death due to necrotizing enterocolitis and sepsis. Turkish journal of pediatrics 50: 386-390.

Cetinkaya, M., Oral, T.K., Karatekin, S., Cebeci, B., Babayigit, A., and Yesil, Y. (2017). Efficacy of palivizumab prophylaxis on the frequency of RSV-associated lower respiratory tract infections in preterm infants: determination of the ideal target population for prophylaxis. European journal of clinical microbiology \& infectious diseases 36: 1629-1634.

Cetinkaya, M., Ozen, S., Uslu, S., Gonc, N., Acunas, B., Akıncı, A., Satar, M., and Berberoglu, M. (2018). Diagnostic and therapeutic approach in newborns with ambiguous genitale with disorder of sex development: consensus report of Turkish Neonatal and Pediatric Endocrinology and Diabetes Societies. Turk pediatri arsivi 53 (Suppl 1): S198-S208. 
Cetinkaya, M., Ozkan, H., Celebi, S., Koksal, N., and Hacımustafaoglu, M. (2011). Human 2009 influenza A (H1N1) virus infection in a premature infant born to a H1N1-infected mother: placental transmission? Turkish journal of pediatrics 53: 441-444.

Cetinkaya, M., Ozkan, H., and Koksal N. (2008). Unilateral radius aplasia due to lamotrigine and oxcarbazepine use in pregnancy. The journal of maternal-fetal \& neonatal medicine 21: 927-930.

Cetinkaya, M., Ozkan, H., and Koksal, N. (2012). A new scoring system for evaluation of multiple organ dysfunction syndrome in premature infants: modified NEOMOD scoring system. American journal of critical care 21: 328-337.

Cetinkaya, M., Ozkan, H., and Koksal, N. (2012). Maternal preeclampsia is associated with increased risk of necrotizing enterocolitis in preterm infants. Early human development 88: 893-898.

Cetinkaya, M., Ozkan, H., Koksal, N., Akacı, O., and Ozgur, T. (2010). The efficacy of serial serum amyloid A measurements for diagnosis and follow-up of necrotizing enterocolitis in premature infants. Pediatric surgery international 26: 835-841.

Cetinkaya, M., Ozkan, H., Koksal, N., Akacı, O., and Ozgur, T. (2011). Comparison of the efficacy of serial serum amyloid A, C-reactive protein and procalcitonin in the diagnosis and follow-up of necrotizing enterocolitis in premature infants. Journal of pediatric surgery 46: 1482-1489.

Cetinkaya, M., Ozkan, H., Koksal, N., Celebi, S., and Hacımustafaoglu, M. (2009). Comparison of serum amyloid A concentrations with those of C-reactive protein and procalcitonin in diagnosis and follow-up of neonatal sepsis in premature infants. Journal of perinatology 29: 225-231.

Cetinkaya, M., Ozkan, H., Koksal, N., Coskun, S.Z., Hacımustafaoglu, M., and Girisgin, O. (2008). Neonatal myiasis: a case report. Turkish journal of pediatrics 50: 581-584.

Cetinkaya, M., Ozkan, H., Koksal, N., Karalı, Z., and Ozgur T. (2010). Neonatal outcomes of premature infants born to preeclamptic mothers. The journal of maternal-fetal \& neonatal medicine 23: 425-430.

Cetinkaya, M., Ozkan, H., Koksal, N., Sarısozen, B., and Yazıcı, Z. (2012). Osteofibrous dysplasia associated with pathological tibia fracture: a case report and review of the literature. Journal of pediatric orthopedics. Part B. 21: 183-186.

Cetinkaya, M., Ozkan, H., Koksal, N., Yazıcı, Z., and Yalcınkaya U. (2008). Spondylocostal dysostosis associated with diaphragmatic hernia and neural tube defects. Clinical dysmorphology 17: 151-154. 
Cetinkaya, M., Semizel, E., Bostan, O.M., and Cil, E. (2008). Risk of vasovagal syncope and cardiac arrhythmias in children with mitral valve prolapse. Acta cardiologica 63: 395398.

Donmez, O., Cetinkaya, M., and Canbek, R. (2005). Hemoperfusion in a child with amitriptyline intoxication. Pediatric nephrology 20: 105-107.

Erdeve, O., Okulu, E., Olukman, O., Ulubas, D., Buyukkale, G., Narter, F., Tunc, G., Atasay, B., Gultekin, N.D., Arsan, S., Koc. E., and Turkish Neonatal Jaundice Registry Collabolators. (2018). The Turkish Neonatal Jaundice Online Registry: A National Root Cause Analysis. PLoS One Feb 23;13(2): e0193108.

Erdeve, O., Okulu, E., Tunc, G., Celik, Y., Kayacan, U., Cetinkaya, M., Buyukkale, G., Ozkan, H., Koksal, N., Satar, M., Akcali, M., Aygun, C., Ozkıraz, S., Zubarioglu, U., Unal, S., Turgut, H., Mert, K., Gokmen, T., Akcan, B., Atasay, B., Arsan, S., and Rescue HFOV Trial Group. (2019). An observational, prospective, multicenter study on rescue highfrequency oscillatory ventilation in neonates failing with conventional ventilation. PLoS One June 10; 14(6): e0217768.

Guven, S., Bozdag, S., Saner, H., Cetinkaya, M., Yazar, A.S., and Erguven, M. (2013). Early neonatal outcomes of volume guaranteed ventilation in preterm infants with respiratory distress syndrome. The journal of maternal-fetal \& neonatal medicine 26: 396-401.

Kalaycı, T., Ercan, T.E., Buyukkale, G., and Cetinkaya, M. (2015). Limb deformity in a newborn. Is rifampicin just an innocent bystander? European review for medical and pharmacological sciences 19: 517-519.

Kaya, G., Saldir, M., Polat, A., Fidanci, M.K., Erdem, A., Erdem, G., Kurt, Y.G., Cetinkaya, M., Cekmez, F., Onguru, O., and Tunc, T. (2016). Evaluation of etanercept treatment in newborn rat model with hyperoxic lung injury. Fetal and pediatric pathology 35 (5): 327-338.

Koksal, N., Akpınar, R., and Cetinkaya, M. (2009). Early Administration of the second surfactant dose in severe respiratory distress syndrome. Turkish journal of pediatrics 51: 556-564.

Koksal, N., Calıskan, B., Saglam, H., Yazıcı, Z., and Cetinkaya M. (2008). Reference values for neonatal thyroid volumes in a moderately iodine deficient area. Journal of endocrinological investigation 31: 642-646.

Koksal, N., Harmancı, R., Cetinkaya, M., and Hacımustafaoglu, M. (2007). Role of procalcitonin and CRP in diagnosis and follow-up of neonatal sepsis. Turkish journal of pediatrics 49:21-29. 
Koksal, N., Kavurt, A.V., Cetinkaya, M., Ozarda, Y., and Ozkan, H. (2011). Comparison of lipid emulsions on antioxidant capacity in preterms receiving parenteral nutrition. Pediatrics international 53: 562-566.

Koksal, N., Kayık, B., Cetinkaya, M., Ozkan, H., Budak, F., Kılıc, S., Canıtez, Y., and Oral, B. (2012). Value of serum and bronchoalveolar fluid lavage pro- and anti-inflammatory cytokine levels in predicting bronchopulmonary dysplasia in premature infants. European cytokine network 23: 29-35.

Koyuncuoglu, T., Turkyılmaz, M., Goren, B., Cetinkaya, M., Cansev, M., and Alkan, T. (2015). Uridine protects against hypoxic-ischemic brain injury by reducing histone deacetylase activity in neonatal rats. Restorative neurology and neuroscience 33: 777784.

Kurnaz, D., Semerci, S.Y., Babayigit, A., Cebeci, B., Buyukkale, G., and Cetinkaya, M. (2018). Angioedema associated with nebulized recombinant human DNase in a preterm infant: case report. Journal of pediatric pharmacology and therapeutics 23: 219-222.

Ozkan, H., Cetinkaya, M., and Koksal, N. (2012). Increased incidence of bronchopulmonary dysplasia in preterm infants exposed to preeclampsia. The journal of maternal-fetal \& neonatal medicine 25: 2681-2685.

Ozkan, H., Cetinkaya, M., Koksal, N., Ali, R., Gunes, A.M., Baytan, B., Ozkalemkas, F., Ozkocaman, V., Ozcelik, T., Gunay, U., Tunalı, A., Kimya, Y., and Cengiz, C. (2010). Neonatal outcomes of pregnancy complicated by idiopathic thrombocytopenic purpura. Journal of perinatology 30: 38-44.

Ozkan, H., Cetinkaya, M., Koksal, N., Celebi, S., and Hacımustafaoglu, M. (2009). Pseudomonas aeroginasa pleural empyema in a preterm infant. Turkish journal of pediatrics 51: 395-398.

Ozkan, H., Cetinkaya, M., Koksal, N., Celebi, S., and Hacımustafaoglu, M. (2014). Cultureproven neonatal sepsis in preterm infants in a neonatal intensive care unit over a 7year period: Coagulase-negative Staphylococcus as the predominant pathogen. Pediatrics international 56: 60-66.

Ozkan, H., Cetinkaya, M., Koksal, N., Ozmen, A., and Yıldız, M. (2011). Maternal preeclampsia is associated with an increased risk of retinopathy of prematurity. Journal of perinatal medicine 39: 523-527.

Ozkan, H., Cetinkaya, M., Koksal, N., and Yapıcı, S. (2011). Severe fetal valproate syndrome: combination of complex cardiac defect, multicystic dysplastic kidney, and trigonocephaly. The journal of maternal-fetal \& neonatal medicine 24: 521-524. 
Ozkan, H., Koksal, N., Cetinkaya, M., Kılıc, S., Celebi, S., Oral, B., and Budak, F. (2012). Serum mannan-binding lectin (MBL) gene polymorphism and low $\mathrm{MBL}$ levels are associated with neonatal sepsis and pneumonia. Journal of perinatology 32: 210-217.

Ozyener, F., Cetinkaya, M., Alkan, T., Goren, B., Kafa, I.M., Kurt, M.A., and Koksal N. (2012). Neuroprotective effects of melatonin administered alone or in combination with topiramate in neonatal hypoxic-ischemic rat model. Restorative neurology and neuroscience 30: 435-444.

Sahiner F, Cekmez F, Cetinkaya M, Kaya G, Kalaycı T, Gunes O, Sener K, Yapar M, Tunc T, Ecemis T, Cekmez Y, Kubar A. (2015) Congenital cytomegalovirus infections and glycoprotein genotypes in live-born infants: a prevalence study in Turkey. Infectious diseases (London, England) 47: 465-471.

Saldir, M., Tunc, T., Cekmez, F., Cetinkaya, M., Kalayci, T., Fidanci, K., Babacan, O., Erdem, G., Kocak, N., Sari, E., Akgul, E.O., and Kul, M. (2015). Endocan and soluble triggering receptor expressed on myeloid cells as novel markers for neonatal sepsis. Pediatrics and neonatology 56: 415-421.

Semerci, S.Y., Babayigit, A., Cebeci, B., Buyukkale, G., and Cetinkaya, M. (2016). Hepatic abscesses in preterm infants: report of three cases and review of the literature. Journal of tropical pediatrics 62: 255-260.

Semerci, S.Y., Babayigit, A., Cebeci, B., Buyukkale, G., and Cetinkaya M. (2017) Cytomegalovirus as a seldom cause of non-immune hydrops fetalis: case report and review of the literature. Iranian red crescent medical journal 19(8): e55570.

Semerci, S.Y., Bornaun, H., Kurnaz, D., Cebeci, B., Babayigit, A., Buyukkale, G., and Cetinkaya, M. (2018). Neonatal atrial flutter: Three cases and review of the literature. Turkish journal of pediatrics 60: 306-309.

Semerci, S.Y., Kurnaz, D., Babayigit, A., Cebeci, B., Buyukkale, G., Cetinkaya, M., Guvenc, U., and Kucur, O. (2017). Neonatal appendicitis as a rare cause of intestinal perforation in a preterm neonate. Journal of the college of physicians and surgeans pakistan 27: S38-S40.

Semerci, S.Y., Yucel, B., Erbas, I.M., Gunkaya, O.S., Talmac, M., and Cetinkaya, M. (2018). The utility of amniotic fluid $\mathrm{pH}$ and electrolytes for prediction of neonatal respiratory morbidities. Journal of maternal-fetal \& neonatal medicine Jul 22: 1-5.

Suleymanoglu, S., Cekmez, F., Cetinkaya, M., Tayman, C., Canpolat, F.E., Kafa, I.M., Tanju, A., Arzıman, I.K., Fidancı, M., Tunc, T., and Sarıcı, S.U. (2014). Protective effects of melatonin therapy in model for neonatal hyperoxic lung injury. Alternative therapies in health and medicine 20: 24-29. 
Tayman, C., Cekmez, F., Kafa, I.M., Canpolat, F.E., Cetinkaya, M., Uysal, S., Tunc, T., and Sarıcı S.U. (2012). Beneficial effects of Nigella sativa oil on intestinal damage in necrotizing enterocolitis. Journal of investigative surgery 2012; 25: 286-294.

Tayman, C., Cekmez, F., Kafa, I.M., Canpolat, F.E., Cetinkaya, M., Uysal, S., Tunc, T., and Sarıcı, S.U. (2013). Protective effects of Nigella sativa oil on hyperoxia-induced lung injury. Archivos de bronchoneumologia 49: 15-21.

Tunc, T., Cekmez, F., Cetinkaya, M., Kalayci, T., Fidanci, K., Saldir, M., Babacan, O., Sari, E., Erdem, G., Cayci, T., Kul, M., and Kavuncuoglu S. (2015). Diagnostic value of elevated CXCR4 and CXCL12 in neonatal sepsis. Journal of maternal-fetal \& neonatal medicine 28: 356-361.

Tuzun, F., Ozkan, H., Cetinkaya, M., Yucesoy, E., Kurum, O., Cebeci, B., Cakmak, E., Ozkutuk, A., Keskinoglu, P., Baysal, B., Kumral, A., and Duman, N. (2019). Is European Medicines Agency (EMA) sepsis criteria accurate for neonatal sepsis diagnosis or do we need new criteria? PLoS One Jun 6;14(6): e021800. 


\section{Acknowledgement}

It is a great honour for me to complete my PhD-thesis at Maastricht University, The Netherlands. I hope to continue my research line on the improvement of neonatal health in collaboration with Maastricht University.

I owe my special thanks to my supervisor at Maastricht University, Prof. B. W. Kramer, for giving me the opportunity to pursue a PhD at Maastricht University. Because he always encouraged and supported me, I thank him for this kind support from past to future.

I also want to thank my second supervisor, Professor T. Delhaas, for his valuable comments during the writing and editing stages of my thesis.

A word of thank to my co-supervisor Dr. D. Gavilanes and to the members of the Assessment Committee.

I also want to thank my colleagues Prof. M. Cansev, Prof. T. Alkan, Assoc. Prof. Dr. I.M. Kafa and all others from Uludag University, Faculty of Medicine, Departments of Physiology, Pharmacology and Anatomy for their collaboration during the experimental studies. I also want to thank to Prof. N. Koksal, Prof. E. Koc and Prof. E. Ozek for supporting me in all scientific activities.

Lastly, I want to thank to my mother, sisters, dear friends who all supported me during all my scientific improvement stages. 

